\title{
MRI-based Measurement of Structural and Functional Connectivity in Health and Disease
}

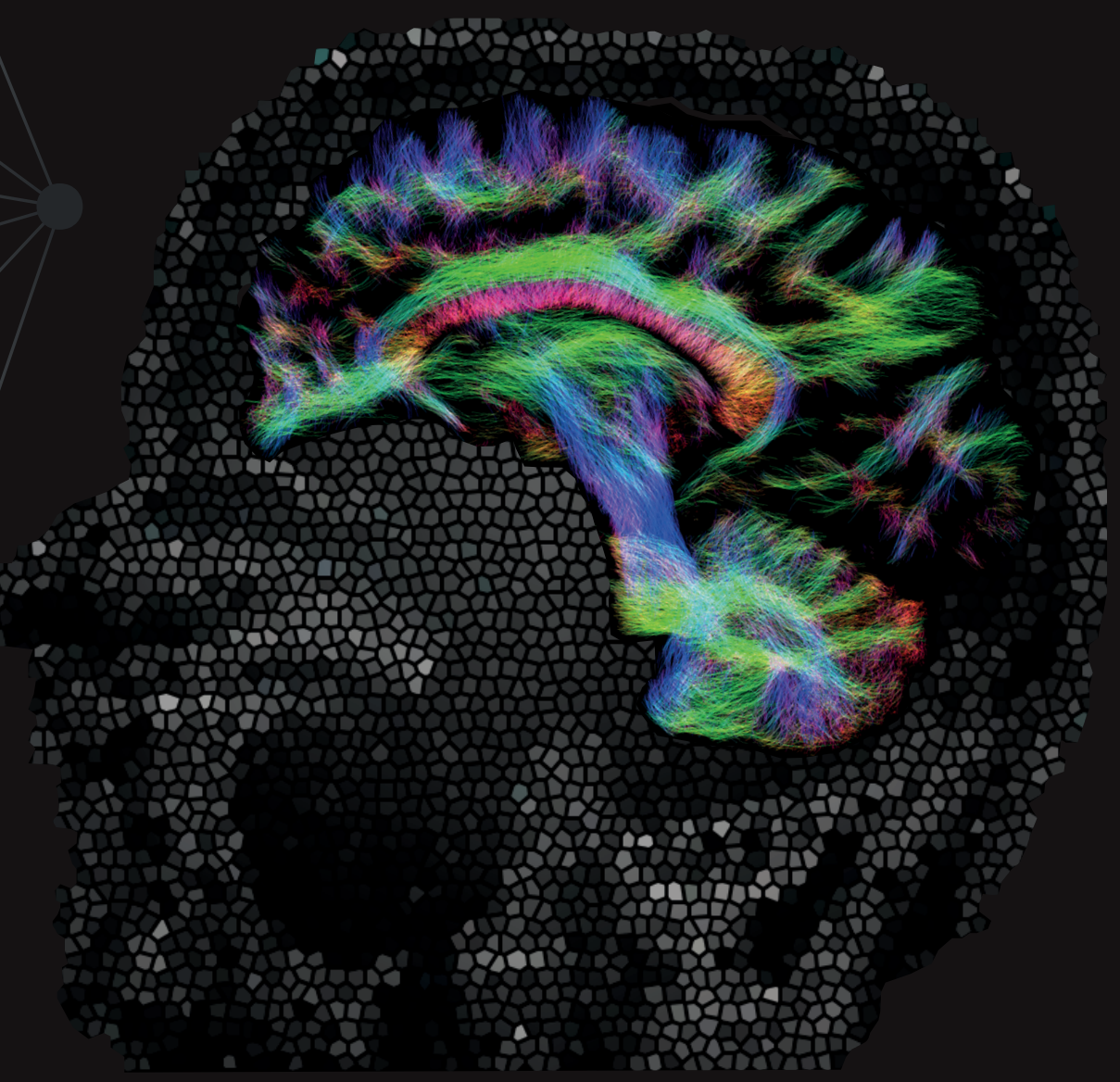





\section{MRI-based Measurement of Structural and Functional Connectivity in Health and Disease}

Michel Rogier Theodoor Sinke 


\section{Reading Committee}

prof. dr. H.E. Hulshoff Pol

prof. dr. J.P.H. Burbach

prof. dr. G.J. Biessels

prof. dr. C.J. Stam

dr. ir. M. Froeling

\section{Paranymphs}

Bart Molenaar

Jos Evers

\section{Design}

Michel R.T. Sinke

\section{Printing \\ ProefschriftMaken || www.proefschriftmaken.nl}

ISBN: 978-94-6380-817-0

Copyright (C) Michel R.T. Sinke, 2020

All rights reserved. No part of this thesis may be reproduced, stored in a retrieval system, or transmitted in any form or by any means without prior permission of the holder of the copyright. 


\title{
MRI-based Measurement of Structural and Functional Connectivity in Health and Disease
}

\author{
MRI-metingen van structurele en functionele \\ connectiviteit in gezondheid en ziekte \\ (met een samenvatting in het Nederlands)
}

\section{Proefschrift}

ter verkrijging van de graad van doctor aan de

Universiteit Utrecht

$$
\text { op gezag van de }
$$

rector magnificus, prof.dr. H.R.B.M. Kummeling, ingevolge het besluit van het college voor promoties in het openbaar te verdedigen op

maandag 6 juli 2020 des middags te 12.45 uur

door

\section{Michel Rogier Theodoor Sinke}

geboren op 27 oktober 1986

te Gouda 


\section{Promotor:}

Prof. dr. R.M. Dijkhuizen

\section{Copromotor:}

Dr. W.M. Otte

Dit proefschrift werd (mede) mogelijk gemaakt met financiële steun van de Nederlandse Organisatie voor Wetenschappelijk Onderzoek (NWO-VICI 016.130.662, NWO-VENI 016.168.038), de Hersenstichting [F2014(1)-06] en Qatar National Research Fund (grant nummers NPRP-5-381-3-101 en NPRP-7-1648-3420). 
Alleen

Samen 



\section{Contents}

\section{Chapter 1}

General Introduction, Aims and Outline

\section{Chapter 2}

Bayesian Exponential Random Graph Modelling of Whole-Brain Structural Networks across Lifespan

\section{Chapter 3}

Diffusion MRI-Based Cortical Connectome Reconstruction - Dependency on Tractography Procedures and Neuroanatomical Characteristics

\section{Chapter 4}

Modified Structural Network Backbone in the Contralesional Hemisphere Chronically after Stroke in Rat Brain

\section{Chapter 5}

Differences in Structural and Functional Networks between Young Adult and Aged Rat Brains before and after Stroke Lesion Simulations

\section{Chapter 6}

Remote Corticospinal Tract Degeneration after Cortical Stroke in Rats May not Preclude Spontaneous Sensorimotor Recovery

\section{Chapter 7}

Imaging Markers for the Characterization of Gray and White Matter Changes from Acute to Chronic Stages after Experimental Traumatic Brain Injury

\section{Chapter 8}

General Discussion

Supplementary Figures

\section{References}

\section{Addenda}

Summary

Samenvatting

Dankwoord 


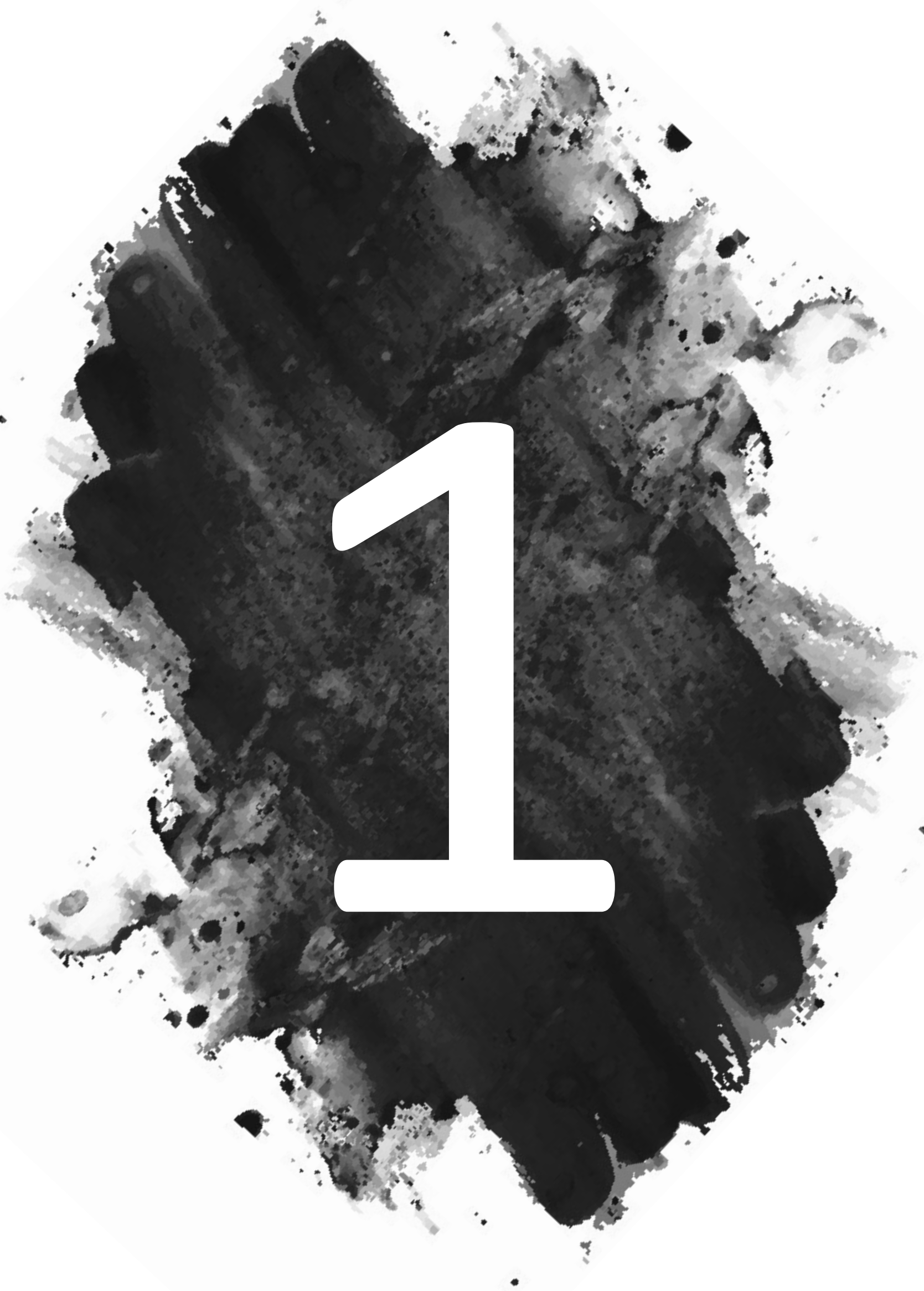




\section{Chapter 1}

General Introduction,

Aims and Outline 



\section{GENERAL INTRODUCTION}

\section{Introduction}

This chapter introduces the most important topics and concepts of the present thesis. First, the network nature of the mammalian brain, the consequences of brain damage and the concept of brain plasticity are briefly introduced. Next, an overview of different forms of brain connectivity is given, followed by an introduction of magnetic resonance imaging (MRI) techniques as non-invasive tools to map brain connectivity. Subsequently, brain network science is introduced, including its application for the understanding of brain reorganization in relation to ageing and brain damage. Next, animal models that may bridge the gaps in our understanding of patterns in brain reorganization after acute brain injury, specifically stroke and traumatic brain injury (TBI), are introduced. Lastly, this chapter concludes with the main aims and outline of this thesis.

\section{The mammalian brain - a complex integrative network}

The mammalian brain consists of grey matter and white matter. The grey matter is mainly constituted by neuronal cell bodies, whereas the white matter contains long myelinated axons and larger fibre bundles. The axons and fibre bundles form the brain's wiring that interconnects different brain regions, and also connects brain regions to other organs and limbs via the spinal cord. This allows for information transfer from the body and sensory organs to the brain, as well as from the brain to the body, in order to respond and adapt to the environment. Furthermore, it allows for information processing and integration within the brain by interaction between different functional regions. As such, the mammalian brain can be regarded as a highly complex and integrative network, consisting of spatially distributed regions that continuously interact with each other to enable mammalian brain function and behaviour (Bassett and Sporns, 2017; Bassett et al., 2018; Bullmore and Sporns, 2009; van den Heuvel and Hulshoff Pol, 2010). As a consequence, it can be considered critical to study consequences of brain disorders from a network perspective to optimally understand pathological processes and recovery mechanisms (Bassett and Bullmore, 2009; Grefkes and Fink, 2014; Stam, 2014).

\section{Brain damage and plasticity}

Every year millions of people worldwide suffer from brain damage due to tumours, brain trauma, stroke, neurodegeneration or secondarily from other diseases. Brain damage may be focal or global. However, due to the network nature of the brain, a focal lesion in a specific functional region or white matter tract could still have global consequences, as connections and interactions with distant functional regions may be disturbed - a phenomenon called diaschisis (Carrera and Tononi, 2014). Loss of neuronal function due to brain damage can lead to behavioural and cognitive impairment. Nevertheless, most 
patients show a certain degree of spontaneous recovery in the following weeks and months after acute brain injuries, such as TBI and stroke. This spontaneous recovery has been related to brain plasticity mechanisms. Brain plasticity refers to the brain's capability to compensate for lost brain function by reorganization of neuronal networks. However, the mechanisms underlying plasticity and how these relate to functional recovery remains largely unknown. Improved knowledge on the underlying mechanisms related to brain damage and plasticity may ultimately lead to better diagnosis, prognosis and therapeutic strategies after acute brain injuries like stroke and TBI.

\section{Brain connectivity - structural, functional and effective connectivity}

Brain connectivity refers to the overall pattern of anatomical links and functional interactions between different regions or elements in the brain. It ranges from micro- to macro-scale and from single neuronal connections to whole-brain networks. Brain connectivity can be divided into two main categories: (I) structural connectivity and (II) functional connectivity. A third form, i.e. effective connectivity, has been defined as a variant of functional connectivity (Friston, 2011).

Structural connectivity refers to the anatomical connections in the brain, i.e. the axonal connections at the micro-scale as well as larger white matter fibre bundles at the meso- and macro-scale. Anatomical connections at the micro- and meso-scale can be mapped with histology and neuronal tracers (Heimer and Robards, 1981). In addition, some neuronal tracers enable the determination of the directionality of these structural connections. Due to its invasive nature, histology and neuronal tracers can only be applied in animal studies or on human post-mortem tissue. Today, diffusion-weighted magnetic resonance imaging offers the only non-invasive tool to map structural connections at the meso- and macroscale in vivo and post-mortem in animals and humans (Calabrese et al., 2015; Johansen-Berg and Rushworth, 2009; Jones, 2008).

Functional connectivity refers to the statistical dependence or correlation between 'spontaneous' activity patterns from different regions. Effective connectivity is a specific form of functional connectivity, by which causal or directional relationships are explicitly modelled. The concepts of functional and effective connectivity can be applied to various forms of brain recordings across different scales. First, to imaging techniques that assess neural activity more directly, such as electrophysiology at the micro-scale, electrocorticography (Ortega et al., 2008) and electroencephalography (van Diessen et al., 2015) at the meso- and macro-scale, as well as optical imaging techniques across multiple scales (Lim et al., 2014). Second, to imaging techniques that assess neural activity more indirectly such as blood oxygenation level-dependent (BOLD) resting-state functional MRI (Fox and Raichle, 2007), optical intrinsic signal imaging (Bauer et al., 2014, 2017; White et 
al., 2011) and magnetoencephalography (van Diessen et al., 2015) at the meso- and macroscale.

\section{MRI - a non-invasive technique to map structural and functional brain connectivity}

MRI can be used to map structural and functional brain connectivity. MRI is a non-invasive brain imaging technique that can be roughly divided into structural MRI and functional MRI methods. Structural MRI provides information on the anatomy of the brain, whereas functional MRI can provide dynamic physiological information (Friston, 2011). Recent developments in structural and functional MRI have made it possible to measure structural and functional brain connectivity non-invasively over time in humans and animals. Hence, MRI techniques offer a unique translational way to study structural and function brain organization in health and disease (Bassett and Bullmore, 2009; Dijkhuizen and Nicolay, 2003; Dijkhuizen et al., 2012; Hoyer et al., 2014).

\section{Structural MRI}

Structural MRI can be used to make an anatomical image of the brain and to study its anatomical properties (Dijkhuizen and Nicolay, 2003; Friston, 2011). Moreover, anatomical images can be used to align brain images of different subjects to each other, or to a reference template containing pre-defined functional atlas regions, to enable betweensubject or within-subject comparisons.

Structural MRI processing methods and analysis tools make use of the imaging contrast between different tissues, such as grey matter, white matter, cerebral spinal fluid or pathological tissue, to measure specific anatomical (e.g. cortical thickness) or pathological properties (e.g. stroke lesion volume). For example, $\mathrm{T}_{2}$-weighted and diffusion-weighted $\mathrm{MRI}$ are established clinical tools for detection of stroke lesions. Acute post-stroke cytotoxic oedema (i.e. cell swelling) leads to reduction in water diffusion, whereas vasogenic oedema and chronic tissue degeneration lead to an increase in water diffusion and prolonged $\mathrm{T}_{2}$ (transverse MR relaxation time) (Dijkhuizen and Nicolay, 2003). Therefore diffusionweighted and $\mathrm{T}_{2}$-weighted imaging are useful tools to detect oedema formation or fluid accumulation, and to segment lesioned brain tissue.

\section{Diffusion MRI-based tractography and structural connectivity}

Diffusion tensor imaging (DTI) is specifically useful for the characterization of white matter tissue and tracts, which informs on structural connectivity (Basser et al., 1994a; Le Bihan et al., 1986; Jbabdi and Johansen-Berg, 2011; Jones, 2008; Mori et al., 1999). DTI makes use of the random motion (i.e. Brownian motion) of water molecules in tissue. When there is no restriction or hindrance the diffusion of water molecules is equal in all directions, which is called isotropic diffusion. However, in the brain water diffusion is restricted and hindered 
by lipid structures like cell membranes and myelin of the white matter. Therefore, the diffusion of water molecules is asymmetric across different directions, which is called anisotropic diffusion. For example, there is more diffusional displacement along than perpendicular to the axonal fibre bundles. DTI uses this principle to determine the diffusivity (i.e. eigenvalues) along three different directions (i.e. eigenvectors). Based on the diffusivity values, several diffusion metrics can be calculated. The axial diffusivity (AD), which is the diffusivity along axonal fibre bundles, the radial diffusivity (RD), the diffusivity perpendicular to axonal fibre bundles, the mean diffusivity (MD) and the fractional anisotropy (FA). These metrics can be used to characterize white matter structure and to detect white matter alterations, for example related to ageing or brain pathology. For instance, RD may decrease after demyelination or MD may increase after oedema formation or fluid accumulation (Calabrese and Johnson, 2013; Mori and Zhang, 2006; Tu et al., 2016; Winklewski, 2018).

DTI can also be used to map white matter tracts by a technique called fibre-tractography (Basser et al., 2000; Jbabdi and Johansen-Berg, 2011; Jbabdi et al., 2015; Jeurissen et al., 2017). The diffusion tensor informs on the main direction (i.e. principal eigenvector) of the axonal fibre bundles in each voxel. Consequently, the fibre bundles can be reconstructed by a step-wise approach where streamlines are propagating voxel-wise from one region to another region, until they reach a voxel that violates certain criteria, i.e. in grey matter, cerebrospinal fluid or locations with unrealistically strong pathway bending. With this approach, structural connections can be mapped between specific regions or at a wholebrain level. In addition, the $A D, R D, M D$ and FA across specific white matter tracts can be determined.

Although DTI is a promising and informative technique, it is insensitive to complex fibre orientations within voxels, such as bending and crossing fibers. These complex fibre orientations have been shown to occur in about $90 \%$ of white matter voxels in the human brain (Jeurissen et al., 2013). Therefore, so-called higher order models have been developed, such as constrained spherical deconvolution (CSD) (Jeurissen et al., 2014; Tournier et al., 2011, 2007), which allow the modelling of more complex fibre orientations. These higher order models and related tractography approaches, such as CSD-based tractography and global tractography, may yield more reliable reconstructions of white matter tracts and whole-brain structural networks, and may better inform on structural network alterations in health and disease (Christiaens et al., 2015a; Tournier et al., 2007, 2012).

\section{Functional MRI}

Functional MRI ( $\mathrm{fMRI}$ ) has been widely used to study brain function in relation to execution of behavioural or cognitive tasks in health and disease. The neuronal activation in specific regions is indirectly measured by the blood oxygenation level-dependent (BOLD) signal. 
Task-based fMRI studies that have been applied in brain disorders include investigations of post-injury functional brain reorganization in relation to behavioural outcome, for example after stroke (van Meer et al., 2012; Rehme and Grefkes, 2013; Rehme et al., 2012).

\section{Resting-state fMRI and functional connectivity}

Resting-state $\mathrm{fMRI}$ is a popular application of $\mathrm{fMRI}$, performed during 'resting state', so without task or sensory stimulation during data acquisition, which can be used to map functional connectivity in the brain. Resting-state $\mathrm{FMRI}$ indirectly measures the intrinsic 'spontaneous' neural activity in the brain from BOLD signals. Low-frequency BOLD fluctuations, reflecting spontaneous brain activity, are highly coherent in functionally related brain regions, where the degree of synchronization is a measure of functional connectivity (Biswal et al., 1995; Fox and Raichle, 2007). The spontaneous BOLD fluctuations have been shown to be related to neuronal activity in animal electrophysiology studies (Magri et al., 2012; Pan et al., 2011; Shmuel and Leopold, 2008) as well as to slow cortical potentials in humans (He et al., 2008). Hence, resting-state fMRI can be used to map macroscale functional connectivity non-invasively from separate functional connections to wholebrain functional networks. Over the years, several specific resting-state functional networks have been identified, such as the default mode network, in both humans and rats (Gozzi and Schwarz, 2016; Greicius et al., 2003; Lu et al., 2012; Sierakowiak et al., 2015). Changes in specific functional networks as well as in whole-brain functional networks have been related to behavioural functioning in health and disease (Bassett and Bullmore, 2009; Broyd et al., 2009; Dijkhuizen et al., 2014; van den Heuvel and Hulshoff Pol, 2010; van Meer et al., 2010a).

\section{Brain network science - characterizing brain connectivity from a network perspective}

Brain network science involves the study and characterization of brain connectivity from a network perspective. Brain network science describes the mammalian brain as a collection of nodes (i.e. the functional brain regions, such as the primary motor cortices) and edges (i.e. the anatomical or functional connections between those regions). The edges can be either binary or weighted. Binary edges only indicate the presence or absence of specific connections, whereas weighted edges may also indicate the strength or size of those connections. Based on the specific constitution of connections and strengths, various network metrics can be calculated, which provide quantitative information on the topological properties of these networks (Bullmore and Sporns, 2009; van den Heuvel et al., 2012; Rubinov and Sporns, 2010). The healthy brain has been characterized as a complex network that effectively combines global efficiency (i.e. integration) with local specialization (i.e. segregation) (Bullmore and Sporns, 2012). This unique topology with high levels of integration and segregation is characterized by a small-world organization, 
modularity and a 'rich club' of highly connected hub regions (Bassett and Bullmore, 2009; Bassett and Sporns, 2017; Bullmore and Sporns, 2009; Sporns, 2010; Watts and Strogatz, 1998). These topological characteristics have been found across species (van den Heuvel et al., 2015, 2016a, 2016b), and deviation from optimal organization has been observed in relation to aging, brain dysfunction and multiple brain disorders, including stroke and brain trauma (Bullmore and Sporns, 2009; Bullmore et al., 2009; van Meer et al., 2012; Sporns, 2010; Stam, 2014).

Over the last decades several network analysis tools have been developed to study brain networks. These tools range from descriptive models, such as classical graph analysis (Bullmore and Sporns, 2009) and minimum spanning tree analysis (Stam et al., 2014), to more complex and generative models like Bayesian exponential random graph models (Simpson et al., 2011), mixed-effect models (Simpson and Laurienti, 2015b) and Gibbs distribution models (La Rosa et al., 2016). Given the complex network nature of the brain, network analysis techniques may offer new - or additional - insights in structural and functional brain reorganization related to brain disorders such as stroke and TBI (Carter et al., 2012a; Grefkes and Fink, 2011; Rehme and Grefkes, 2013). Network analysis tools explicitly take into account the interdependencies between functionally connected regions, thereby providing information on both locational changes as well as global topological alterations. Network analyses tools have been successfully used to characterize brain connectivity in the healthy and diseased brain (Bassett and Bullmore, 2009; Dijkhuizen et al., 2014; Stam, 2014) across different species (van den Heuvel et al., 2016a), and across the lifespan (Damoiseaux, 2017; Otte et al., 2015; Sinke et al., 2016).

\section{Brain reorganization related to ischemic stroke}

Ischemic stroke is characterized by a sudden loss of blood flow to a particular part of the brain and is one of the main causes of long-term disability in adults (Feigin et al., 2014). The loss of blood flow in the brain leads to neuronal damage, loss of neuronal function and ultimately to behavioural or sensorimotor impairments (or even death). However, many patients show (partial) recovery in the weeks and months following a stroke. Post-stroke functional recovery has been related to restoration and reorganization of surviving networks in the brain (Cramer, 2008; Grefkes and Fink, 2011; Jiang et al., 2013; Jones, 2017; Murphy and Corbett, 2009). This neural network reorganization occurs from micro-scale (i.e. axonal sprouting, synaptic plasticity) to macro-scale (i.e. cortical remapping) (Biernaskie and Corbett, 2001; Jones et al., 1996; Stroemer et al., 1995), and also from proximal (i.e. periand ipsilesional) to more distant (i.e. contra-lesional) sites (Cai et al., 2016; Crofts et al., 2011; Dacosta-Aguayo et al., 2014; Granziera et al., 2012; Gratton et al., 2013; JohansenBerg et al., 2010; Schaechter et al., 2010). Therefore, assessment of structural and functional whole-brain connectivity may be critical for optimal understanding of functional 
consequences of stroke and post-stroke recovery mechanisms (Carter et al., 2012a; Grefkes and Fink, 2011; Rehme and Grefkes, 2013).

Both clinical and preclinical studies have employed structural and functional MRI to investigate alterations in structural and functional connectivity after stroke (Crofts et al., 2011; Dijkhuizen and Nicolay, 2003; Grefkes and Fink, 2011; Johansen-Berg et al., 2007). Diffusion MRI in stroke patients has revealed decreased white matter integrity (i.e. reduced FA with increased MD) in the bilateral CST (Schaechter et al., 2010). Increased FA, together with decreased $\mathrm{MD}$, has been related to improved motor function in chronic stages after stroke (Lindberg et al., 2007; Lindenberg et al., 2010, 2013; Schaechter et al., 2010). This has also been found for the transcallosal white matter tracts (Lindenberg et al., 2013; van Meer et al., 2012). Furthermore, functional MRI studies have shown post-stroke interhemispheric imbalance as well as altered interhemispheric functional connectivity in human patients and animal models (Carter et al., 2010; Grefkes and Fink, 2011, 2014; van Meer et al., 2010a, $2010 b, 2012)$. Interhemispheric connectivity may improve in chronic stages after stroke, which has been related to behavioural recovery (Carter et al., 2010; Grefkes and Fink, 2011, 2014; van Meer et al., 2010a, 2010b, 2012), thereby also suggesting the importance of transcallosal tract integrity. Despite all this valuable research, still much remains to be elucidated about post-stroke network reorganization, such as the pattern of contralesional remodeling in meso- and macroscale structural networks or the exact role of the CST (i.e. as final central nervous connection to the musculature) in post-stroke network reorganization and recovery.

\section{Brain reorganization after TBI}

$\mathrm{TBI}$ is a very heterogeneous disorder, which is mainly characterized by brain damage due to an external mechanical force (Maas et al., 2008; Xiong et al., 2013). TBI is the leading cause of disability and death in the young adult population up to 45 years of age (Langlois et al., 2009; Roozenbeek et al., 2013). TBI is a complex disease process. After the primary injury, caused by the initial traumatic event, multiple secondary injury mechanisms are involved, such as ischemia, mitochondrial damage, inflammation, and blood-brain barrier damage, which may lead to widespread axonal damage, cell death and ongoing neurodegeneration (Andriessen et al., 2010; Bains and Hall, 2012; Masel and DeWitt, 2010; Xiong et al., 2013). Together, these pathological mechanisms may lead to functional and behavioural deficits, but this can (partially) recover over time, although patients may continue to suffer from symptoms (McMahon et al., 2013).

Anatomical MRI and computed tomography (CT) are conventional imaging modalities used in the clinic to map structural brain damage, such as diffuse injuries, lesions and hematomas (Borg et al., 2004; Maas et al., 2008). However, many patients with TBI-related clinical symptoms show normal appearance on CT and MRI scans of the brain (Belanger et 
al., 2007; Borg et al., 2004; Hofman et al., 2001; Hughes et al., 2004). Recently, studies have used more advanced imaging modalities, such as diffusion MRI and resting-state fMRI to characterize post-TBI brain reorganization. These studies revealed widespread white matter damage throughout the brain. The diffusion MRI parameters FA, MD, AD and RD have shown to be altered after clinical (Bazarian et al., 2007; Edlow et al., 2016; Inglese et al., 2005; Ling et al., 2012; Mayer et al., 2010; Palacios et al., 2012; Rutgers et al., 2008) and experimental TBI (Braeckman et al., 2019; Li et al., 2017a, 2013; Long et al., 2015; van de Looij et al., 2012; Singh et al., 2016; Tu et al., 2016; Wang et al., 2018; Wright et al., 2016; Zhuo et al., 2012). In animal studies, these alterations were shown to be histologically related to axonal injury (Braeckman et al., 2019; Li et al., 2017b, 2013; Singh et al., 2016; Zhuo et al., 2012). Also functional connectivity has been shown to be altered after TBI, which has also been related to underlying white matter integrity (Hillary et al., 2011; Palacios et al., 2012). TBI patients may have decreased functional connectivity, which can partially restore over time (Hillary et al., 2011), a finding that has been replicated in a rat TBI model (Heffernan et al., 2013). Together, these studies suggests that more advanced imaging modalities, such as DTI and BOLD resting-state $\mathrm{fMRI}$ may yield new imaging biomarkers, which may serve as non-invasive diagnostic tools in TBI. However, the exact underlying mechanisms (e.g. axonal damage or excitotoxicity) that drive the spatiotemporal patterns found with imaging markers (e.g. diffusivity parameters), as well as how this relates to behavioural deficits and recovery, needs further elucidation.

\section{Brain reorganization related to ageing}

Over the lifespan the brain develops and ages, which is a highly complex process associated with structural and functional changes across multiple brain regions. First, the volume of the brain changes over the lifespan, which can be described by an inverted U-curve. The grey matter volume decreases linearly after the age of 15 year, whereas the white matter volume starts to decrease somewhere after midlife (Giedd et al., 1999; van Haren et al., 2008; Taki et al., 2011). In line with these changes, DTI studies on ageing have shown decreased FA together with increased MD in white matter, indicative of decreased white matter integrity (Abe et al., 2002; Hsu et al., 2008; Hugenschmidt et al., 2008; Salat, 2011; Salat et al., 2005). These ageing-related grey and white matter changes may cause behavioural problems and cognitive decline. Moreover, ageing is found to be a major risk factor for many diseases and prevalent brain disorders, such as Alzheimer's disease, Parkinson's disease and stroke (Collier and Kordower, 2012; Niccoli and Partridge, 2012). Furthermore, ageing has been shown to influence disease outcome and response to therapies in experimental animal models, which may be related to altered brain organization with ageing (Herson and Traystman, 2014; Liang et al., 2016; Liu et al., 2009). 
Over the last two decades, multiple network studies showed a relationship between healthy ageing and substantial changes in structural (Betzel et al., 2014; Dennis et al., 2013; Gong et al., 2009; Hagmann et al., 2010; Lim et al., 2015; Montembeault et al., 2012; Otte et al., 2015; Wu et al., 2012; Zhu et al., 2012) and functional (Achard and Bullmore, 2007; Andrews-Hanna et al., 2007; Betzel et al., 2014; Meier et al., 2012; Meunier et al., 2009; Spreng and Schacter, 2012; Wang et al., 2012) brain network topology. In general, healthy structural and functional networks have small-world organization, together with hubness and modularity, in both young adult and aged humans (Gong et al., 2009; Zhu et al., 2012). Some studies showed no changes in global efficiency with ageing (Gong et al., 2009), whereas other studies found lower global efficiency in younger subjects (Otte et al., 2015; Wu et al., 2012; Zhu et al., 2012), where global efficiency showed an inverted-U shape across the lifespan (Otte et al., 2015; Wu et al., 2012). Furthermore, the density of structural networks decreases across lifespan (Dennis et al., 2013; Gong et al., 2009; Hagmann et al., 2010), which may be reflective of white matter degeneration in aging brains (Meier-Ruge et al., 1992; Salat et al., 2005). As such, network alterations during ageing may contribute to the vulnerability (or resilience) to brain disorders, which has hardly been systematically investigated so far. Studies on the relationship between network changes and vulnerability to brain disorders in patients are complicated and may be more straightforwardly conducted by lesion simulation studies or in laboratory rodents that age relatively fast and can be scanned longitudinally across the lifespan.

\section{Animal models for stroke and TBI}

Animal models allow for controllable and experimental research, and offer exclusive means to perform longitudinal, multiparametric assessment of whole-brain structural and functional reorganization after brain injuries such as stroke or TBI (Dijkhuizen and Nicolay, 2003; Fluri et al., 2015; Xiong et al., 2013).

Different animal stroke models have been developed. For this thesis, cortical photothrombosis (Watson et al., 1985) and transient middle cerebral artery occlusion (MCAO) models (Garcia et al., 1995; Li et al., 1995; Longa et al., 1989), well-known and widely applied rodent stroke models, were used. The photothrombotic stroke model induces a focal lesion in the sensorimotor cortex, whereas MCAO induces a large subcortical lesion, along with possible cortical damage, dependent on the duration of the occlusion.

To study TBI in this thesis, the Marmarou weight-drop model, a specific model for the acceleration-deceleration event and consequent widespread axonal damage, was used. The model can be easily used to induce different grades of TBI (i.e. by adjusting the drop height) and has been recognized as one of the most relevant TBI models that includes diffuse axonal injury (Finan, 2018; Foda and Marmarou, 1994; Marmarou et al., 1994; Xiong et al., 2013). 


\section{AIMS AND OUTLINE}

The main aim of this thesis was to characterize the spatiotemporal pattern of neuronal reorganization after brain damage due to stroke or traumatic brain injury, as well as to determine the effect of ageing on brain network vulnerability to lesions.

In Chapter $\mathbf{2}$ the structural network topology across the lifespan was assessed with a recently developed network analysis tool, Bayesian exponential random graph modelling, which may resolve several limitations of classical graph analysis. The aim with this approach was to identify the changes in structural network topology (i.e. efficiency, clustering) across the lifespan and to assess the network vulnerability to random and targeted lesion simulations.

In Chapter 3 the aim was to validate whole-brain connectome reconstructions from recent diffusion-MRI based tractography approaches (i.e. CSD-based tractography and global tractography) against neuroanatomical connectivity from neuronal tracer studies. Tractography results were evaluated across different anatomical sites and distances, and the influence of varying tractography parameter settings was examined. In addition, the effects of streamline filtering, streamline thresholding and group-based average network reconstruction and thresholding were evaluated.

In Chapter 4 diffusion-based tractography was applied to map the structural connectivity in the contralesional hemispheric chronically after stroke in rats. The structural network backbone topology was assessed with the minimum spanning tree (MST) approach, and network topologies of stroke and control animals were examined. In addition, a possible relationship between structural network properties and behavioural performance chronically after stroke was investigated.

In Chapter $\mathbf{5}$ the aim was to determine the relationship between age-related neural network characteristics and stroke outcome. To that aim local and global network topology features were characterized, and hub regions from structural and functional networks were identified in young adult and aged rats. Subsequently, the effects of simulated lesions on structural and functional network features, as well as on the hub-status of specific regions, were investigated. Moreover, the effect of ageing on post-stroke network alterations was tested by comparing the network modifications in young adult and aged rats.

In Chapter 6 it was investigated how two important white matter tracts involved in sensorimotor function and interhemispheric communication, i.e. the CST and corpus callosum, are spatiotemporally affected after experimental focal stroke, and whether this is related to functional connectivity and sensorimotor performance. Therefore diffusionbased characteristics of white matter tracts in the CST and corpus callosum, functional connectivity and behavioural performance were measured over time.

In Chapter $\mathbf{7}$ spatiotemporal patterns of structural and functional changes in grey and white matter regions were characterized from acute to chronic stages after mild and 
moderate TBI. An additional aim was to identify early imaging markers from more advanced imaging techniques, such as diffusion and resting-state MRI, which may predict post-TBI outcome better than conventional MRI techniques, such as $T_{2}$-weighted MRI. In addition, structural and functional MRI findings were related to behavioural performance and validated with histological evaluations.

In Chapter 8 the findings from Chapters 2-7 are summarized and discussed. In addition, several pitfalls and potential future directions are outlined. 


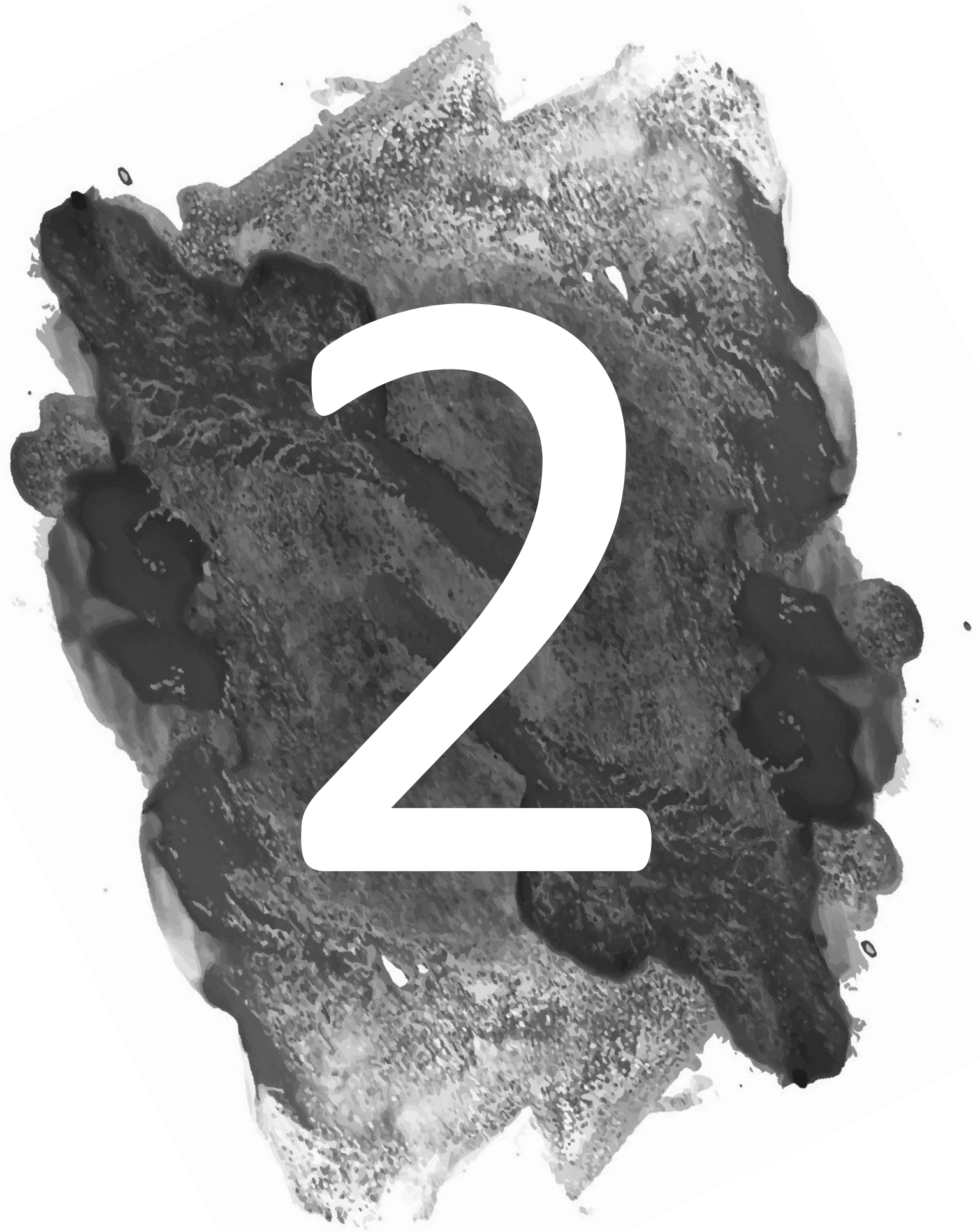




\section{Chapter 2}

\section{Bayesian Exponential Random Graph Modelling of Whole-Brain Structural Networks across Lifespan}

M.R.T. Sinke, R.M. Dijkhuizen, A. Caimo, C.J. Stam \& W.M. Otte 


\section{ABSTRACT}

Descriptive neural network analyses have provided important insights into the organization of structural and functional networks in the human brain. However, these analyses have limitations for inter-subject or between-group comparisons in which network sizes and edge densities may differ, such as in studies on neurodevelopment or brain diseases. Furthermore, descriptive neural network analyses lack an appropriate generic null model and a unifying framework. These issues may be solved with an alternative framework based on a Bayesian generative modeling approach, i.e. Bayesian exponential random graph modeling (ERGM), which explains an observed network by the joint contribution of local network structures or features (for which we chose neurobiologically meaningful constructs such as connectedness, local clustering or global efficiency). We aimed to identify how these local network structures (or features) are evolving across the life-span, and how sensitive these features are to random and targeted lesions. To that aim we applied Bayesian exponential random graph modeling on structural networks derived from whole-brain diffusion tensor imaging-based tractography of 382 healthy adult subjects (age range: 20.2 -86.2 years), with and without lesion simulations. Networks were successfully generated from four local network structures that resulted in excellent goodness-of-fit, i.e. measures of connectedness, local clustering, global efficiency and intrahemispheric connectivity. We found that local structures (i.e. connectedness, local clustering and global efficiency), which give rise to the global network topology, were stable even after lesion simulations across the lifespan, in contrast to overall descriptive network changes - e.g. lower network density and higher clustering - during aging, and despite clear effects of hub damage on network topologies. Our study demonstrates the potential of Bayesian generative modeling to characterize the underlying network structures that drive the brain's global network topology at different developmental stages and/or under pathological conditions. 


\section{INTRODUCTION}

Aging is a major risk factor of prevalent diseases in society, including neurodegenerative disorders such as Alzheimer's and Parkinson's disease (Collier and Kordower, 2012; Niccoli and Partridge, 2012). During aging the human brain is subject to structural and functional changes that can cause behavioral problems and cognitive decline (e.g. reduced executive functioning or memory impairment). However, many elderly people do not suffer from behavioral and cognitive problems and are functioning well, despite structural and functional changes in brain networks. Therefore it is important to understand to what extent specific changes in brain connectivity across the lifespan contribute to increased risk and development of age-related neurological disorders, even in the absence of significant brain pathology (Burke and Barnes, 2006).

Graph analysis has proven to be an elegant tool to assess topological aspects of structural and functional connectivity in the brain (Bullmore and Sporns, 2009). Graph analysis describes the brain as a set of nodes, representing neural elements, linked by edges, representing some measure of structural, functional or causal interaction between the nodes. Many studies have successfully applied graph analysis to capture network topologies with either individual or aggregated node metrics (e.g. the average shortest path length, maximum betweenness centrality or overall clustering coefficient) (Bullmore and Sporns, 2009) and/or network properties such as small-worldness, rich club connectedness (Bullmore and Sporns, 2012; Cao et al., 2014) and modularity (Rubinov and Sporns, 2010). In the past decade, multiple studies have shown that normal aging is associated with substantial alterations in structural (Betzel et al., 2014; Dennis et al., 2013; Gong et al., 2009; Hagmann et al., 2010; Lim et al., 2015; Montembeault et al., 2012; Otte et al., 2015; Wu et al., 2012; Zhu et al., 2012) and functional (Achard and Bullmore, 2007; Andrews-Hanna et al., 2007; Betzel et al., 2014; Meier et al., 2012; Meunier et al., 2009; Spreng and Schacter, 2012; Wang et al., 2012) brain networks. Some of these studies focussed on specific age categories: childhood to adulthood (Dennis et al., 2013; Hagmann et al., 2010) or young and older adults (e.g. (Meunier et al., 2009; Zhu et al., 2012)). From childhood to adulthood a decrease in path length and clustering, and increase in efficiency have been observed (Dennis et al., 2013; Hagmann et al., 2010) which may differ between the hemispheres (Dennis et al., 2013). Other studies have shown a higher local clustering and lower global efficiency in older adults compared to younger adults (Zhu et al., 2012), where modularity decreases across networks (Meunier et al., 2009). In line with these findings several studies have shown inverted-U shaped global efficiency across lifespan (Otte et al., 2015; Wu et al., 2012). Functional connectivity assessment has revealed increased integration and decreased randomness, whereas connectivity decreased significantly during adulthood (Smit et al., 2016). Despite these significant network changes, throughout development brain networks largely maintain small-world properties, modularity and stable hub-regions 
(Dennis et al., 2013; Gong et al., 2009; Hagmann et al., 2007). In general, the aging brain can be characterized by reduced centrality of hub regions with a decrease in global efficiency and an increase in local network clustering. Similar changes in hub regions and subsequent effects on global efficiency have also been characterized in various neurological disorders (Crossley et al., 2014; Stam, 2014).

Despite the popularity of descriptive graph analysis, it has non-trivial intrinsic limitations, particularly for intersubject or between-group comparisons where networks have different sizes, densities and degree distributions (Fornito et al., 2013; van Wijk et al., 2010). The most commonly used node metrics, like the clustering coefficient and path length, are highly dependent on the total number of connections and the average degree of a network (Stam et al., 2014; van Wijk et al., 2010). This hampers comparability of brain network topology across the human lifespan, as network densities substantially differ between ages (Dennis et al., 2013; Gong et al., 2009; Hagmann et al., 2010), which may be explained by changes in the integrity of connecting fibers or the cortical density of neurons (Salat, 2011; Westlye et al., 2010).

A second limitation is the lack of an appropriate generic null model to test the significance of a particular network measure against. A frequently used null model is a network with randomly shuffled edges that shares basic characteristics with the measured network, like degree distribution, size and density. Different network metrics require distinct null models if compared between networks (e.g., networks with asymmetric degree distributions cannot be explained by the Watts-Strogatz small-world network null model but require the Barabási-Albert scale-free model) (Fornito et al., 2013).

A third limitation of graph analysis is the type I error inflation if multiple network nodes are compared within the same brain, or if different network metrics are calculated from a single network.

Fourthly, graph analysis consists of univariable comparisons (i.e., network metrics are determined independent from each other) due to lack of a unifying framework (Telesford et al., 2011). However, many metrics are highly correlated and non-exclusive (Bounova and de Weck, 2012; Meghanathan, 2015).

A promising alternative analysis approach, which may in theory overcome the abovementioned limitations in descriptive graph analysis, is the framework of generative modeling (Fornito et al., 2013; Klimm et al., 2014), which aims to condense a complex network topology into a parsimonious description (i.e. mathematical equation). Growth models are a relatively well known class of generative models. They involve growing of artificial networks via addition of nodes and edges and rewiring of existing edges according to pre-specified mechanisms, and comparing topologies between these artificially grown and observed networks. Relatively simple growing mechanisms (i.e., the mathematical local structures) provide a generative model that allows growing of networks that closely 
resemble observed brain networks. A recent successful example is a growth model with two local structures: a combined distance penalty based on the cost of maintaining long-range connections and a topological term that favors links between regions sharing similar input (Vertes et al., 2012). Similar principles have been successfully applied by other recent studies on neural networks (Betzel et al., 2015; Goni et al., 2014). However, unambiguous determination of distances between non-connecting pairs of network nodes - required for distance penalties - is difficult.

Another recent and powerful class of generative models are the exponential random graph models. Their usefulness has been emphasized in social network studies (Robins et al., 2007a), but they may have equal potential for neuronal networks (Simpson et al., 2011). Until recently, exponential random graph models have been difficult to handle from a statistical point of view, due to the intractability of the normalizing constant and the problem of model degeneracy (Handcock, 2003), which has limited their applicability. The recent presentation of a Bayesian inference framework, using adaptive Markov chain Monte Carlo approaches to fit exponential random graph models, mitigated the issue of model degeneracy and significantly improved fitting performance (Caimo and Friel, 2011). Exponential random graph models are able to explore multiple local network features (e.g. connectedness, local clustering or global efficiency) simultaneously and assess how these local features give rise to the global network topology, thereby taking into account their mutual dependencies (note that the term 'local' is defined from a topological and not from a physical perspective, i.e. local clustering does not necessary imply physical proximity of involved nodes). In addition, exponential random graph models inherently account for bias due to density differences (van Wijk et al., 2010). More technically, the models capture the joint probability of a (global) network $G$, governed by $\boldsymbol{\vartheta}$, a set of network parameters (e.g. local clustering, edges) of a postulated generative process. If $\boldsymbol{\vartheta}$ is estimated well, synthetic networks - which are structurally similar to $G$ - may be drawn from a probability distribution $P(G \mid \vartheta)$. Exponential random graph modeling may thus also be considered as a mathematical framework to condense the (global) topological information of a network into a limited set of parameters (i.e. the local network structures or features). This mathematical description theoretically provides: $i$ ) compression of the observed network data into a basic equation, ii) capturing of the most relevant patterns within the observed network, iii) generalization from the observed network to unobserved networks of the same type, iv) generalization across network sizes, and $v$ ) prediction of network topologies.

Exponential random graph models may provide unambiguous answers to fundamental questions related to brain-wide network organization and changes across lifespan, such as: how do local network features (i.e. neurobiologically meaningful constructs such as local clustering, connectedness and global efficiency) simultaneously give rise to (i.e. explain) the global network topology, and what is the relative significance (i.e. contribution) of those 
local structures during development and aging? Will there still be changes across lifespan in brain global efficiency or local clustering, if confounding effects such as correlations between metrics and decreased network density with increased age, are effectively taken into account? In fact, surprisingly little is known on how local network features simultaneously shape the global network characteristics so commonly reported in descriptive graph analysis studies. Furthermore, it is unknown to what extent local structures are affected by specific network damage in brain injuries and pathologies. For example, does damage to central hub regions result in distinct local features (such as brain network clustering or connectedness) as compared to diffuse network damage, and does this differ across the lifespan?

Our study objective was to characterize the relative contributions of local network structures that might explain the configuration of structural whole-brain networks across the human lifespan. To that aim we fitted Bayesian exponential random graph models on structural networks, obtained from diffusion tensor imaging (DTI) data from 382 healthy subjects in the age range of 20 to 86 years. As these models are based on probability estimates, we were able to directly compare the optimally fitted parameters that explain the network topology between different age groups. Furthermore we evaluated in simulations the consequences of hub lesioning and diffuse network damage on the local structures across the human lifespan. We hypothesized that Bayesian exponential random graph modeling could characterize the observed networks with a limited set of network parameters, and would allow generation of synthetic networks that are topologically similar to the observed networks. Based on findings from descriptive graph analysis in healthy subjects (Otte et al., 2015) we expected: $i$ ) a decrease in contribution of global network efficiency structures and density (e.g. total number of edges) with age, ii) an increase in local clustering structures with age, and iii) significant changes in relative contributions of local substructures when hub nodes are damaged.

\section{METHODS}

\section{Dataset}

Standardized high quality, DTI and $\mathrm{T}_{1}$-weighted datasets were obtained from the 'Information extraction from images' database (http://biomedic.doc.ic.ac.uk/braindevelopment/), which is freely available. We included scans from 382 healthy adults recruited from the general United Kingdom population. The age range was 20.2 to 86.2 years. This DTI dataset has been analyzed previously to characterize the backbone of the structural whole-brain network across the lifespan (Otte et al., 2015).

Subjects were scanned once with a 1.5T (205 subjects, Philips Medical Systems Gyroscan Intera) or 3T (177 subjects, Philips Medical Systems Intera). Ethical approval was given by the Thames Valley multicenter ethics committee. Images were acquired using a single-shot 
echo-planar (EPI) DTI sequence. Imaging parameters were: 15 diffusion-weighted images with $b=1000 \mathrm{~s} / \mathrm{mm}^{2}, 2$ images without diffusion-weighting, 56 axial slices, $2.35 \mathrm{~mm}$ slicethickness, $128 \times 128$ acquisition matrix, $1.75 \times 1.75 \mathrm{~mm}$ voxels; repetition time (TR)/echo time (TE) $9.1 \mathrm{~s} / 80 \mathrm{~ms}$ (at $1.5 \mathrm{~T}$ ) or $11.9 \mathrm{~s} / 51 \mathrm{~ms}$ (at 3T). In addition, $\mathrm{T}_{1}$-weighted images were acquired using a spin-echo sequence with the following parameters: $8^{\circ}$ flip angle, TR/TE 9.6/4.6 ms (at 1.5T) or TR/TE 9.8/4.6 ms (at 3T), 150 axial slices, $1.2 \mathrm{~mm}$ slice-thickness, 256 $\times 256$ acquisition matrix, $0.94 \times 0.94 \mathrm{~mm}$ voxels.

\section{Image processing}

All diffusion-weighted images were corrected for subject motion using FLIRT followed by Bmatrix adjustment using the affine registration parameters. Within-subject diffusionweighted and $\mathrm{T}_{1}$-weighted images were non-rigidly aligned, and subsequently registered to a $\mathrm{T}_{1}$-weighted reference image that was matched to the Harvard-Oxford atlas (Desikan et al., 2006). Using the $50 \%$ probability threshold Harvard-Oxford cortical parcellation masks, we partitioned each hemisphere in 48 cortical network regions, i.e. 96 network regions bilaterally, based on standard anatomical boundaries.

Analysis of DTI data was performed using the Diffusion Toolkit (Wang et al., 2007). Diffusion images were resampled on an isotropic $1.75 \mathrm{~mm}$ grid, and fractional anisotropy (FA) maps were calculated based on voxel-wise estimates of the diffusion tensor. Wholebrain tractography was obtained from seeds in each voxel according to FA $>0.2$ using the interpolated streamline algorithm implementation (Euler integration method with step size of $0.5 \mathrm{~mm}$ ), with streamlines originating from ten random seeds in each voxel, a maximal $70^{\circ}$ angle threshold and minimal FA threshold of 0.2 (DtiStudio version 0.6). The algorithm is based on the Fiber Assignment by Continuous Tracking (FACT) approach, by which tracking is performed using a continuous coordinate system rather than a discrete voxel grid. Structural connections between any pair of cortical network regions were identified from streamlines that had end points in both regions.

The image processing pipeline is schematically illustrated in Figure 1.

\section{Construction of whole-brain structural networks}

For each of the 382 sets of whole-brain structural connections we constructed a binary undirected network, described by the graph $G=(N, E)$ where $N$ is the set of 96 bilateral cortical network regions and $E$ is the set of edges $e_{i j}$ in the $N \times N$ adjacency matrix, with $e_{i j}$ set to 1 if region pairs were connected with one or more streamlines, or 0 otherwise. No self-connections were allowed. 


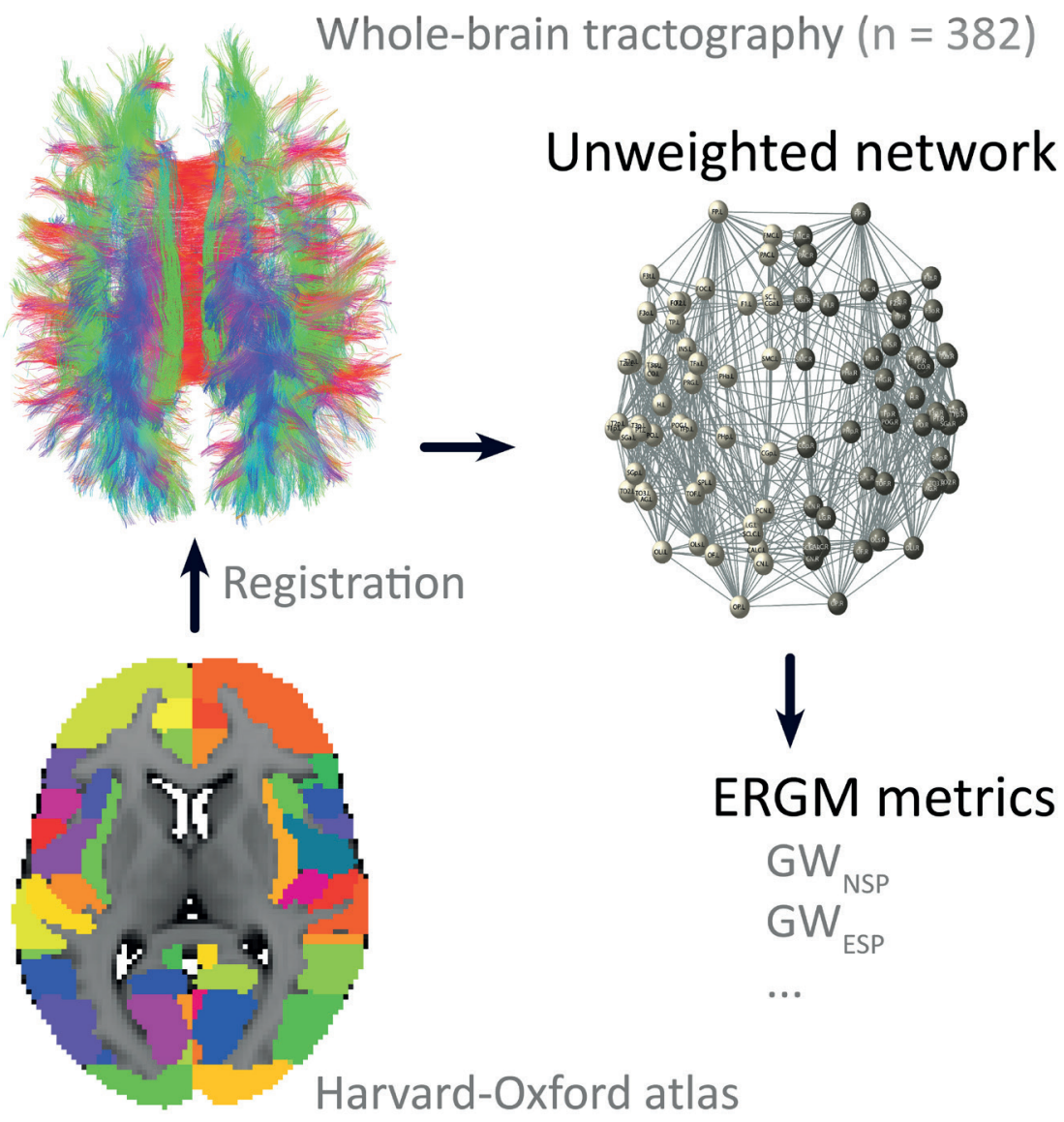

Figure 1. Schematic overview of the image processing pipeline. Diffusion-weighted and $\mathrm{T}_{1}$-weighted images were non-rigidly aligned and subsequently registered to a $T_{1}$ reference scan matched with the Harvard-Oxford atlas (50\% probability threshold, 96 bilateral cortical regions). Whole-brain tractography was performed using an Euler integration method (FA $>0.2,70^{\circ}$ angle threshold). For all 382 subjects binary undirected networks were constructed, where a connection is set to 1 if region pairs were connected with one or more streamlines, or 0 otherwise. Graphs were averaged into four age-category networks. ERGM metrics (e.g. $\mathrm{GW}_{\mathrm{ESP}}, \mathrm{GW}_{\mathrm{NSP}}$ ) were calculated from these networks.

Participants were divided into different age-categories: 20-34, 35-50, 51-70 and >70 years. For each age category individual graphs were summed and divided by the total number of participants within each age category, resulting in an average (weighted) connectivity matrix that shows the proportion of participants having a specific connection for each node pair (Figure S1). Edges in the age-category graphs were defined as present if 
$>35 \%$ of subjects within that age range contained an edge in their individual network (Figure S2). By using this $35 \%$ threshold we included at least $25 \%$ of prevalent connections over all age categories. All subsequent analyses were based on these binary age-category graphs.

The 'hubness' of individual nodes - required for the network damage simulations - was calculated as the betweenness centrality and ranked from high to low (i.e., central to peripheral centrality) (Rubinov and Sporns, 2010). Hub nodes have a high betweenness centrality, i.e. a relatively high number of shortest paths passing through the network nodes. The betweenness centrality of node $i$ is defined as

$$
b c_{i}=\frac{1}{(n-1)(n-2)} \sum_{j \neq k, k \neq i, j \neq i}^{n} \frac{g_{j k}(i)}{g_{j k}} \text { where } g_{j k} \text { is the shortest path between a pair of }
$$

other nodes and $g_{j k}(i)$ is the number of those node paths that pass through node $i$.

\section{Bayesian exponential random graph modeling}

Exponential random graph models constitute a broad class of network models that assume that the topological structure of an observed network $x_{o b s}$ can be explained in terms of the relative contribution of a set of mutual dependent local structures or features $g(x)$ (e.g. clustering coefficient, path length, location in the brain). The models require a defined network distribution $\chi^{(n)}$ that contains all possible binary networks with size $n$. In our study, $n$ was 96, the number of atlas regions. A network can then be represented by a random variable $\boldsymbol{X}$. A realization of $\boldsymbol{X}$ is denoted by $x=\left\{x_{i j}\right\}$. An observed network (e.g. one of the four age-category graphs) $x_{o b s}$ is regarded as just one realization from the set of all possible networks $\boldsymbol{X}$ with similar network topology. In other words, $x_{o b s}$ is assumed to be the outcome of some stochastic process. The goal of exponential random graph models is to propose a plausible model for this stochastic process (Frank and Strauss, 1986; Pattison and Wasserman, 1996). More formally, exponential random graph models are a particular class of discrete linear exponential families that represent the probability distribution of $\boldsymbol{X}$ as

$$
\pi(X=x \mid \theta)=\frac{\exp \left\{\theta^{t} g(x)\right\}}{z(\theta)}
$$

where $\theta \in \Theta \subset \Re^{q}$ is the vector of model coefficients (that has to be estimated) related to $g(x)$ which quantifies the relative contribution (i.e. significance) of each local network feature (e.g. local clustering, global efficiency) in explaining the global structure of $x_{o b s \text {, }}$ thereby taking into account the contribution of other network features in the model. The $\vartheta$ represents the change in log-odds of an edge existing for each unit increase for a specific 
explanatory parameter (i.e. clustering) in the model. If $\boldsymbol{\vartheta}$ is large and positive for this specific local structure, then this metric plays an important role in explaining the global network structure (i.e. local clustering contributes more to the network than you would expect by chance). If $\boldsymbol{\vartheta}$ is large and negative, then this metric also plays an important role in explaining the overall network topology, but is less important (i.e. there is less local clustering) than expected by chance. Furthermore, $z(\theta)=\sum_{x \in \chi} \exp \left\{\theta^{t} g(x)\right\}$ is the normalizing constant which is generated over the entire network space $\chi^{(n)}$ to ensure that (1) is a proper probability distribution. Since $\chi^{(n)}$ consists of $2^{n \times n}$ possible networks, the normalizing constant $z(\vartheta)$ (i.e. ensures that the probabilities sum to one) is intractable. To avoid the need to calculate $z(\boldsymbol{\vartheta})$, a pseudolikelihood estimation method has been proposed (Strauss and Ikeda, 1990). Although fitting exponential random graphs using this pseudolikelihood estimation is computationally relatively easy, the properties of the estimator are not well understood and the estimated $\vartheta$ are known to be inaccurate in many cases (Robins et al., 2007b). An alternative and more straightforward method to fit exponential random graph models is by means of Monte Carlo maximum likelihood estimation (Hunter and Handcock, 2006). This form of estimation simulates a distribution of random networks from a starting set of model parameters, $\boldsymbol{\vartheta}_{0}$, and subsequently refines the parameter values by comparing the distribution of networks against the measured network $x_{o b s}$. A crucial aspect of this estimation is a proper choice of $\boldsymbol{\vartheta}_{0}$. Poorly chosen initial values may lead to nonconvergence or degenerative probability distributions, and thus result in unreasonable estimates of $\boldsymbol{\vartheta}$. For details on this issue see (Handcock, 2003) and (Fienberg et al., 2009).

Recently a Bayesian solution for this issue of proper initialization of $\boldsymbol{\vartheta}$ was proposed and successfully tested in the area of social network modeling (Caimo and Friel, 2011; Caimo and Mira, 2014). In this method a prior distribution $\pi(\boldsymbol{\vartheta})$ is assigned to $\boldsymbol{\vartheta}$. The posterior distribution $\pi(\theta \mid x) \propto \pi(x \mid \theta) \pi(\theta)$ is subsequently solved with a Monte Carlo maximum likelihood method based on the exchange algorithm (Murray et al., 2006). This Bayesian framework has been shown to perform very well, and was used in the current study as the method of choice in fitting exponential random graph models.

\section{Local structure definitions}

A large set of local structures are provided in the literature to use with exponential random graph models (Hunter et al., 2009). Two previous studies (Simpson et al., 2011, 2012) included local structures that resemble most of the descriptive network characteristics used in classical graph analysis studies, i.e. connectedness, local clustering and global efficiency (Bullmore and Sporns, 2009; Morris et al., 2008; Rubinov and Sporns, 2010). These studies showed that three local structures or features, i.e. the non-edgewise share partners, edgewise shared partners and edges, contributed significantly in most functional brain 
networks (Simpson et al., 2011, 2012). Although we investigated structural rather than functional networks, we used the same structures for our analyses; first, because many (classical) graph analysis studies have also used similar graph metrics (e.g. clustering coefficient, path length, modularity) to describe both functional and structural networks; second, because there is a strong correlation between structural and functional networks; and third, because it has been shown that structural connections are predictive of functional connections (Hagmann et al., 2007; Honey et al., 2009; Sporns, 2010). In addition, we included a local structure based on the hemispheric attribution of a node (i.e. whether a node tended to make more connections within or between hemispheres) as the white matter connectivity within hemispheres is found to be higher in comparison to the connectivity between hemispheres (Hagmann et al., 2008). Figure 2 shows a graphical representations of local structures used in network generation. These were defined as:

The number of edges

$$
s(x)=\sum_{i<j} x_{i j}
$$

A measure of connectedness compatible with exponential random graph modeling, i.e. an increase in the edge coefficient means an increase in number of connections, or a higher network density.

The geometrically weighted edgewise shared partner (GW $W_{E S P}$ )

$$
w\left(x, \phi_{v}\right)=e^{\phi w} \sum_{k=1}^{n-2}\left\{1-\left(1-e^{-\phi w}\right)^{k}\right\} E P_{k}(x)
$$

With $E P_{k}(x)$ defined as the number of pairs $\{i, j\}$ such that $x_{i j}=1$ and $i$ and $j$ have exactly $k$ common neighbors, which is a measure of local clustering compatible with exponential random graph modeling. An increase in GWESP coefficient means more local clustering (segregation). In other words, two connected nodes (brain regions) tend to connect to similar other brain regions (i.e. shared partners).

The geometrically weighted non-edgewise shared partner (GW $\left.W_{N S P}\right)$

$$
p\left(x, \phi_{p}\right)=e^{\phi p} \sum_{k=1}^{n-2}\left\{1-\left(1-e^{-\phi p}\right)^{k}\right\} N P_{k}(x)
$$

With $N P_{k}(x)$ defined as the number of pairs $\{i, j\}$ such that $x_{i j}=0$ and $i$ and $j$ have exactly $k$ common neighbors, which is a measure of global efficiency compatible with exponential

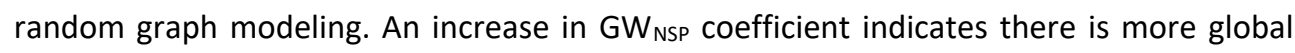
efficiency (integration); an increase in non-connected nodes, sharing one or more connections to the same brain regions (i.e. shared partners). This could be more distant 
brain regions which are indirectly connected (i.e. via a shared partner), or a so-called connector-hub, connecting two network modules (Bullmore and Sporns, 2009).

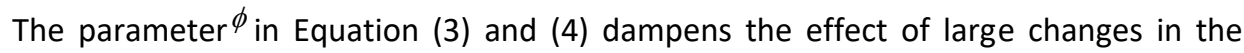
statistics of higher $k$ and was in this study fixed to 0.75 so that the model remains a regular exponential random graph model (Hunter and Handcock, 2006).

The hemispheric nodematch

A binary nodematch-local structure, called hemispheric nodematch, captured whether two nodes belong to the same hemisphere (left or right).

$$
h(x)=\sum_{i<j} x_{i j} x_{j i}: m_{i}=m_{j}
$$

With $m$ as the hemisphere membership.

\section{Summary estimates}

The Bayesian exponential random graph models provide full probability density estimates. We summarized the posterior probability density estimates of the $\boldsymbol{\vartheta}$ coefficients - which correspond with the local structures - as the mean and $95 \%$ credibility intervals.

A

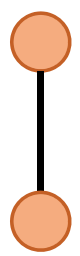

B

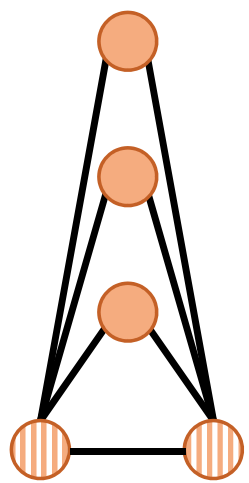

C

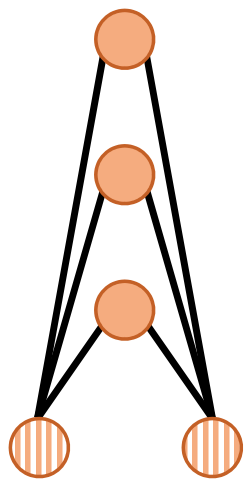

D

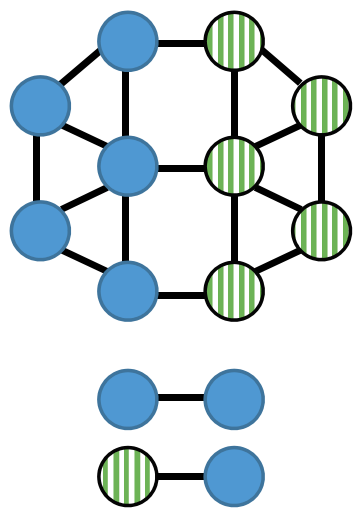

Figure 2. Local structures used in network generation. (A) Edges, a connection between two nodes (measure of connectivity), (B) $\mathrm{GW}_{\mathrm{ESP}}$, weighted sum of number of connected nodes (striped) having exactly $i$ shared partners (measure of local clustering), (C) GW NSP, weighted sum of number of nonconnected nodes (striped) having exactly I shared partners (measure of global efficiency), (D) Hemispheric nodematch, where blue-blue (filled-filled) connections represent within hemisphere connections and green-blue (striped-filled) connections represent between hemisphere connections.

\section{Goodness-of-fit}

We assessed the data goodness-of-fit to the posterior model by comparing the network data $y$ by means of a set of network simulations $\left(y_{1}, y_{2}, \ldots y_{s}\right)$ from $S$ independent realizations 
$\left(\boldsymbol{\vartheta}_{1}, \boldsymbol{\vartheta}_{2}, \ldots \boldsymbol{\vartheta}_{s}\right)$ of the posterior density estimate. We then compared the observed network with the simulated network based on three global descriptive network characteristics: degree (connectivity), geodic distance (shortest path) and edge-wise shared partners (clustering) from the Bergm package (Caimo and Friel, 2014). Additionally, we implemented the triad census (i.e., subset of motifs), that determines the contributions, as a probability, of one, two or three connections between all possible node triples (Hunter et al., 2009; Morris et al., 2008), and compared these contributions between the observed and simulated networks.

\section{Effect of simulated network damage}

We estimated the effect of network damage on the local structures/features by simulating lesions in our data. First we simulated lesions by randomly eliminating $5 \%$ to $25 \%$ of the nodes and connecting edges in steps of $5 \%$. We applied the same procedure for the hubnodes, removing increasing percentages of nodes with the highest betweenness centrality. We evaluated the consequences of random-node and hub-node damage on the local structure parameters (e.g. edges, GW $\mathrm{ESP}_{\mathrm{ES}}, \mathrm{GW}_{\mathrm{NSP}}$, and hemispheric nodematch). The effect of simulated damage between percentages of eliminated nodes was quantified with $95 \%$ credibility intervals obtained from the difference between the posterior distributions.

Vulnerability to hub-node elimination - defined as a change in local substructure parameter between $0 \%$ and $25 \%$ eliminated nodes - was subsequently compared between age categories based on Bayes Factors obtained from a Bayesian equivalent of an independent two sample t-test (Rouder et al., 2009). Bayes factors give the ratio of model likelihoods, thereby providing which model (i.e., the presence or absence of a difference in the parameter change) is supported (i.e., more likely to occur) (Caimo and Friel, 2011). The interpretation of Bayes factors is given in Table 1.

\begin{tabular}{ll}
\hline $\log$ (Bayes factor) & Interpretation \\
\hline$<-5$ & very strong support for baseline model \\
-5 to -3 & strong support for baseline model \\
-3 to -1 & positive support for baseline model \\
-1 to 0 & weak support for baseline model \\
0 & no support for baseline model \\
0 to 1 & weak support for alternative model \\
1 to 3 & positive support for alternative model \\
3 to 5 & strong support for alternative \\
$>5$ & very strong support for alternative model \\
\hline
\end{tabular}

Table 1. Log transformed Bayes factors and their interpretation in the comparison of a baseline and alternative model (Raftery, 1995). 


\section{Statistical analyses}

All network analysis, statistical modeling and visualization was performed in $\mathrm{R}$ (http://www.r-project.org/) using the open-source packages igraph, Bergm, BayesFactor, network and ggplot2.

\section{RESULTS}

\section{Network matrices and model parameters}

We generated group-based networks for each age category, which are depicted in Figure $\mathbf{3}$ as network graph representations (for binary adjacency matrices, see Figure S3). Exponential random graph models were estimated for each age category with the four local network structures edges, $\mathrm{GW}_{\mathrm{NSP}}, \mathrm{GW}_{\mathrm{ESP}}$ and hemispheric nodematch. All local structures were found to contribute significantly to the global network topology. Parameter coefficients and corresponding credible intervals are given in Table 2. Goodness-of-fit measures showed that the simulated networks matched well with the observed networks for all age categories. The goodness-of-fit metrics (i.e. degree, geodesic distance and edgewise shared partners) and triad census contributions of simulated networks almost completely matched with the metrics of the observed networks, as seen in Figure 4 and Figure S4, respectively.
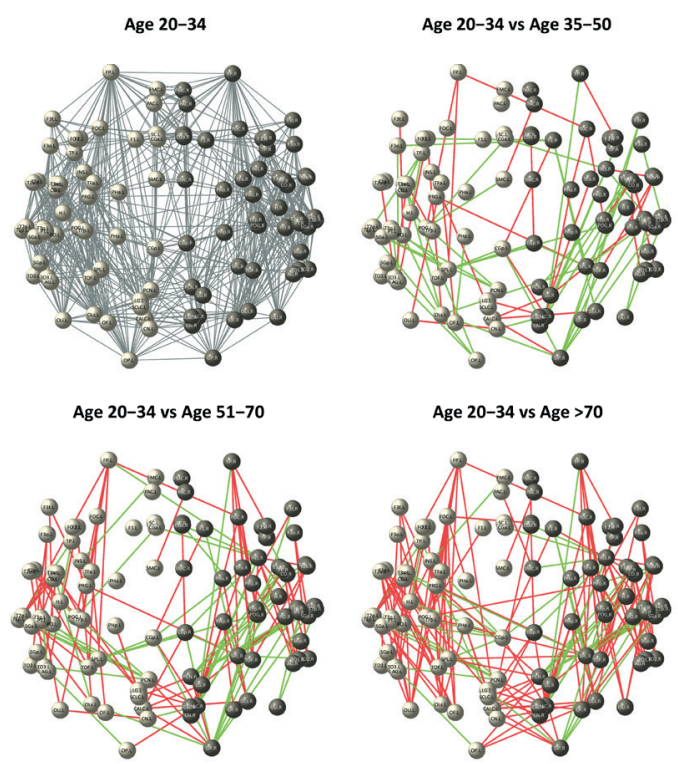

Figure 3. Graph changes across lifespan. Top left shows the network graph for age 20-34. Other graphs represent network differences with age 20-34 for each additional age. Red lines represent 'lost connections' and green lines represent 'new connections'. Density of networks change across the lifespan with $+1.6 \%$ (age 35-50), $-4.4 \%$ (age 51-70) and $-8.0 \%$ (age $>70$ ). 


\begin{tabular}{|c|c|c|c|c|}
\hline \multirow[b]{2}{*}{$\begin{array}{l}\text { Age } \\
\text { category }\end{array}$} & \multicolumn{4}{|c|}{ Model Parameter Coefficients } \\
\hline & Edges & $\mathbf{G W}_{\mathrm{ESP}}$ & $\mathbf{G W}_{\text {NSP }}$ & $\begin{array}{l}\text { Hemispheric } \\
\text { nodematch }\end{array}$ \\
\hline $20-34$ & $\begin{array}{l}-2.38 \\
(-3.45--1.31)\end{array}$ & $\begin{array}{l}1.52 \\
(1.04-1.99)\end{array}$ & $\begin{array}{l}-0.27 \\
(-0.34--0.20)\end{array}$ & $\begin{array}{l}-0.68 \\
(-1.32--0.05)\end{array}$ \\
\hline $35-50$ & $\begin{array}{l}-2.36 \\
(-3.41--1.32)\end{array}$ & $\begin{array}{l}1.49 \\
(1.02-1.96)\end{array}$ & $\begin{array}{l}-0.27 \\
(-0.34--0.20)\end{array}$ & $\begin{array}{l}-0.72 \\
(-1.35--0.09)\end{array}$ \\
\hline $51-70$ & $\begin{array}{l}-2.41 \\
(-3.43--1.39)\end{array}$ & $\begin{array}{l}1.53 \\
(1.06-1.99)\end{array}$ & $\begin{array}{l}-0.27 \\
(-0.34--0.20)\end{array}$ & $\begin{array}{l}-0.75 \\
(-1.36--0.15)\end{array}$ \\
\hline$>70$ & $\begin{array}{l}-2.61 \\
(-3.58--1.64)\end{array}$ & $\begin{array}{l}1.63 \\
(1.20-2.06)\end{array}$ & $\begin{array}{l}-0.25 \\
(-0.31--0.18)\end{array}$ & $\begin{array}{l}-0.89 \\
(-1.53--0.24)\end{array}$ \\
\hline
\end{tabular}

Table 2. Mean model parameter coefficients and $95 \%$ credible (i.e. posterior probability) intervals for each age category.

\section{Global and local network features}

Clear alterations in network topology were found in the older age categories compared with the youngest age category of 20 to 34 years (Figure 3). Across the lifespan new connections are found and other connections are lost (represented by green and red lines respectively), resulting in density changes. However, the relative contribution of local network structures was stable across age (Figure 5). Using different thresholds for constructing group based binary networks (Figures S5 and S6) affected the edges coefficient. This was expected as different thresholds result in different network densities. The edges coefficient captures the density. Different thresholds hardly affected the other coefficients (Figure S7). Furthermore, using an average of models fitted on ten individual networks, randomly picked from each age category, and resulted in similar coefficients as found with the group-based networks. 

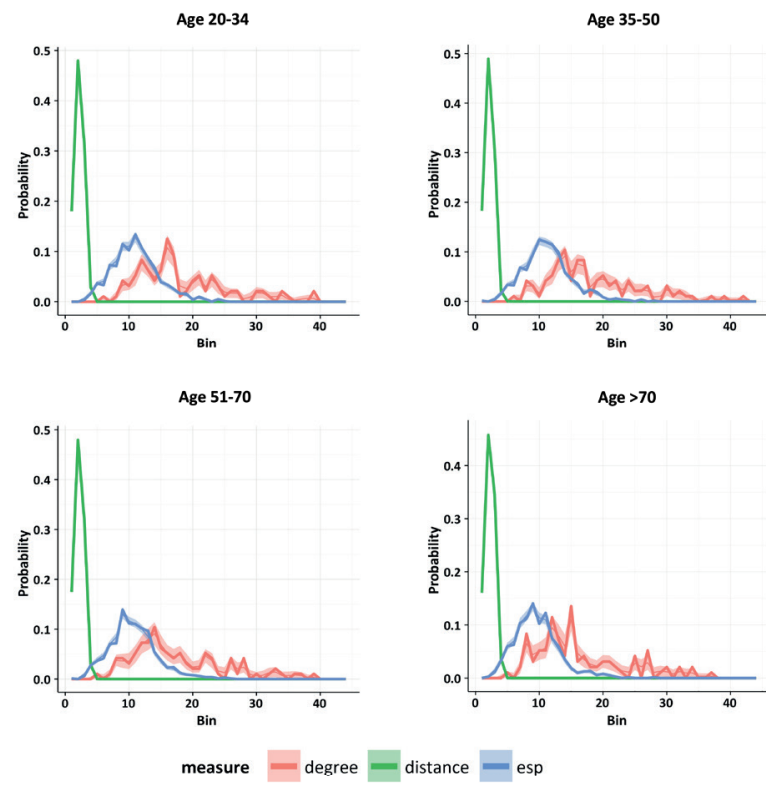

Figure 4. Goodness of fit of simulated networks. Bayesian goodness of fit statistics for simulated networks for all age categories. The red, green and blue lines represent degree (connectivity), distance (shortest path length) and edge-wise shared partners (local clustering), respectively. Bold lines represent the observed probabilities (i.e. proportions of nodes), and thin lines represent the 'averages' of simulated networks, drawn from the posterior distribution, plotted with standard deviations (transparent bands).
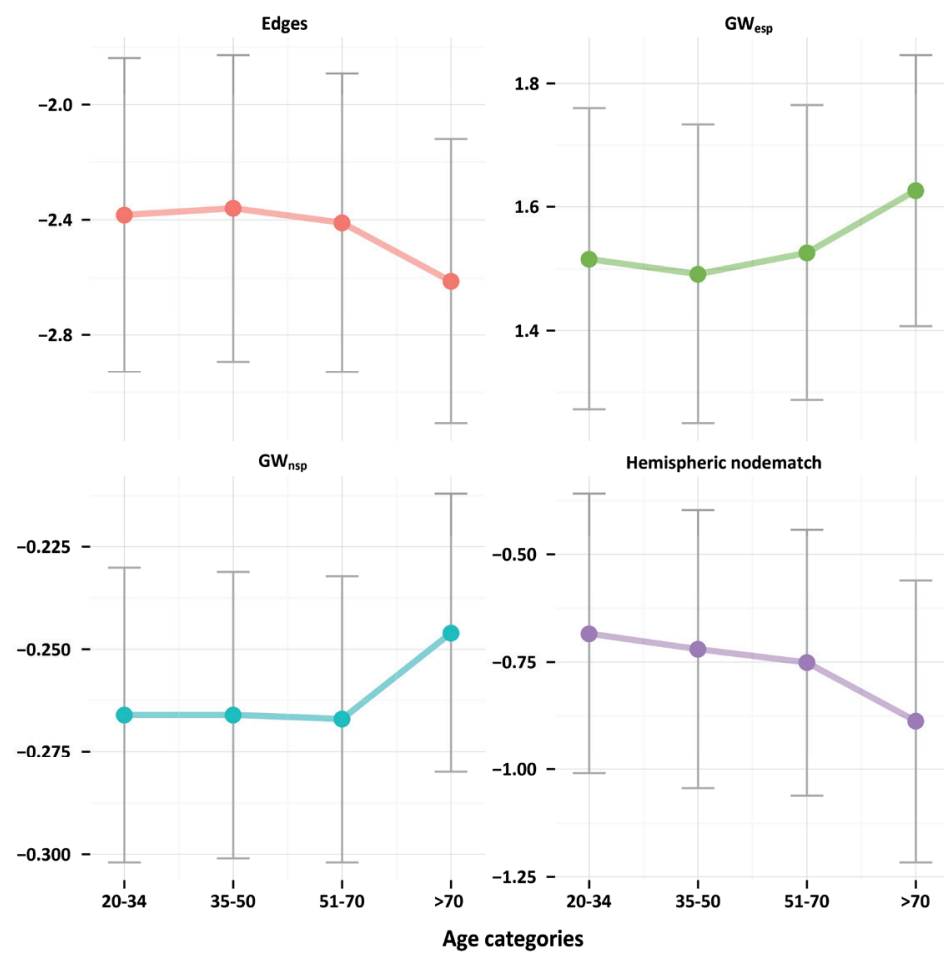

Figure 5. Network parameters across lifespan. Edges, $\mathrm{GW}_{\mathrm{ESP}}, \mathrm{GW}_{\mathrm{NSP}}$, and hemispheric nodematch for age categories $20-34,35-50,51-70$, and $>70$ years of age. 


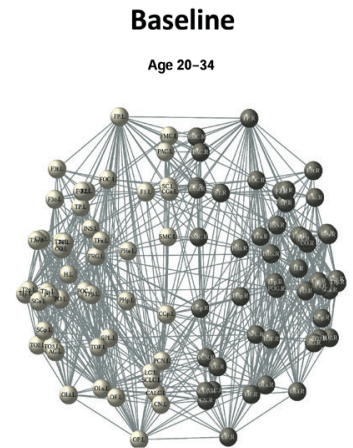

5\% Hub Damage

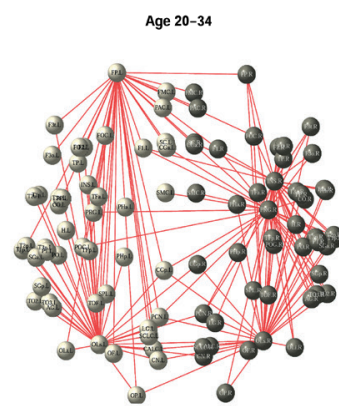

Age $35-50$

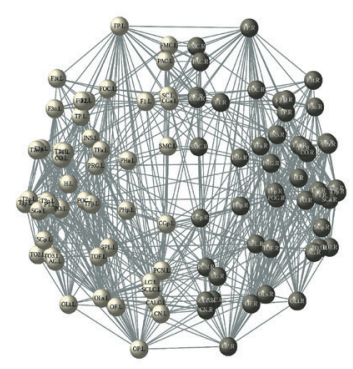

Age 51-70

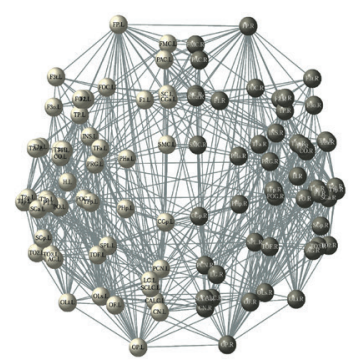

Age $>70$

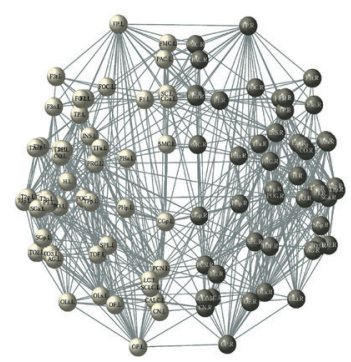

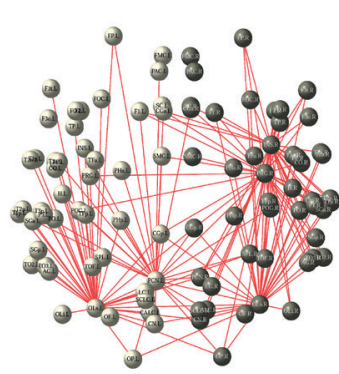

Age 51-70
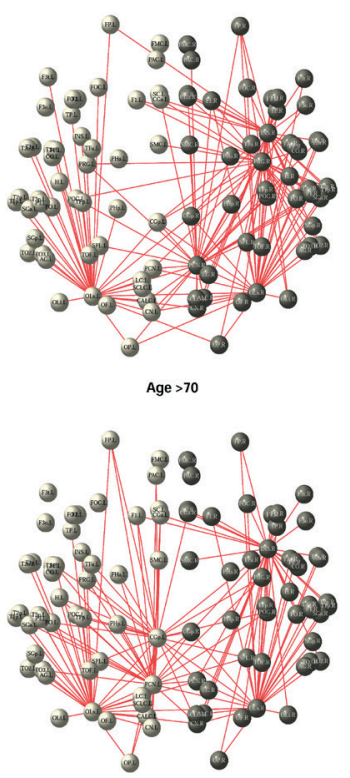

25\% Hub Damage

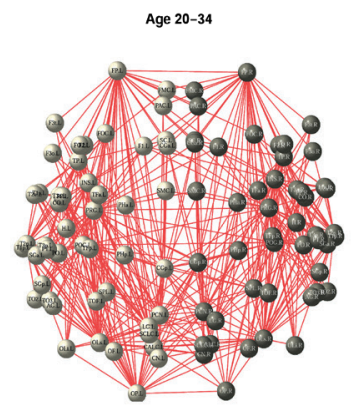

Age 35-50

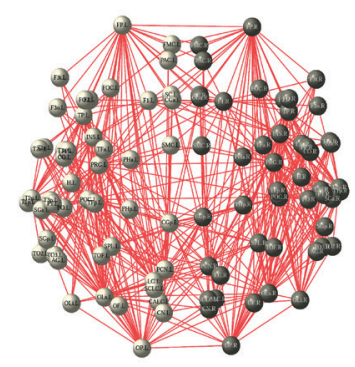

Age 51-70

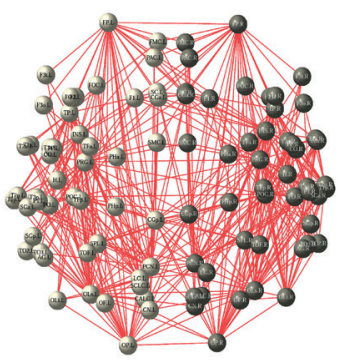

Age $>70$

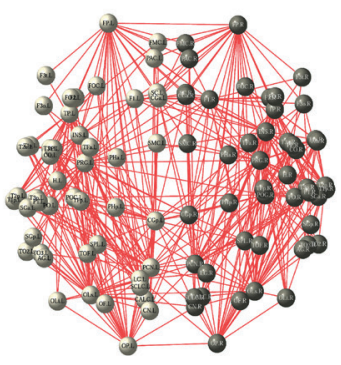

Figure 6. Baseline networks versus $\mathbf{5 \%}$ and $\mathbf{2 5 \%}$ hub damaged networks across age categories. The left column shows the average network for each age category. The middle and right columns show the lost edges (red lines) after randomly eliminating $5 \%$ and $25 \%$ of the hubs, respectively. 


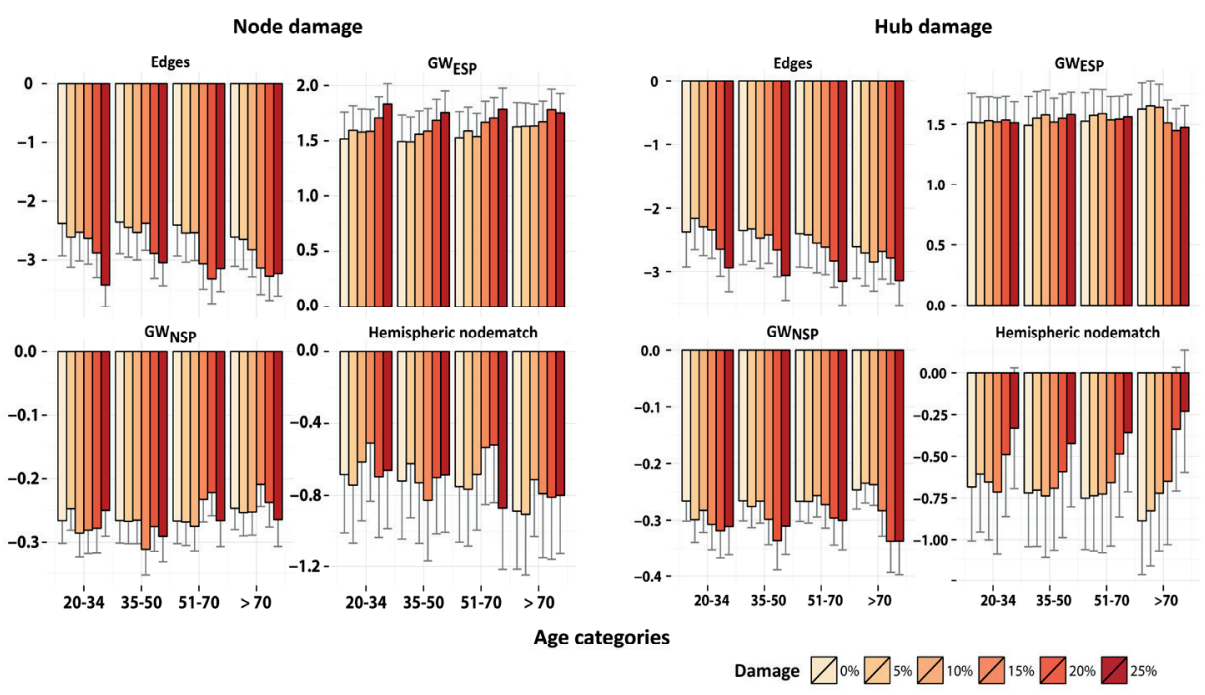

Figure 7. Effects of random-node and hub-node lesioning across age categories. Edge, GW $W_{E S P}, \mathrm{GW}_{\text {NSP }}$ and hemispheric nodematch plotted for age categories 20-34, 35-50, 51-70 and $>70$ years of age, at different percentages (color-coded) of simulated random-node (left) and hub-node (right) lesioning.

\section{Hub-node and random-node damage}

Effects of simulated hub-node lesions on network topology are visualized in Figure 6. Many connections, mostly in the temporal areas, were lost when $5 \%$ of hub nodes were randomly eliminated. Substantial global loss of connections was evident after elimination of $25 \%$ of hub-nodes, reflecting the abolishing effect of hub-damage on network density. Quantitative effects of simulated random-node and hub-node lesions, across age categories, are presented in Figure 7.

With random-node lesions the edges coefficient value decreased with increasing levels of lesioned nodes. A small increasing trend was detected for GW $\mathrm{GSP}_{\mathrm{ESP}}$. For GW $\mathrm{GSP}_{\text {NPP }}$ and hemispheric nodematch parameters, there was no consistent pattern in response to random-node eliminations.

Hub-node lesions resulted in reduced value of the edges coefficient, with a further decreasing effect as a function of age. $\mathrm{GW}_{\mathrm{ESP}}$ and $\mathrm{GW}_{\mathrm{NSP}}$ decreased, while hemispheric nodematch increased, after 15-25\% hub lesioning in the higher age categories.

Comparison of vulnerability to lesioning of hub-nodes across lifespan, quantified with Bayes Factors between age categories, is given in Table 3. Strongest differences between hub-lesion vulnerability across ages were found for local clustering and hemispheric nodematch. A change in $\mathrm{GW}_{\mathrm{ESP}}$, after $25 \%$ hub damage, was more likely in the age category $>70$ years than in the youngest age category. A change in hemispheric nodematch was more likely in the $>70$ years age group in comparison to the lowest age categories. 


\begin{tabular}{|c|c|c|c|}
\hline Substructure & $\begin{array}{c}\text { Age category } \\
\text { (baseline } \Delta 0 \%-25 \%)\end{array}$ & $\begin{array}{c}\text { Age category } \\
\text { (alternative } \Delta 0 \%-25 \%)\end{array}$ & $\log$ (Bayes Factor) \\
\hline edges & $20-34$ & $35-50$ & 2.7 \\
\hline edges & $20-34$ & $51-70$ & 2.3 \\
\hline edges & $20-34$ & $>70$ & 2.1 \\
\hline edges & $35-50$ & $51-70$ & -0.4 \\
\hline edges & $35-50$ & $>70$ & -0.6 \\
\hline edges & $51-70$ & $>70$ & -0.2 \\
\hline $\mathrm{GW}_{\mathrm{ESP}}$ & $20-34$ & $35-50$ & -0.2 \\
\hline $\mathrm{GW}_{\mathrm{ESP}}$ & $20-34$ & $51-70$ & 0.0 \\
\hline $\mathrm{GW}_{\mathrm{ESP}}$ & $20-34$ & $>70$ & -0.2 \\
\hline $\mathrm{GW}_{\mathrm{ESP}}$ & $35-50$ & $51-70$ & 0.2 \\
\hline $\mathrm{GW}_{\mathrm{ESP}}$ & $35-50$ & $>70$ & -0.1 \\
\hline $\mathrm{GW}_{\mathrm{ESP}}$ & $51-70$ & $>70$ & -0.3 \\
\hline $\mathrm{GW}_{\mathrm{NSP}}$ & $20-34$ & $35-50$ & 1.9 \\
\hline $\mathrm{GW}_{\mathrm{NSP}}$ & $20-34$ & $51-70$ & -1.7 \\
\hline $\mathrm{GW}_{\mathrm{NSP}}$ & $20-34$ & $>70$ & -0.4 \\
\hline $\mathrm{GW}_{\mathrm{NSP}}$ & $35-50$ & $51-70$ & -3.6 \\
\hline $\mathrm{GW}_{\mathrm{NSP}}$ & $35-50$ & $>70$ & -2.4 \\
\hline $\mathrm{GW}_{\mathrm{NSP}}$ & $51-70$ & $>70$ & 1.3 \\
\hline hemispheric nodematch & $20-34$ & $35-50$ & 5.1 \\
\hline hemispheric nodematch & $20-34$ & $51-70$ & 1.9 \\
\hline hemispheric nodematch & $20-34$ & $>70$ & 3.6 \\
\hline hemispheric nodematch & $35-50$ & $51-70$ & -3.2 \\
\hline hemispheric nodematch & $35-50$ & $>70$ & -1.5 \\
\hline hemispheric nodematch & $51-70$ & $>70$ & 1.7 \\
\hline
\end{tabular}

Table 3. Influence of simulated hub-damage on relative contributions of substructures (edges, $\mathrm{GW}_{\mathrm{ESP}}, \mathrm{GW}_{\mathrm{NSP}}$ and hemispheric nodematch) compared between age categories (20-34 years; 35-50 years; $51-70$ years; $>70$ years). The difference (delta) in the relative contribution of substructures between $0 \%$ and $25 \%$ hub lesioning within one age category (baseline) is compared with another age category (alternative) and expressed by the log (Bayes Factor).

\section{DISCUSSION}

We successfully applied Bayesian exponential random graph models on DTI-based structural brain networks over the human lifespan. The exponential random graph modeling approach (Robins et al., 2007b; Snijders et al., 2006) contrasts with conventional graph analysis in being generative instead of descriptive. Furthermore, exponential random graph models do not provide summaries of nodal and global network metrics, but show which local structures contribute to the global network topology and to what extent. Rather than providing solely summary statistics, exponential random graph models allow unbiased 
characterization of multiple network metric distributions and relational properties in the presence of significant changes in network densities (a problem of descriptive network studies in aging populations) (Caimo and Friel, 2011; Robins et al., 2007b; van Wijk et al., 2010).

In the present study we used a model with three local structures and one node attribute and we showed that simulations including these structures give representative (i.e. wellfitting) networks. The excellent goodness-of-fit of our limited number of local structures suggests that it is possible to describe a complex binary network with a small set of local parameters. We found that all included local structures contributed significantly to the global network topology across the lifespan. The values and signs of the parameters provide information about the relative contribution of local structures to the overall network topology (Robins et al., 2007b, 2007a). The negative coefficients for edges and global efficiency $\left(G_{N S P}\right)$ as well as the positive coefficient for local clustering ( $\left.G W_{E S P}\right)$ are in line with previous studies using the same approach in functional connectivity datasets (Simpson et al., 2011, 2012). The negative edges coefficient imply a lower expected edge density in comparison to a random graph with $50 \%$ edge probability, meaning that the brain network is relatively sparse, which is considered to be economical in terms of wiring costs (Achard and Bullmore, 2007; Bullmore and Sporns, 2012). The positive $\mathrm{GW}_{\mathrm{ESP}}$ reflects the many connections contributing to clustering in the brain. Clustering (and segregation) is a wellknown property of the brain contributing to local efficiency and segregation of brain function (Bullmore and Sporns, 2009; Rubinov and Sporns, 2010). The negative values for $\mathrm{GW}_{\text {NSP }}$ imply that non-connected nodes sharing one or multiple partners (i.e., indirectly connected) are also sparse. In this case we might think of long-range (within- and betweenhemisphere) connections that are important for the global efficiency (and integration) of brain function, which is an important feature of brain organization, also in terms of (a tradeoff between) wiring costs and efficiency in brain organization (Bullmore and Sporns, 2012). The negative hemispheric nodematch values indicate a tendency of nodes to make more connections within hemispheres than between hemispheres, which has been shown by many connectivity studies.

Statistical analyses of exponential random graph models across the lifespan revealed a relatively stable contribution of network properties (i.e. local clustering and global efficiency) over all age categories, in contrast with previous literature showing alterations in both structural (Dennis et al., 2013; Gong et al., 2009; Hagmann et al., 2010; Montembeault et al., 2012; Otte et al., 2015; Wu et al., 2012; Zhu et al., 2012) and functional brain networks across life-span (Achard and Bullmore, 2007; Betzel et al., 2014; Meier et al., 2012; Meunier et al., 2009; Simpson and Laurienti, 2015b; Smit et al., 2016; Spreng and Schacter, 2012; Wang et al., 2012). Furthermore, our findings are also in contrast with significant differences found in a previous exponential random graph modeling study in 
functional networks (Simpson et al., 2011), and a recently developed similar approach (also discussed below) which revealed differences in functional networks across the lifespan, such as older adults having stronger connections between highly clustered nodes, or less assortativity in visual and multisensory regions (Simpson and Laurienti, 2015b). We speculate that functional networks might be more prone to changes across the lifespan, while structural networks remain quite stable, when taking into account density differences and mutual dependencies of network properties.

Simulation of random and hub lesions showed minimal effects on the contribution of local structures within age categories. This emphasizes the relative robustness to (simulated) brain network damage of local network structures, i.e. unless brain damage local network features that give rise to the global network topology, such as local clustering and global efficiency, are not significantly affected. Nevertheless, hub-node lesioning had a stronger effect in people above 70 years of age as compared to the youngest age category (i.e. $\log \left(\right.$ Bayes Factor) $>3$ ). We found trends of increasing local clustering $\left(\mathrm{GW}_{\mathrm{ESP}}\right)$ with more random-node eliminations up to 70 years of age, after which $\mathrm{GW}_{\mathrm{ESP}}$ remained the same or tended to decrease with increasing hub damage. Hub-node lesioning resulted in lower $\mathrm{GW}_{\text {NSP }}$ and higher hemispheric nodematches. These two trends increased with higher age, which may indicate that older people are more vulnerable to hub-node damage (possibly associated with degenerative disease such as Alzheimer's disease (Crossley et al., 2014)) than younger people. This may be due to the overall decrease in network density across age, perhaps in combination with increased vulnerability due to high intrinsic neuronal activity of hub node regions (de Haan et al., 2012). Our findings are in line with previous simulation studies in human and animals, which have shown that computational lesioning of hub regions have stronger effects on structural and functional network topology than lesioning of random nodes (Alstott et al., 2009; Crossley et al., 2014; Kaiser et al., 2007).

There are several other approaches, which are of interest in light of exponential random graph modeling that has been used to capture generative network information. One such approach is based on counting motifs (Sporns and Kötter, 2004), which needs post-hoc statistical assessment, while statistical testing is inherent for exponential random graph models. However, motif counting and corresponding statistical results can provide good starting conditions for choosing appropriate exponential random graph models (van Wijk et al., 2010). In particular because earlier problems such as the intractability of the normalizing constant and model degeneracy have been solved (Caimo and Friel, 2011; Handcock, 2003). Other interesting approaches (complementary with exponential random graph modeling) are mixed models (Simpson et al., 2014) and Gibbs distribution models (La Rosa et al., 2016). In contrast to mixed models, exponential random graph models are limited in examining specific connections and investigating associations between network and phenotypes, and less suited for group comparisons, but are able to capture the inherent dependence 
structure of complex networks (Simpson et al., 2014). Recently, mixed models were adapted to two-part mixed-effects modeling, to account for the dependence structure (Simpson and Laurienti, 2015a, 2015b). In contrast to Gibbs distribution models (i.e. modeling of graph populations), both exponential random graph models and mixed models ignore overall network differences, but are able to capture topological features (La Rosa et al., 2016). It would be interesting to adapt and integrate modeling approaches, and if impossible, it is recommended to consider the simultaneous use of complementary approaches in future research.

In line with the earlier mentioned growth models (Betzel et al., 2015; Vertes et al., 2012), exponential random graph models aim for a parsimonious description of the human brain network. Comparison of different generative models with several parameters provided the best model or optimal parameters, resulting in the generation of synthetic networks with very similar topological characteristics as real brain networks (Betzel et al., 2015; Vertes et al., 2012). These studies showed that cost minimization alone does not explain the global network topology, but that the models require information on the pairwise similarity of nodes (i.e. homophily; e.g. similar function or similar degree) to accurately simulate the brain network (Betzel et al., 2015; Vertes et al., 2012). Although modeling a geometrically preferential attachment parameter (i.e. based on distance) is possible with exponential random graph modeling, it requires distance information on all possible node pairs (which is difficult to determine with sparse connectivity data; but Euclidean distance approximations could be used as proxies, with good results). Our study shows that node attributes (e.g. locational information like hemisphere) can be easily implemented in the exponential random graph modeling approach (Caimo and Friel, 2014). It would therefore be interesting to include other attributes in future research (e.g. sensory, motor and association areas) to test for assortativity (i.e. homophily) in terms of functional or organizational similarity (Betzel et al., 2015; Vertes et al., 2012). As such it would also be possible to simulate and compare different models to examine which parameters are of importance to the global network topology.

Recently, another approach, minimum spanning trees, has been proposed to circumvent the methodological issues that arise in the comparison of networks that differ in size and density (Stam et al., 2014). The tree describes the backbone of a network, which contains only the strongest connections, thereby facilitating comparability of different network topologies without aforementioned normalization and correction steps. Several studies have shown the usefulness of the minimum spanning tree approach by detecting subtle conditional and developmental network changes, both between and within studies (Stam et al., 2014). To date, no comparative studies between minimum spanning trees and generative network modeling are known to us. Minimum spanning trees are potentially relevant for modeling of very large (edge-weighted) networks, as exponential random graph 
models are computationally much more demanding. A drawback of exponential random graph models is the nonlinear increase in computational load with increasing network size and model complexity. Furthermore implementation for edge-valued networks (Desmarais and Cranmer, 2012) are not yet available in the Bayesian framework.

One limitation of the present study might be that subcortical regions were not included. However, the accuracy of registration and alignment of sub-cortical regions is difficult if compared with cortical regions, even when nonlinear registration procedures are used, and might thus give unreliable networks. Another limitation might be the construction of representative group-networks based on a certain, but arbitrary, edge prevalence threshold. Future studies might look into different approaches to fit group networks, e.g. based on exponential random graph modeling (Simpson et al., 2012) or a fully Bayesian description of networks (Hinne et al., 2012; Janssen et al., 2014). The present study did not investigate whether exponential random graph models can solve the problems with differences in network size. This highly interesting and relevant question should therefore be addressed in future research.

Future studies may explore Bayesian generative modeling in other network datasets in health and disease, and extend the model with more substructures and attributes (e.g. cortical regions, assortative connections, brain location, interactions), to assess the effect of different network properties between brain regions (Dennis et al., 2013) as well as constraining influences of network topology and connections (Bullmore and Sporns, 2012; Kaiser and Hilgetag, 2006). 


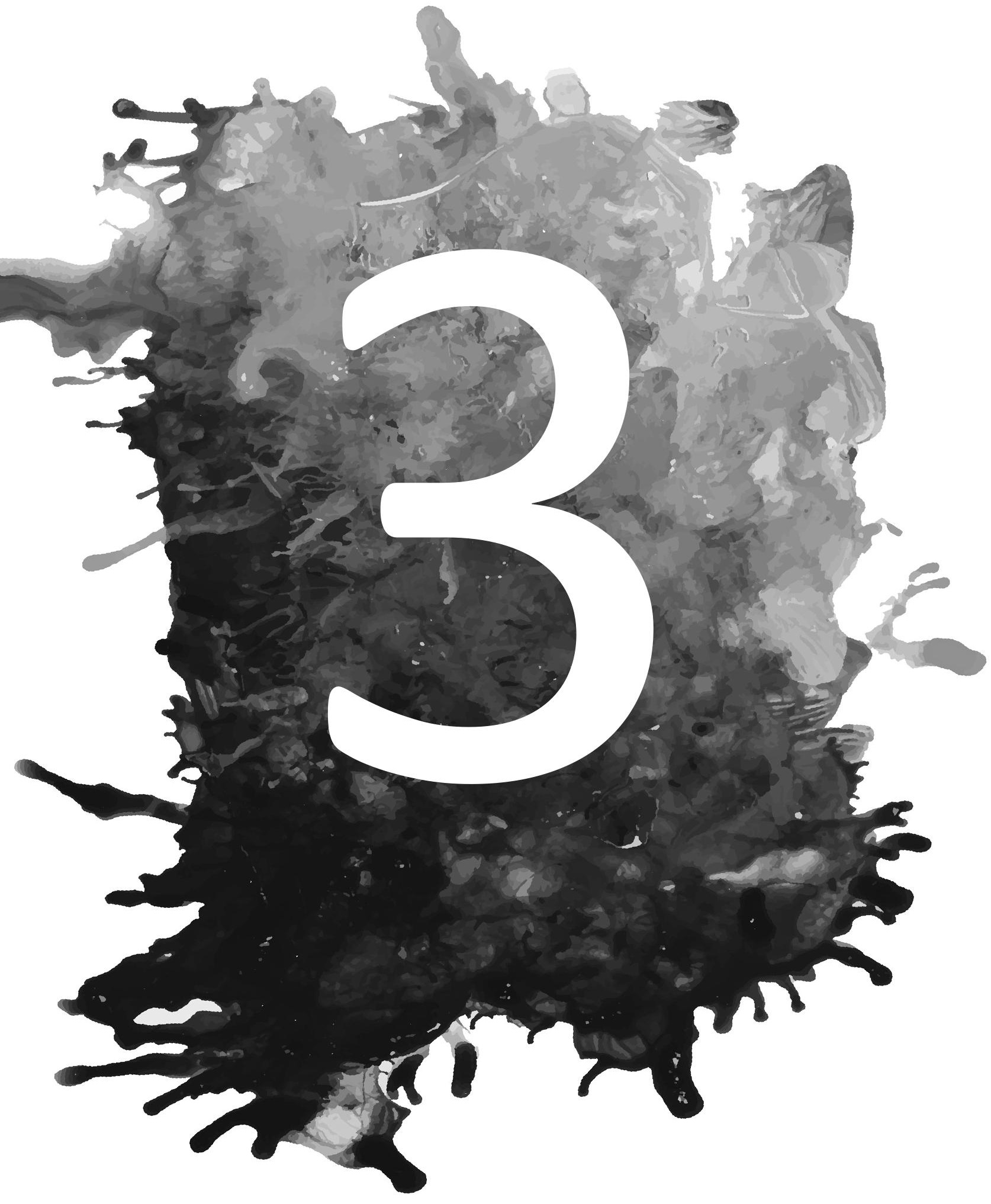




\section{Chapter 3}

\section{Diffusion MRI-based Cortical Connectome Reconstruction - \\ Dependency on Tractography Procedures and Neuroanatomical Characteristics}

M.R.T. Sinke, W.M. Otte, D. Christiaens, O. Schmitt, A. Leemans, A. van der Toorn, R.A. Sarabdjitsingh, M. Joëls \& R.M. Dijkhuizen 


\begin{abstract}
Diffusion MRI (dMRI)-based tractography offers unique abilities to map whole-brain structural connections in human and animal brains. However, dMRI-based tractography indirectly measures white matter tracts, with suboptimal accuracy and reliability. Recently, sophisticated methods including constrained spherical deconvolution (CSD) and global tractography have been developed to improve tract reconstructions through modeling of more complex fiber orientations. Our study aimed to determine the accuracy of connectome reconstruction for three dMRI-based tractography approaches: diffusion tensor (DT)-based, CSD-based and global tractography. Therefore, we validated whole brain structural connectome reconstructions based on ten ultrahigh-resolution dMRI rat brain scans and 106 cortical regions, from which varying tractography parameters were compared against standardized neuronal tracer data. All tested tractography methods generated considerable numbers of false positive and false negative connections. There was a parameter range trade-off between sensitivity: 0.06-0.63 interhemispherically and 0.220.86 intrahemispherically; and specificity: 0.99-0.60 interhemispherically and 0.99-0.23 intrahemispherically. Furthermore, performance of all tractography methods decreased with increasing spatial distance between connected regions. Similar patterns and trade-offs were found when we applied spherical deconvolution informed filtering of tractograms (SIFT), streamline thresholding and group-based average network thresholding. Despite the potential of CSD-based and global tractography to handle complex fiber orientations at voxel level, reconstruction accuracy, especially for long-distance connections, remains a challenge. Hence, connectome reconstruction benefits from varying parameter settings and combination of tractography methods to account for anatomical variation of neuronal pathways.
\end{abstract}




\section{INTRODUCTION}

Mapping brain-wide structural connectivity has become an important goal in neuroscience and is the primary focus of the field of connectomics (Sporns, 2010; Sporns et al., 2005). This field aims to construct comprehensive maps of all neuronal elements and their connections in different organisms, including a wide range of mammalian species. Connectomes can provide crucial insights into brain functioning in health and disease as (changes in) structural connections underlie at least part of functional and behavioral phenotypes (Bassett and Bullmore, 2009; Sporns, 2010; Sporns et al., 2005; Stam, 2014).

Unraveling the human connectome is a great challenge (Poldrack and Farah, 2015) and its reconstruction would be a fundamental breakthrough in neuroscience (Sporns, 2010; Sporns et al., 2005). So far, a complete connectome has been mapped only for $C$. elegans (White et al., 1986). Accurate connectome reconstructions at micro-, meso- and macroscale in other species, including Drosophila (Chiang et al., 2011), mouse (Oh et al., 2014), rat (Schmitt and Eipert, 2012), macaque (Stephan et al., 2001) and human (van Essen et al., 2012) involve ongoing efforts in large-scale multicenter projects, such as the Human Connectome Project (van Essen et al., 2012), the BRAIN project (NIH, 2014), the Brainnetome Project (Jiang, 2013) and the CONNECT project (Assaf et al., 2013).

Diffusion-weighted tractography has offered exclusive means to map structural connections non-invasively in animal and human brain, and is considered a very useful and powerful technique to unravel the human connectome at meso- and macro-level (Basser et al., 1994a; Le Bihan et al., 1986; Jbabdi and Johansen-Berg, 2011; Jones, 2008; Mori et al., 1999). However, the anatomical accuracy of diffusion-weighted tractography, which infers neuronal pathways from tissue water diffusion direction, continues to be a matter of controversy, and reconstructing connectomes with both high sensitivity and high specificity remains challenging (Azadbakht et al., 2015; Bastiani et al., 2012; Calabrese et al., 2015; Donahue et al., 2016; Jbabdi et al., 2013, 2015; Knösche et al., 2015; Reveley et al., 2015; Sporns et al., 2005; Thomas et al., 2014). This may lead to biased characterization of connectome topology, particularly in the presence of false positive tract reconstructions (Drakesmith et al., 2015; Zalesky et al., 2016).

The classical approach of diffusion tensor (DT)-based deterministic tractography (Basser et al., 2000) uses the diffusion tensor model (i.e. estimating one principal direction in each voxel) to reconstruct fiber tracts in the brain. This method is particularly hampered by voxels containing multiple fiber orientations such as crossing, bending or fanning fibers (Jbabdi and Johansen-Berg, 2011). At standard spatial resolutions around a few $\mathrm{mm}$, multiple fiber orientations affect the diffusivity patterns in up to $90 \%$ of white matter voxels in the human brain (Jeurissen et al., 2013). In order to improve the characterization of the intravoxel diffusion profile and the accuracy of subsequent tractography, high-angular resolution diffusion imaging (HARDI) methods have been developed (Descoteaux et al., 2007; Jeurissen 
et al., 2009; Tax et al., 2014; Tournier et al., 2011). For instance, constrained spherical deconvolution (CSD), developed by Tournier and colleagues, models the diffusion profile in voxels with multiple fiber orientations (Tournier et al., 2004, 2007). Instead of estimating one principal fiber direction, CSD estimates multiple fiber directions within a voxel, resulting in a fiber orientation distribution (FOD) that may have multiple peaks (e.g. four in case of two crossing fibers in one voxel) (Tournier et al., 2010, 2012). CSD-based tractography uses $2^{\text {nd }}$ order integration over fiber orientation distributions, thereby including information from orientations in neighboring voxels (Tournier et al., 2010, 2012). This solves some of the typical tractography errors, but false positive tract reconstructions may still arise from noisy (spurious) peaks or from ambiguous fanning and bending fiber populations (Jbabdi and Johansen-Berg, 2011). Recently a new method, called Spherical-deconvolution Informed Filtering of Tractograms (SIFT) has been proposed to reduce local streamline density bias by filtering the tractogram (i.e. selectively removing streamlines) to improve the fit between the overall distribution of streamlines and the FOD in every voxel, estimated from the diffusion-weighted image, which may improve accuracy of connectome reconstruction (Smith et al., 2013, 2015a). Still, additional errors may arise from the conventional method of reconstructing tracts from streamlines that are propagated in a step-wise approach. Consequently, long-distance connections are more difficult to track as the reconstruction error accumulates with each tracking step (Donahue et al., 2016; Jbabdi et al., 2015; Reveley et al., 2015; Zalesky and Fornito, 2009). Instead of tracking streamlines, alternative tractography approaches model tracts at a global rather than voxel level, to solve local reconstruction issues with bending and fanning fiber populations as well as to mitigate the reconstruction error accumulation at longer distances. Global tractography aims to find the full track (i.e. whole-brain) configuration that most optimally fits the acquired data (Christiaens et al., 2015a; Mangin et al., 2013; Reisert et al., 2011). Modern methods such as CSD-based tractography, global tractography and SIFT may significantly improve the performance of brain-wide tractography and contribute to more accurate connectome reconstructions. However, anatomical validation is largely lacking.

Validation studies in mammalian species from which neuronal tracing data is available, offer powerful means to directly compare diffusion-based tract reconstructions against true neuroanatomical connectivity (Calabrese et al., 2015; Chen et al., 2015; Dauguet et al., 2007; Dyrby et al., 2007; Gao et al., 2013; Thomas et al., 2014). This approach has been successfully applied to validate tractography methods and/or to verify reconstruction of specific white matter structures in squirrel monkeys (Gao et al., 2013), mini-pigs (Dyrby et al., 2007) and macaques (Reveley et al., 2015; Thomas et al., 2014). However, validation of whole-brain connectome reconstruction (i.e. structural connectivity between multiple brain regions) from contemporary diffusion-based tractography approaches is currently lacking. Therefore we compared brain-wide structural connectome reconstruction from DT-based, 
CSD-based and global tractography, using ultrahigh-resolution diffusion-weighted images of rat brain, against neuroanatomical connectivity measures from tracer studies. We evaluated tractography results across different anatomical sites and distances in the brain and determined how parameter settings affect the connectome reconstruction. In addition, we assessed the effect of SIFT, streamline thresholding and group-based average network thresholding on connectome reconstructions.

\section{METHODS}

\section{Animal preparation}

All animal procedures were approved by the Animal Experiments Committee of the University Medical Center Utrecht and Utrecht University, and experiments were performed in accordance with the guidelines of the European Communities Council Directive. Ten healthy adult (12-13 weeks old) male Wistar rats that were randomly distributed for an earlier study (Dijkhuizen et al., 2017) were group-housed under standard conditions with light/darks cycle on/off from 07:00 till 19:00. Animals were sacrificed in the morning between 09:00 and 12:00 by intraperitoneal injection of an overdose of pentobarbital, followed by transcardial perfusion-fixation with $4 \%$ paraformaldehyde in phosphate-buffered saline. Brains inside the intact skull were extracted and positioned in a container filled with proton-free oil (Fomblin ${ }^{\circledR}$ ) for MRI scanning.

\section{Diffusion MRI}

Diffusion-weighted imaging was performed with a 9.4T small-animal MR scanner (Varian, Palo Alto, CA, USA) and a custom-made solenoid coil with an internal diameter of $2.6 \mathrm{~cm}$. High spatial and angular resolution diffusion imaging (HARDI) was accomplished using an 8shot 3D echo planar imaging (EPI) sequence, with repetition time $(T R)=500 \mathrm{~ms}$, echo time $(T E)=32.4 \mathrm{~ms}, \Delta / \delta 15 / 4 \mathrm{~ms}$ and $\mathrm{b}$-value $=3842 \mathrm{~s} / \mathrm{mm}^{2}$. The field-of-view (FOV) was $19.2 \mathrm{x}$ $16 \times 33 \mathrm{~mm}^{3}$ and the data matrix size was $128 \times 108 \times 220$, resulting in a voxel resolution of $150 \times 148 \times 150 \mu^{3}$. The HARDI protocol consisted of acquisition of 5 non-diffusionweighted $\left(b_{0}\right)$ images and 60 images with different non-collinear diffusion-weighting directions on a half sphere, with four averages per image. Total acquisition time was 8 hours per sample.

\section{Data processing}

Preprocessing was done with FSL (http://www.fmrib.ox.ac.uk/fsl/) (Jenkinson et al., 2012) and included brain extraction (Smith, 2002) and registration steps. Correction for eddy currents or bias field was not necessary because we used a special volume coil and MRI pulse sequence (with extra delays after sinus-shaped gradients) that allowed homogenous imaging of post mortem brain tissue. With FLIRT (Jenkinson and Smith, 2001; Jenkinson et 
al., 2002) and FNIRT (Andersson et al., 2007), diffusion-weighted images of individual rat brains were linearly and non-rigidly aligned to a rat brain template matched with a custombuilt 3D model (Majka et al., 2012) of the $5^{\text {th }}$ edition of the Paxinos and Watson rat brain atlas (Paxinos and Watson, 2005). All 106 bilateral atlas regions that cover the entire cortex were subsequently back-projected into subject space for subsequent tractography.

\section{Diffusion-based tractography}

All diffusion-based tractography approaches and subsequent connectome reconstructions were performed in MRtrix $3^{\circledR}$ (http://www.mrtrix.org/) (Tournier et al., 2012).

\section{DT-based tractography}

The DT model was directly estimated from the raw diffusion weighted data using standard log-linear least-squares regression (Basser et al., 1994b). Fractional anisotropy (FA) maps were determined from the diffusion tensor. Streamlines were generated from randomly placed seeds over a mask covering the whole brain. We varied the following parameters for streamline computation: step size $(15 \mu \mathrm{m}, 30 \mu \mathrm{m}$ and $50 \mu \mathrm{m})$, FA threshold $(0.05,0.10,0.15$ and 0.20) (streamlines will terminate in voxels with a sub-threshold FA value), angular threshold $\left(20-80^{\circ}\right.$ with steps of $\left.10^{\circ}\right)$ (streamlines will terminate when bending with an angle exceeding the threshold), and number of streamlines $\left(25,50,100\right.$ and $\left.250 \times 10^{3}\right)$.

\section{CSD-based tractography}

The CSD-model was computed from the signal amplitude yielding FOD maps used for CSDbased tractography. Streamlines were generated from randomly placed seeds over a brain mask. We varied the following tractography parameters: step size $(50 \mu \mathrm{m}, 75 \mu \mathrm{m}$ and 100 $\mu \mathrm{m})$, angular threshold $\left(20-80^{\circ}\right.$ with steps of $\left.10^{\circ}\right)$, number of streamlines $(25,50,100$ and $\left.250 \times 10^{3}\right)$, and FOD amplitude threshold $(0.1,0.125,0.15,0.175)$. Higher FOD amplitude thresholds will cause a more rigid delineation of tracts since streamlines will not follow directions with low FOD amplitudes, whereas lower thresholds will lead to more variation in tract reconstructions.

\section{Global tractography}

Global tractography uses the estimation of distinct diffusion profiles (i.e. response functions) for different tissue classes (i.e. white matter, gray matter and cerebrospinal fluid) to deal with partial volume effects. Because the postmortem rat brains in our study contained relatively little cerebrospinal fluid and were embedded in proton-free oil, we restricted tissue classification to gray and white matter. In global tractography, tracts are built from segments, called particles (default length was $100 \mu \mathrm{m}$ for our dataset). In line with a previous validation study in human brain (Christiaens et al., 2015a), we varied two key parameters, i.e. particle potential $(0.1,0.2,0.5,1,2$ and 5$)$ and connection potential 
$(0.01,0.02,0.05,0.1,0.2$ and 0.5$)$. The particle potential regulates the number and distribution of particles, where higher values weigh towards larger white matter structures (i.e. less particles in cortical areas), whereas lower values increase the number of particles in structures like the cortex. The connection potential drives the connection of these segments (i.e. particles) and thereby the formations of tracts, where higher values increase the formation of reconstructed tracts.

\section{Spherical deconvolution Informed Filtering of Tractograms (SIFT)}

We applied SIFT (Smith et al., 2013, 2015a) on DT-based and CSD-based tractograms, which optimizes and fits the reconstructed white matter tracts (i.e. streamlines) to the underlying diffusion-weighted images. This approach normalizes and corrects the number of streamlines (i.e. filters the tractogram) based on spherical deconvolution of the diffusion signal. This leads to a data-driven decrease in network density, without requirement of an arbitrary threshold (such as a streamline threshold). We evaluated the effect of SIFT correction compared to standard CSD-based and DT-based tractography for 250,000 streamlines (since a high number of streamlines is required for SIFT). We limited our analysis to the default step sizes for DT- and CSD-based tractography (respectively $15 \mu \mathrm{m}$ and 75 $\mu \mathrm{m})$, two FOD/FA thresholds and four angle thresholds, to manage computational time.

\section{Diffusion tractography-based connectome reconstruction}

Connectomes were constructed by comparing, for each brain, generated tracks between 106 cortical regions from the Paxinos and Watson rat brain atlas (Table 1). Two regions were considered to be structurally connected if one or more streamlines had their endpoints in both regions. The number of streamlines connecting pairs of regions was ignored; i.e. all connections were binarized.

\section{Neuroanatomical tracing database}

Neuronal tracer data were extracted from the rat connectome database (http://neuroviisas.med.uni-rostock.de/connectome/index.php) that was built with neuroVIISAS (Schmitt and Eipert, 2012). This database contains more than 450,000 ipsi- and 175,650 contralateral connections from more than 6,183 peer reviewed publications which describe connectivity observations based on anterograde and retrograde neuronal tracer injections in normal juvenile or adult rats. This tracer-based connectome covers all cortical atlas regions. We extracted connectivity information for the same 106 cortical regions (Table 1) as used for diffusion-based connectome reconstructions. Several tracer-based connections (between pairs of regions) are unidirectional. We considered two regions neuroanatomically connected irrespective of directionality. As such, the tracer-based connectome had a network density (i.e. the proportion of true connections out of all potential connections) of 0.27 (Figure S1). 


\begin{tabular}{|c|c|c|}
\hline \multicolumn{3}{|c|}{ Cortical regions used for connectome construction } \\
\hline Number & ROI-label & Anatomical region \\
\hline 1 & le_AID & Agranular insular cortex dorsal part left \\
\hline 2 & ri_AID & Agranular insular cortex dorsal part right \\
\hline 3 & le_AIP & Agranular insular cortex posterior part left \\
\hline 4 & ri_AIP & Agranular insular cortex posterior part right \\
\hline 5 & le_AIV & Agranular insular cortex ventral part left \\
\hline 6 & ri_AIV & Agranular insular cortex ventral part right \\
\hline 7 & le_Au1 & Primary auditory cortex left \\
\hline 8 & ri_Au1 & Primary auditory cortex right \\
\hline 9 & le_AuD & Secondary auditory cortex dorsal area left \\
\hline 10 & ri_AuD & Secondary auditory cortex dorsal area right \\
\hline 11 & le_AuV & Secondary auditory cortex ventral area left \\
\hline 12 & ri_AuV & Secondary auditory cortex ventral area right \\
\hline 13 & le_cg1 & Cingulate cortex area 1 left \\
\hline 14 & ri_Cg1 & Cingulate cortex area 1 right \\
\hline 15 & le_Cg2 & Cingulate cortex area 2 left \\
\hline 16 & ri_Cg2 & Cingulate cortex area 2 right \\
\hline 17 & le_DI & Dysgranular insular cortex left \\
\hline 18 & ri_DI & Dysgranular insular cortex right \\
\hline 19 & le_DIEnt & Dorsal intermediate entorhinal cortex \\
\hline 20 & ri_DIEnt & Dorsal intermediate entorhinal cortex \\
\hline 21 & le_DLEnt & Dorsolateral entorhinal cortex left \\
\hline 22 & ri_DLEnt & Dorsolateral entorhinal cortex right \\
\hline 23 & le_DLO & Dorsolateral orbital cortex left \\
\hline 24 & ri_DLO & Dorsolateral orbital cortex right \\
\hline 25 & le_Ect & Ectorhinal cortex left \\
\hline 26 & ri_Ect & Ectorhinal cortex right \\
\hline 27 & le_Fr3 & Frontal cortex area 3 left \\
\hline 28 & ri_Fr3 & Frontal cortex area 3 right \\
\hline 29 & le_FrA & Frontal association cortex left \\
\hline 30 & ri_FrA & Frontal association cortex right \\
\hline 31 & le_Gl & Granular insular cortex left \\
\hline 32 & ri_GI & Granular insular cortex right \\
\hline 33 & le_IL & Infralimbic cortex left \\
\hline 34 & ri_IL & Infralimbic cortex right \\
\hline 35 & le_LEnt & Lateral entorhinal cortex left \\
\hline 36 & ri_LEnt & Lateral entorhinal cortex right \\
\hline 37 & le_LO & Lateral orbital cortex left \\
\hline 38 & ri_LO & Lateral orbital cortex right \\
\hline 39 & le_LPtA & Lateral parietal association cortex left \\
\hline 40 & ri_LPtA & Lateral parietal association cortex right \\
\hline 41 & le_M1 & Lateral agranular prefrontal cortex left \\
\hline 42 & ri_M1 & Lateral agranular prefrontal cortex right \\
\hline 43 & le_M2 & Medial agranular prefrontal cortex left \\
\hline 44 & ri_M2 & Medial agranular prefrontal cortex right \\
\hline 45 & le_MO & Medial orbital cortex left \\
\hline 46 & ri_MO & Medial orbital cortex right \\
\hline 47 & le_mPFC & Medial prefrontal cortex left \\
\hline 48 & ri_mPFC & Medial prefrontal cortex right \\
\hline 49 & le_MPtA & Medial parietal association cortex left \\
\hline 50 & ri_MPtA & Medial parietal association cortex right \\
\hline 51 & le_PRh & Perirhinal cortex left \\
\hline 52 & ri_PRh & Perirhinal cortex right \\
\hline 53 & le_PtPC & Parietal cortex posterior area caudal part left \\
\hline 54 & ri_PtPC & Parietal cortex posterior area caudal part right \\
\hline 55 & le_PtPD & Parietal cortex posterior area dorsal part left \\
\hline
\end{tabular}




\begin{tabular}{|c|c|c|}
\hline Number & ROI-label & Anatomical region \\
\hline 56 & ri_PtPD & Parietal cortex posterior area dorsal part right \\
\hline 57 & le_PtPR & Parietal cortex posterior area rostral part left \\
\hline 58 & ri_PtPR & Parietal cortex posterior area rostral part right \\
\hline 59 & le_RSD & Retrosplenial dorsal left \\
\hline 60 & ri_RSD & Retrosplenial dorsal right \\
\hline 61 & le_RSGa & Retrosplenial granular cortex a region left \\
\hline 62 & ri_RSGa & Retrosplenial granular cortex a region right \\
\hline 63 & le_RSGb & Retrosplenial granular cortex $\mathrm{b}$ region left \\
\hline 64 & ri_RSGb & Retrosplenial granular cortex $\mathrm{b}$ region right \\
\hline 65 & le_RSGc & Retrosplenial granular cortex $\mathrm{c}$ region left \\
\hline 66 & ri_RSGc & Retrosplenial granular cortex $\mathrm{c}$ region right \\
\hline 67 & le_S1 & Primary somatosensory cortex left \\
\hline 68 & ri_S1 & Primary somatosensory cortex right \\
\hline 69 & le_S1BFa & Primary somatosensory cortex barrel field left \\
\hline 70 & ri_S1BFa & Primary somatosensory cortex barrel field right \\
\hline 71 & le_S1DZ & Primary somatosensory cortex dysgranular region left \\
\hline 72 & ri_S1DZ & Primary somatosensory cortex dysgranular region right \\
\hline 73 & le_S1DZO & Primary somatosensory cortex oral dysgranular region left \\
\hline 74 & ri_S1Dzo & Primary somatosensory cortex oral dysgranular region right \\
\hline 75 & le_S1FL & Primary somatosensory cortex forelimb region left \\
\hline 76 & ri_S1FL & Primary somatosensory cortex forelimb region right \\
\hline 77 & le_s1HL & Primary somatosensory cortex hindlimb region left \\
\hline 78 & ri_S1HL & Primary somatosensory cortex hindlimb region right \\
\hline 79 & le_s1s & Primary somatosensory cortex jaw region left \\
\hline 80 & ri_s1J & Primary somatosensory cortex jaw region right \\
\hline 81 & le_s1Sh & Primary somatosensory cortex shoulder region left \\
\hline 82 & ri_S1Sh & Primary somatosensory cortex shoulder region right \\
\hline 83 & le_S1Tr & Primary somatosensory cortex trunk region left \\
\hline 84 & ri_s1Tr & Primary somatosensory cortex trunk region right \\
\hline 85 & le_S1ULp & Primary somatosensory cortex upper lip region left \\
\hline 86 & ri_S1ULp & Primary somatosensory cortex upper lip region right \\
\hline 87 & le_s2 & Secondary somatosensory cortex left \\
\hline 88 & ri_s2 & Secondary somatosensory cortex right \\
\hline 89 & le_TeA & Temporal association cortex 1 left \\
\hline 90 & ri_TeA & Temporal association cortex 1 right \\
\hline 91 & le_v1 & Primary visual cortex left \\
\hline 92 & ri_V1 & Primary visual cortex right \\
\hline 93 & le_v1B & Primary visual cortex binocular area left \\
\hline 94 & ri_V1B & Primary visual cortex binocular area right \\
\hline 95 & le_v1M & Primary visual cortex monocular area left \\
\hline 96 & ri_v1M & Primary visual cortex monocular area right \\
\hline 97 & le_V2L & Secondary visual cortex lateral area left \\
\hline 98 & ri_V2L & Secondary visual cortex lateral area right \\
\hline 99 & le_V2ML & Secondary visual cortex medial area left \\
\hline 100 & ri_V2ML & Secondary visual cortex medial area right \\
\hline 101 & le_V2MM & Secondary visual cortex mediomedial area left \\
\hline 102 & ri_V2MM & Secondary visual cortex mediomedial area right \\
\hline 103 & le_VIEnt & Ventral intermediate entorhinal cortex left \\
\hline 104 & ri_VIEnt & Ventral intermediate entorhinal cortex right \\
\hline 105 & le_vo & Ventral orbital cortex left \\
\hline 106 & ri_vo & Ventral orbital cortex right \\
\hline
\end{tabular}

Table 1. Atlas-based network regions. For all included cortical regions $(\mathrm{N}=106)$ the Paxinos and Watson atlas region of interest (ROI) label and full anatomical names are shown. 


\section{Quality of diffusion tractography-based reconstructions}

Sensitivity, specificity and Jaccard index

The diffusion tractography-based connectivity (i.e. structural connectome) was compared against neuronal tracer-based connectivity, as schematically illustrated in Figure 1. Connectome reconstruction performance for the different tractography algorithms was evaluated with three different measures. (I) Sensitivity, which is the proportion of correctly identified present connections (also known as true positive rate; TPR), i.e. the number of true positives (TP) divided by either the number of present connections (P) or the summation of true positives and false negatives (FN):

$$
\text { Sensitivity }(T P R)=T P / P=T P /(T P+F N)
$$

(II) Specificity, which is the proportion of correctly identified absent connections (also known as the true negative rate; TNR), i.e. the number of true negatives (TN) divided by either the number of non-present connections $(\mathrm{N})$ or the summation of true negatives and false positives (FP):

$$
\text { Specificity }(T N R)=T N / N=T N /(T N+F P)
$$

Receiver operating characteristic (ROC) curves were created by plotting the sensitivity (TPR) against the specificity (false positive rate; FPR).

(III) The Jaccard index. Because brain networks have a sparse density, sensitivity and specificity are not equally weighted. False positives are more abundant than false negatives, which is not directly reflected by sensitivity and specificity. The Jaccard index, which is one of the most commonly used similarity coefficients (Yin and Yasuda, 2006), respects the sparse network density of brain networks by not taking into account the number of true negatives, and determines the amount of overlap between the modeled and ground truth network based on the number of available connections in one or both of the networks:

Jaccard index $(J)=T P /(T P+F P+F N)$

\section{Comparison across fiber lengths}

To examine the performance of diffusion-based tractography over different distances we calculated the Euclidean distance for each pair of regions. The Euclidean distance allowed us to determine the distance between all pairs of regions, irrespective of connecting streamlines. First, $x-, y$ - and $z$-coordinates (in $\mathrm{mm}$ ) of the center of gravity of all regions were extracted from the rat brain atlas. Subsequently, the three-dimensional Pythagorean Theorem was applied to calculate the Euclidean distance between all pairs of regions, e.g. for regions $A$ and $B$ :

$$
\text { Euclidean distance }=\sqrt{\left(x_{A}-x_{B}\right)^{2}+\left(y_{A}-y_{B}\right)^{2}+\left(z_{A}-z_{B}\right)^{2}}
$$


All region pairs were subdivided into distance categories (i.e. $<2 \mathrm{~mm}, 2-3 \mathrm{~mm}, 3-4 \mathrm{~mm}, 4-5$ mm, 5-6 mm, 6-7 mm, 7-8 mm, 8-9 mm, 9-10 mm, 10-11 mm, 11-12 mm, 12-13 mm, 13-14 $\mathrm{mm},>14 \mathrm{~mm}$ ). We determined sensitivity, specificity and the Jaccard index of DT-based, CSD-based and global tractography for each distance category (i.e. as a proxy of fiber length).
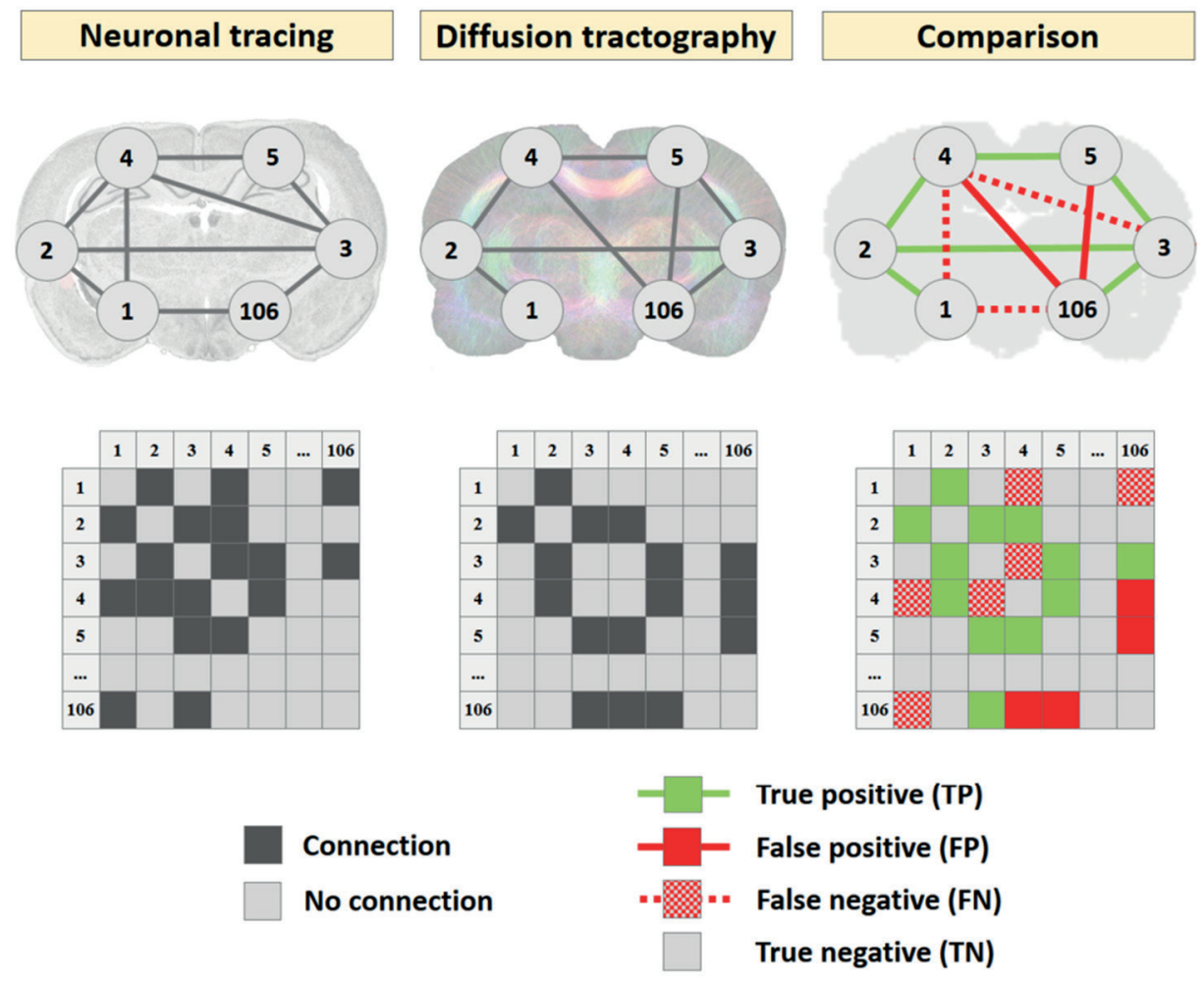

Figure 1. Comparison of connectivity networks from neuronal tracer database and diffusion tractography algorithms. Neuronal tracer-based (left column) and diffusion tractography-based (middle column) connectivity networks represented as network graphs (top), in which nodes represent cortical atlas regions $(\mathrm{N}=106)$ and edges represent connections, and as adjacency matrix (bottom), in which rows and columns represent cortical regions and dark squares represent connections. Diffusion tractography-based connectivity networks were compared against the neuronal tracer-based network as ground truth, which yielded true positives (green lines and squares), false positives (red lines and squares), false negatives (dotted red lines and squares), and true negatives (no line and color-coding) (right column).

\section{Group-level thresholding of connectivity matrix}

To assess the effect of group average-based connectome reconstruction, which may improve signal-to-noise by including consistently found connections and controlling for 
particularities of individual rat networks, we included connections based on incidence over all rats $(\mathrm{N}=10)$ using different thresholds. For example, thresholding at 0.5 means that for a connection to be included in the final connectome reconstruction at least 5 out of the 10 rats should have a particular connection. We varied thresholds between 0.1 (i.e. a connection has to be found in only one rat to be included) and 1 (i.e. a connection has to be found in all rats to be included), and examined the incidence threshold effect on sensitivity, specificity and the Jaccard index.

\section{Streamline thresholding of connectivity matrix}

To assess the effect of streamline-thresholding, we thresholded connections at different streamline numbers, i.e. 2, 5, 10, 15, 20 and 25 streamlines. Regions with a sub-threshold number of linking streamlines were regarded as not connected (i.e. put at zero). We examined the effects of streamlines-thresholding on sensitivity, specificity and the Jaccard index.

\section{Statistical analyses}

All descriptive statistical analyses and visualizations were performed in $R 3.2$ (http://www.rproject.org/), using the packages ggplot2, plyr, caret, igraph, network, reshape2 and sna.

\section{RESULTS}

\section{Tractography maps}

Figure 2 shows representative examples of fiber reconstructions (streamlines) obtained from the three different tractography algorithms applied to high-resolution rat brain diffusion MRI data. The tractography maps displayed various neuroanatomical pathways with high detail. However, subtle differences in fiber patterns between the three algorithms were apparent. DT-based and global tractography-based streamlines show mostly smoothly delineated fibers, whereas CSD-based streamlines show a more irregular pattern with many crossing fibers. Also the appearance of reconstructed fibers in different layers of the hippocampal area differ between the three algorithms. 


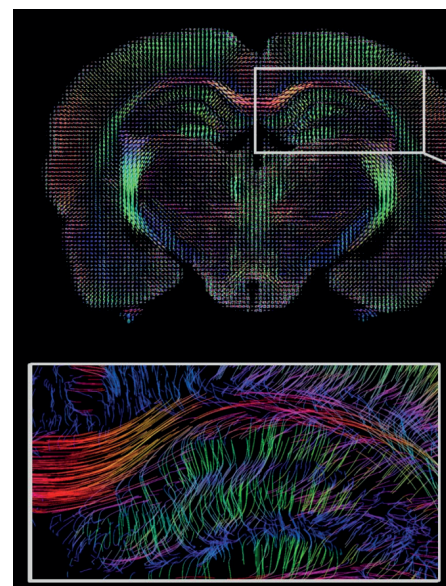

DT-based

streamlines
Fiber orientation

distribution
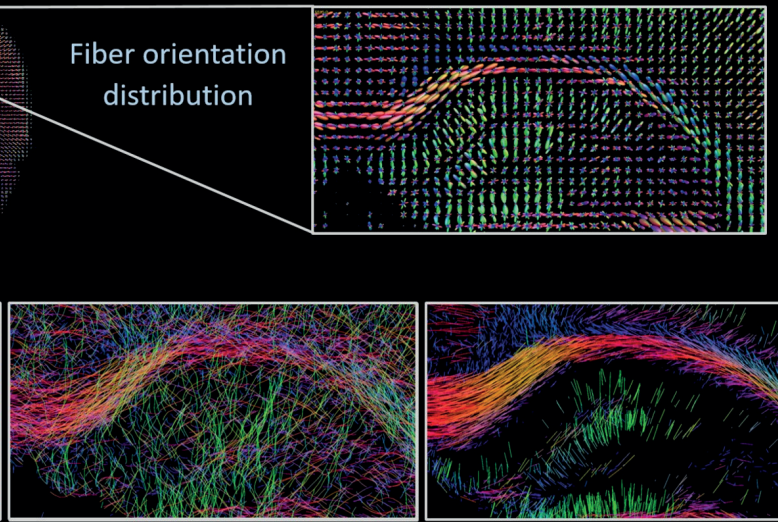

CSD-based

streamlines

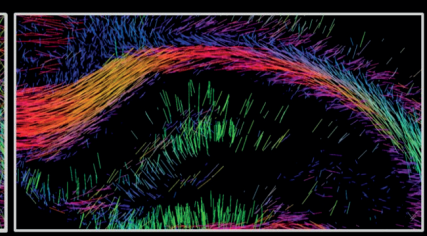

GT-based

streamlines

Figure 2. Tractography from high-resolution diffusion MRI of postmortem rat brain. Top: Coronal rat brain slice displaying fiber orientation distributions, with an enlarged view of the dorsal hippocampal area. Bottom: Representative examples of tract reconstructions in the dorsal hippocampal area, computed with constrained spherical deconvolution-based (DT; left), diffusion tensor-based (CSD; middle) and global tractography algorithms (GT; right).

\section{Accuracy of diffusion tractography-based reconstructions}

To assess the performance of the different diffusion-based tractography algorithms we validated whole brain connectome reconstructions with neuronal tracer data at the connection level, i.e. by comparing the presence or absence of connections between all possible pairs of regions. This yielded the number of true and false positives as well as true and false negatives, and the resulting sensitivity, specificity and Jaccard index at connectome level. Figure 3 (top) shows reconstruction sensitivity against specificity for the three tractography algorithms. For DT-based tractography, sensitivity against specificity is shown as a function of FA threshold at the default step size (i.e. $15 \mu \mathrm{m}$ ), and for CSD-based tractography as a function of FOD threshold at the default step size (i.e. $75 \mu \mathrm{m}$ ). For global tractography, sensitivity against specificity is shown as a function of connection potential. Figure 3 (bottom) shows the Jaccard index as a function of FA threshold (for DT-based tractography), FOD threshold (for CSD-based tractography) and connection potential (for global tractography). CSD-based tractography led to the highest sensitivity for both interand intrahemispheric connections, but the lowest specificity, whereas global tractography resulted in the lowest sensitivity for both inter- and intrahemispheric connections, but the highest specificity. In general, the Jaccard index was higher for both CSD-based and global tractography (specifically at higher connection potentials) but lower for DT-based tractography. 


\section{DT-based tractography}

Figure 3 (left) shows reconstruction sensitivity against specificity of DT-based tractography as a function of FA threshold for different angle thresholds, as a representative example. At connectome level and over all sets of parameter settings, sensitivity varied from $0.22 \pm 0.04$ to $0.61 \pm 0.02$, and specificity from $0.60 \pm 0.02$ to $0.88 \pm 0.02$ for intrahemispheric connections (mean \pm SD (at group level)). For interhemispheric connections sensitivity ranged from $0.09 \pm 0.02$ to $0.42 \pm 0.04$, and specificity from $0.75 \pm 0.02$ to $0.96 \pm 0.01$. Over this range the network density varied from 0.07 to 0.39 . Increase in network density was associated with increases in sensitivity (more true positives), as well as to decreases in specificity, due to more false positives (data not shown).

The FA threshold affected tract reconstruction sensitivity as well as specificity. Increasing FA thresholds (i.e. more strict fiber tracking), e.g. at an angle threshold of $40^{\circ}$ and default step size $(15 \mu \mathrm{m})$, decreased sensitivity from 0.58 to 0.35 for intrahemispheric and from 0.36 to 0.16 for interhemispheric connections, whereas specificity increased from 0.63 to 0.80 for intrahemispheric and from 0.80 to 0.90 for interhemispheric connections. Similarity (i.e. overlap) decreased as indicated by the Jaccard index from 0.32 to 0.24 and from 0.16 to 0.10 for respectively intra- and interhemispheric connections. Over all sets of parameter settings, an increase in number of streamlines led to higher sensitivity and lower specificity values. For example, at an FA threshold of 0.15 , step-size of $15 \mu \mathrm{m}$ and angle threshold of $50^{\circ}$, from 25,000 to 250,000 streamlines, intrahemispheric sensitivity increased from 0.29 to 0.44 whereas specificity decreased from 0.85 to 0.72 . At the same time, the Jaccard index increased with increasing number of streamlines from 0.22 to 0.28 . Similar patterns were seen for interhemispheric connections (data not shown). This increase of the Jaccard index is again explained by a considerable increase in true positives (TP) and a decrease in false negatives (FN) (data not shown). The angle threshold and step size had minimal influence on reconstruction sensitivity and specificity. There was a pattern of increasing sensitivity and decreasing specificity at increasing angle thresholds for the lowest FA threshold. 


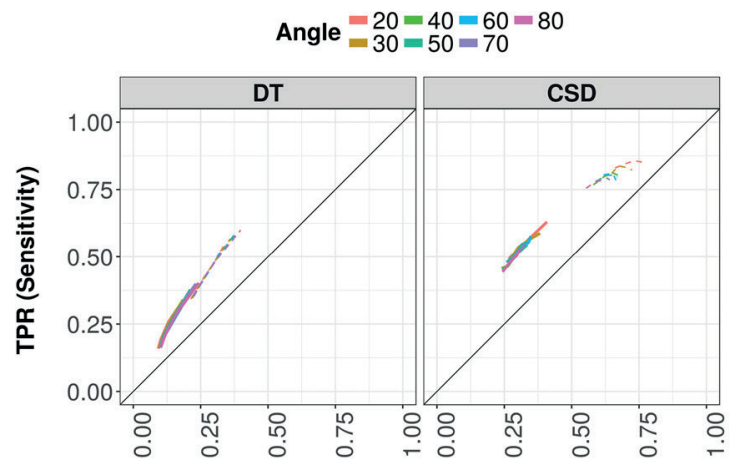

FPR (1-Specificity)

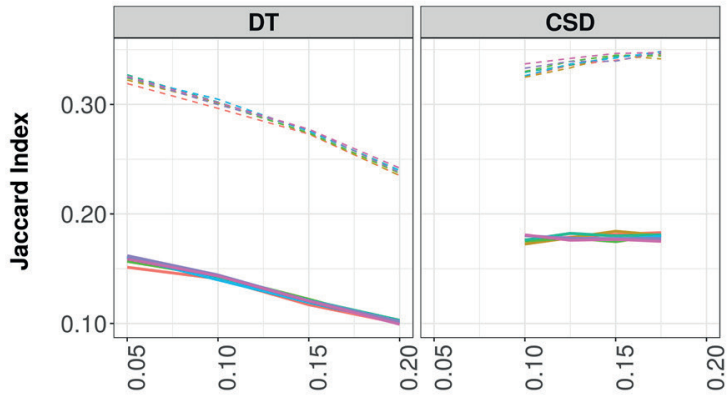

FA threshold

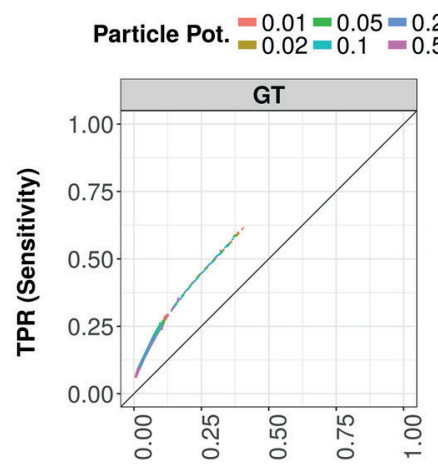

FPR (1-Specificity)

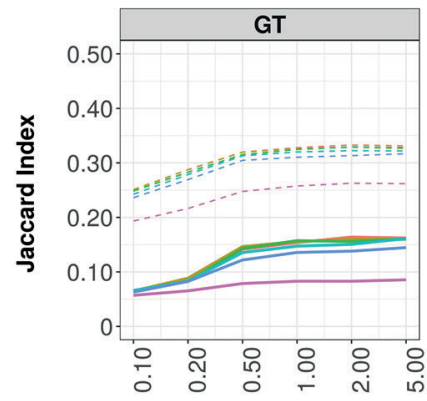

Connection Potential

Figure 3. Connectome reconstruction sensitivity, specificity and Jaccard index of DT-based (left), CSD-based (middle) and global tractography (GT) (right). Left and middle graphs: Reconstruction sensitivity (true positive rate; TPR) versus 1-specificity (false positive rate; FPR) (top) and Jaccard index (bottom) over FA thresholds (DT-based tractography) and over FOD thresholds (CSD-based tractography), for different angle thresholds (line color) with default step size and 250,000 streamlines. Right graphs: GT-based reconstruction sensitivity versus 1-specificity (top) and Jaccard index (bottom) over connection potentials for different particle potentials (line color). All parameters are plotted for interhemispheric (solid lines) and intrahemispheric (dashed lines) connections separately.

\section{CSD-based tractography}

Figure 3 (middle) shows reconstruction sensitivity against specificity of CSD-based tractography as a function of FOD threshold for different angle thresholds, as a representative example. At connectome level and over all sets of parameter settings, sensitivity varied from $0.44 \pm 0.04$ to $0.86 \pm 0.02$, and specificity from $0.23 \pm 0.02$ to $0.78 \pm 0.02$ for intrahemispheric connections (mean $\pm S D$ (at group level)). For interhemispheric connections sensitivity ranged from $0.15 \pm 0.02$ to $0.63 \pm 0.05$, and specificity from $0.60 \pm 0.05$ to $0.95 \pm 0.01$. The network density ranged from 0.13 to 0.68 over all network reconstructions. 
The FOD threshold had minimal effect on tract reconstruction sensitivity and specificity. However, sensitivity and specificity varied to some extent over different angle thresholds, with a generally declining pattern for sensitivity and an increasing pattern for specificity at increasing angle thresholds. With increasing angle thresholds (i.e. from 20 to $80^{\circ}$ ), at an FOD threshold of 0.1 , interhemispheric sensitivity decreased from 0.63 to 0.53 while specificity increased from 0.59 to 0.69 . Intrahemispheric sensitivity decreased from 0.85 to 0.78 whereas specificity increased from 0.24 to 0.37 . Over the same range, the Jaccard index slightly decreased from 0.18 to 0.17 for interhemispheric connections while it slightly increased from 0.32 to 0.34 for intrahemispheric connections. However, there were more false positives and true positives at an angle of $80^{\circ}$ for both intra- and interhemispheric connections (data not shown). Over all sets of parameter settings, we measured slightly higher sensitivity, lower specificity and higher Jaccard index values with increasing number of streamlines, explained by an increase in true positives and a decrease in false negatives.

\section{Global tractography}

Figure 3 (right) shows reconstruction sensitivity against specificity of global tractography as a function of connection potential for different particle potentials. At connectome level and over all sets of parameter settings, sensitivity varied from $0.24 \pm 0.02$ to $0.62 \pm 0.05$, and specificity from $0.59 \pm 0.03$ to $0.90 \pm 0.01$ for intrahemispheric connections (mean \pm SD (at group (evel)). For interhemispheric connections, sensitivity ranged from $0.06 \pm 0.01$ to $0.29 \pm 0.03$, and specificity from $0.87 \pm 0.02$ to $0.99 \pm 0.01$. The density of global-tractography based networks ranged from 0.07 to 0.34 .

Sensitivity and specificity were mostly affected by the connection potential. At a particle potential of 0.01 sensitivity increased from 0.07 to 0.29 (interhemispheric) and from 0.33 to 0.62 (intrahemispheric), while specificity decreased from 0.99 to 0.87 (interhemispheric) and from 0.84 to 0.59 (intrahemispheric) with increasing connection potentials (i.e. more connections being formed). The Jaccard index increased from 0.07 to 0.16 for interhemispheric connections and from 0.25 to 0.33 for intrahemispheric connections. Again, the number of true positives increased together with the number of false positives, although there were generally more false positives (data not shown). Particle potential (i.e. amount and distribution of particles) showed less influence on sensitivity and specificity, although for higher particle potentials (i.e. 0.2 and 0.5 ) sensitivity was lower and specificity tended to be higher.

\section{Effect of SIFT on connectome reconstruction}

Figure 4 shows sensitivity against specificity, and the Jaccard index over FA thresholds (DTbased tractography) and FOD thresholds (CSD-based tractography) for four different angle thresholds. For both DT- and CSD-based tractography, sensitivity decreased whereas 
specificity increased after SIFT. The Jaccard index decreased after applying SIFT. For CSDbased tractography, at an angle threshold of $40^{\circ}$, the interhemispheric sensitivity ranged from 0.42 to 0.54 and the specificity from 0.68 to 0.78 , whereas for CSD-SIFT-based tractograms, sensitivity ranged from 0.26 to 0.34 and specificity from 0.83 to 0.90 . Similar patterns were found for intrahemispheric connections as well as for DT-based and DT-SIFTbased tractograms.
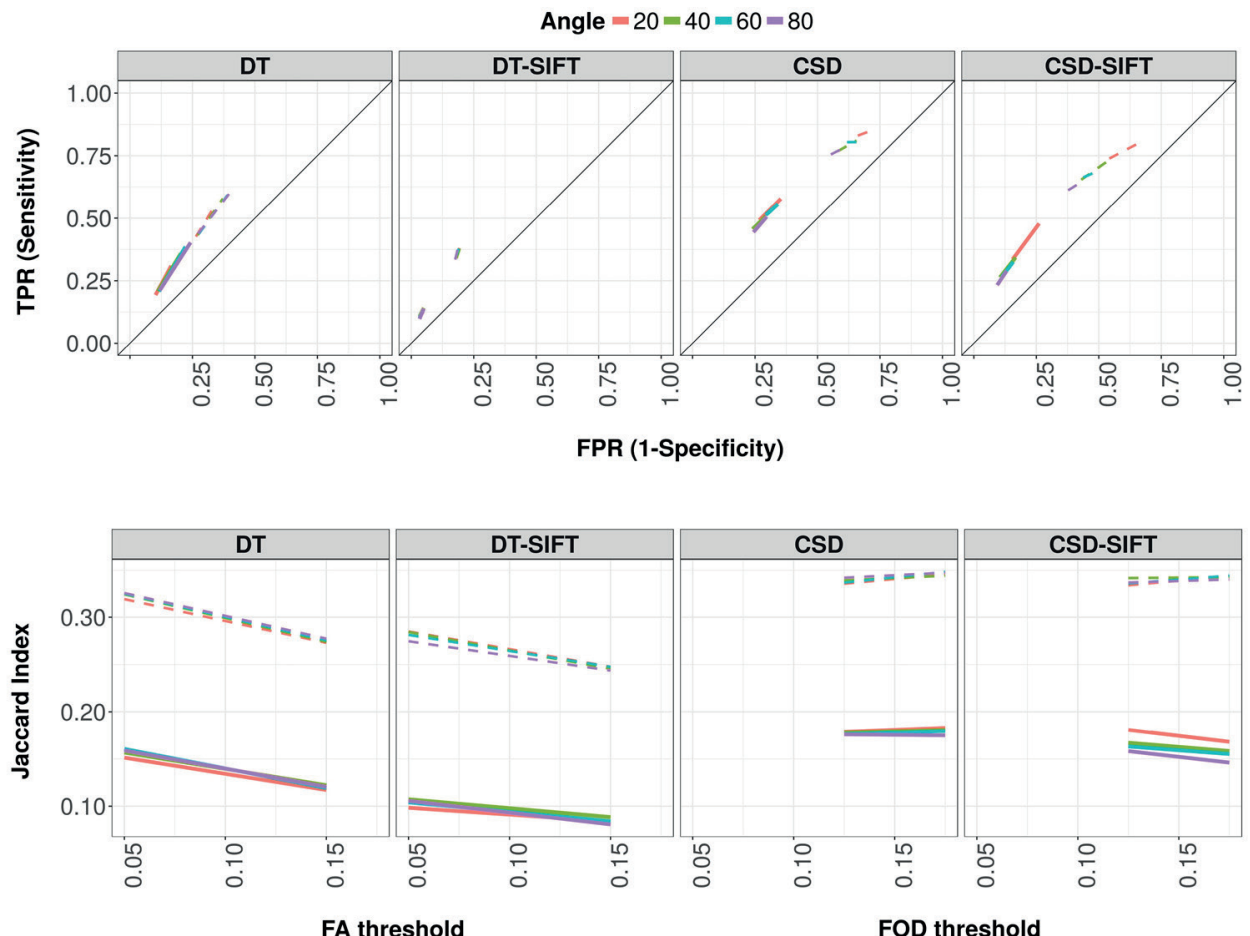

Figure 4. Connectome reconstruction sensitivity, specificity and Jaccard index of DT-based and CSDbased tractography, with and without SIFT correction. Reconstruction sensitivity (true positive rate; TPR) versus 1-specificity (false positive rate; FPR) (top) and Jaccard index (bottom) over FA thresholds (DT and DT-SIFT) and over FOD thresholds (CSD and CSD-SIFT) for different angle thresholds (line color) with default step size and 250,000 streamlines. All parameters are plotted for interhemispheric (solid lines) and intrahemispheric (dashed lines) connections separately.

\section{Inter- vs. intrahemispheric connections}

We determined reconstruction sensitivity and specificity separately for intra- and interhemispheric connections. For all three algorithms, and over all parameter settings, the sensitivity of detecting intrahemispheric connection detection was consistently higher than the sensitivity of interhemispheric connection detection. In contrast, specificity was lower for detection of intrahemispheric connections than for interhemispheric connections. The 
Jaccard index for intrahemispheric connections was consistently higher as compared to its value for interhemispheric connections.
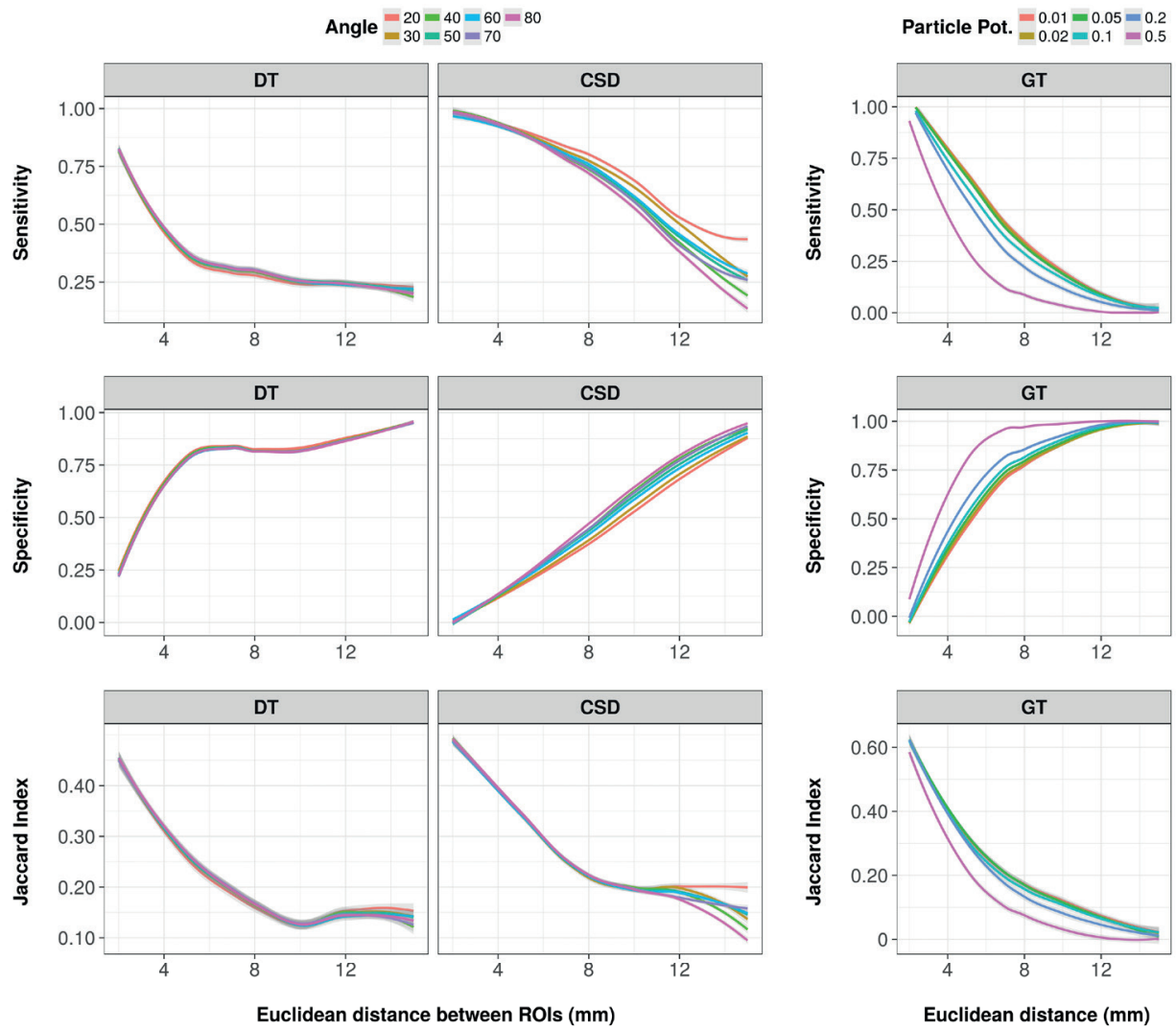

Figure 5. Reconstruction sensitivity, specificity and Jaccard index of DT-based (left), CSD-based (middle) and global tractography (GT) (right). Sensitivity (top), specificity (middle) and Jaccard index (bottom) over Euclidean distance $(\mathrm{mm}$ ) for DT-based (step size $=15 \mu \mathrm{m}$, FA threshold $=0.15$ ) and CSDbased tractography (step size $=75 \mu \mathrm{m}$, FOD threshold $=0.125$ ) with 250,000 streamlines and different angle thresholds (line color), and for GT (connection potential $=1$ ) with different particle potentials (line color).

\section{Effect of fiber length}

We divided all pairs of connected regions in distance categories for which we determined the tract reconstruction sensitivity, specificity and Jaccard index. Figure $\mathbf{5}$ shows the average sensitivity, specificity and Jaccard index for DT-based and CSD-based tractography (over all angles at 250,000 streamlines and default step sizes) as well as for global tractography (over all particle potentials and at a connection potential of 1 ). Overall, with increasing fiber length there was a non-linear decrease in sensitivity, be it with different patterns for DT- 
based, CSD-based and global tractography, together with a non-linear increase in specificity. Also, the Jaccard index showed a non-linear decrease with increasing inter-regional distance. Noteworthy was the relatively low sensitivity $(<0.10)$ and Jaccard index $(<0.05)$ of global tractography for tracking long-distance fibers. Long-distance streamlines were even lacking in some sets of parameter settings (e.g. at a connection potential of 0.1 ) for global tractography.

\section{Effects of group-based analysis}

To analyze the effects of group incidence thresholds, we thresholded an average network at connection level (i.e. based on the number of rats having that specific connection) for DT-based, CSD-based and global tractography. For all tractography algorithms we found that with increasing threshold (i.e. including connections with increasing incidence - or consistency - across rats) connectome reconstruction sensitivity decreased. At the same time, specificity increased. Figure 6 (left panel) shows sensitivity against specificity (top), and Jaccard indices (bottom) for DT-based tractography (using 250,000 streamlines and an FA threshold of 0.2 ). Intrahemispheric sensitivity decreased from 0.75 to 0.20 while specificity increased from 0.44 to 0.92 and interhemispheric sensitivity decreased from 0.52 to 0.04 , whereas specificity increased from 0.60 to 0.99 . Similar patterns were found for CSD-based and global tractography (Figures S2-S6). For DT-based tractography the Jaccard index decreased for both intrahemispheric (from 0.35 to 0.17 ) and interhemispheric connections (from 0.15 to 0.04 ). Similarly, the Jaccard index of interhemispheric and intrahemispheric connections based on CSD-based tractography showed decreasing patterns, although there were peaks depending on streamline number and location (e.g. interhemispheric connections at 250,000 streamlines and an FOD threshold of 0.175 led to the highest Jaccard index at an incidence threshold of 0.4) (Figure S5). Also the Jaccard index of inter- and intrahemispheric connections decreased for global tractography, although different patterns can be seen for different particle potentials (i.e. higher particle potentials led to lower Jaccard indices) (Figure S6).

\section{Effects of streamline thresholds}

To analyze the effects of streamline thresholds, we thresholded networks at connection level (i.e. based on the number of streamlines between two regions) for a specific set of parameter settings for DT-based, CSD-based and global tractography (as representative examples), of which connectivity matrices are shown in Figures S7-S9. These connectivity matrices reveal differences in computed connectivity between tractography methods at the level of individual connections. Sensitivity against specificity over different streamline thresholds (i.e. 1, 2, 5, 10, 15, 20 and 25) are shown in Figure 6 (top right) for specific sets of parameter settings (as representative examples) for the three different algorithms. 


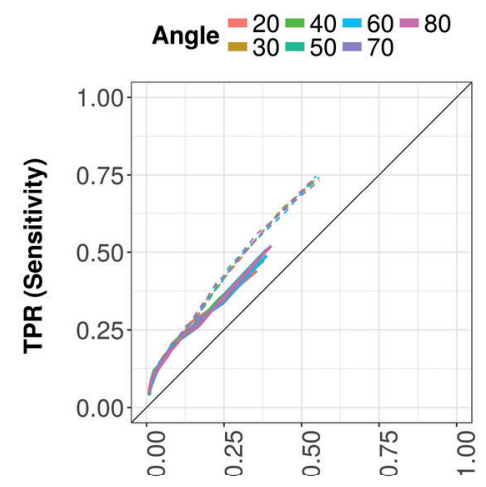

FPR (1-Specificity)

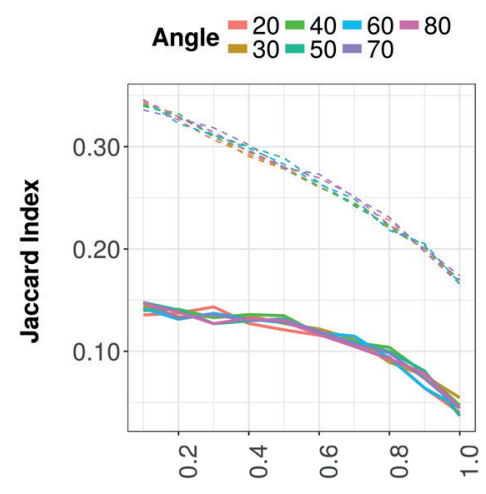

Group incidence threshold
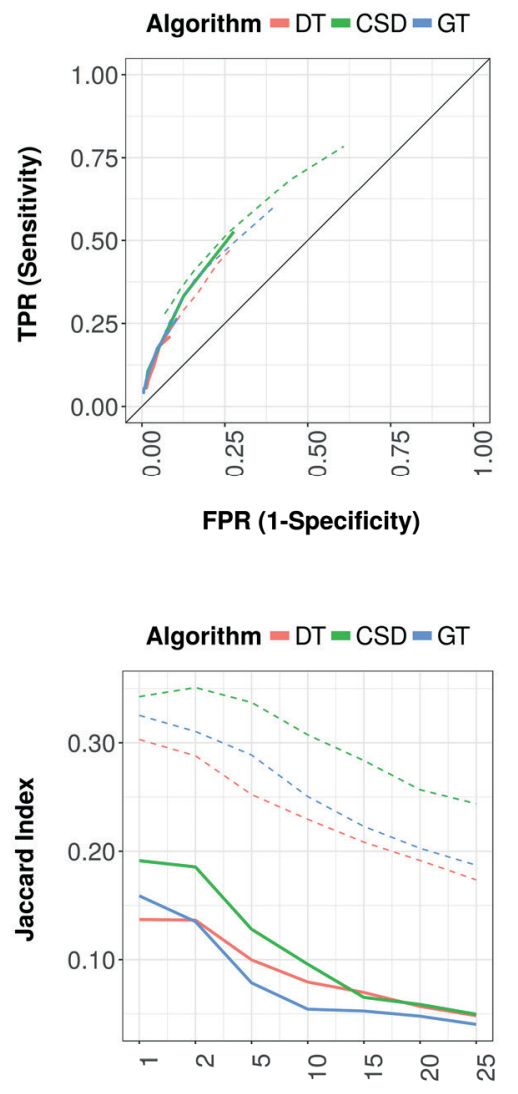

Streamline threshold

Figure 6. Reconstruction sensitivity, specificity and Jaccard index of DT-based tractography at different group-based incidence thresholds and streamline thresholds. Left graph: Reconstruction sensitivity (true positive rate; TPR) versus 1-specificity (false positive rate; FPR) (top) and Jaccard index (bottom) over group incidence thresholds with different angle thresholds (line color) for DT-based tractography (step size $=0.15 \mu \mathrm{m}$, FA threshold $=0.15$ and 250,000 streamlines) (left graphs). Right graph: Reconstruction sensitivity (true positive rate; TPR) versus 1-specificity (false positive rate; FPR) (top) and Jaccard index (bottom) over streamline thresholds for DT-based tractography (red; step size $=15 \mu \mathrm{m}$, FOD threshold $=0.15$ ), CSD (green; step size $=75 \mu \mathrm{m}$, FA threshold $=0.125$ ), with an angle threshold of $40^{\circ}$ and 250,000 streamlines, and for global tractography (GT) (blue; connection potential $=1$, particle potential $=0.01$ ). All parameters are plotted for interhemispheric (solid lines) and intrahemispheric (dashed lines) connections separately. 
For all tractography algorithms we found that with increasing streamline threshold, connectome reconstruction sensitivity decreased, whereas specificity increased (Figures S10 and S11). Although the amount of false positive connections decreased with increasing streamline threshold, the number of true positives decreased as well, specifically at higher thresholds (Figures S12 and S13). For DT-based tractography the Jaccard index decreased with increasing streamline thresholds, for both interhemispheric (i.e. from 0.14 to 0.05 ) and intrahemispheric (i.e. from 0.31 to 0.17 ) connections (Figure 6). Similar patterns were found for CSD-based and global tractography (Figure 6).

\section{DISCUSSION}

In this study, we evaluated the accuracy of connectome reconstruction with three different diffusion-based tractography approaches in rat brain. The connectome reconstructions were compared against neuroanatomical tracer data. Despite our ability to track a large variety of brain-wide neuronal fiber connections, we detected a considerable number of false positive and false negative connectivity reconstructions, which increased with increasing fiber lengths. Despite their ability to solve reconstruction issues with crossing fibers, overall performance (i.e. as expressed by the Jaccard index) of CSD-based and global tractography was comparable to conventional DT-based tractography. CSD-based tractography outperformed global tractography for reconstruction of long distance connections. However, the different tractography approaches presented considerable differences in connectome reconstruction sensitivity and specificity. While DT and GT led to low sensitivity and high specificity, CSD resulted in high sensitivity together with low specificity.

The considerable amount of false positive and false negative connections generated by diffusion-based tractography in rat brain is in line with related studies in macaque brain (Azadbakht et al., 2015; Donahue et al., 2016; Thomas et al., 2014), and mouse brain (Calabrese et al., 2015; Chen et al., 2015), and similar findings have been reported for the human connectome (Maier-Hein et al., 2016). We identified several factors that influence the sensitivity and specificity of connectome reconstruction. The number of streamlines (for CSD- and DT-based tractography) and connection potential (GT) were positively related to sensitivity. Both factors increased the total number of reconstructed connections, leading to a higher true positive rate. Conversely, FA threshold (for DT-based tractography), particle potential (GT), group-based average network thresholding, streamline thresholding and filtering (SIFT) were negatively related to sensitivity. The step size and angle threshold (for CSD- and DT-based tractography) and FOD threshold (for CSD-based tractography) had almost no effect. Improvements in sensitivity were invariably at the expense of specificity and vice versa. 
The FA threshold had significantly more effect on connectome reconstruction sensitivity and specificity than the step size and angle threshold, in line with previous research (Azadbakht et al., 2015; Chen et al., 2015). In fact, an increasing FA threshold negatively influenced sensitivity and the Jaccard index, suggesting that an FA threshold should be omitted for this type of datasets and analyses (Azadbakht et al., 2015; Chen et al., 2015). Apparently, the angle thresholds and step sizes - both important factors related to tract curvature - as applied in our study were within plausible neuroanatomical ranges, thereby not affecting the overall connectome reconstructions. However, the angle threshold might still have affected specific pathway reconstructions in the brain (Thomas et al., 2014). Also, further increase (or decrease) of the step size and angle threshold may affect, on their own, or by interaction, subsequent connectome reconstruction.

Interestingly, contrary to the FA threshold (for DT-based tractography), the FOD threshold (for CSD-based tractography) appeared to have no impact on connectome reconstruction sensitivity and specificity. This may be explained by the different nature of DT-based tractography (i.e. deterministic) and CSD-based tractography (i.e. probabilistic) or by the different nature of the FA and FOD thresholds. The FA threshold terminates streamlines in voxels with sub-threshold FA values (i.e. at voxel level), whereas the FOD threshold only prohibits streamline sampling from sub-threshold FOD amplitude peaks within a voxel (i.e. at sub-voxel level), while sampling from other (criteria fulfilling) FOD peaks is still possible. Nevertheless, we expected that an increase in FOD threshold would lead to more rigid delineation of tracts, since streamlines will only follow directions with higher FOD amplitudes (Tournier et al., 2012). However, inspection of our data revealed that many FODs, representing (multiple) crossing fibers, contained multiple high FOD amplitudes. Therefore, to influence the connectome reconstruction sensitivity and specificity we should apparently increase the FOD thresholds to much higher values (i.e. far beyond the default 0.1 threshold), but this would probably lead to undesirable exclusion of many (truly) crossing fibers. Also, it should be noted that the effects of FOD threshold adjustments may be different across species and datasets.

SIFT filtering has been developed to improve the biological plausibility of final structural connectome reconstructions (Smith et al., 2013, 2015a). However, our study did not reveal an improvement in correspondence between reconstructed connectomes and neuroanatomical connectivity after SIFT correction. As such, our overall results confirm previously reported trade-offs between sensitivity and specificity of tract reconstructions across tractography methods (Bastiani et al., 2012; Chen et al., 2015; Donahue et al., 2016; Thomas et al., 2014). However, we found, as indicated by differing Jaccard indices that the trade-off between reconstruction sensitivity and specificity varies considerably over a range of settings and streamline- or group-based thresholds. This means that a change in the 
number of false positives through adjustment of tractography parameter settings is not linearly scaled with the change in number of false negatives (and true positives).

Our analyses revealed clear differences in reconstruction sensitivity and specificity dependent on the site (running within or between hemispheres) and length of connections. This is similar to what has previously been reported for macaque brain (Thomas et al., 2014), where reconstruction sensitivity and specificity differed between distinct pathways (and sites) in the brain. The Jaccard index generally increased with higher number of streamlines for intra- and interhemispheric tract reconstructions, despite an increase in false positive tract reconstructions. Our tested DT-based (i.e. deterministic) and CSD-based (i.e. probabilistic) tractography algorithms were affected by connection distance, showing a non-linear decrease in sensitivity and Jaccard index, and a non-linear increase in specificity, with increasing connection distance. CSD-based and global tractography yielded highest Jaccard indices for short-distance connections, which decreased for connections of longer distance. In agreement with previous studies (Jbabdi et al., 2015; Zalesky and Fornito, 2009), we found a relatively high rate of false negative reconstructions for connected cortical areas located at large distance from each other, especially for global tractography, which may consequently lead to omissions in identification of important inter-modular hub-node connections. Future studies should focus on elucidation of the exact underlying causes of these variations between and within deterministic and probabilistic tractography approaches. Despite the general trade-off between reconstruction sensitivity and specificity, we observed notable differences between tractography algorithms and parameter settings that should be taken into account for application in connectivity studies and subsequent network analyses. For example, if avoidance of false positives and false negatives are equally important, it may be advisable to choose tractography parameter settings that yield the highest Jaccard index (i.e. a high number of streamlines and a low FA threshold). On the other hand, for modular connectomes such as brain networks, certain network metrics, e.g. clustering coefficient, global efficiency and modularity, have been shown to be more strongly affected by false positives (which tend to arise inter-modularly) than by false negatives (which tend to arise intra-modularly) (Drakesmith et al., 2015; Zalesky et al., 2016). Under such circumstances an approach that overweighs reconstruction specificity over sensitivity (e.g. with global tractography using a low connection potential, or by applying streamline thresholds to increase specificity), thereby minimizing false positives at the expense of uncovering some true connections, may be preferred (Fornito et al., 2013; van Wijk et al., 2010; Zalesky et al., 2016). Zalesky et al. have recommended that graph-based network analyses should be preferably performed on connectomes with at least about twice the number of false negatives compared to false positives (i.e. the 2:1 rule) (Zalesky et al., 2016). Our data show that specific DT and global tractography parameter settings can satisfy this rule. However, this may be more complicated for CSD, 
which typically generates high density networks and relatively large numbers of false positives. Lastly, reconstruction sensitivity and specificity may be influenced by streamline thresholding or group-based analysis (i.e. incidence thresholds, which might increase robustness against inter-subject variation), which might be employed to decrease the number of false positive reconstructions to satisfy the suggested 2:1 rule (Zalesky et al., 2016).

It is possible that the mismatch between diffusion-based tractography and neuroanatomical tracers in our (and previous) work may be partly due to incompleteness of neuroanatomical tracer data. For example, the multi-synaptic connections, which are not easily detected with neuronal tracing, might be incorrectly identified as false positives. This actually challenges the concept of neuronal tracing as gold standard method for assessment of neuroanatomical connectivity. It is also conceivable that diffusion-tractography findings may guide future neuronal tracing assessments of unknown neuronal pathways. We also did not assess connectivity of subcortical regions, which are less easily co-registerable to the rat brain anatomical atlas, which biases our conclusions towards cortical connectomics. Another point is that we only considered streamline endpoints in determining connectivity between two regions of interest, thereby ignoring the three-dimensional structure of anatomical pathways. It may be argued that this is inappropriate from an anatomical viewpoint, however, this has been the strategy in the majority of structural network studies (which typically focus on network topology and not on exact fiber trajectories). Moreover, a recent study in mice showed that the accuracy of diffusion-based tractography is worse when spatially comparing tractography streamlines with tracer-based connections using 3D co-localization analysis (Calabrese et al., 2015). Lastly, the weights of neuronal connections were not considered in our study. While this was recently done to compare measures of connectivity strength between diffusion tractography and tract tracing data from macaque brain (Donahue et al., 2016; van den Heuvel et al., 2015), most neuroanatomical connections are at least to a certain degree bidirectional and contain nested sub-regions with their own connections and corresponding weights. This complicates the assignment of an absolute or relative measure of connection weight and directionality.

In conclusion, our study shows that conventional deterministic DT-based and more advanced probabilistic CSD-based and global tractography approaches reconstruct the whole-brain connectome with comparable accuracy, be it with considerable amounts of false positive and false negative connections, which strongly depends on the algorithm and parameter settings. Because tractography conditions differ between intra- and interhemispheric connections as well as over different connection distances, algorithms should be chosen purposefully and, if possible, combined to enable most reliable reconstruction of connectomes at large-scale network or whole-brain level. For instance, since global tractography has difficulties with resolving long-distance connections, this long- 
distance connectivity information could be extracted from different adjacency matrices (based on DT-based tractography). The large number of invalid tract reconstructions we observed in our study, in line with a recent study on human tract reconstructions (MaierHein et al., 2016), stresses the relatively ill-posed nature of current tractography approaches and the need for methodological progression. Use of alternative streamline filtering techniques such as SIFT2 (Smith et al., 2015b), inclusion of anatomical constraints (Lemkaddem et al., 2014; Smith et al., 2012) or priors (Christiaens et al., 2014, 2015b; Yendiki et al., 2011), or application of Bayesian connectomics (Hinne et al., 2012; Kasenburg et al., 2016) may lead to further improvement of connectome reconstruction accuracy. Furthermore, the increasing availability of neuronal tracer databases may aid in fine-tuning of diffusion-based tractography settings and applications, which will contribute to the progress of the rising field of connectomics. 


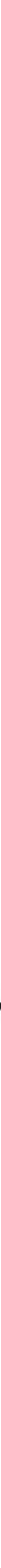




\section{Chapter 4}

\section{Modified Structural Network Backbone in the Contralesional Hemisphere Chronically after Stroke in Rat Brain}

M.R.T. Sinke, W.M. Otte, M.P.A. van Meer, A. van der Toorn \& R.M. Dijkhuizen 


\section{ABSTRACT}

Functional outcome after stroke depends on the local site of ischemic injury and on remote effects within connected networks, frequently extending into the contralesional hemisphere. However the pattern of large-scale contralesional network remodeling remains largely unresolved. In this study we applied diffusion-based tractography and graph-based network analysis to measure structural connectivity in the contralesional hemisphere chronically after experimental stroke in rats. We used the minimum spanning tree method, which accounts for variations in network density, for unbiased characterization of network backbones that form the strongest connections in a network. Ultrahigh-resolution diffusion MRI scans of eight post-mortem rat brains collected 70 days after right-sided stroke were compared against scans from ten control brains. Structural network backbones of the left (contralesional) hemisphere, derived from 42 atlas-based anatomical regions, were found to be relatively stable across stroke and control animals. However, several sensorimotor regions showed increased connection strength after stroke. Sensorimotor function correlated with specific contralesional sensorimotor network backbone measures of global integration and efficiency. Our findings point toward poststroke adaptive reorganization of the contralesional sensorimotor network with recruitment of distinct sensorimotor regions, possibly through strengthening of connections, which may contribute to functional recovery. 


\section{INTRODUCTION}

Stroke regularly results in loss of motor function and is one of the main causes of disability in adults worldwide. Nevertheless, most patients experience partial recovery of sensorimotor function in the weeks and months following the stroke, which is believed to be associated with reorganization of surviving neural networks (Grefkes and Fink, 2011). This provides opportunities for recovery-promoting therapies that target intrinsic neurorestorative mechanisms.

Post-stroke brain reorganization manifests from micro- (e.g. synaptic plasticity) to macroscale (e.g. cortical remapping). Various studies have revealed local microstructural reorganization around brain lesions after stroke, including dendritic branching, synaptogenesis and neurite outgrowth, which affect networks of neuronal connections at a larger scale (Biernaskie and Corbett, 2001; Jones et al., 1996; Stroemer et al., 1995). Poststroke structural remodeling is not limited to perilesional sites and may also extend to the contralesional hemisphere, as has been demonstrated in animal stroke models (Carmichael and Chesselet, 2002; Dancause, 2005; Liu et al., 2008; Marcel et al., 2007) and human stroke patients (Cai et al., 2016; Crofts et al., 2011; Dacosta-Aguayo et al., 2014; Granziera et al., 2012; Gratton et al., 2013; Johansen-Berg et al., 2010; Schaechter et al., 2010). Contralesional structural alterations may be particularly prominent in regions that are homologous to the ipsilesional stroke-affected regions (Crofts et al., 2011; Granziera et al., 2012; Johansen-Berg et al., 2010), and could thereby contribute to partial retrieval of acute stroke-induced loss of specific function (Cai et al., 2016; Crofts et al., 2011; Dacosta-Aguayo et al., 2014; Johansen-Berg et al., 2010; Schaechter et al., 2010). However, the pattern of remodeling of contralesional neuronal circuitry, particularly at meso- and macroscale, remains largely unresolved.

Post-stroke large-scale neural network alterations can be assessed with neuroimaging techniques, such as magnetic resonance imaging (MRI), which offer powerful means to map structural and functional networks non-invasively in animal models and patients (Dijkhuizen et al., 2012; Grefkes and Fink, 2011). Today, the gold standard neuroimaging technique to characterize whole-brain structural connectivity in vivo is diffusion-based tractography (Basser et al., 2000; Jbabdi and Johansen-Berg, 2011). Diffusion imaging characterizes the degree and directionality of tissue water diffusion, which informs on the underlying tissue architecture - particularly the arrangement of axonal projections - and allows 3dimensional tracking of neuronal fibers (Basser et al., 2000). A growing amount of data has demonstrated that diffusion-based tractography may inform on alterations in structural brain connectivity after stroke, possibly associated with neuroplasticity (Crofts et al., 2011; Johansen-Berg et al., 2010; Schaechter et al., 2010). However, to our knowledge, so far only one diffusion-based tractography study has reported on contralesional remodeling of network connectivity in stroke patients (Crofts et al., 2011). 
Neural network features are frequently quantified with graph analysis based on nodes (e.g. predefined brain regions) and edges (e.g. connecting fiber tracts) (Bassett and Bullmore, 2009; Bullmore and Sporns, 2009; Rubinov and Sporns, 2010; Sporns, 2010). However, graph-based comparison of networks with different edge densities and connectivity distributions remains challenging (Fornito et al., 2013; van Wijk et al., 2010). Since focal brain injury typically results in less dense structural networks with fewer connected node pairs, alternative network analysis strategies that are not influenced by variations in network size and density could be more appropriate for the characterization of reorganizing networks after stroke. A promising approach is assessment of the network backbone with minimum spanning tree (MST) analysis, which involves a sub-graph of the original network comprised of the strongest network connections (Jackson and Read, 2010; Stam et al., 2014; Tewarie et al., 2015a). Backbone analysis enables unbiased comparison of networks irrespective of differences in density and connectivity distributions, and has been successfully applied to capture network changes during brain development and in brain disorders such as epilepsy, multiple sclerosis, Alzheimer's disease, brain tumors and schizophrenia (van Dellen et al., 2014; van Diessen et al., 2016; Engels et al., 2015; Otte et al., 2015; Stam et al., 2014; Tewarie et al., 2015b, 2015a).

In the present study we aimed to identify macroscale alterations in structural network connectivity and organization in the contralesional hemisphere chronically after experimental stroke. To that aim we acquired diffusion MRI data of post-mortem brains from healthy control rats and from rats with sensorimotor deficits after unilateral stroke in the middle cerebral artery territory. Post-mortem imaging allows very long scanning times, enabling acquisition of images with ultrahigh spatial resolution and great structural detail, not feasible under in vivo conditions. We measured white matter volume and integrity in the contralesional hemisphere, and analyzed backbones of diffusion tractography-based structural networks in relation to sensorimotor performance.

\section{METHODS}

\section{Stroke model, sensorimotor function and in vivo MRI}

Ethical approval was given by the Animal Experiments Committee of the University Medical Center Utrecht and the Utrecht University, and experiments were performed in accordance with the guidelines of the European Communities Council Directive.

Eighteen adult male Sprague Dawley rats, weighing 280-320g, were included in the study. Eight rats underwent transient focal cerebral ischemia by 90 -min intraluminal occlusion of the right middle cerebral artery, as previously described (Longa et al., 1989; van Meer et al., 2010b). Ten rats served as age-matched controls. Behavioral data and in vivo functional MRI data from these animals have been reported previously (van Meer et al., 2010b). 
All rats were trained at four and three days before stroke, and behaviorally tested at two days before and at three, seven, 21,49 , and 70 days after stroke. A sensorimotor deficit score was acquired through grading on different subscales of 'motility, spontaneous activity' (e.g. moving limbs without proceeding), 'gait disturbances' (e.g. walking toward contralateral side), 'postural signs' (e.g. forelimb flexion) and 'limb placing' (e.g. normal, weak or no placing) (van der Zijden et al., 2008). The total sensorimotor deficit score had a range of 0 (i.e. no deficit) to 20 (i.e. maximal deficit) points.

Infarction size and location were assessed at 70 days after stroke on a 4.7T Varian MR scanner with $\mathrm{T}_{2}$-weighted MRI (multiple spin echo; repetition time (TR)/echo time (TE) = $3000 / 17.5 \mathrm{~ms}$; echo train length $=8$; field of view $=32 \times 32 \mathrm{~mm}^{2}$; matrix $=128 \times 128$; nineteen coronal slices; slice thickness $=1 \mathrm{~mm}$ ). Quantitative $T_{2}$ maps were computed as previously described (van Meer et al., 2010b). Stroke lesions characterized by $T_{2}$ prolongation and tissue liquefaction were manually outlined on $T_{2}$ maps by two independent neuroscientists, and the overlapping area was assigned as infarcted tissue.

\section{Post mortem anatomical and diffusion MRI}

Stroke and control rats were euthanized at day 70 and transcardially perfused with phosphate-buffered saline and $4 \%$ paraformaldehyde. After one year of storage in paraformaldehyde, post mortem brains were fixated in a syringe filled with proton-free perfluoropolyether (Fomblin ${ }^{\circledR}$, Solvay Solexis) to prevent magnetic susceptibility artifacts around the borders of the brain, which facilitates image post-processing.

High angular resolution diffusion imaging of post mortem rat brain was conducted on a 9.4T Varian MR scanner (eight-shot EPI; TR/TE $=6000 / 32 \mathrm{~ms}$; FOV $=25 \times 25 \mathrm{~mm}^{2}$; acquisition matrix = $128 \times 128$; zero-filled to $256 \times 256$; ninety-one coronal slices with slice thickness of $0.2 \mathrm{~mm}$; isotropic voxel resolution $=0.2 \times 0.2 \times 0.2 \mathrm{~mm}^{3}$; four images without diffusionweighting; diffusion-weighted images in two sets of sixty directions equally spaced on a sphere with $b=2871.50 \mathrm{~s} / \mathrm{mm}^{2}$ in opposite gradient directions $(\Delta / \delta=13 / 6 \mathrm{~ms})$; total acquisition time $=13.25 \mathrm{~h} /$ dataset).

\section{Image processing}

Images were pre-processed with FSL 5.0 (http://ww.fmrib.ox.ac.uk/fsl/) (Jenkinson et al., 2012). The image processing pipeline as described below is pictured in Figure 1. For each dataset whole-brain fractional anisotropy (FA) maps were linearly and nonlinearly matched with a custom-built 3D reconstruction of the Paxinos and Watson rat brain atlas (Paxinos and Watson, 2005), using affine and nonlinear registrations (FLIRT/FNIRT) (Andersson et al., 2007; Jenkinson and Smith, 2001). FA maps were determined from the diffusion tensor. The contralesional hemisphere was partitioned in 42 cortical and subcortical network regions based on standard anatomical atlas boundaries (see Figure 1) and projected in subject 
space. Anatomical region details are given in Table 1. A mask that only covered the contralesional hemisphere was created using the $\mathrm{R}$ package oro.nifti [https://cran.rproject.org/web/packages/oro.nifti/index.html]. This $\mathrm{R}$ package provides functions for the input, output and visualization of medical imaging data that follow standard imaging formats. Tractography was done in subject space within the contralesional hemisphere mask.

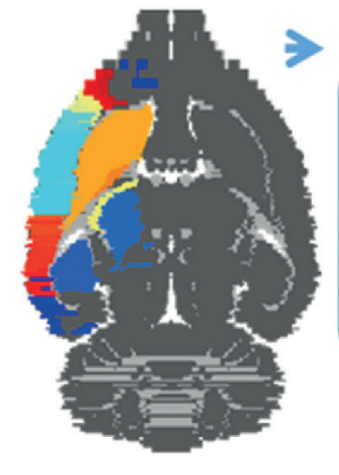

Atlas regions

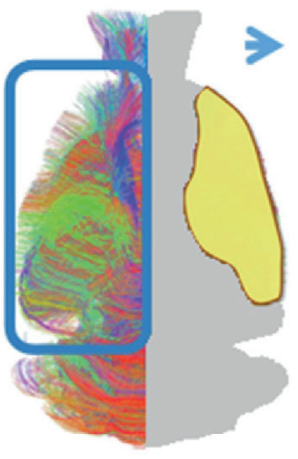

Tractography

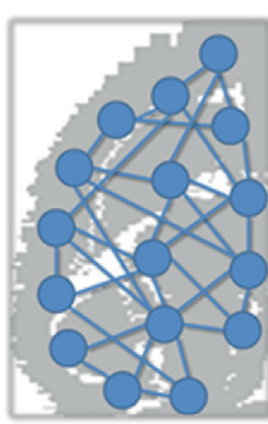

Structural

network

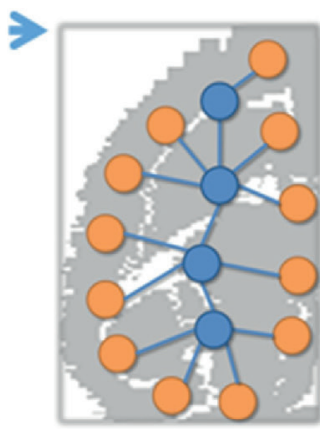

Minimum spanning tree

Figure 1. Image processing pipeline. A schematic overview of the image processing steps. After registration with the Paxinos and Watson rat brain atlas, 42 unilateral brain regions were resampled in subject space and used to extract weighted structural networks from diffusion-based tractography in the contralesional hemisphere. A minimum spanning tree (MST) was extracted from the weighted networks and quantified using MST metrics. Atlas labels are plotted on top of a grayscale image of a transverse rat brain slice (first picture). Color-coded contralesional fiber tracts are shown for a single subject (>5 mm tract length only; approximate lesion site depicted in yellow) (second picture). Magnified illustrations display the contralesional cerebral network with nodes and edges (third picture), and corresponding MST (MST leaf nodes in orange) (fourth picture).

\section{Anatomical characteristics}

A white matter mask was constructed using an FA threshold of 0.2 (i.e. all voxels with a subthreshold value were regarded as grey matter). We determined white and grey matter volumes of the contralesional hemisphere. For white matter voxels we determined the mean FA, radial diffusivity (RD), and axial diffusivity (AD).

\section{Diffusion tensor-based tractography}

Diffusion-based tractography and subsequent connectome reconstructions were performed in MRtrix $3^{\circledR}$ (http://www.mrtrix.org/) (Tournier et al., 2012). We used diffusion tensor-based tractography to reconstruct whole brain white matter fiber connections on a voxel-wise basis from the diffusion MRI data (Basser et al., 1994b, 2000). Seeds were equally 
distributed over the whole contralesional hemisphere, from which tracts were generated using a voxel-by-voxel stepwise approach following the principal diffusion directions. Based on our previous work (Sinke et al., 2018) we selected tractography parameter settings that yield highest specificity for reconstruction of intrahemispheric connections as suggested for network analyses (Zalesky et al., 2016). We used an FA threshold of 0.15 (i.e. tract generation was terminated in sub-threshold voxels), a step-size of $130 \mu \mathrm{m}$, an angular threshold of $20^{\circ}$ (i.e. tracts were terminated when bending with an angle exceeding the threshold) and 25k streamlines. The relative low FA threshold allowed for optimal tract termination within the cortical gray matter regions.

We used spherical-deconvolution informed filtering of tractograms (SIFT) to improve the accuracy of reconstructed sets of white matter bundles by optimizing and fitting them to the underlying diffusion-weighted images (Smith et al., 2013, 2015a). Thereby, the number of tracts is normalized and corrected for density differences across different regions and datasets.

\section{Structural network reconstruction}

Structural connectivity was determined for each pair of atlas regions. Two regions were considered connected if one or more filtered tracts had their end point in both regions. These were subsequently used to construct a weighted network, represented by the graph $S=(N, W)$ where $N$ is the set of 42 unilateral network regions and $W=w_{i j}$ is the $N \times N$ weight matrix, where $w_{i j}$ is set to the SIFT-corrected number of all tracts connecting the region pairs. Self-connections were excluded. The filtered number of tracts was used as a measure of connectivity strength. The density of undirected networks, with no self-connections, was defined as the ratio of the number of existing edges over the total possible number of edges (that is, $N \times(N-1) / 2)$ ).

Weighted connectivity matrices were formed for $42 \times 42$ atlas regions. To examine intersubject consistency of connections, group-based average structural networks were generated from the prevalence of connections (after binarization of individual network matrices (i.e. all connection weights were set to a value of one)) within the control and stroke group.

\section{Sensorimotor network reconstruction}

We extracted fifteen sensorimotor regions from the acquired structural network matrices, i.e. the primary motor cortex (M1), secondary motor cortex (M2), caudate putamen, thalamus, globus pallidus, sub-regions of the primary somatosensory cortex (S1) (i.e. forelimb region, oral dysgranular zone, upper lip region, shoulder region, hindlimb region, dysgranular zone, trunk region, barrel field, jaw region and S1 not otherwise specified) and 
secondary somatosensory cortex (S2). Sensorimotor connectivity matrices were formed from $16 \times 16$ unilateral sensorimotor regions.

\begin{tabular}{|c|c|}
\hline Atlas description & Paxinos and Watson atlas labels \\
\hline Agranular insular cortex - dorsal part & AID \\
\hline Agranular insular cortex - posterior part & AIP \\
\hline \multirow[t]{2}{*}{ Agranular insular cortex - ventral part } & AIV \\
\hline & $\begin{array}{l}\text { ACo, BL, BLA, BLP, BLV, BM, BMA, BMP, Ce, CeC, } \\
\text { CeL, }\end{array}$ \\
\hline Amygdaloid nuclei & $\begin{array}{l}\text { CeM, CeMAD, CeMAV, CeMPV, IM, La, LaDL, LaVL, } \\
\text { LaVM, Me, MeA, MeAD, MeAV, MePD, MePV, } \\
\text { PLCo, PMCo }\end{array}$ \\
\hline Primary auditory cortex & Au1 \\
\hline Secondary auditory cortex - dorsal area & AuD \\
\hline Secondary auditory cortex - ventral area & AuV \\
\hline Cingulate cortex - area 1 & Cg1 \\
\hline Cingulate cortex - area 2 & $\mathrm{Cg} 2$ \\
\hline Caudate putamen & $\mathrm{CPu}$ \\
\hline Dysgranular insular cortex & $\mathrm{DI}$ \\
\hline Frontal cortex - area 3 & Fr3 \\
\hline Granular insular cortex & $\mathrm{GI}$ \\
\hline Globus pallidus & EGP,IGP \\
\hline Hippocampus & $\begin{array}{l}\text { vhc, dhc, GrDG, CA1, CA2, CA3, Or, MoDG, Py, Rad, } \\
\text { SLu, LMol, FC, PoDG }\end{array}$ \\
\hline Lateral parietal association cortex & LPtA \\
\hline Primary motor cortex & M1 \\
\hline Secondary motor cortex & M2 \\
\hline Medial parietal association cortex & MPtA \\
\hline Nucleus accumbens & AcbSh, AcbC, LAcbSh \\
\hline Piriform cortex & Pir \\
\hline Prelimbic cortex & $\operatorname{PrL}$ \\
\hline Parietal cortex - posterior area - dorsal part & PtPD \\
\hline Parietal cortex - posterior area - rostral part & PtPR \\
\hline Retrosplenial cortex & RSGa, RSGb, RSGc, RSD \\
\hline Rhinal cortex & DIEnt, DLEnt, Ect, Ent, LEnt, MEnt, PRh, VIEnt \\
\hline Reticular thalamic nucleus & Rt \\
\hline Primary somatosensory cortex & S1 \\
\hline Primary somatosensory cortex - barrel field & S1BF \\
\hline Primary somatosensory cortex - dysgranular zone & S1DZ \\
\hline Primary somatosensory cortex - oral dysgranular zone & S1DZO \\
\hline Primary somatosensory cortex - forelimb region & S1FL \\
\hline Primary somatosensory cortex - hindlimb region & S1HL \\
\hline Primary somatosensory cortex - jaw region & S1J \\
\hline Primary somatosensory cortex - shoulder region & S1Sh \\
\hline Primary somatosensory cortex - trunk region & S1Tr \\
\hline Primary somatosensory cortex - upper lip region & S1ULp \\
\hline Secondary somatosensory cortex & S2 \\
\hline Substantia nigra - reticular part & SNR \\
\hline Temporal association cortex & TeA \\
\hline Thalamus & $\begin{array}{l}\text { MD, MDC, MDL, MDM, MDPL, PV, PVA, PVP, VPL, } \\
\text { VPM, Po, VL }\end{array}$ \\
\hline Visual cortex & V1, V1B, V1M, V2L, V2ML, V2MM \\
\hline
\end{tabular}

Table 1. Atlas-based network regions. All 42 unilateral rat brain atlas regions (16 sensorimotor network regions in bold) were based on 3D renderings from the Paxinos and Watson rat brain atlas. 


\section{Minimum spanning tree analysis}

For all rats' graphs $G$, the MST was calculated using the Prim-Jarník algorithm. Every graph $G$ has a set of nodes $N$, an edges set $W$ and a real valued cost ${ }^{\ell_{w}}$ assigned to each edge $w \in W$, i.e., the connectivity cost is the inversed edge weight along the connection. $\mathrm{A}$ spanning tree, defined as subset of the network nodes forming graph $G$ which connects all nodes and does not contain any cycles or loops (Jackson and Read, 2010), was computed for all unilateral (contralesional) structural network reconstructions. A minimum spanning tree $T$ minimizes the sum of the costs of its edges, ${ }^{\ell(T)}=\sum_{w \in T} \ell_{w}$ over the set of all possible MSTs on $G$ (Hidalgo et al., 2007). Group-based MST connectivity matrices were generated from the prevalence of MST connections (after binarization of individual MST network matrices) for the control and stroke groups.

The following metrics were calculated for the MSTs at nodal or network level:

i) Betweenness centrality (nodal): an often used network measure of hubness (Rubinov and Sporns, 2010), which is based on the number of shortest paths passing through a node. Betweenness centrality of a node increases with the number of those passages and is defined as $b c_{i}=\frac{1}{(n-1)(n-2)} \sum_{j \neq k, k \neq i, j \neq i}^{n} \frac{g_{j k}(i)}{g_{j k}}$ where $g_{j k}$ is the shortest path between two nodes of the MST and $g_{j k}(i)$ is the number of node paths that pass actually through node $i$.

ii) Strength (nodal): the tree node strength is a summation of all nodal connection weights (Hagmann et al., 2010; Rubinov and Sporns, 2010).

iii) Leaf number ( $N_{\text {leaf }}$ ) (network): the number of tree nodes having exactly one connection to another tree node. A higher leaf number reflects increased global network integration and efficiency (Stam et al., 2014; Tewarie et al., 2015a).

iv) Diameter (d) (network): the largest distance between any two nodes of the tree, ranging from 2 to $M=N-1$, where $M$ is the number of connections in the tree. The largest possible diameter decreases with increasing leaf number $N_{\text {leaf }}$ (i.e. improved network integration or global efficiency) (Stam et al., 2014; Tewarie et al., 2015a).

v) Eccentricity (network): the longest shortest path length between a tree node I and any other node from the tree. Eccentricity decreases when nodes become more central in the tree (Stam et al., 2014; Tewarie et al., 2015a).

vi) Kappa (network): width of degree distribution: $K=\left\langle k^{2}\right\rangle /\langle k\rangle$ (Stam et al., 2014). This measure reflects the vulnerability to hub-node damage. Networks characterized with a scale-free degree distribution (Stam and van Straaten, 2012), e.g. neural networks, have particularly high Kappa values. 


\section{Contralesional network backbone metrics versus lesion size and sensorimotor outcome}

We related network backbone characteristics to lesion size and sensorimotor deficit score at day 70 , using univariable linear regression. A p-value $<0.05$ was considered statistically significant.

\section{Software}

Network analyses, statistical modelling and generation of some figures was performed in $R$ 3.2 (http://www.r-project.org/) using the packages igraph, reshape2, network, lattice, oro.nifit, plyr, sna, Im and ggplot2.

\section{RESULTS}

\section{Post mortem anatomical and diffusion MRI data}

Post-mortem MRI ten weeks after stroke $(\mathrm{N}=8)$ displayed ischemic lesions in the subcortical and cortical MCA territory (Figure 2, top row). Mean lesion volume was $264 \pm 72 \mathrm{~mm}^{3}$. Individual lesion volumes are shown in Table 2.

We found no significant change in volumes of the contralesional white matter, gray matter or total hemisphere of stroke animals as compared to controls (Table 3 ). Furthermore contralesional white matter $F A, R D$ and $A D$ values were not significantly different from control values (Table 3).

Figure 2 (bottom row) shows representative examples of tractography-based structural connectome reconstructions of control and post-stroke rat brain. Loss of fiber connections is clearly visible in the ipsilesional hemisphere where the lesion area is characterized by complete absence of fibers. In the contralesional hemisphere of stroke animals, fiber density was slightly $(1.1 \pm 0.01 \%)$ higher than in control animals $(t=2.05, p=0.06)$.

\begin{tabular}{llll}
\hline Rat & Ischemic damage & Lesion volume $\left(\mathbf{m m}^{\mathbf{3}}\right)$ & Sensorimotor deficit score \\
\hline 1 & Subcortical + cortical & 206 & 2 \\
2 & Subcortical + cortical & 275 & 6 \\
3 & Subcortical + cortical & 100 & 1 \\
4 & Subcortical + cortical & 216 & 5 \\
5 & Subcortical + cortical & 214 & 0 \\
6 & Subcortical + cortical & 304 & 4 \\
7 & Subcortical + cortical & 216 & 4 \\
8 & Subcortical + cortical & 129 & 1 \\
\hline
\end{tabular}

Table 2. Features of individual rats at 70 days after stroke. 


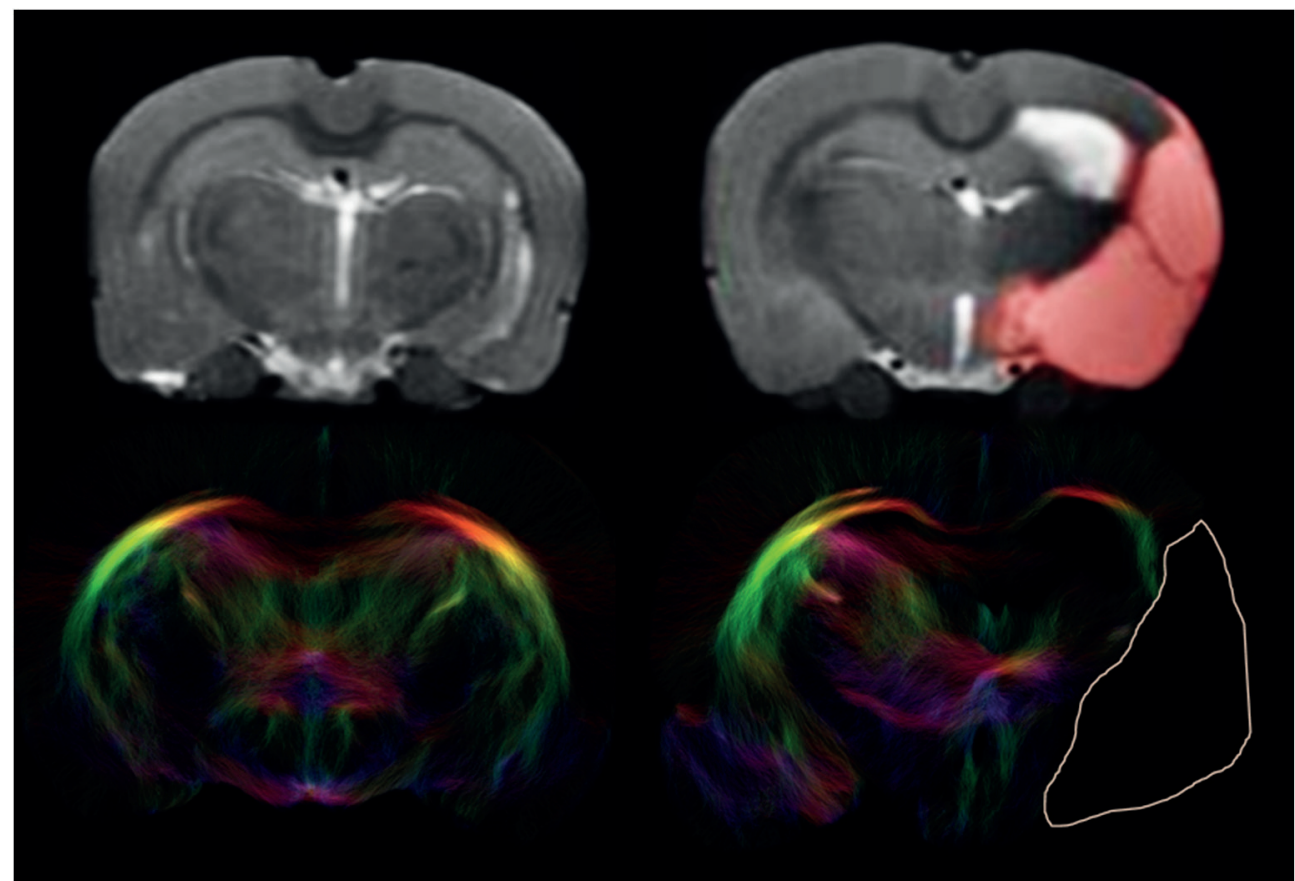

Figure 2. Anatomical images and tractograms of control and stroke rat brain. Coronal views of in vivo anatomical $\mathrm{T}_{2}$ maps (top) and corresponding post mortem diffusion-based tractograms (bottom) of a control (left) and post-stroke (right) rat brain slice ( $\mathrm{T}_{2}$-weighted and diffusion $\mathrm{MRI}$ sequences are described in the Methods section). The unilateral ischemic lesion area is depicted by a red overlay in the anatomical image and delineated by a white line in the tractogram. Loss of fiber connections is clearly visible in the ipsilesional hemisphere of stroke animals. In the contralesional hemisphere, fiber density was comparable to that in controls.

\begin{tabular}{lll}
\hline Anatomical characteristic & Controls $(\mathbf{N}=\mathbf{1 0})$ & Stroke $(\mathbf{N}=\mathbf{8})$ \\
\hline Total volume $\left(\mathrm{mm}^{3}\right)$ & $909 \pm 47$ & $890 \pm 40$ \\
Gray matter volume $\left(\mathrm{mm}^{3}\right)$ & $373 \pm 98$ & $326 \pm 70$ \\
White matter volume $\left(\mathrm{mm}^{3}\right)$ & $536 \pm 60$ & $564 \pm 38$ \\
Fractional anisotropy & $0.34 \pm 0.01$ & $0.34 \pm 0.01$ \\
Radial diffusivity $\left(\times 10^{-3} \mathrm{~mm}^{2} / \mathrm{s}\right)$ & $0.21 \pm 0.03$ & $0.22 \pm 0.02$ \\
Axial diffusivity $\left(\times 10^{-3} \mathrm{~mm}^{2} / \mathrm{s}\right)$ & $0.35 \pm 0.05$ & $0.37 \pm 0.04$ \\
\hline
\end{tabular}

Table 3. Gray and white matter characteristics for control and stroke animals.

\section{Group-based structural network and MST connectivity matrices}

Figure 3 shows group-based average connectivity matrices for the total structural network and the network backbones (MST) for the left (contralesional) hemisphere of stroke and control animals (see Figure $\mathbf{S 1}$ for representative examples of individual weighted networks and corresponding backbones). Some structural connections are highly stable (high prevalence) whereas existence of other connections varies across individual animals (low 
prevalence). The MSTs, reflecting the backbone connections, primarily consisted of connections between sensorimotor regions, such as the primary motor cortex, the secondary motor cortex, caudate putamen and the forelimb and hindlimb regions of the primary somatosensory cortex. Overall the connectivity pattern in the contralesional hemisphere of stroke animals was quite similar to its counterpart in control animals. However, subtle differences were apparent in the connectivity matrices. For example in the caudate putamen, which revealed connections with the ventral area of the secondary auditory cortex and the primary auditory cortex in the stroke group but not in the control group. Also for the backbone connections, we observed connections (e.g. between the upper lip region and jaw region of the primary somatosensory cortex or between the secondary motor cortex and piriform cortex) in the stroke group that were not apparent in controls. However, no significant differences in group-based average network backbone characteristics were found between the left, contralesional hemisphere in stroke animals and the left hemisphere in control animals (Figure S2).

\section{Nodal MST metrics}

Figure 4 (left) shows the ranking of individual node strengths measured from MSTs from the individual control and stroke animals. The caudate putamen and hippocampus were the strongest connected nodes in control as well as stroke animals. The upper lip and jaw region of the primary somatosensory cortex displayed considerable modifications, i.e. increased node strength (Figure 4) and degree (Figure S3), in stroke animals.

Ranking of individual node betweenness centralities (i.e. hubness) (Figure 4, right) showed that the caudate putamen, the hippocampus, the primary motor cortex and the secondary motor cortex were the four most significant hub-nodes, for control as well as stroke animals. Nevertheless, some regions displayed increased (e.g. the upper lip and jaw regions of the primary somatosensory cortex) or decreased betweenness centrality (e.g. the pre-limbic cortex) after stroke. 


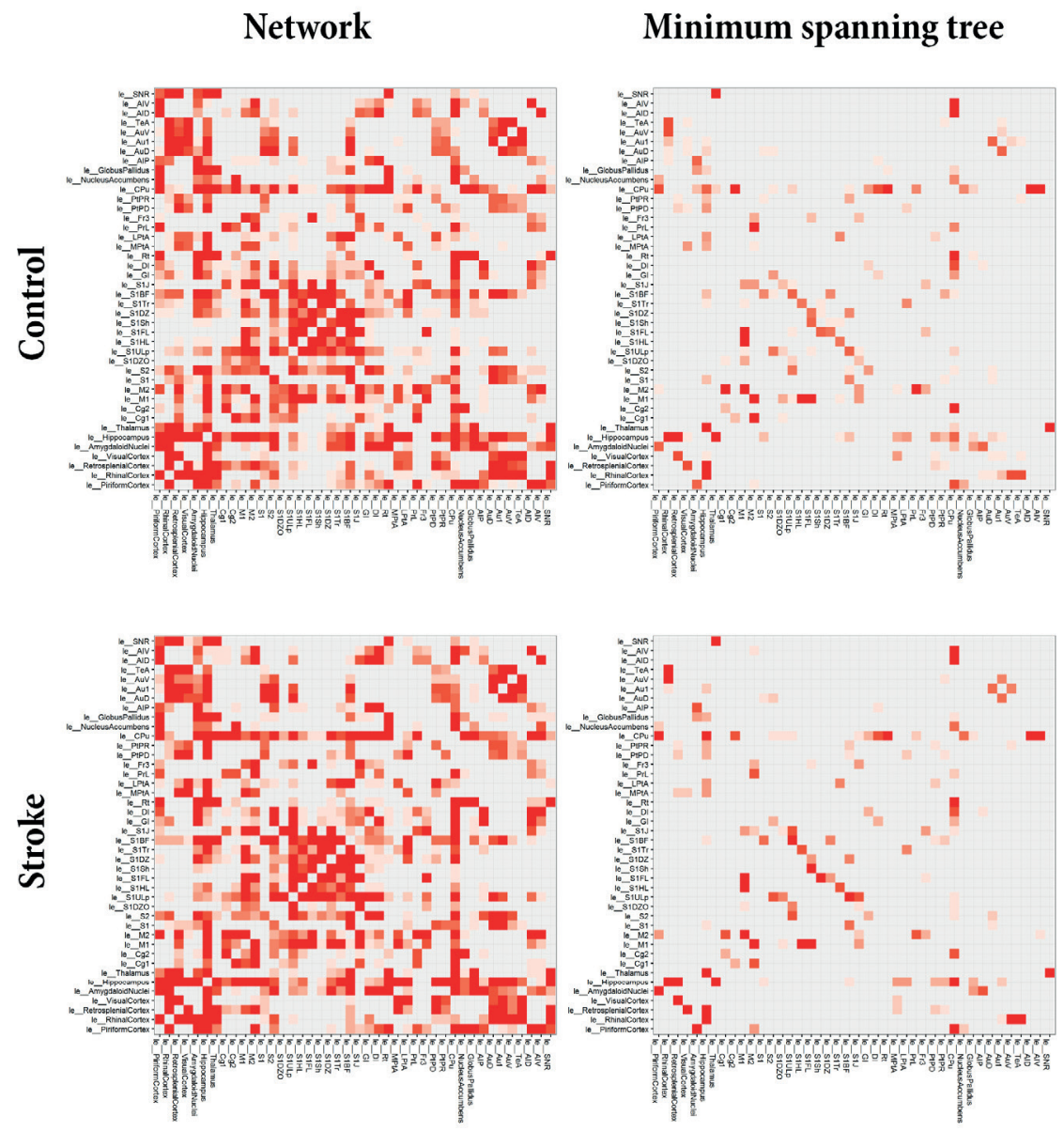

Figure 3. Average connectivity matrices for the total networks and minimum spanning tree. Average structural connectivity matrices of total networks (left) and MST backbones (right) of the left (contralesional) hemisphere for control (top) and stroke (bottom) animals. Connectivity weights are based on prevalence of connections for each group, ranging from low (white) to high (red). 'le_[Name]' indicates node in left (contra-lesional) hemisphere. The MSTs, reflecting the backbone connections, primarily consisted of connections between sensorimotor regions, such as the primary motor cortex, the secondary motor cortex, caudate putamen and the forelimb and hindlimb regions of the primary somatosensory cortex. Overall the connectivity pattern in the contralesional hemisphere of stroke animals was quite similar to its counterpart in control animals, although some subtle differences are apparent in the connectivity matrices. 

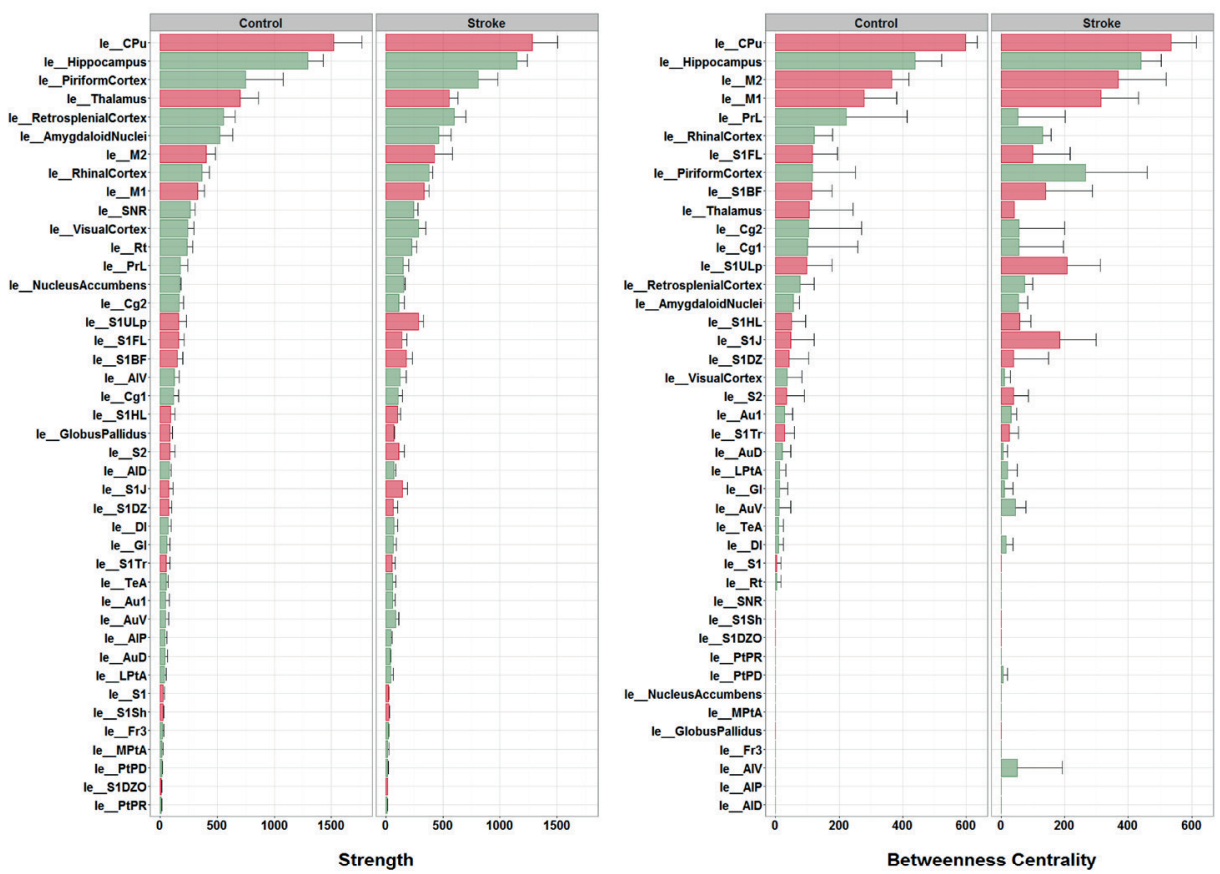

Figure 4. Node strengths and betweenness centralities from individual networks. Node strength (left) and betweenness centrality (right) (mean \pm standard deviation) -calculated from MSTs of the left (contralesional) hemisphere in individual control and stroke animals- ranked from high to low (based on control group data). 'le_[Name]' indicates brain atlas region in left (contralesional) hemisphere. Red bars represent sensorimotor regions. The caudate putamen and hippocampus were the strongest connected nodes in control as well as stroke animals. The upper lip and jaw region of the primary somatosensory cortex displayed considerable modifications, i.e. increased node strength in stroke animals. The caudate putamen, the hippocampus, the primary motor cortex and the secondary motor cortex were the four most significant hub-nodes, for control as well as stroke animals. Some regions displayed increased (e.g. the upper lip and jaw regions of the primary somatosensory cortex) or decreased betweenness centrality (e.g. the pre-limbic cortex) after stroke.

\section{Network backbone metrics versus lesion size and functional outcome}

Linear regression revealed no trends or significant association between contralesional network backbone metrics at post-stroke day 70 and lesion volume (Figure S4). Also, sensorimotor deficit score at day 70 (shown in Table 2) was not significantly correlated with MST backbone characteristics of the total structural network (Figure 5, left). However, sensorimotor deficit score was positively associated with eccentricity $(p=0.02)$ and average betweenness centrality $(p=0.08)$ of the specific MST from the contralesional sensorimotor network (Figure 5, right). 

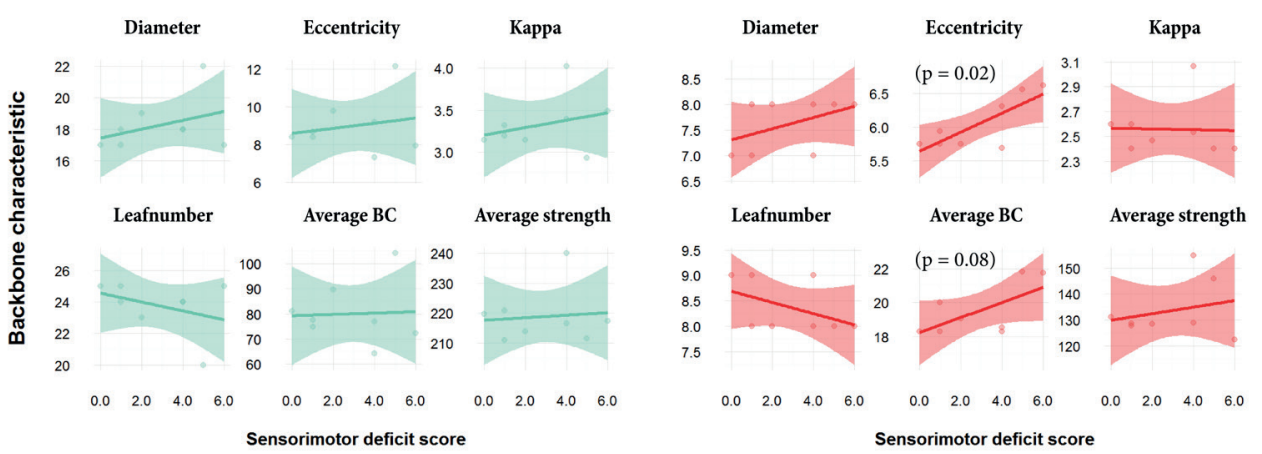

Figure 5. Contralesional backbone metrics versus sensorimotor deficit score. Linear model fits of MST metrics for the total structural network (left, green) and the sensorimotor network (right, red) in the contralesional hemisphere versus sensorimotor deficit score at day 70 after stroke (transparent bands indicate standard deviation). BC: betweenness centrality. Sensorimotor deficit score was not significantly correlated with MST backbone characteristics of the total structural network. However, sensorimotor deficit score was positively associated with eccentricity and average betweenness centrality of the specific MST from the contralesional sensorimotor network.

\section{DISCUSSION}

To characterize the configuration of structural network backbones in the contralesional hemisphere chronically after stroke we analyzed high-resolution post-mortem MRI data of rat brains collected at 70 days after unilateral stroke. Structural backbones, assessed with an unbiased network analysis approach based on MSTs, were relatively consistent across control and stroke animals, but modifications in nodal importance of specific sensorimotor network regions were apparent in post-stroke brains. Furthermore, we found that sensorimotor outcome was associated with backbone metrics related to network efficiency.

Changes in the organization of neural networks in the contralesional hemisphere after stroke may reflect remote tissue degeneration as well as remote tissue plasticity in response to focal ischemic injury (Biernaskie and Corbett, 2001; Carter et al., 2012a; Crofts et al., 2011; Dijkhuizen et al., 2012; Grefkes and Fink, 2011; Jones et al., 1996; Stroemer et al., 1995). In our study we measured an increase in connectivity strength and hubness of certain contralesional sensorimotor regions (e.g. upper lip and jaw regions of the primary somatosensory cortex) in brains from rats that were sacrificed at a plateau stage of sensorimotor recovery after stroke. These findings suggest adaptive reorganization of the contralesional sensorimotor network with recruitment of distinct sensorimotor regions, possibly through strengthening or formation of connections. This corresponds with our previous findings of enhanced in vivo functional connectivity (part of which measured in the 
same group of stroke animals (van Meer et al., 2010b)) and stronger neuroanatomical connectivity between contralesional sensory and motor regions (van Meer et al., 2010a, 2010b). In the current study, the observed increased consistency, strength and hubness of network backbone regions in stroke animals may particularly denote strengthening of existing connections. In an earlier diffusion MRI study in which network communicability was measured in chronic stroke patients, several regions with increased communicability were identified in the contralesional hemisphere (Crofts et al., 2011). Yet, contralesional regions with decreased communicability were also observed, which may be related to secondary degeneration of trans-hemispheric white matter pathways (Schaechter et al., 2010). However, diffusion MRI data from our study in rats did not reveal significant changes in white matter volume or integrity in the contralesional hemisphere.

Sensorimotor outcome at day 70 was negatively associated with eccentricity and average betweenness centrality of specifically the sensorimotor part of the contralesional network backbone (no significant correlation was found for the backbone of total contralesional network). This implies that low integration and global efficiency (i.e. high network eccentricity), and strong reliance on highly connected nodes (i.e. high average betweenness centrality) in the contralesional sensorimotor network may impede recovery of sensorimotor function after stroke, which would further emphasize the importance of efficient (modified) network functioning in the contralesional hemisphere of recovering stroke patients.

Despite the potential of network backbone analysis for reliable and robust assessment of the strongest and most important connections, we cannot draw conclusions about the contribution of other structural connections that may have played a role in network remodeling in our study. Furthermore, although our post mortem dataset offered highquality reconstruction of structural connections, it was limited to a single chronic time-point after stroke, which did not allow us to look into earlier structural network changes when functional recovery is particularly ongoing, i.e. in the first weeks after stroke. Nevertheless, our study shows that modifications in structural network backbones at meso- to macroscale level can be identified from high-resolution diffusion-based tractography in rat brain. We measured alterations in MST metrics in the contralesional hemisphere chronically after stroke, which we could relate to the degree of functional outcome.

The relative voxel size as compared to total rat brain volume $\left(8 \times 10^{-3} \mathrm{~mm}^{3} / 900 \mathrm{~mm}^{3}\right)$ in our post mortem study, is comparable to that of in vivo DTI studies in humans (8-27 $\mathrm{mm}^{3} / 1500 \times 10^{3} \mathrm{~mm}^{3}$ ). It would be valuable, for translational purposes, to assess structural network backbone configurations with DTI in stroke patients. Furthermore, longitudinal studies could help to resolve the temporal pattern of structural network alterations in relation to loss and recovery of functions. Alternative network methods, based on generative models, may be employed to further elucidate structural repair mechanisms in 
post-stroke brain at whole-connectome level. These include Bayesian exponential random graph models (Caimo and Friel, 2011; Sinke et al., 2016), or mixed-effect models (Simpson and Laurienti, 2015b) and Gibbs distribution models (La Rosa et al., 2016), which also allow comparison of networks with different densities. Insights from these studies may aid in the development and selection of new treatment strategies for patients recovering from stroke. 


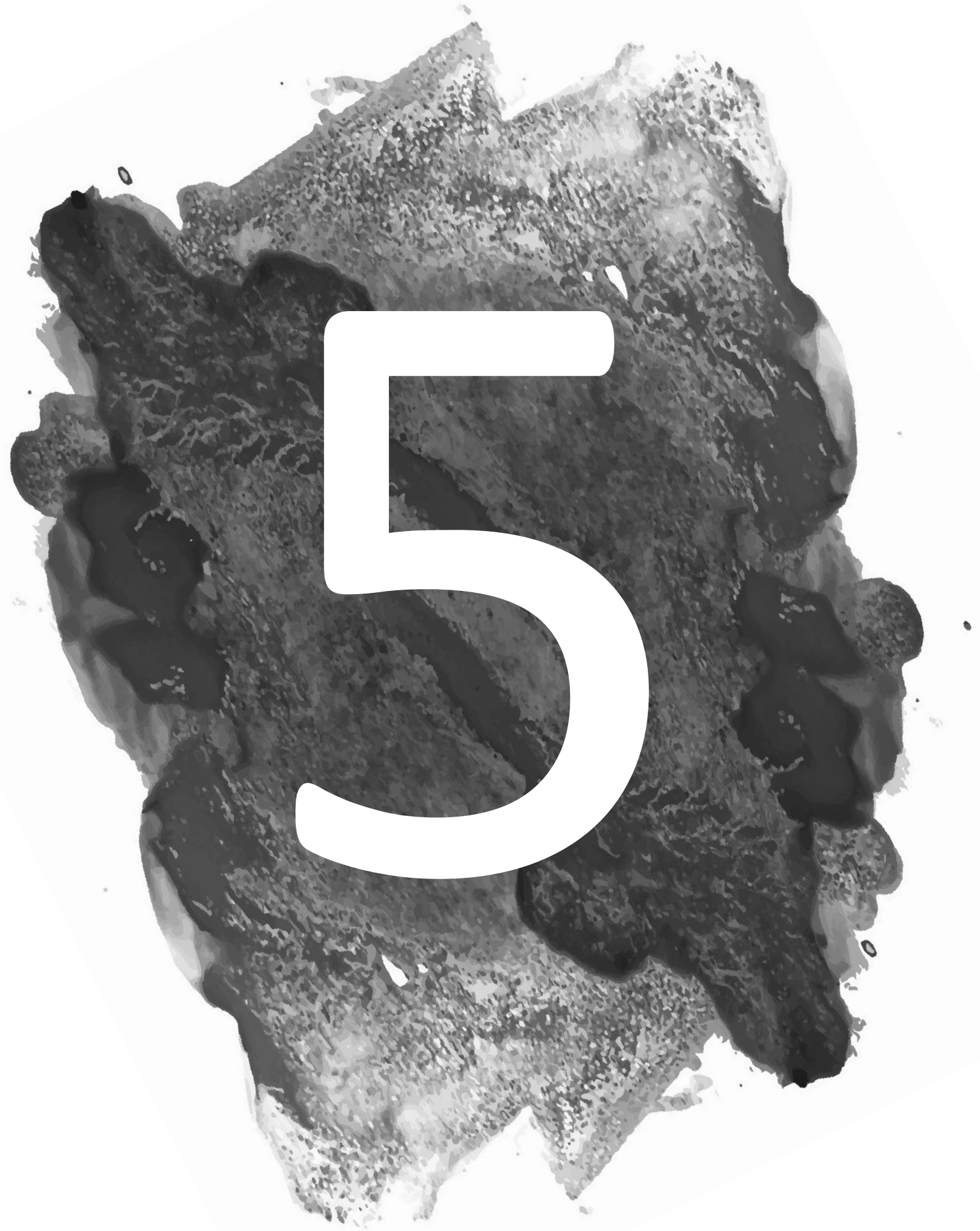




\section{Chapter 5}

Differences in Structural and Functional Networks between Young Adult and Aged Rat Brains before and after Stroke Lesion Simulations

M. Straathof ${ }^{1}$, M.R.T. Sinke ${ }^{1}$, A. van der Toorn, P.L. Weerheim, W.M. Otte \& R.M. Dijkhuizen

${ }^{1}$ Shared first author

Neurobiology of Disease (2019), Vol. 126, 23-35 


\begin{abstract}
Neural network changes during aging may contribute to vulnerability and resilience to brain lesions in age-related neurological disorders, such as stroke. However, the relationship between age-related neural network features and stroke outcome is unknown. Therefore, we assessed structural and functional network status in young adult and aged rat brain, and measured the effects of simulated stroke lesions. Eleven rats underwent diffusion-weighted MRI and resting-state functional MRI at young adult age (post-natal day 88 ) and old age (between post-natal day 760 and 880). Structural and functional brain network features were calculated from graph-based network analysis. We performed three lesion simulations based on the brain injury pattern in frequently applied rodent stroke models, i.e. a small cortical lesion, a subcortical lesion, or a large cortical plus subcortical lesion, for which we computationally removed the involved network regions. Global network characteristics, i.e. integration and segregation, were not significantly different between the two age groups. However, we detected local differences in structural and functional networks between young adult and old rats, mainly reflected by shifts of hub regions. Stroke lesion simulations induced significant global and local network changes, characterized by lower efficiency and shifts of hub regions in structural and functional networks, which was most evident after a large cortical plus subcortical lesion. Functional and structural hub region shifts after lesion simulations differed between young adult and aged rats. Our lesion simulation study demonstrates that age-dependent brain network status affects structural and functional network reorganization after stroke, particularly involving hub shifts, which may influence functional outcome. Computational lesion studies offer a cheap and simple alternative to empirical studies and can complement or guide more complicated experimental studies in animal models and patients.
\end{abstract}




\section{INTRODUCTION}

Stroke - i.e. a sudden loss of blood flow to the brain - is one of the main causes of longterm disability in adults, and affects almost 17 million people worldwide per year (Feigin et al., 2014). Despite the significant functional consequences, many patients show (partial) recovery of sensorimotor and cognitive functions during the weeks and months following stroke, which may be related to reorganization of surviving networks in the brain (Cramer, 2008; Grefkes and Fink, 2011; Jiang et al., 2013; Jones, 2017; Murphy and Corbett, 2009). Post-stroke brain remodeling occurs at different levels and locations, i.e. from micro- (e.g. synaptic plasticity) to macro-scale (e.g. cortical remapping) (Biernaskie and Corbett, 2001; Jones et al., 1996; Stroemer et al., 1995) and from peri- to contralesional sites (Cai et al., 2016; Crofts et al., 2011; Dacosta-Aguayo et al., 2014; Granziera et al., 2012; Gratton et al., 2013; Johansen-Berg et al., 2010; Schaechter et al., 2010), respectively. These insights have led to the notion that assessment of neural networks at whole-brain level is critical for optimal understanding of the functional consequences of stroke (Carter et al., 2012a; Grefkes and Fink, 2011; Rehme and Grefkes, 2013).

Brain networks consist of spatially distributed regions that are connected and interacting with each other at micro-, meso- and macro-scales (Bassett and Sporns, 2017; Bullmore and Sporns, 2009). Modern network science describes the brain as a collection of nodes (e.g. individual neurons, neuronal clusters or functional brain regions) and edges or ties (e.g. structural or functional connections between nodes). A healthy brain's network topology is described by an optimal balance between integration (i.e. global efficiency) and segregation (i.e. local specialization) of neural signaling, characterized by small-world organization, modularity and a 'rich club' of highly connected hub regions (Bassett and Bullmore, 2009; Bassett and Sporns, 2017; Bullmore and Sporns, 2009; Sporns, 2010). These topological characteristics have been found across species (van den Heuvel et al., 2015, $2016 a, 2016 b)$ and deviation from optimal organization has been observed in relation to aging, brain dysfunction and cerebral injury, including stroke (Bassett and Bullmore, 2009; Bullmore and Sporns, 2009; van Meer et al., 2012; Sporns, 2010; Stam, 2014). Moreover, network changes during aging may contribute to vulnerability and resilience to brain lesions in age-related neurological disorders, such as stroke. However, the relationship between age-related neural network features and stroke is largely unknown. Systematic studies on this relationship in stroke patients are complicated and may be more straightforwardly conducted in laboratory rodents that age relatively fast and can be scanned serially in a fairly short time-frame.

Experimental animal stroke studies are generally performed in young adult rodents, even though the risk and prevalence of human stroke is higher at older age. Recently, this age discrepancy between animal stroke models and human stroke patients has been put forward as one of the causes of poor bench-to-bedside translation (Dirnagl, 2016). The age 
at which stroke is induced is known to influence stroke outcome and response to therapies in experimental models (Herson and Traystman, 2014; Liang et al., 2016; Liu et al., 2009). In the current study, we aimed to identify differences in brain networks of young adult and aged rats as well as their susceptibility and resilience to stroke lesions. We used data from rats that were longitudinally scanned with MRI from early adulthood to old age. The imaging protocol included resting-state functional MRI (resting-state $\mathrm{fMRI}$ ) and diffusion MRI, which allowed assessment of large-scale functional and structural neural network status under healthy conditions. We simulated lesions in brain areas that are typically affected in different rodent stroke models, i.e. cortical photothrombosis (Watson et al., 1985), short transient middle cerebral artery occlusion (MCAO), and permanent or long transient MCAO (Garcia et al., 1995; Li et al., 1995). In addition, we simulated lesions in a single hub region and in a single non-hub region to measure their effect on the global network characteristics. We chose regions that are normally not affected in the abovementioned stroke models. We hypothesized that local and global network characteristics alter in relation to lesion extent, and that this relationship differs between young adult and aged rats.

\section{METHODS}

\section{Animals}

Twenty healthy male Wistar rats (Harlan, Horst, The Netherlands) were housed in groups of four rats per cage. The rats had ad libitum access to food and water, were housed with a light/dark cycle of $12 \mathrm{~h}$ (lights on at 7:00 AM), and temperature was controlled between 22 and $24^{\circ} \mathrm{C}$. All experiments were approved by the committee for Animal Experiments of the University Medical Center Utrecht, The Netherlands (protocol number 2010.I.10.228), and were performed in accordance with the guidelines of the European Communities council directive.

MRI was done at multiple time-points during the lifespan of the rats, until their natural death. We analyzed data from eleven animals that were scanned during early adulthood (postnatal day 88), i.e. comparable to the age of rats in most preclinical stroke studies, as well as at old age (postnatal day 760 or 880 ), i.e. representative of the age during which clinical stroke is prevalent. Scans from animals that died before this age were not included in the analyses.

\section{MRI acquisition}

MRI experiments were conducted on a $4.7 T$ horizontal bore MR system. We used a homebuilt Helmholtz volume coil (90 mm diameter) and an inductively coupled surface coil (25 mm diameter) for signal excitation and detection, respectively. Rats were anesthetized with $4 \%$ isoflurane and endotracheally intubated for mechanical ventilation (TOPO, Kent Scientific, Torrington, CT, USA) with $1-2 \%$ isoflurane in a mixture of air with $30 \% \mathrm{O}_{2}(55$ 
breaths per minute). Rats were subsequently immobilized in a specially designed MRcompatible stereo-tactic holder, including earplugs and a tooth holder. During MRI, endtidal $\mathrm{CO}_{2}$ as well as blood oxygen saturation and heart rate were continuously monitored with a capnograph (Multinex 4200, Datascope Corporation, Paramus, NJ, USA) and pulse oximeter (8600V, Nonin Medical, Plymouth, MN, USA), respectively. Body temperature was maintained at $37.0 \pm 0.5^{\circ} \mathrm{C}$.

For resting-state $\mathrm{fMRI}, \mathrm{T}_{2}{ }^{*}$-weighted blood oxygenation level-dependent (BOLD) images were acquired under $1.0 \%$ isoflurane anesthesia, with a ventilation-triggered single-shot 3D gradient-echo echo planar imaging (EPI) sequence (repetition time (TR) / echo time (TE) = 32 / $19 \mathrm{~ms}$ (effective TR = $1.024 \mathrm{~s}$ ); $12^{\circ}$ pulse angle; field-of-view (FOV) $=32 \times 24 \times 12 \mathrm{~mm}^{3}$; $64 \times 48 \times 32$ acquisition matrix; $0.5 \times 0.5 \times 0.5 \mathrm{~mm}^{3}$ voxels; 600 BOLD images in approximately 10 minutes). Diffusion MRI was executed under $1.5-2.0 \%$ isoflurane with a 2D 5-shot EPI sequence (TR / TE = $1750 / 28.52 \mathrm{~ms} ; 4$ bo images, 4 b-values (650, 1285, 1919 and $\left.2518 \mathrm{~s} / \mathrm{mm}^{2}\right) ; \delta / \Delta=5 / 10 \mathrm{~ms} ; 30$ directions per b-value, three averages; $128 \times 128$ acquisition matrix; $0.195 \times 0.195 \mathrm{~mm}^{2}$ voxels; 19 1.0-mm slices). In addition, anatomical images were acquired with a 3D gradient-echo sequence (TR / TE $=6 / 2.25 \mathrm{~ms} ; 40^{\circ}$ flip angle; 4 averages; FOV $=40 \times 25 \times 20 \mathrm{~mm}^{3} ; 160 \times 100 \times 80$ matrix; $0.25 \times 0.25 \times 0.25 \mathrm{~mm}^{3}$ voxels).

\section{Image processing}

\section{Resting-state functional connectivity}

Resting-state functional MR images were processed with FSL 5.0 (Jenkinson et al., 2012), unless otherwise stated. Preprocessing steps of the resting-state fMRI scans included removal of the first 10 images to reach a steady state, motion-correction with MCFLIRT (Jenkinson et al., 2002) and brain-extraction with BET (Smith, 2002). Motion-correction parameters were used as regressors for the resting-state signal (no linear detrending and global mean regression were performed). Low-frequency BOLD fluctuations were obtained by applying temporal filtering between 0.01 and $0.1 \mathrm{~Hz}$ in AFNI (Cox, 1996). We calculated Fisher's Z-transformed correlation coefficients to measure inter- and intrahemispheric functional connectivity between regions-of-interest (ROIs) (see below). Individual functional connectivity matrices were divided by their own mean to correct for individual differences in mean functional connectivity strength. This procedure is based on a normalization step in the analysis of structural networks described by Van den Heuvel et al. (2010). They reported normalization by dividing all network edge values by the maximum network edge value. However, since many functional connectivity networks in our study were skewed towards lower correlation values with high value outliers (see Figure S1), we normalized functional network values with the mean rather than the maximum value. For comparison, we performed a sensitivity analysis with the maximum value as normalization 
factor and data are presented in Figure S2-S5. Results were highly similar between mean and maximum normalization procedures.

\section{Diffusion-based structural connectivity}

Diffusion-weighted images were preprocessed with FSL 5.0 (Jenkinson et al., 2012) and MRtrix3 (Tournier et al., 2012). Motion and eddy current corrections were done with dwipreproc (MRtrix3), which uses FSL tools (Andersson and Sotiropoulos, 2016). Calculation of diffusion parameters, i.e. fractional anisotropy (FA), mean diffusivity (MD), axial diffusivity $(A D)$ and radial diffusivity $(R D)$, was done with dtifit in FSL.

Further processing, tractography and connectome reconstruction were done in MRtrix3. We first determined multi-shell response functions for white matter (WM), gray matter (GM) and cerebral spinal fluid (CSF) using dwi2response and custom-made WM, GM and CSF masks. Multi-shell multi-tissue constrained spherical deconvolution (CSD) (Jeurissen et al., 2014) was performed to generate WM, GM and CSF volume fraction maps, and to obtain fiber orientation distribution (FOD) maps for WM, GM and CSF separately, of which the WM FOD map was used for tractography.

We performed CSD tractography using the iFOD2 algorithm (Tournier et al., 2010), with dynamic seeding over the WM FOD map, a step-size of $0.1 \mathrm{~mm}$, an angle threshold of $45^{\circ}$ and an FOD amplitude threshold of 0.2 , thereby generating 1 million tracts over the entire brain. After whole-brain tractography we used Spherical deconvolution Informed Filtering of Tractograms (SIFT) to improve the accuracy of the reconstructed whole-brain connectome by fitting and optimizing tracts at whole-brain level to the underlying diffusionweighted images, and by removing (i.e. filtering) inappropriate tracts from the connectome (Smith et al., 2013, 2015a).

Whole-brain structural networks were constructed by matching the filtered tracts with ROIs in subject space. Two regions were considered connected if one or more tracts traversed - or had their endpoints in - both regions. The streamline count after SIFT was used as measure of structural connectivity strength.

\section{Regions-of-interest}

After preprocessing, resting-state $\mathrm{fMRI}$ and diffusion $\mathrm{MRI}$ images were linearly and nonlinearly registered to a reference rat brain using FLIRT and FNIRT (Andersson et al., 2007; Jenkinson and Smith, 2001), respectively. The reference rat brain was aligned with a custombuilt 3D reconstruction of the Paxinos and Watson rat brain atlas (Majka et al., 2012; Paxinos and Watson, 2005). We included 88 cortical and subcortical regions from the atlas, covering most of the rat brain, with sufficient assurance of spatial alignment (i.e. inclusion of at least 8 voxels per ROI in the resting-state scans). Subsequently, all ROls were backprojected in subject space, where further analyses were performed. 


\begin{tabular}{|c|c|}
\hline Atlas description & Paxinos and Watson atlas labels \\
\hline Left and right agranular insular cortex - dorsal part & AID \\
\hline Left and right agranular insular cortex - posterior part & AIP \\
\hline Left and right agranular insular cortex - ventral part & AIV \\
\hline Left and right primary auditory cortex & Au1 \\
\hline Left and right secondary auditory cortex - dorsal area & AuD \\
\hline Left and right secondary auditory cortex - ventral area & AuV \\
\hline Left and right cingulate cortex - area 1 & Cg1 \\
\hline Left and right cingulate cortex - area 2 & $\mathrm{Cg} 2$ \\
\hline Left and right dysgranular insular cortex & DI \\
\hline Left and right dorsolateral entorhinal cortex & DLEnt \\
\hline Left and right ectorhinal cortex & Ect \\
\hline Left and right frontal cortex - area 3 & $\mathrm{Fr} 3$ \\
\hline Left and right frontal association cortex & FrA \\
\hline Left and right granular insular cortex & $\mathrm{Gl}$ \\
\hline Left and right lateral orbital cortex & LO \\
\hline Left and right lateral parietal association cortex & LPtA \\
\hline Left and right primary motor cortex & M1 \\
\hline Left and right secondary motor cortex & M2 \\
\hline Left and right medial parietal association cortex & MPtA \\
\hline Left and right perirhinal cortex & Prh \\
\hline Left and right retrosplenial dorsal & RSD \\
\hline Left and right retrosplenial granular cortex a region & RSGa \\
\hline Left and right retrosplenial granular cortex $b$ region & RSGb \\
\hline Left and right primary somatosensory cortex - barrel field & S1BF \\
\hline Left and right primary somatosensory cortex - dysgranular zone & S1DZ \\
\hline Left and right primary somatosensory cortex - forelimb region & S1FL \\
\hline Left and right primary somatosensory cortex - hindlimb region & S1HL \\
\hline Left and right primary somatosensory cortex - jaw region & S1J \\
\hline Left and right primary somatosensory cortex - trunk region & S1Tr \\
\hline Left and right primary somatosensory cortex - upper lip region & S1ULp \\
\hline Left and right secondary somatosensory cortex & S2 \\
\hline Left and right temporal association cortex & TeA \\
\hline Left and right primary visual cortex & V1 \\
\hline Left and right primary visual cortex - binocular area & V1B \\
\hline Left and right primary visual cortex - monocular area & $\mathrm{V} 1 \mathrm{M}$ \\
\hline Left and right secondary visual cortex - lateral area & V2L \\
\hline Left and right secondary visual cortex - mediolateral area & V2ML \\
\hline Left and right secondary visual cortex - mediomedial area & V2MM \\
\hline Left and right ventral orbital cortex & vo \\
\hline Left and right thalamus & $\mathrm{T}$ \\
\hline Left and right globus pallidus & GP \\
\hline Left and right caudate putamen & $\mathrm{CPu}$ \\
\hline Left and right accumbens nucleus & Acc \\
\hline Left and right hippocampus & Hipp \\
\hline
\end{tabular}

Table 1. Atlas-based network regions. Included regions-of-interest for resting-state $\mathrm{fMRI}$ and diffusion MRI analyses.

\section{Graph-based network analysis}

To determine the effects of lesion simulations on global and local network characteristics of structural and functional brain networks, we used graph analyses (Bullmore and Sporns, 2009). These graph analyses were performed on individual weighted networks, for restingstate $\mathrm{fMRI}$ and diffusion $\mathrm{MRI}$ networks separately. A weighted graph $\mathrm{G}=(\mathrm{V}, \mathrm{W})$ was 
constructed with $\mathrm{V}$ as the collection of all included regions $\mathrm{N}$, and $\mathrm{W}$ as the collection of all edge weights $\mathrm{w}$. Self-connections were excluded, and negative edge weights in the functional weighted graphs were set to 0 . The Fisher's Z-transformed correlation coefficient was used as functional edge weight, while the SIFT-corrected streamline count was used as structural edge weight. To characterize brain networks, we calculated global as well as local network parameters separately.

\section{Global parameters}

To characterize the network topology we measured several global network parameters: the weighted-undirected clustering coefficient (C) (Fagiolo, 2007) as a measure of segregation, the weighted shortest path length (L) (Stam et al., 2009), as a measure of global efficiency or integration, and the small-worldness (Humphries and Gurney, 2008). All global parameters were calculated for structural and resting-state functional networks.

- Clustering coefficient (C): The weighted clustering coefficient provides a measure of the degree to which the nearest neighbors of each node are directly connected to each other. It sums the weights of the connections that exist between the nearest neighbors, divided by the potential maximum number of connections in triplets of nodes. We first calculated the weighted $C$ for each node $i$ in the entire graph:

$$
C_{i}=\frac{\sum_{j \neq i} \sum_{h \neq(i, j)} w_{i j}^{\frac{1}{3}} w_{i h}^{\frac{1}{3}} w_{j h}^{\frac{1}{3}}}{d_{i}\left(d_{i}-1\right)}
$$

In which $d_{i}$ is the number of connections of node $i$. This equation considers weights of all edges in a triplet and excludes weights that are not participating in a triplet. Subsequently, the weighted $\mathrm{C}$ was determined by taking the mean of the local clustering coefficients over all the nodes in the network:

$$
C=N^{-1} \sum_{i=1}^{N} C_{i}
$$

- Weighted shortest path length (L): The weighted shortest path length provides a measure of the average minimal distance between a node and all the other nodes in the network. This is measured as the inverse of the weights of the connections that have to be crossed to go from one node to another. We first calculated the weighted path length $\left(\mathrm{I}_{\mathrm{ij}}\right)$ for each pair of nodes ( $\mathrm{i}$ and $\mathrm{j}$ ) in the entire graph using Dijkstra's algorithm for weighted graphs (Dijkstra, 1959), by taking the minimum sum of the inverse weights $\left(d_{i j}\right)$ to travel between node $\mathrm{i}$ and $\mathrm{j}$ :

$l_{i j}^{w}=\min _{i \leftrightarrow j}\left(\operatorname{sum}\left(d_{i j}\right)\right)$ and $d_{i j}=1 / w_{i j}$ 
To handle disconnected edges, characterized by an infinite path length, we used the harmonic mean $(1 / \infty \rightarrow 0)$. The harmonic mean takes the reciprocal of the mean of the reciprocals (Newman, 2003):

$$
L=N(N-1) / \sum_{i=1, j \neq i}^{N} \frac{1}{l_{i j}^{W}}
$$

- For each functional and structural network, C and L were normalized based on 10 randomly rewired surrogate networks (Maslov and Sneppen, 2002). The normalized weighted clustering $(\gamma)$ and path length $(\lambda)$ were defined as:

$$
\gamma=\frac{C}{C_{\text {random }}} \quad \lambda=\frac{L}{L_{\text {random }}}
$$

- Small-worldness was calculated as a measure of the optimal balance between segregation (clustering) and integration (path length), by $\gamma / \lambda$, according to Humphries and Gurney (Humphries and Gurney, 2008).

\section{Local parameters}

To investigate local (i.e. nodal) characteristics of the network, we identified most important nodes, i.e. the hubs, in the network, by quantifying the strength (weighted version of degree) (Barrat et al., 2004) and betweenness centrality (Brandes, 2011; Freeman, 1977) for each node separately.

- Strength (S): The connection strength of a node is the sum of all the edge weights connected to that node. This provides a measure of the total nodal connectivity (strength). We calculated the strength of each node in the entire graph:

$$
S_{i}=\sum_{j=1}^{N} w_{i j}
$$

- $\quad$ Betweenness centrality $\left(C_{B}\right)$ : The weighted betweenness centrality of a node provides a measure of the importance of the node in network connectivity. It measures the degree to which a node lies on the shortest path between two other nodes. We calculated the betweenness centrality for each node in the entire graph:

$$
C_{B}(v)=\sum_{s \neq v \neq t \in V} \frac{\sigma_{s t}(v)}{\sigma_{s t}}
$$

In this formula, $\sigma_{s t}(v)$ is the number of shortest paths from $s$ to $t$ in which node $v$ is partaking.

- Hub nodes, or hub regions, are regions in the brain with a central position, which play a crucial role in network communication. Hub regions can be defined by different metrics. They generally have a low clustering coefficient and shortest path length, and a high average strength and betweenness centrality (Bullmore and Sporns, 2009; Sporns et al., 2007). Therefore, we determined the hubs based on these four characteristics, 
as previously described (van den Heuvel et al., 2010). First, we averaged these four characteristics for each region over all individual rats at each time-point separately. Subsequently, we determined which regions belonged to the top $20 \%$ for each of the four characteristics separately (the regions with the $20 \%$ lowest clustering coefficients or path lengths, and/or the $20 \%$ highest strengths or betweenness centralities). When the node belonged to the top $20 \%$ for a category, the hub score for that category was one. Regions could get a hub score between 0 and 4 , and hub regions were identified as regions with a hub score of 2 or higher.

\section{Lesion simulations}

To quantify effects of lesion simulations in young adult and aged rats, we simulated three different stroke lesion types that are typically observed in frequently applied rat stroke models (Fluri et al., 2015): small cortical lesions, subcortical lesions and large cortical plus subcortical lesions (see Figure 1). Lesions were simulated by elimination of edges from the nodes that were selected as part of the lesion (i.e. structural and functional connections were set to 0). Subsequently, network parameters were calculated as described above, for each type of simulated stroke lesion in young adult and aged rats.

\section{Small cortical lesion}

A focal cortical lesion is a hallmark of the photothrombotic stroke model, which involves systemic injection of a photosensitive dye (Rose-Bengal) followed by focal illumination of the cortex through the intact skull (Watson et al., 1985). In the rat photothrombotic stroke model, lesion induction is often targeted to the forelimb area of the somatosensory cortex (S1FL). Therefore we modeled photothrombotic stroke by eliminating S1FL nodes in our functional and structural networks.

\section{Subcortical lesion}

Probably the most frequently applied rat stroke model involves unilateral occlusion of the middle cerebral artery (MCA) with an intraluminal filament (Longa et al., 1989). Short temporary (30-60 $\mathrm{min}$ ) MCA occlusion usually results in subcortical damage in the caudate putamen $(\mathrm{CPu})$, modeled in our study by eliminating the $\mathrm{CPu}$ node in functional and structural networks.

\section{Large cortical plus subcortical lesion}

Longer (60-120 $\mathrm{min}$ ) or permanent MCA occlusion induces extensive cortical and subcortical damage. We simulated these large lesions by eliminating the CPu node and nodes representing all sub-regions of the primary somatosensory cortex (S1) (i.e. the forelimb region $(\mathrm{S} 1 \mathrm{FL})$ and hindlimb region $(\mathrm{S} 1 \mathrm{HL})$, jaw region $(\mathrm{S} 1 \mathrm{~J})$, upperlip region (S1ULp), barrel 
field region (S1BF), trunk region (S1Tr) and the dysgranular zone of S1 (S1DZ)), the secondary somatosensory cortex (S2), and the insular cortex (i.e. AID, AIP, AIV, DI and GI).

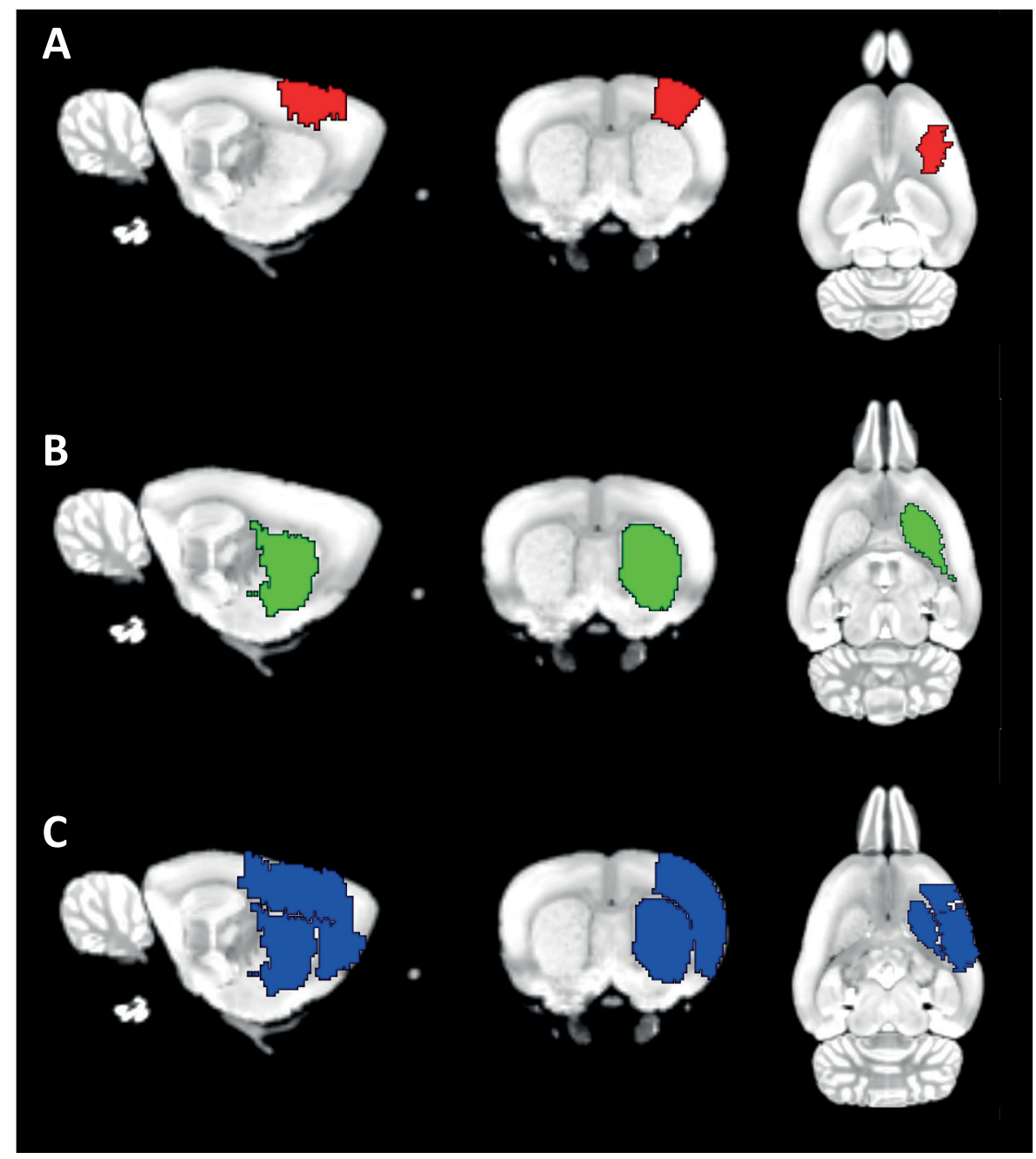

Figure 1. Lesion simulations. Regions overlaid on a sagittal (left), coronal (middle) and transversal (right) rat brain slice indicate typical lesion areas after cortical photothrombotic stroke (A (red)), and short (B (green)) or long (C (blue)) middle cerebral artery occlusion. Simulated lesion areas involved multiple slices throughout the brain, but are here displayed on a single slice in each plane for illustration purposes.

\section{Lesions in a single hub or non-hub region}

We simulated lesions in two additional areas: (1) the right hippocampus, a hub region in both structural and functional networks in young adult and aged rats, and (2) the right medial parietal association cortex, a non-hub region in all networks. 


\section{Statistical analyses}

To statistically determine whether global and local network characteristics differed between healthy young adult and aged rats, we performed paired t-testing for each global and nodal parameter. Because local characteristics were determined for 88 regions-ofinterest, we corrected the nodal analyses for multiple testing using a false-discovery rate (FDR) correction (Benjamini and Hochberg, 1995). Comparisons with p-values lower than 0.05 after FDR correction were considered statistically significantly different. To test for homotopic symmetrical hubs, i.e. whether homotopic regions in the left and right hemispheres both belonged to the hub regions, we calculated the Dice index (Yin and Yasuda, 2006) for homotopic hub regions in young adult and aged rats separately.

To assess the effect of simulated stroke lesions, we calculated the difference value for each global or nodal characteristic between the healthy condition and lesion simulation: value $_{\Delta}=$ value $_{\text {lesioned network }}$ - value healthy network. To measure the effect of a lesion on network characteristics, we first tested whether the network parameters changed significantly compared to the healthy condition with a one sample t-test for each simulated lesion type at each age separately. In addition, we compared the effects of different stroke lesion simulations on the network parameters with an ANOVA for each age separately. Secondly, we evaluated for each simulated lesion type, whether the lesion-induced change in network parameters differed between young adult and aged rats using a paired t-test. In addition, we calculated the Dice index for the hub regions in structural and functional networks before (healthy condition) and after each type of simulated lesion for young adult and aged rats separately.

We constructed $95 \%$ confidence intervals for the Dice indices by means of bootstrapping. This involves repeated calculation of the Dice indices on 10.000 resampled sets, with replacement (Bland and Altman, 2015). Subsequently, we used bootstrapping to construct $95 \%$ confidence interval of delta Dice indices. To test whether the homotopic organization of hubs, as well as hub shifts after the simulated lesions, were significantly different between young and old rats we determined p-values from the $95 \%$ confidence intervals (Altman and Bland, 2011).

\section{RESULTS}

Stable global structural and functional network features between young adulthood and old age.

Figure 2 shows quantitative results from the graph-based whole-brain network analyses of functional connectivity and structural tractography data from young adult and aged rats. For structural (Figure 2A) as well as resting-state functional networks (Figure 2B), we found no significant differences in global network measures, i.e. clustering coefficient, path length and small-worldness, between young adult and aged rats. 


\section{Higher nodal strength in structural network of aged rats}

To investigate local network features in the rat brain, we calculated the nodal strength and betweenness centrality for each region-of-interest in the structural and resting-state functional networks. We found statistically significant differences in node strength between young adult rats and aged rats for several regions in the structural network, which are listed Table 2. In all these regions node strength - and betweenness centrality for the right frontal area 3 , left cingulum area 2 and left lateral orbital cortex - was increased at old age, except for the left dysgranular insular cortex, which showed decreased node strength.

In contrast, there were no statistically significant differences in strength or betweenness centrality of the functional network nodes between young adult and aged rats.

\begin{tabular}{|c|c|c|c|}
\hline Region of interest & Nodal network feature & $\Delta$ Percentage & FDR corrected P-value \\
\hline Left Acc & Strength & 80 & $<0.01$ \\
\hline Right Acc & Strength & 100 & $<0.01$ \\
\hline Left AID & Strength & 111 & $<0.01$ \\
\hline Right AID & Strength & 111 & $<0.01$ \\
\hline Right AIP & Strength & 88 & 0.02 \\
\hline Left AIV & Strength & 167 & $<0.001$ \\
\hline Right AIV & Strength & 184 & $<0.001$ \\
\hline Right AuV & Strength & 75 & 0.04 \\
\hline Left Cg1 & Strength & 119 & $<0.001$ \\
\hline Right Cg1 & Strength & 85 & $<0.01$ \\
\hline Left Cg2 & Betweenness & 237 & 0.04 \\
\hline Left Cg2 & Strength & 65 & $<0.01$ \\
\hline Right Cg2 & Strength & 58 & $<0.01$ \\
\hline Left CPu & Strength & 45 & $<0.01$ \\
\hline Right $\mathrm{CPu}$ & Strength & 55 & $<0.01$ \\
\hline Left DI & Betweenness & -33 & 0.04 \\
\hline Right DI & Strength & 88 & $<0.01$ \\
\hline Left Fr3 & Strength & 147 & $<0.001$ \\
\hline Right Fr3 & Betweenness & 84 & 0.02 \\
\hline Left FrA & Strength & 1088 & $<0.01$ \\
\hline Left GP & Strength & 43 & 0.02 \\
\hline Right GP & Strength & 74 & $<0.01$ \\
\hline Left LO & Betweenness & 172 & 0.03 \\
\hline Left LO & Strength & 293 & $<0.001$ \\
\hline Right LO & Strength & 193 & $<0.001$ \\
\hline Left M1 & Strength & 65 & $<0.01$ \\
\hline Right M1 & Strength & 105 & $<0.001$ \\
\hline Left M2 & Strength & 89 & $<0.01$ \\
\hline Right M2 & Strength & 125 & $<0.01$ \\
\hline Right MptA & Strength & 82 & 0.02 \\
\hline Right Prh & Strength & 50 & 0.02 \\
\hline Right RSGb & Strength & 44 & 0.03 \\
\hline Right S1BFa & Strength & 35 & $<0.01$ \\
\hline Right S1J & Strength & 60 & 0.04 \\
\hline Left T & Strength & 55 & $<0.01$ \\
\hline Right T & Strength & 78 & $<0.01$ \\
\hline Left V1 & Strength & 60 & 0.02 \\
\hline Left V2ML & Strength & 142 & 0.04 \\
\hline Right V2MM & Strength & 102 & 0.04 \\
\hline Left Vo & Strength & 181 & $<0.001$ \\
\hline
\end{tabular}

Table 2. Regions with significantly altered nodal network measures in aged rats compared to young adult rats. 


\section{A) Global structural network}
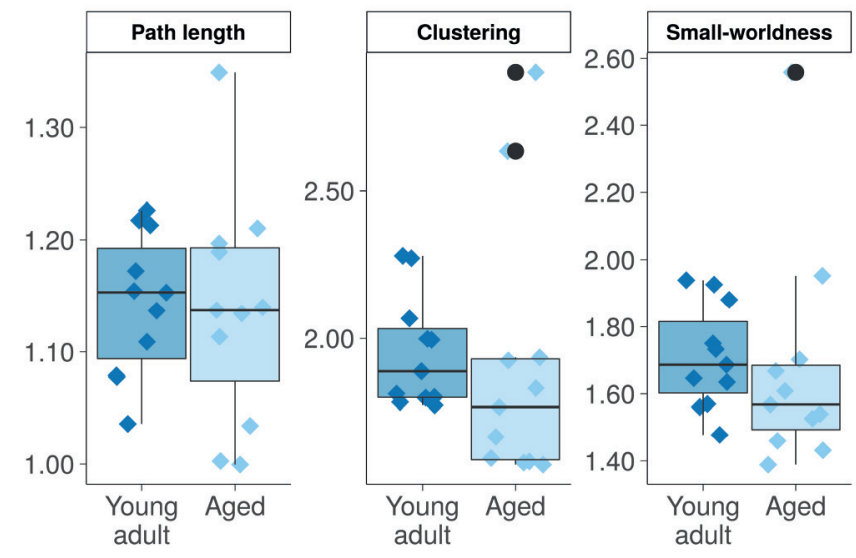

\section{B) Global functional network}
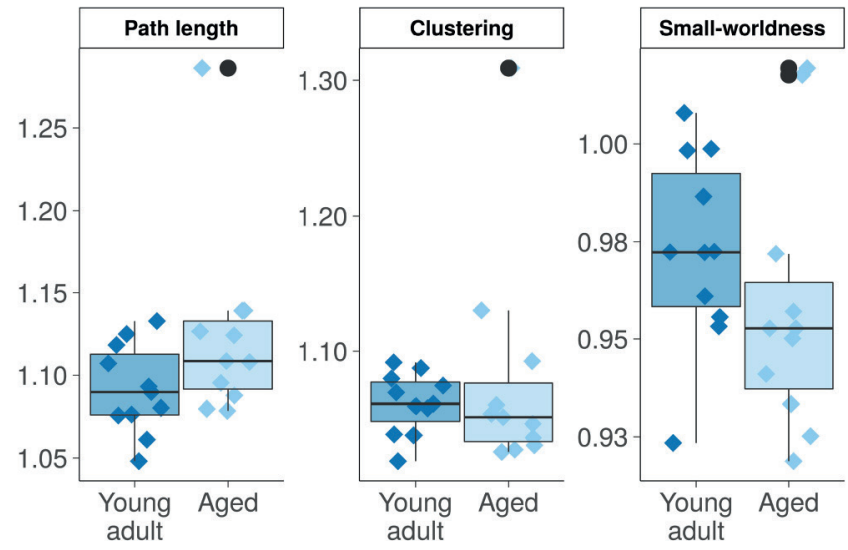

Figure 2. Global network parameters for whole-brain structural and resting-state functional networks in young adult and aged rats. Path length, clustering coefficient and small-worldness in (A) diffusion MRI-based whole-brain structural networks and (B) resting-state fMRI-based whole-brain functional networks in young adult (dark blue) and aged rats (light blue). Individual values are shown as diamonds with horizontal jitter for visualization purposes. Boxplots show median and inter-quartile range (IQR), whiskers representing 1.5 times the IQR, and dots representing outliers. Outliers are defined as values exceeding 1.5 times the IQR above the upper and below the lower quartile. Clustering $=$ Clustering coefficient . 


\section{A) Structural network}

Young adult

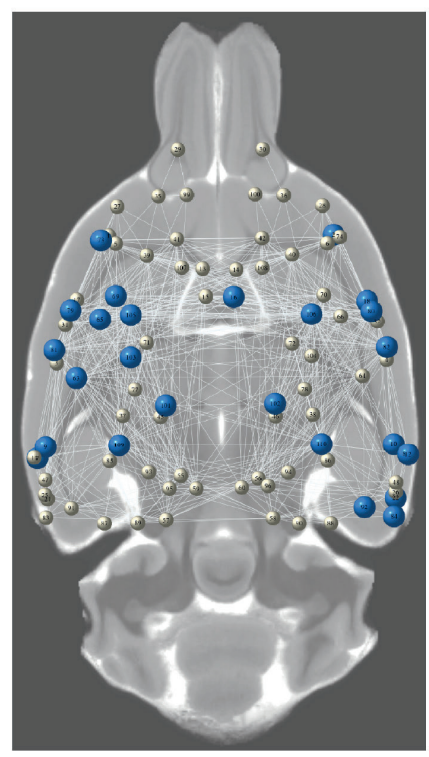

$\begin{array}{ll}\text { Left } & \text { Right } \\ \text { Au1 } & \\ \text { AuD } & \text { AuD } \\ \text { Cpu } & \text { Cpu } \\ \text { GP } & \\ \text { Hipp } & \text { Hipp } \\ \text { S1BF } & \\ \text { S1DZ } & \\ \text { S1FL } & \\ \text { S1J } & \\ \text { S1ULp } \\ \text { S1ULp } \\ \text { S2 } & \text { S2 } \\ \text { T } & \text { T } \\ & \text { AID } \\ & \text { AuV } \\ & \text { Cg2 } \\ & \text { DI } \\ & \text { DLEnt } \\ & \text { TeA } \\ & \text { V2L }\end{array}$

Aged

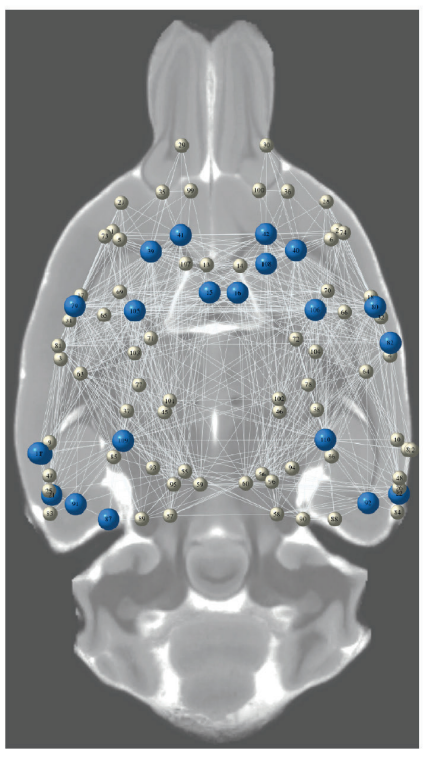

Left Right

Au1

AuV

Cg2 Cg2

Cpu Cpu

DLEnt DLent

Hipp Hipp

M1 M1

M2 M2

S1ULP

S1ULP

V1B

V2L V2L

Acc

S2

\section{B) Functional network}

Young adult

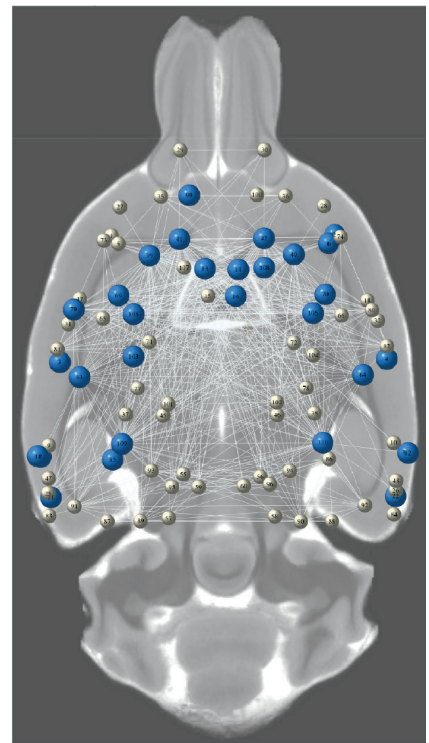

\begin{tabular}{|c|c|}
\hline Left & Right \\
\hline AIP & AIP \\
\hline AuV & AuV \\
\hline $\begin{array}{l}\mathrm{Au} 1 \\
\mathrm{Ca} 1\end{array}$ & \\
\hline & $\begin{array}{l}\mathrm{Cg} 1 \\
\mathrm{Cpu}\end{array}$ \\
\hline $\begin{array}{l}\text { DLEnt } \\
\text { GP }\end{array}$ & DLEnt \\
\hline Hipp & Hipp \\
\hline M1 & M1 \\
\hline M2 & M2 \\
\hline S1BF & S1BF \\
\hline S1FL & S1FL \\
\hline $\begin{array}{l}\text { S1ULp } \\
\text { V1 }\end{array}$ & \\
\hline & \\
\hline & $\begin{array}{l}\text { Acc } \\
\text { Cg2 }\end{array}$ \\
\hline & AID \\
\hline & AIV \\
\hline
\end{tabular}

\section{Aged}

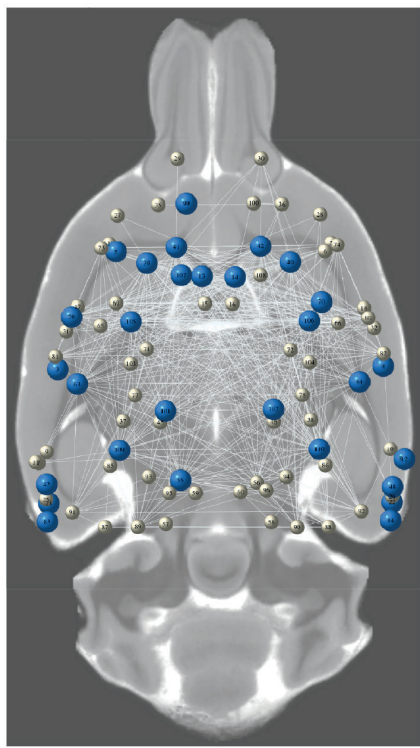

Left Right

Acc

AIP AIP

AIV

AuV

Cg1 Cg1

Cpu Cpu

DLEnt DLEnt

M1 M1

M2 M2

Prh Prh

RSD

S1BF S1BF

S1ULP

Hipp Hipp

$\begin{array}{ll}\mathrm{T} & \mathrm{T}\end{array}$

TeA TeA

VO

S1FL

Figure 3: Hub regions in structural and functional whole-brain networks in young adult and aged rats. Hub regions in (A) structural networks and (B) resting-state functional networks, overlaid on an axial structural MR image of a rat brain slice, are displayed in the 3D network as large blue nodes, whereas the other (non-hub) regions are presented as small white nodes. Networks are shown for young adult (left) and aged rats (right). Hub regions are listed right from the maps. 


\section{Network hub nodes shift between young adulthood and old age.}

Hub nodes, i.e. the nodes with the highest degree of connectivity within the network, characterized by a low clustering coefficient and short path length, and a high strength and betweenness centrality, were found throughout the rat brain. The distribution of hub nodes in the structural brain network is shown in Figure $\mathbf{3 A}$ for young adult and aged rats. Consistent hub nodes in young adult and aged rats were, for example, the left and right caudate putamen and hippocampi. From young adulthood to old age, several regions (e.g. in the left somatosensory cortex) appeared to lose their hub status, whereas other regions (e.g. the left and right primary and secondary motor cortices) acquired a hub status. In aged rats, hubs tended to be more symmetrically distributed in bilateral homotopic areas (e.g., the left and right primary and secondary motor cortices, caudate putamen, and dorsolateral entorhinal cortices), and were located more medially as compared to young adult rats. The overlap in hub nodes in the young adult and aged structural network, expressed by the Dice coefficient was $0.29(95 \% \mathrm{Cl}=0.14-0.44)$. The Dice coefficient (reflecting the overlap between hub regions in the left and right hemisphere) was $0.61(95 \% \mathrm{Cl}=0.31-0.85)$ for aged rats, compared to $0.32(95 \% \mathrm{Cl}=0.11-0.53)$ for young adult rats ( $\Delta$ Dice $=0.30,95 \%$ $\mathrm{Cl}=-0.03-0.62, \mathrm{p}=0.13$ ).

The distribution of hub nodes in the resting-state functional network (Figure 3B) was different from that in the structural network. Nevertheless, consistent with the findings in the structural network, the left and right caudate putamen and hippocampi were also hub nodes in the functional networks of young adult and aged rats. There was a relatively higher density of functional network hub nodes in posterior and temporal regions (such as the temporal association area and perirhinal cortex) in aged rats as compared to young adult rats. The overlap in hub nodes in the young adult and aged functional network never reached 1 (mean Dice: $0.53 ; 95 \% \mathrm{Cl}=0.37-0.68$ ). Regions that lost their hub status in the resting-state functional network in aged rats included the right insular cortex, while other regions, such as the bilateral thalamus, acquired hub status. In line with the structural network hubs, functional network hub nodes in old rats tended to be more symmetrically distributed in homotopic areas in the left and right hemispheres. The Dice coefficient (reflecting the overlap between hub regions in the left and right hemisphere) was 0.61 (95\% $\mathrm{Cl}=0.39-0.83)$ for old rats, compared to $0.53(95 \% \mathrm{Cl}=0.32-0.74)$ for young adult rats ( $\Delta$ Dice $=0.08,95 \% \mathrm{Cl}=-0.14-0.32, \mathrm{p}=0.44)$. 


\section{A) Global structural network}

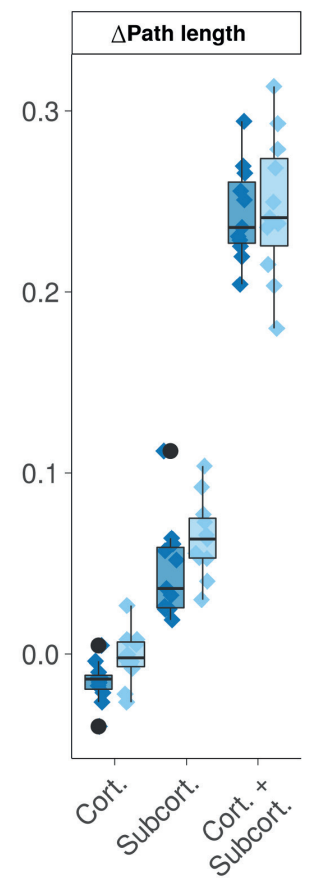

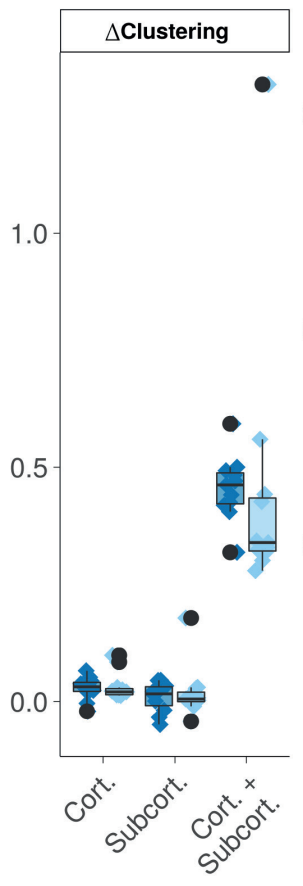

B) Global functional network

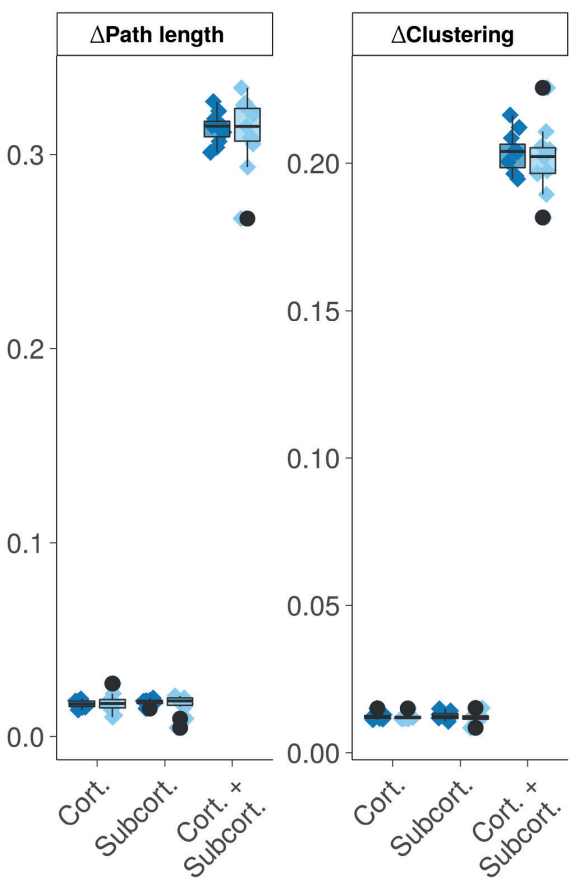

Figure 4. Effects of different stroke lesion simulations on global network measures in young adult and old rats. Changes in path length, clustering and small-worldness after stroke lesion simulation, calculated as metric delta values between the healthy control network and the network after stroke simulation, for (A) diffusionbased structural networks and (B) resting-state fMRIbased functional networks. Delta-scores were determined for different stroke lesion simulations, i.e. cort: cortical (photothrombosis),

subcort: subcortical (short transient MCAO) and cort + subcort: cortical plus subcortical (long transient or permanent MCAO), for young adult rats (dark blue) and aged rats (light blue). Individual values are given as diamonds with horizontal jitter for visualization purposes. Boxplots show median and inter-quartile range (IQR), whiskers representing 1.5 times the $I Q R$, and dots representing outliers. Outliers are defined as values exceeding 1.5 times the IQR above the upper and below the lower

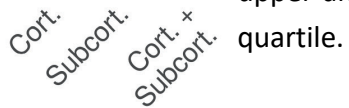




\section{Lesion simulations}

To determine the effect of stroke lesions on network parameters in young adult and old rats, we simulated the extent of network damage for different frequently applied stroke models (cortical photothrombosis, transient MCAO with only subcortical damage, and transient/permanent MCAO with cortical and subcortical damage). We calculated a deltascore for each network parameter as the change in the network parameter after lesion simulation as compared to the parameter for the healthy network.

\section{Unilateral stroke lesion simulations affect global structural and functional network features at a whole-brain level.}

The effect of stroke lesion simulations on global structural network characteristics are shown in Figure 4A. Subtle, but statistically significant changes were measured after cortical (i.e. increased clustering $(p<0.01)$ ) and subcortical stroke simulations (i.e. increased path length and decreased small-worldness $(p<0.01)$ ), which were comparable between young adult and old aged rats. Subcortical plus cortical damage resulted in considerable increases in path length and clustering in both age groups $(p<0.01)$.

For whole-brain functional networks, stroke lesion simulations resulted in significant changes for all measured global network characteristics, i.e. increased path length, higher clustering and lower small-worldness ( $p<0.01$; Figure 4B). These changes were relatively small for the cortical and subcortical stroke simulations, and substantial for the large stroke lesions involving cortical plus subcortical regions. There were no significant differences in these global functional network changes between young adult and aged animals.

Additional analyses of lesion simulations in the right hippocampus (hub region) or right medial parietal association cortex (non-hub region) revealed subtle but significant effects on structural and functional networks, which were highly comparable with the effects of cortical stroke and sub-cortical stroke (Figure S6).

\section{Hub regions shift after stroke simulations}

In addition to changes in global network features, stroke lesion simulations resulted in shifts of hub nodes in young adult and aged rats' structural and functional networks (Figures $\mathbf{5}$ and 6). These shifts were most apparent after simulation of a large cortical plus subcortical lesion. In structural networks, some regions acquired a hub status, whereas other regions lost their hub status after lesion simulations (Figure 5). In functional networks, regions mainly acquired a hub status after lesion simulations (Figure 6). The number of regions with shifted hub status was higher in structural networks than in functional networks, indicated by a lower Dice coefficient for the overlap in hub regions before and after stroke simulation (Table 3). 
A) Small cortical lesion
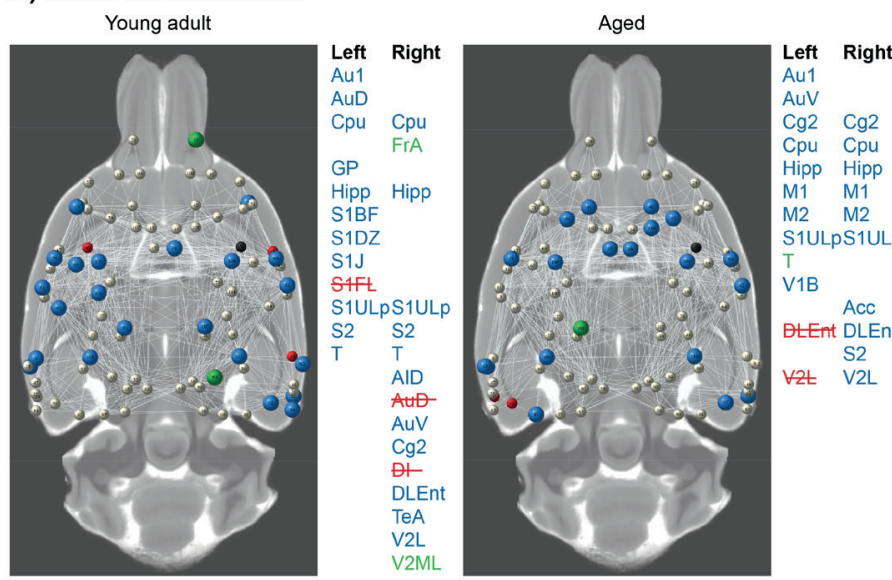

\section{B) Subcortical lesion}
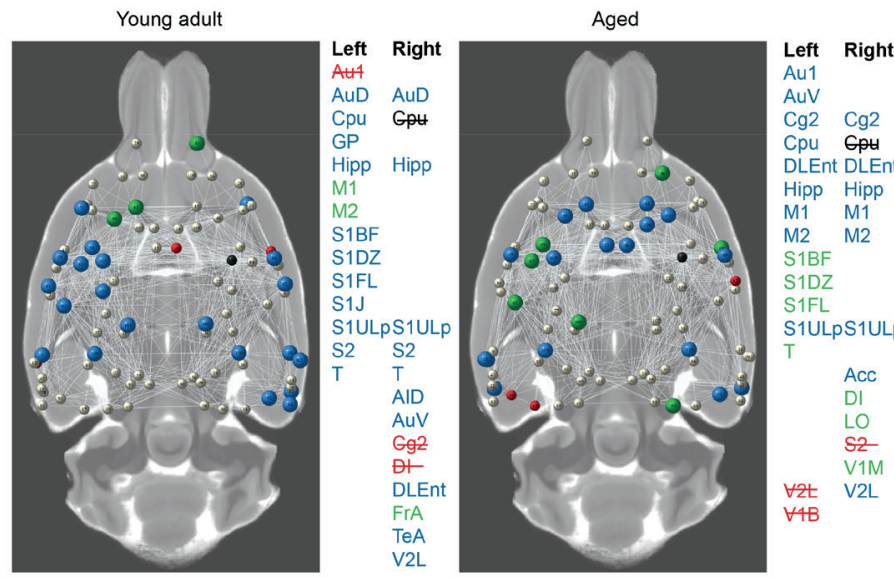

C) Large cortical and subcortical lesion

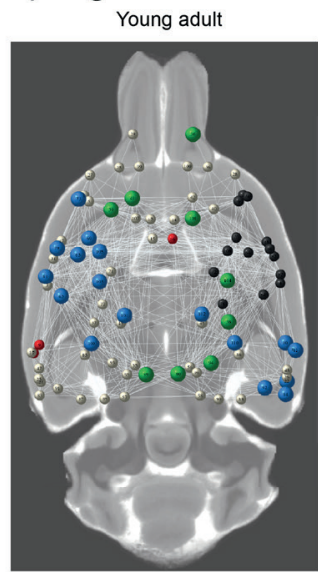

Figure 5. Hub shifts in structural whole-brain networks after unilateral stroke lesion simulations in young adult and aged rat brain. Hub shifts are displayed after unilateral (right sided) stroke lesion simulation in (A) a small cortical area, (B) a subcortical area, and (C) a large cortical and subcortical area. Hub nodes, overlaid on an axial structural MR image of a rat brain slice, are displayed in the 3D network as large nodes, whereas other (non-hub) regions are represented as small (white) nodes. Regions with maintained hub station after stroke lesion simulation are shown as large blue nodes, whereas regions that acquired a hub status are presented as large green nodes. Regions that lost hub status are presented as small red nodes. Lesioned nodes are presented in darkgrey color. Networks are shown for young adult (left) and aged rats (right). Hub regions are listed right from the maps. Regions with acquired hub status are shown in green, and regions with lost hub status are crossed-out in red or black (lesioned nodes). 
A) Small cortical lesion
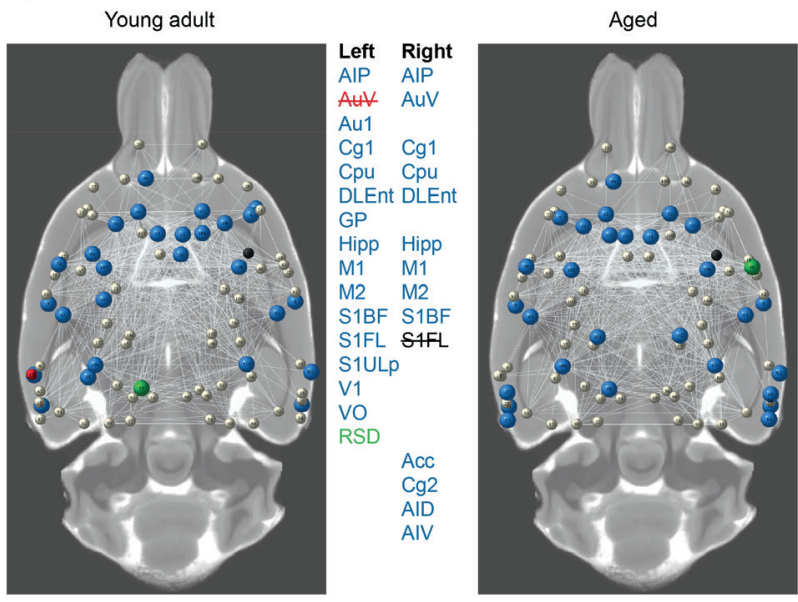

\section{B) Subcortical lesion}
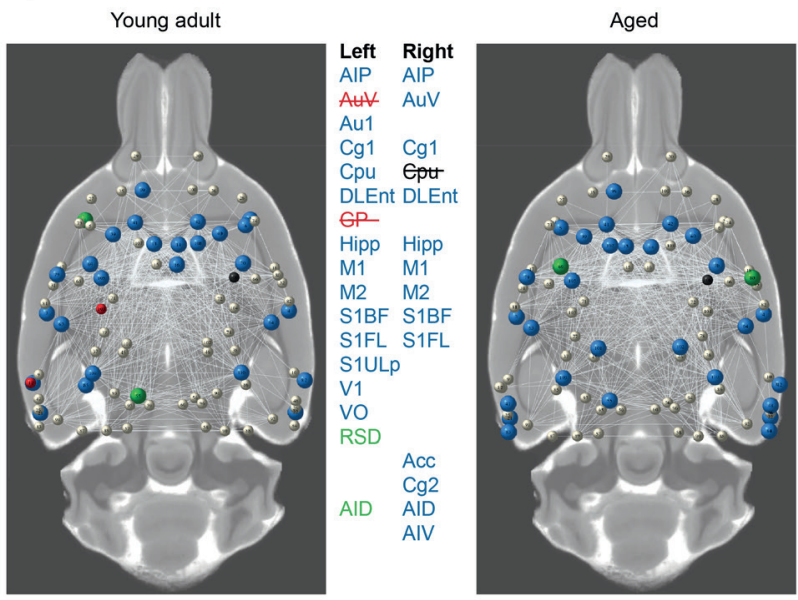

\section{C) Large cortical and subcortical lesion}

Young adult

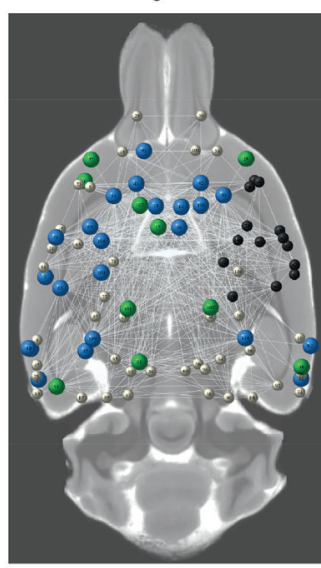

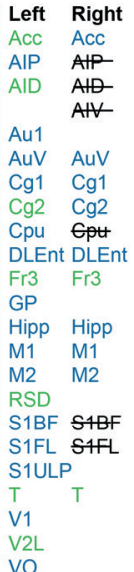

Aged

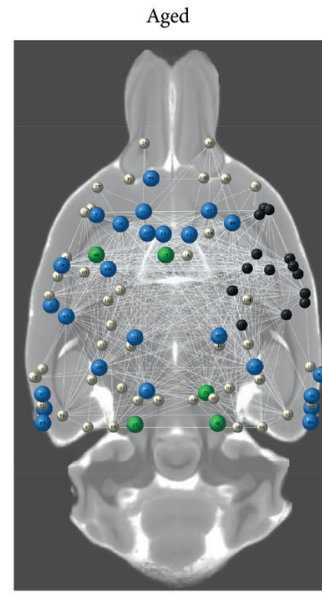

Left Right

ACC

AIP AIP

AuV

Cg1 Cg1

Cpu Epu

DLEnt DLEnt

M1 M1

M2 M2

Prh Prh

RSD

S1BF S1BF S1ULPS1ULP S1FL S1FL Hipp Hipp $\begin{array}{ll}T & T \\ \text { TeA } & \text { TeA }\end{array}$ VO

Left Right

ACC

AIP AIP

AuV

Cg1 Cg1

$\mathrm{Cg} 2$

Cpu Epu

DLEnt DLEn

M1 M1

M2 M2

Prh Prh

RSD RSD

RSGa RSGa

S1BF S1BF

S1ULp

S1FL SHFL

Hipp Hipp

$\begin{array}{ll}T & T \\ T & \end{array}$

VO
Figure 6. Hub shifts in

functional whole-

brain networks after

unilateral stroke

lesion simulations in young adult and aged

rat brain. Hub shifts are displayed after unilateral (right-sided) stroke lesion simulation in (A) a small cortical area, (B) a subcortical area and (C) a large cortical and subcortical area. Hub nodes, overlaid on an axial structural MR image of a rat brain slice, are displayed in the 3D network as large nodes, whereas other (non-hub) regions are represented as small (white) nodes. Regions with maintained hub status after stroke lesion simulation are shown as large blue nodes, whereas regions that acquired a hub status are presented as large green nodes. Regions that lost hub status are presented as small red nodes. Lesioned nodes are presented in darkgrey color. Networks are shown for young adult (left) and aged rats (right). Hub regions are listed right from the maps. Regions with acquired hub status are shown in green, and regions with lost hub status are crossed-out in red or black (lesioned nodes). 
As can be observed in Figures $\mathbf{5}$ and $\mathbf{6}$, there were several local differences between young adult and old rats in the pattern of shifts in hub regions in structural and functional networks after stroke simulations. For example, in aged rats we found that the left, contralesional forelimb region of the primary somatosensory cortex acquired a functional network hub status after a unilateral lesion in subcortical or cortical plus subcortical tissue, which was not observed in young adult rats.

\begin{tabular}{lllll}
\hline & \multicolumn{2}{c}{ Structural network } & & \multicolumn{2}{l}{ Functional network } \\
\cline { 2 - 5 } & Young adult & Aged & Young adult & Aged \\
\hline Cortical stroke & 0.78 & 0.86 & 0.90 & 0.93 \\
& $(0.61-0.93)$ & $(0.70-1.00)$ & $(0.78-1.00)$ & $(0.83-1.00)$ \\
\hline Subcortical stroke & 0.71 & 0.61 & 0.84 & 0.90 \\
& $(0.54-0.88)$ & $(0.42-0.79)$ & $(0.70-0.96)$ & $(0.79-1.00)$ \\
\hline Cortical plus & 0.47 & 0.44 & 0.55 & 0.71 \\
subcortical stroke & $(0.30-0.64)$ & $(0.26-0.62)$ & $(0.39-0.71)$ & $(0.55-0.85)$ \\
\hline
\end{tabular}

Table 3. Dice coefficients $(95 \% \mathrm{Cl})$ for the overlap in hub regions before and after stroke simulations, in structural and functional brain networks of young adult and old rats.

\section{DISCUSSION}

In this study, we investigated whether the effects of simulated lesions - representing topographical profiles of different rodent stroke models - on structural and functional network organization in rat brain differed between young adulthood and old age. Overall, global network features were largely comparable, however, local differences in structural and functional networks were observed between young adult and aged rats, particularly expressed by shifts in hub regions in the brain. Unilateral stroke simulations induced global changes in whole-brain structural and functional network organization, which was most significant for the large stroke simulation, involving cortical and subcortical lesioning. Global network changes after stroke simulations were comparable between young adult and aged rats. On the other hand, lesion-induced regional changes in hub status, which were more pronounced in the structural than in the functional brain network, differed between young adult and aged rats.

\section{Whole-brain structural and functional networks - effects of aging}

The measured similarity of global network characteristics between healthy young adult and aged rats, as measured from the path length, clustering and small-worldness in the structural and functional networks, is in line with human data. Small-world topology has been demonstrated in structural brain networks of young and aged adult humans (Gong et al., 2009; Zhu et al., 2012), and no aging effect was found for global network efficiency (Gong et al., 2009). However, other studies reported lower global efficiency in younger individuals (Wu et al., 2012; Zhu et al., 2012). This discrepancy may be explained by the use of different age categories across studies. It has been demonstrated that global efficiency 
follows an inverted u-curve pattern between young and old age (Wu et al., 2012). Because the age groups in our study were at the extremities of this curve, we may have missed possible changes in global network characteristics that develop between the two timepoints.

In functional networks, human resting-state fMRI studies have shown an increased minimum path length and clustering coefficient in older adults (Sala-Llonch et al., 2014). In addition, task-fMRI studies during memory encoding and recognition in healthy individuals demonstrated a similar increase in path length with aging (Wang et al., 2010). Although we did not find statistically significant aging effects on global networks, we did see a trend towards a higher path length and lower small-worldness in the functional network of old rats, resembling the described effects of aging in humans.

While global network features were largely the same, we identified local differences in structural and functional brain networks between young adult and aged rats. Node strength increased for many regions, such as the bilateral primary and secondary motor cortices and caudate putamen, within the whole-brain structural network, which seems in contrast to reductions in structural connectivity during aging as observed in humans (Gong et al., 2009). Loss of structural connectivity may be reflective of white matter degeneration in aging brains (Meier-Ruge et al., 1992; Salat et al., 2005). However, white matter degeneration may not be similarly prominent in aging rats. In fact, diffusion tensor imaging studies have revealed ongoing brain maturation in rats during adulthood (Mengler et al., 2014) with increasing fractional anisotropy in rodent white matter structures up to old age (Blockx et al., 2011). Furthermore, the mean diameter and volume density of myelinated fibers in the cortex are higher while the length density is lower, which may be explained by specific loss of thinner myelinated fibers, in aged as compared to younger adult rats (Zhang et al., 2009). These white matter differences can affect the diffusion-based tractography pattern and explain changes in node strengths that we observed between young adults and aged rats.

In addition to node strength differences in structural networks, we found shifts in hub regions from young adulthood to old age in structural and functional networks. Increased homotopical symmetry of hub regions in aged rats may be reflective of altered lateralization, which has also been reported for aged humans (Agcaoglu et al., 2015). Also, increasing homotopic connectivity with age, particularly between sensorimotor regions, has been found in a human resting-state fMRI study (Zuo et al., 2010). Moreover, similar to studies in human subjects, we observed a posterior shift in hub regions in the functional network, exemplified by hub status for the bilateral temporal association areas and perirhinal cortex in aged rats, although in humans this has been explained by lost hub status of frontal brain regions due to decreasing local network efficiency (Achard and Bullmore, 2007; Meunier et al., 2009). 


\section{Whole-brain structural and functional networks - effects of stroke lesion simulations}

Computational lesion studies, as recently reviewed by Aerts et al. (Aerts et al., 2016), may substitute, complement or guide empirical studies in humans or animal models. Lesion simulation studies can be applied to already existing data, and provide a simple, cheap and non-invasive alternative to complicated longitudinal in vivo lesion studies, thereby contributing to replacement, reduction and refinement of animal research (Balls et al., 1995).

In the current study we simulated unilateral stroke lesions, based on lesion topographies of three popular rat stroke models. Additionally, we simulated focal lesions in a single hub (i.e. right hippocampus) or non-hub region (i.e. right medial parietal association cortex) region. Significant changes in global network features were measured in whole-brain structural and functional networks. Results from our in silico lesion simulation study are largely in agreement with our previous in vivo resting-state fMRI study, in which we measured changes in the bilateral sensorimotor network after transient MCAO in young adult rats (van Meer et al., 2012). In both studies we found a longer path length and higher clustering coefficient in functional networks in response to stroke lesions in subcortical, or cortical plus subcortical tissue. The effects were considerably smaller when lesions were confined to only subcortical or cortical tissue, regardless whether this was a hub (i.e. hippocampus or $\mathrm{CPu}$ ) or a non-hub region (i.e. medial parietal association cortex), which reflects robustness of the functional and structural networks against relatively small focal injury. Apparently, this was not affected by age, because the results were similar in young adult and aged rats.

Our stroke lesion simulations also resulted in clear shifts of hub regions in the structural and functional networks. Network hub regions are believed to be key players in the outcome of brain disorders (Crossley et al., 2014; Stam, 2014). Hub damage may have severe consequences for network function, while hub shifts may contribute to network remodeling. In our study, the number of hub shifts was higher in structural than in functional networks, which we observed in young adult as well as old rats. We speculate that this relates to the dependency of functional connectivity on direct as well as indirect structural connections (Adachi et al., 2012; Honey et al., 2009), which may facilitate network resilience. Since we only removed nodes and their direct connections in our lesion simulations, diffusion-based structural networks, which in essence only contain direct connections, would be more severely affected.

The lesion-induced hub shifts were different between young adult and aged rats. In aged rats contralesional somatosensory areas acquired hub status after subcortical or cortical plus subcortical stroke lesion simulations, which was not observed in young adult rats. This may relate to enhanced neural activity or functional connectivity in the contralesional hemisphere, which has been measured in stroke patients and animal models (Grefkes and 
Fink, 2014; van Meer et al., 2010a, 2010b). Why increased involvement of contralesional sensorimotor areas after unilateral stroke appeared more specifically in aged brain in our simulation study, and whether it may lead to worsening of functional outcome or contribute to functional recovery, remains to be elucidated. The local differences in structural and functional brain networks, including hub regions, between healthy young adult and aged rats, may have contributed to the different effects of lesion simulations between the two age groups. This underlines the critical role of age in the outcome of preclinical stroke studies in animal models, which often only involve young adult rodents, thereby limiting bench-to-bedside translation (Dirnagl, 2016).

It is important to realize that our computational simulation study only assessed direct effects of lesions on network status by elimination of nodes. Dynamic network responses, such as subsequent degeneration or regeneration, which can strongly depend on age (Betzel et al., 2014; Burke and Barnes, 2006; Damoiseaux, 2017; Niccoli and Partridge, 2012; Wang et al., 2015), were not accounted for. Another limitation is the use of anesthesia during resting-state $\mathrm{fMRI}$, which is known to affect functional connectivity measurements (Paasonen et al., 2018). However, it might be argued that functional connectivity in anesthetized animals better reflects 'resting state' (i.e. baseline) connectivity than functional connectivity under awake conditions, which may be significantly affected by stress and motion. We used the same anesthesia protocol for all developmental stages, which enabled us to compare age effects under the same conditions. Nevertheless, future studies should look into (differences in) effects of anaesthesia on network parameters across the lifespan and after stroke.

A limitation of diffusion-based tractography for structural connectivity measurement, is that white matter tracts are reconstructed from the underlying diffusion profiles, with limited power to resolve complex fiber configurations (e.g. crossing, bending and fanning fibers). This can result in considerable amounts of false positive and false negative connections (Calabrese et al., 2015; Jeurissen et al., 2017; Sinke et al., 2018; Thomas et al., 2014). Nevertheless, diffusion-based tractography is currently the only method to map whole-brain structural connections in vivo at the macro-scale. For our study we used a stateof-the-art approach, combining CSD-based tractography - which partially accounts for crossing fibers - with whole-brain filtering of tracts, which has been shown to yield biologically accurate connectomes (Smith et al., 2013, 2015a).

Future research involving longitudinal in vivo imaging studies and improved structural and functional connectivity reconstruction algorithms combined with advanced network analysis strategies may provide further insights in the causes and consequences of agerelated differences in susceptibility and resilience to stroke injury. In the end, knowledge of the structural and functional network status of a stroke patient's brain, could guide 
selection of appropriate recovery-enhancing treatment strategies targeted at optimal engagement of a patient's intact neural circuitry.

In conclusion, our study showed that global structural and functional network features are largely similar in young adult and old aged rat brains, and respond comparably to different types of stroke simulations. On the other hand, local network differences, particularly reflected by shifts in hub nodes, were identified between the brains of young adult and aged rats, before and after simulated stroke. These age-dependent neural network changes may play a critical role in the vulnerability and resilience to (strokeinduced) brain injury. 


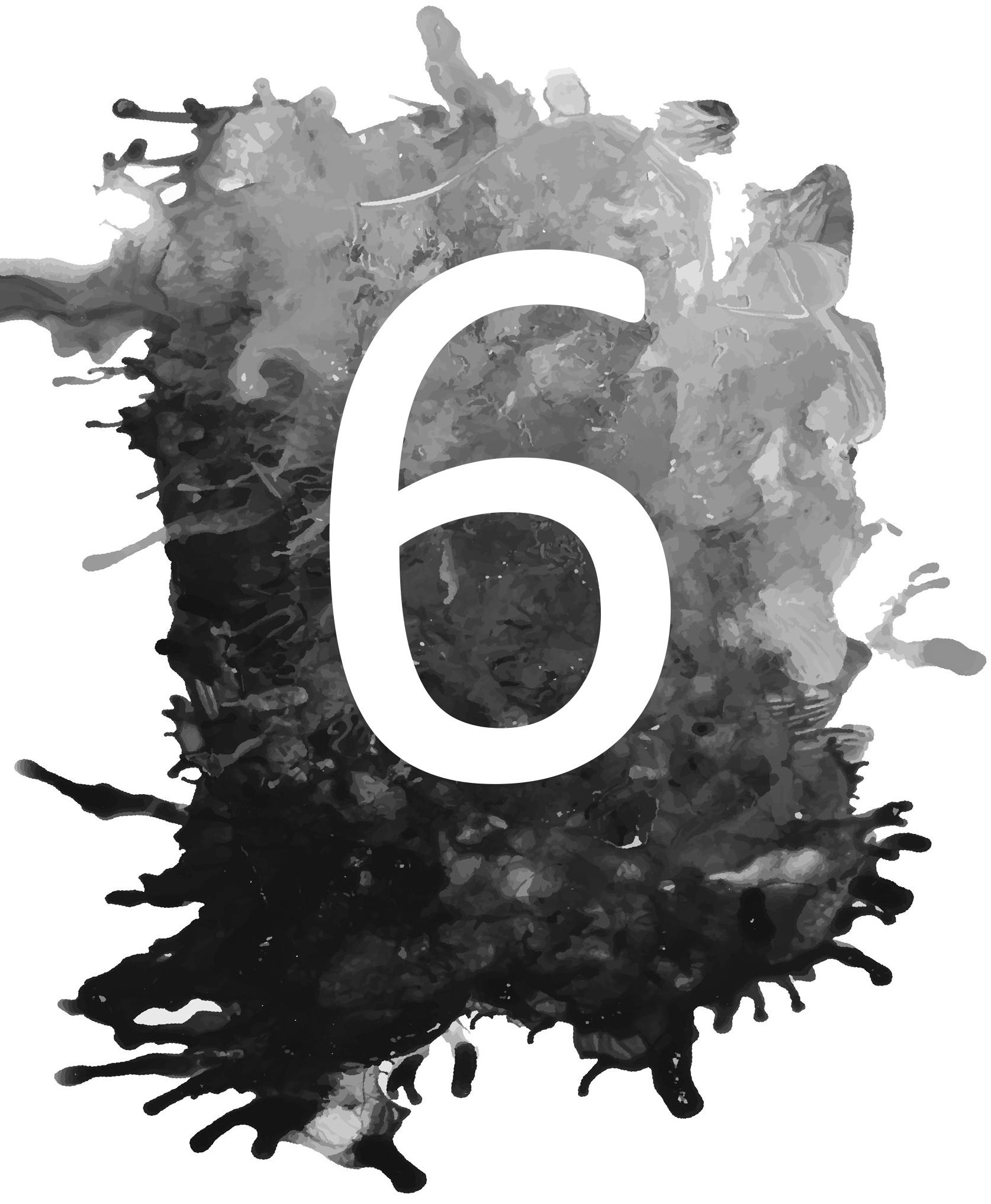




\section{Chapter 6}

\section{Remote Corticospinal Tract Degeneration after Cortical Stroke in Rats May Not Preclude Spontaneous Sensorimotor Recovery}

M.R.T. Sinke, G.A.F. van Tilborg, A.E. Meerwaldt, C.L. van Heijningen, A. van der Toorn, F. Rakib, M.H.M. Ali, K. Al-Saad, W.M. Otte \& R.M. Dijkhuizen 


\begin{abstract}
Recovery of motor function after stroke appears to be related to the integrity of axonal connections in the corticospinal tract (CST) and corpus callosum, which may both be affected after cortical stroke. In the present study we aimed to elucidate the relationship of changes in measures of the CST and transcallosal tract integrity, with the interhemispheric functional connectivity and sensorimotor performance after experimental cortical stroke. We conducted serial in vivo diffusion MRI, resting-state functional MRI and behavior testing in twenty-five male Sprague Dawley rats recovering from unilateral photothrombotic stroke in the sensorimotor cortex. Twenty-three healthy rats served as controls. A reduction in the number of reconstructed fibers, a lower fractional anisotropy (FA) and higher radial diffusivity in the ipsilesional but intact CST, reflected remote white matter degeneration. In contrast, transcallosal tract integrity remained preserved. Functional connectivity between the ipsi- and contralesional sensorimotor cortices recovered after 28 weeks following initial loss at week one and eight post-stroke. This recovery paralleled the time-course of sensorimotor performance. Our study shows that post-stroke motor recovery is possible despite degeneration in the CST, and may rely on intact neuronal communication between hemispheres.
\end{abstract}




\section{INTRODUCTION}

Stroke is one of the most prevalent neurological disorders worldwide and one of the main causes of adult disability, affecting almost 17 million people throughout the world (Feigin et al., 2014; Sousa et al., 2009). The majority of stroke survivors experiences long-term sensorimotor and cognitive dysfunction. However, in the weeks and months following a stroke, many patients show partial recovery of sensorimotor (or cognitive) functions. In many cases, functional recovery obeys the recently postulated 'proportional recovery rule', which states that patients should reach about $70 \%$ of their maximum potential recovery at three months post-stroke (Krakauer and Marshall, 2015). Cross-species validity of the proportional recovery rule has recently been demonstrated in a large study with rats recovering from ischemic stroke (Jeffers et al., 2018). However, the proportional recovery rule does not apply invariably and many patients experience limited or no functional recovery over time (Krakauer and Marshall, 2015). Furthermore, there has been debate in recent papers about the suitability of the proportional recovery rule from a statistical and methodological perspective (Hope et al., 2019; Kundert et al., 2019). This strongly emphasizes the need for improved understanding of the underlying mechanisms of (spontaneous) functional recovery after stroke, and the identification of biomarkers that can accurately predict functional outcome.

Post-stroke functional recovery has been related to reorganization and restoration of surviving ipsi- and contralesional networks in the brain (Cramer, 2008; Grefkes and Fink, 2011; Jiang et al., 2013; Jones, 2017; Murphy and Corbett, 2009). A critical factor for functionally effective neuronal reorganization within the motor system appears to be the integrity of axonal connections in the corticospinal tract (CST) and corpus callosum. However, after stroke these pathways may be structurally or functionally disrupted, thereby directly affecting motor function along with potential for motor recovery in stroke survivors (Carter et al., 2012a; Crofts et al., 2011; Grefkes and Fink, 2011; van Meer et al., 2010b; Rehme and Grefkes, 2013).

Structural and functional connectivity can be mapped at a whole-brain level with in vivo magnetic resonance imaging (MRI) techniques, such as diffusion MRI and functional MRI. Diffusion MRI indirectly probes the arrangement of axonal projections from the random diffusion of water molecules in and around axonal fiber bundles (Basser et al., 2000; Jbabdi and Johansen-Berg, 2011). Diffusion MRI studies in stroke patients have revealed reduced fractional anisotropy (FA) and increased diffusivity values in the ipsilesional CST, indicative of axonal degeneration. Higher FA and lower diffusivity values are associated with improved motor functions at chronic stages (Lindberg et al., 2007; Lindenberg et al., 2010, 2013; Schaechter et al., 2010). Also, measures of the intactness of transcallosal tracts have been shown to be predictive of good motor outcome (Lindenberg et al., 2013; van Meer et al., 2012). 
Functional connectivity within brain networks may be assessed with resting-state functional MRI (rs-fMRI), which measures inter-regional temporal correlation of lowfrequency spontaneous fluctuations in blood oxygenation level-dependent (BOLD) signals, reflective of neuronal synchronization. Clinical and pre-clinical studies have reported poststroke loss of functional connectivity, for example between the left and right motor cortices, which may recover over time, in parallel with recovery of motor function (Carter et al., 2010; Grefkes and Fink, 2011, 2014; van Meer et al., 2010a, 2010b, 2012). These findings also point toward the importance of transcallosal tracts - and their integrity - for functional recovery.

Despite increasing insights into the importance of CST and transcallosal tracts for motor recovery after stroke, the relationships between stroke injury, motor function, and structural and functional connectivities within the remaining post-stroke motor network remain unknown. In the present study we aimed to elucidate the relationship of longitudinal changes in measures of CST and transcallosal tract integrity versus cortical injury, functional connectivity and motor performance after experimental cortical stroke. To that aim we acquired serial in vivo diffusion MRI and rs-fMRI data and behavioural scores from rats recovering from unilateral focal stroke in the motor cortex.

\section{METHODS}

\section{Animals}

Ethical approval was given by the Animal Experiments Committee of the University Medical Center Utrecht and the Utrecht University, and experiments were performed in accordance with the guidelines of the European Communities Council Directive.

The present study was performed with 48 adult male Sprague-Dawley rats (Charles River, Germany). Animals were allowed to acclimatize at least 7 days after delivery. Animals were housed under standard conditions with a light/dark cycle of 12/12 hours, with light on time at 7:00 a.m, and ad libitum access to food and water. A power calculation was conducted with Gpower 3.1.5. A sample size $(n=7)$ per time point was computed based on a t-test for the difference between independent means, with $\alpha=0.05$, power $=80 \%$ and an effect size of 1.5. The effect size was based on FA values in the anterior internal capsule of rats 7 days post-stroke versus control rats without stroke from a previous study (van Meer et al., 2012). Hence, a total of 25 animals were subjected to experimental stroke surgery. Three animals died as a consequence of stroke surgery before MRI acquisition. MRI was performed in control animals and animals with stroke at 1 week ( $n=7$ control, $n=8$ stroke), 8 weeks ( $n=8$ control, $n=7$ stroke) or 28 weeks ( $n=8$ control, $n=7$ stroke). In addition, each rat underwent behavioral testing (during light-on time) at multiple time points, up to the day of MRI. After MRI, rats were euthanized with an overdose of isoflurane, 
transcardially perfused with $4 \%$ paraformaldehyde, and brains were harvested for immunohistochemistry.

\section{Stroke model}

Photothrombotic stroke was induced in the right sensorimotor cortex of adult male Sprague-Dawley rats $(n=25)$. Surgery was performed under isoflurane anaesthesia supplied in a mixture of $\mathrm{O}_{2}$ /air (1/4) (4\% for induction; $1.5-2.0 \%$ for maintenance). Rats were mechanically ventilated (UNO Micro Ventilator-03, UNO b.v., The Netherlands) through an endotracheal tube (Abbocath ${ }^{\mathrm{TM}}-\mathrm{T}, 14 \mathrm{G} \times 51 \mathrm{~mm}$, ICU Medical, USA). Blood oxygenation and heart rate were continuously monitored (Nonin Medical $8500 \mathrm{~V}$ pulse oximeter) and body temperature was maintained at $37 \pm 0.5{ }^{\circ} \mathrm{C}$. Rats were placed in a stereotaxic frame. The scalp was opened, and lidocaine (Xylocaine $100 \mathrm{mg} / \mathrm{ml}$, AstraZeneca BV, Zoetermeer, The Netherlands) was sprayed on the periosteum, after which the periosteum was removed and the skull was allowed to dry. Next, the illumination area was delineated between +4 to -4 $\mathrm{mm}$ anterior/posterior and 1.5 to $4.5 \mathrm{~mm}$ lateral to bregma, using black tape. Subsequently, an optic fiber (diameter $4.5 \mathrm{~mm}$ ), mounted on a cold light source (Schott KL 1500 LCD, Germany), with a green fluorescent filter (wavelength $515 \mathrm{~nm}$ ), was placed on the skull. The light source was used at a color temperature of 3200K (setting 5), and aperture setting E. Prior to illumination, the photosensitive dye Rose Bengal (Sigma) was infused through the vena saphena $(25 \mathrm{mg} / \mathrm{kg}$ ) at an infusion rate of $5.625 \mathrm{mg} / \mathrm{min}$ in a darkened room. Directly after infusion, illumination was started, which lasted for 20 minutes. Following illumination, the skin on the leg and head was sutured, and the animals were placed on a heating pad in order to recover. Once awake, animals received one single dose $(0.05 \mathrm{mg} / \mathrm{kg})$ of buprenorphine (Temgesic, Schering-Plough).

\section{MRI acquisition}

MRI was performed on a 9.4T horizontal bore Varian MR System (Palo Alto, CA, USA), equipped with a gradient insert with an inner diameter of $12 \mathrm{~cm}$ and a maximum gradient strength of $400 \mathrm{mT} / \mathrm{m}$ at a rise time of $130 \mu \mathrm{s}$. A home-built Helmholtz volume coil $(90 \mathrm{~mm}$ diameter) and an actively decoupled surface coil (25 $\mathrm{mm}$ diameter) were used for excitation and reception, respectively.

For $\mathrm{MRI}$, rats were anesthetized (4\% isoflurane induction; $1.5-2.0 \%$ isoflurane maintenance in $\mathrm{O}_{2}$ /air (1/4)), endotracheally intubated (Abbocath ${ }^{\mathrm{TM}}-\mathrm{T}, 14 \mathrm{G} \times 51 \mathrm{~mm}$, ICU Medical) and mechanically ventilated (TOPO Small Animal Ventilator Kent Scientific). Oxygen saturation, heart rate (Nonin Medical $8600 \mathrm{~V}$ pulse oximeter) and expired $\mathrm{CO}_{2}$ (Microstream handheld capnograph, Oridion) were continuously measured and kept within physiological range. Temperature was maintained at $37.0 \pm 0.5^{\circ} \mathrm{C}$. To minimize motion artifacts, animals' heads were fixed with ear- and toothbars inside a home-built animal 
cradle. Rat heads were carefully positioned underneath the surface coil to obtain full coverage of the cerebrum.

Voxel-based shimming was performed on a rectangular voxel covering the cerebrum and a small fraction of the olfactory bulb and cerebellum, using gradient-echo 3D shimming. Directly following shimming, inspired isoflurane levels were reduced to $1.5 \%$ and the imaging protocol was started.

The imaging protocol consisted of anatomical imaging, rs-fMRI and diffusion MRI. Anatomical images were acquired using a balanced steady-state free precession sequence with an isotropic spatial resolution of $250 \mu \mathrm{m}$ (repetition time $(T R)=5 \mathrm{~ms}$, echo time (TE) = $2.5 \mathrm{~ms}$, flip angle $=20^{\circ}$, field of view $($ FOV $)=40 \times 32 \times 24 \mathrm{~mm}^{3}$, matrix $=160 \times 128 \times 96,3$ averages and pulse angle shifts of $0^{\circ}, 90^{\circ}, 180^{\circ}$ and $270^{\circ}$, total scan time $=12 \mathrm{~min}$ ).

Resting-state fMRI (rs-fMRI) was performed using a single-shot 3D gradient-echo EPI sequence with an isotropic spatial resolution of $600 \mu \mathrm{m}$ (TR $=730.8 \mathrm{~ms}$, TE $=15 \mathrm{~ms}$, flip angle $=13^{\circ}$, bandwidth $=250 \mathrm{kHz}$, FOV $=32.4 \times 32.4 \times 16.8 \mathrm{~mm}^{3}$, matrix $=54 \times 54 \times 28$, transverse orientation, 1 average, 800 images, total scan time $\approx 9.5 \mathrm{~min}$ ).

Directly after rs-fMRI, inspired isoflurane level was increased to $2 \%$, and maintained until the end of the MRI protocol. Diffusion MRI was executed with a 4-shot 2D spin-echo EPI sequence with diffusion-weighting (TR $=1.7 \mathrm{~s}, \mathrm{TE}=34 \mathrm{~ms}$, bandwidth $=250 \mathrm{kHz}$, FOV = $32 \times 32 \mathrm{~mm}^{2}$, matrix $=64 \times 128,25$ transverse slices of $0.5 \mathrm{~mm}$ thickness, 4 averages, 30 gradient directions, diffusion gradient strength $=19 \mathrm{G} / \mathrm{cm}, \Delta=15.5 \mu \mathrm{s}, \delta=6.5 \mu \mathrm{s}, \mathrm{b}=1471$ $\mathrm{s} / \mathrm{mm}^{2}$, total scan time $=14.5 \mathrm{~min}$ ). For each acquisition, two equivalent $\mathrm{b}_{0}$ images without diffusion-weighting were also obtained.

\section{MRI data processing}

Acquired MR images were post-processed with FSL 5.0 software (Jenkinson et al., 2012).

\section{Anatomical imaging - Lesion segmentation}

The brain voxels in the anatomical images were extracted (Smith, 2002). Images were corrected for signal inhomogeneities. The eight brain scans acquired at one week poststroke were registered to the brain scan of one reference rat from the one week stroke group, with affine transformation (FLIRT) (Jenkinson and Smith, 2001; Jenkinson et al., 2002) followed by non-linear transformation (FNIRT) (Andersson et al., 2007). Brain lesions were manually segmented in the aligned anatomical images using FSLview. Subsequently, a lesion incidence map was calculated.

\section{Diffusion tensor imaging and tractography}

Brain voxels in the diffusion scans were extracted, based on the $b_{0}$ images (Smith, 2002). Next, FSL's DTIFIT was used to calculate FA, mean diffusivity (MD), axial diffusivity (AD) and 
radial diffusivity $(\mathrm{RD})$ from the masked diffusion data. Images from two control animals at one week were excluded from further analyses because of poor image quality. Deterministic diffusion tensor-based whole-brain tractography was performed in MRtrix3 (Tournier et al., 2012), generating 250.000 streamlines with a step size of $25 \mu \mathrm{m}$, an FA threshold $\geq 0.3$ and an angle threshold of $30^{\circ}$ (Figure S1).

Prior to selection of the corticospinal and transcallosal tracts, image registration was performed according to the following procedure. After signal inhomogeneity correction, the $b_{0}$ brain images for each individual rat were registered to the $b_{0}$ image of one reference rat brain (8 weeks, control group) with linear (Jenkinson and Smith, 2001; Jenkinson et al., 2002) and non-linear transformations (Andersson et al., 2007). The mean $b_{0}$ image of all control rat brains was subsequently registered linearly and nonlinearly to a 3D reconstruction of the Paxinos and Watson rat brain atlas (Majka et al., 2012; Paxinos and Watson, 2005), with some manual refinement to the transformed internal capsule and corpus callosum.

To select the left and right CST from the 250.000 streamlines, inverse transformations were applied to register the bilateral internal capsule and striatum (consisting of caudate putamen and globus pallidus atlas regions) back from reference space to individual animal space (Jenkinson and Smith, 2001; Jenkinson et al., 2002). Additionally, bilateral brain stem (approximately $-7 \mathrm{~mm}$ posterior from bregma) and optic fiber (approximately $-1.5 \mathrm{~mm}$ from bregma) regions were manually outlined for each individual animal, which were used to include or exclude streamlines, respectively. The internal capsule also served to include streamlines in the CST selection process. Only streamlines that passed the brain stem and internal capsule were included. In many rats, some streamlines deviated and bended away from the main bundle towards the optic tract. These streamlines were excluded using a manually drawn exclusion area. Next, parts of streamlines that went through the striatum were excluded (i.e. masked out) for further analysis. As a last step, spurious 'sprouting' streamlines were manually removed from the CST segmentations (Figure S1).

To select the anterior transcallosal tracts, which contain interhemispheric connections between sensorimotor regions, we manually extended the bilateral primary motor (M1) regions in reference space (ventral direction only), resulting in $\mathrm{M} 1$ regions that include underlying white matter. Inverse transformations were applied to register the extended bilateral M1 regions and corpus callosum back from reference space into individual animal space using FLIRT and FNIRT (Jenkinson and Smith, 2001; Jenkinson et al., 2002). We selected all streamlines that passed through the bilateral extended M1 regions and the corpus callosum. Streamlines were cut off at the outside borders of the bilateral M1 regions. The position of the resulting CST and transcallosal tracts are shown as overlays on the lesion incidence map in Figure 1.

Along-tract analysis was performed to obtain the average $F A, M D, A D$ and $R D$ along left and right CSTs as well as the transcallosal tract. In addition, we also determined the number 
of streamlines for the left and right CST, and the transcallosal tract. One DTI dataset of a control rat at 1 week could not be included as it was not saved due to a scanner-related problem. Another DTI dataset of a control rat at 1 week had to be excluded because of bad image quality. Resulting group sizes were $\mathrm{n}_{\text {control }}=6, \mathrm{n}_{\text {stroke }}=8$ (at 1 week), $\mathrm{n}_{\text {control }}=8, \mathrm{n}_{\text {stroke }}$ $=7$ (at 8 weeks) and $n_{\text {control }}=8, n_{\text {stroke }}=7$ (at 28 weeks).

\section{Resting-state functional connectivity}

The first 20 images of the rs-fMRI time-series were discarded to ascertain steady-state. Next, motion correction was performed to the mean image using FSL's MCFLIRT, and motion correction parameters were used for regression of the motion-corrected images using FSL's fsl_glm. The time-series was then bandpass-filtered between 0.01 and $0.1 \mathrm{~Hz}$ using AFNI's 3dFourier tool.

For registration purposes, a mean image of the motion-corrected rs-fMRI time-series was calculated. The resulting mean rs-fMRI image for each rat was corrected for homogeneity and linearly registered to the mean rs-fMRI image of a reference rat brain (control group, 8 weeks). Registered images from all rats were used to calculate a mean rs$\mathrm{fMRI}$ image in reference space, which was used to obtain a brain mask in reference space. The resulting brain mask was inversely transformed to subject space, and applied to the mean rs-fMRI image for each rat in subject space. Rs-fMRI images were subsequently registered to the Paxinos and Watson rat brain atlas (Majka et al., 2012; Paxinos and Watson, 2005), from which we selected 14 regions of interest (ROIs), i.e. the bilateral caudate putamen, $M 1$, secondary motor cortex (M2), forelimb and hindlimb regions of the primary somatosensory cortex (S1FL and S1HL, respectively), secondary somatosensory cortex and thalamus. A temporal-signal-to-noise (tSNR) mask was obtained from the masked motion-corrected rs-fMRI time-series for each rat, by calculating tSNR images and subsequent thresholding of these images at values $\geq 10$. The resulting tSNR mask was applied to the selected ROIs, and seed-based Fisher's z-transformed functional connectivity maps and matrices were calculated from the motion-corrected and regressed rs-fMRI timeseries for each rat.

Seed-based functional connectivity maps with contralesional forelimb or hindlimb regions of the primary somatosensory cortex as seed regions were used to assess data quality. Four datasets were excluded because of major artifacts in the functional connectivity maps (one stroke animal at week 1 , three control animals at week 8 ). One additional dataset could not be included as it was not saved due to a scanner-related problem (control week 1 ). Resulting group sizes were $n_{\text {control }}=6, n_{\text {stroke }}=7$ (at 1 week), $n_{\text {control }}$ $=5, \mathrm{n}_{\text {stroke }}=7$ (at 8 weeks) and $\mathrm{n}_{\text {control }}=8, \mathrm{n}_{\text {stroke }}=7$ (at 28 weeks). 


\section{Sensorimotor function test}

Animals were placed in a transparent Perspex vertical cylinder $(20 \mathrm{~cm}$ diameter, $30 \mathrm{~cm}$ height), which was positioned on a horizontal elevated transparent Perspex plate. Videos of the animal were acquired with a video camera (Panasonic HC V520) located underneath the cylinder. We aimed to record 20 paw placements at minimum.

Videos were analyzed with BORIS software, by an observer blinded to the experimental group and time point. Paw placement was scored as described by Hua et al., 2002 (Hua et al., 2002). Each time the animal reared from a sitting position, or when starting in upright position without touching the cylinder wall, subsequent placement of the entire forelimb(s) against the cylinder wall were scored as paw placement 'left', 'right' or 'both' for independent or simultaneous use of the left and right forelimb, respectively. Touching the cylinder wall with digits only was not scored as paw placement. When the rat was in upright position, each time one paw was horizontally repositioned on the cylinder wall, directly followed by repositioning of the second paw on the cylinder wall, placement was scored as 'both'. In case the second paw was not repositioned or removed from the cylinder wall it was not scored as paw placement.

For each animal, the first 20 paw placement scores were included for calculation of the forelimb-use asymmetry score. Subsequently, sum scores were calculated for the paw placements, resulting in a total paw placement score for 'left' $\left(\mathrm{PP}_{\text {left }}\right)$, 'right' $\left(\mathrm{PP}_{\text {right }}\right)$ and 'both' ( $\left.\mathrm{PP}_{\text {both }}\right)$. Forelimb-use asymmetry, expressed as the percentage usage of the left (impaired) forelimb (LF), was calculated according to the following equation:

$$
L F=\left(\left(\frac{\left(P P_{\text {left }}+P P_{\text {both }} / 2\right)}{\left(P P_{\text {left }}+P P_{\text {right }}+P P_{\text {both }}\right)}\right)\right) \times 100 \%
$$

\section{Statistical analyses}

All statistical analyses and visualization were performed in $R 3.2$ (http://www.rproject.org/), using the packages ggplot2, plyr and reshape2. We used two-way ANOVA to test changes over time and differences between groups (i.e. stroke versus controls) in behavior, diffusion parameters and functional connectivity. Post-hoc analysis was performed using the post-hoc Tukey test. We used Pearson's correlation coefficient to determine the relationship between variables. All statistical tests were corrected for multiple comparisons using the false discovery rate (FDR) method. Results with a FDRcorrected $p$-value $<0.05$ were regarded as significant. For correlation analyses with behavioural measurements we used the average of post-stroke day 1 and week 1 data as first post-stroke behavioural performance score. 


\section{RESULTS}

\section{Brain lesion}

Unilateral cortical lesions were evident on anatomical MRI scans at one week post-stroke. A lesion incidence map is depicted in Figure 1, showing the limited inter-subject variance of the lesion location. The mean ( \pm SD) lesion volume was $33.26 \pm 11.72 \mathrm{~mm}^{3}$, which covered the sensorimotor cortex, with no or minimal involvement of underlying white matter.
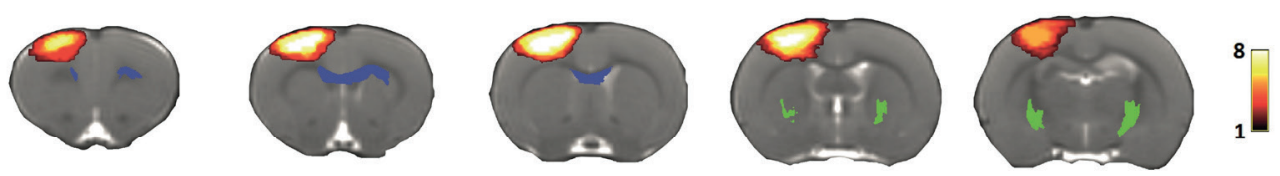

Figure 1. Lesion incidence map and segmented tracts overlaid on anatomical rat brain images. Anatomical rat brain images of coronal slices from anterior (left) to posterior (right). Lesion incidence is scaled from 1 (dark red) to 8 (yellow), indicating the number of rats with a lesion in that particular region. The bilateral CST (green) and transcallosal tracts (blue) reconstructions are also shown.

\section{CST and transcallosal tract integrity}

Reconstructions of the segmented transcallosal tracts and CST, displayed in Figure S2 and Figure 1, showed that these tracts were not part of the ischemic lesion.

Figure 2 shows representative segmentations (in controls) of the bilateral CST and transcallosal tracts, their diffusion MRI-based characteristics, and their number of streamlines. We found a significantly lower average FA along streamlines in the ipsilesional CST of stroke rats as compared to control rats $\left(\Delta_{\mathrm{FA}}=-0.044,95 \%\right.$ C.I. $=-0.058--0.030, F_{\text {group }}$ $=39.71, p<0.0001)$. Post-hoc tests showed significant differences between FA over the ipsilesional streamlines in stroke and controls at 8 weeks $\left(\Delta_{\mathrm{FA}}=-0.056,95 \%\right.$ C.I. $=-0.092-$ $0.021, p<0.001)$ and 28 weeks post-stroke $\left(\Delta_{\mathrm{FA}}=-0.042,95 \%\right.$ C.I. $\left.=-0.078--0.010, p<0.05\right)$. In addition, the average RD along streamlines in the ipsilesional CST of stroke rats was significantly higher than in controls $\left(\Delta_{\mathrm{RD}}=0.045,95 \%\right.$ C.I. $=0.025-0.065, F_{\text {group }}=20.32, p<$ 0.0001). Post-hoc tests showed significant differences in RD over the ipsilesional streamlines at 8 weeks $\left(\Delta_{\mathrm{RD}}=0.052,95 \%\right.$ C.I. $\left.=0.001-0.103, p<0.05\right)$ and 28 weeks poststroke $\left(\Delta_{\mathrm{RD}}=0.053,95 \%\right.$ C.I. $\left.=0.002-0.104, p<0.05\right)$. Although not statistically significant, AD over the ipsilesional CST streamlines tended to be lower in stroke rats at 1 week and 8 weeks as compared to controls. MD over the ipsilesional CST was not significantly different between stroke and controls rats. For the contralesional CST and the transcallosal tracts, we did not find significant differences over time, or between stroke and control rats.

In stroke rats the number of CST streamlines in the ipsilesional (right) hemisphere was significantly lower than the number of CST streamlines in the contralesional (left) hemisphere $\left(\Delta \mathrm{CST}_{\text {streamlines }}=-556,95 \% \mathrm{C} . \mathrm{I} .=-880--232, F_{\text {side }}=11.67, p<0.01\right)$, and the 
number of CST streamlines in the left and right CST of control rats $\left(\Delta \mathrm{CST}_{\text {streamlines }}=-670,95 \%\right.$ C.I. $\left.=-995--347, F_{\text {group }}=16.96, p<0.0001\right)$. The number of streamlines in the corpus callosum $(C C)$ tended to be lower at 28 weeks after stroke $\left(\Delta C C_{\text {streamlines }}=-1082,95 \%\right.$ C.I. $=-$ $2285--112, p=0.10)$.

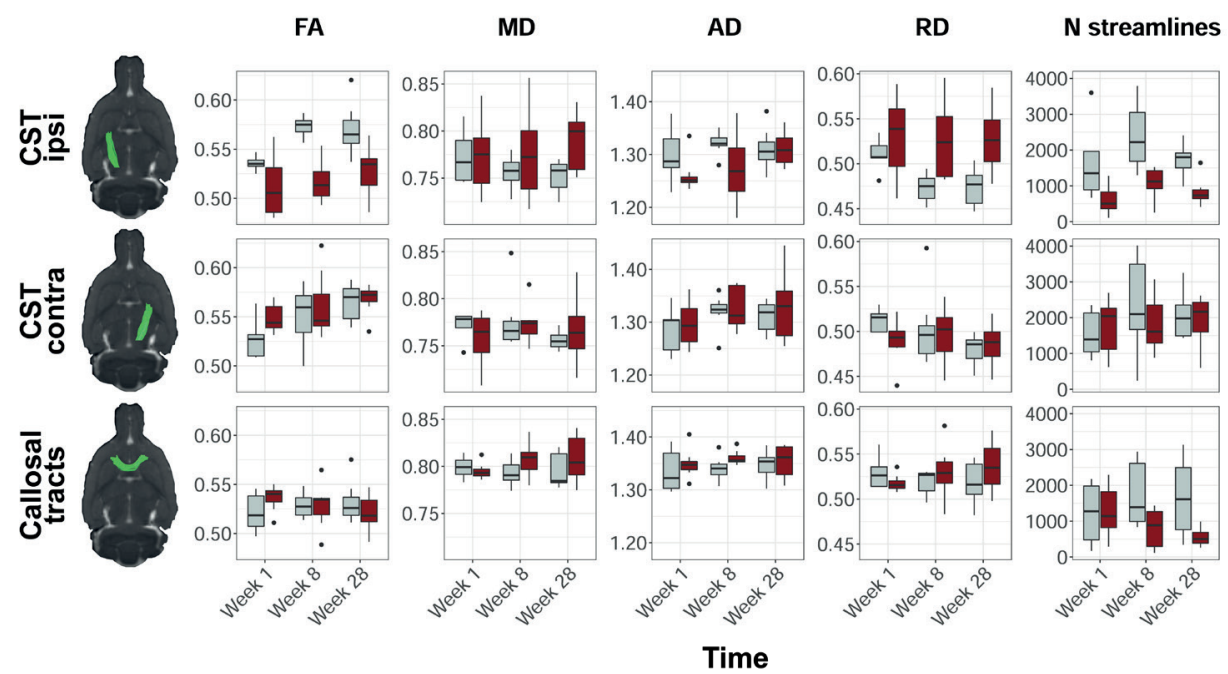

Figure 2. Diffusion MRI-based white matter characteristics in stroke and control rats. Representative segmentations of the ipsilesional CST (top row), contralesional CST (middle row) and transcallosal tracts (bottom row) of a control rat projected on an anatomical rat brain image (sagittal plane). Average fractional anisotropy (FA), mean diffusivity (MD), axial diffusivity (AD), radial diffusivity (RD) and number of streamlines, in control (grey) and stroke (red) rats, at 1 week, 8 weeks and 28 weeks post-stroke are shown for each reconstructed white matter pathway. Boxplots show the median and inter-quartile range (IQR), whiskers represent 1.5 times the IQR, and dots represent outliers. MD, AD and $\mathrm{RD}$ values are scaled $\left(\times 10^{-3} \mathrm{~mm}^{2} / \mathrm{s}\right)$.

\section{Resting-state functional connectivity}

Figure 3 shows the interhemispheric functional connectivities for the bilateral S1FL and the bilateral M1. Interhemispheric functional connectivity of S1FL was significantly lowered in stroke rats as compared to control rats $(F=8.8, p<0.05)$, which was most evident at week 1 and week 8. A similar trend was found for interhemispheric connectivity of $\mathrm{M} 1(F=3.8, p$ $=0.06)$. Post-hoc tests revealed main group-effects of -0.44 (95\% confidence interval $(\mathrm{Cl})$ : $0.75--0.14, p<0.01)$ and $-0.32(95 \% \mathrm{Cl}:-0.65-0.01, p=0.06)$, respectively. Interhemispheric functional connectivity tended to normalize at 28 weeks post-stroke. Similar patterns were found for the other sensorimotor ROIs (Figure S3). 


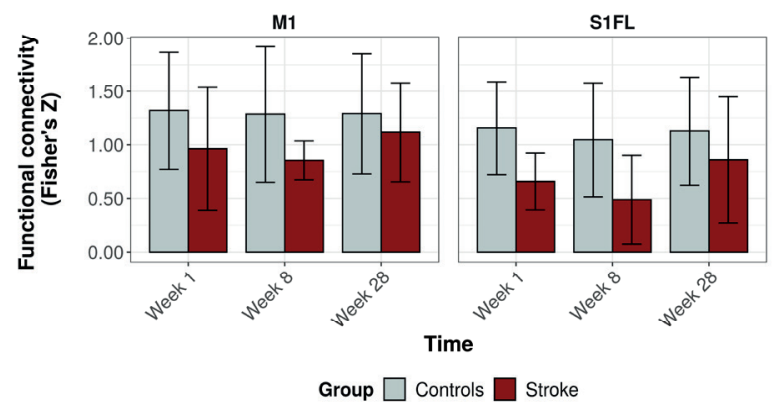

Figure 3. Interhemispheric functional connectivity over time for M1 and S1FL. Functional connectivity as mean ( \pm SD) Fisher's Z for M1 (left) and S1FL (right) at 1 week, 8 weeks and 28 weeks post-stroke (x-axis), for both controls (grey) and stroke rats (red). M1= primary motor cortex; S1FL = forelimb regions of the primary somatosensory cortex.

\section{Functional deficits}

Figure 4 shows the cylinder test results over time for stroke and control rats. We found a significant decline in left (stroke-affected) forelimb usage between stroke and control groups (LF $=-16.2 \%, 95 \%$ C.I. $\left.=-12.4-20.1 \%, F_{\text {group }}=70.3, p<0.0001\right)$. Control rats used their left and right forelimbs about equally over all time points. However, stroke rats showed significant forelimb use asymmetry (predominant usage of the right (unaffected) forelimb) from the acute stages until 8 weeks post-stroke $\left(F_{\text {group } * \text { time }}=3.9, p<0.001\right)$, which normalized during the chronic stages $\left(F_{\text {time }}=5.4, p<0.0001\right)$.

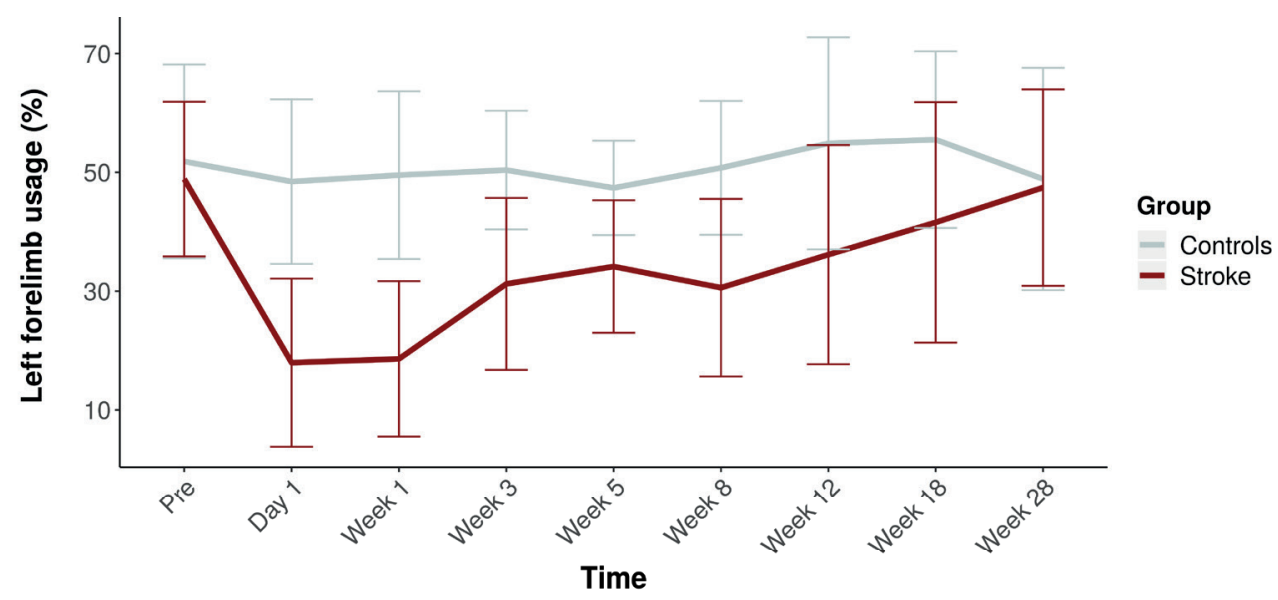

Figure 4. Usage of the left, i.e. stroke-affected, forelimb over time. Mean ( \pm SD) forelimb usage before stroke and at 1 day, and 1, 3, 5, 8, 12, 18 and 28 weeks post-stroke (red). The time-course for controls is shown in grey. 


\section{DISCUSSION}

In the present study we combined longitudinal diffusion MRI, rs-fMRI and behavioral testing to determine to what extent corticospinal and transcallosal tracts are affected in rats recovering from focal cortical stroke, and how this may be linked to changes in functional network connectivity and sensorimotor performance. Diffusion MRI revealed a reduction in the number of streamlines, lower FA values and higher RD values in the ipsilesional CST outside the cortical lesion area. These signs of remote white matter degeneration increased between 1 and 28 weeks post-stroke. The (time-course of) structural features of the transcallosal tracts did not significantly differ between recovering stroke animals and control animals. Resting-state fMRI showed loss of interhemispheric functional connectivity between homologous ipsi- and contralesional sensorimotor cortices at 1 and 8 weeks after stroke, which was partially restored after 28 weeks. This temporal pattern was similar to the time-course of sensorimotor performance changes, which revealed functional recovery after initial loss of forelimb function.

To map structural white matter tracts, we applied an advanced approach of diffusion MRI-based tractography that has been effectively utilized in stroke patient studies (ChiaKoh et al., 2018; Lindenberg et al., 2010, 2013). Recently this technique has also been successfully applied and histologically validated for reconstruction of different white matter tracts in rats (Figini et al., 2015), thereby offering a powerful and unique means for longitudinal in vivo assessment of the large white matter tracts in rodents at whole-brain level. In the present study, we extended this method by developing a pipeline for mapping, segmenting and analyzing the bilateral CST from the rat brain.

So far, the integrity of the CST has hardly been investigated in pre-clinical stroke studies, despite its known significance in (loss and recovery of) sensorimotor function. The reduced number of streamlines that we found in the ipsilesional CST after ischemic cortical damage in rats is in line with recent diffusion MRI studies in human stroke patients, reporting on reduced structural connectivity (strength) in the ipsilesional CST (Cheng et al., 2019a; Lindenberg et al., 2010; Ruber et al., 2012). Furthermore, across all time points we measured reduced FA in the ipsilesional CST of stroke rats as compared to controls. This has also been described in earlier stroke studies in rats and human patients (Lindberg et al., 2007; Lindenberg et al., 2010, 2013; van Meer et al., 2012; Schaechter et al., 2010; Tuor et al., 2014), and is believed to reflect anterograde (Wallerian) degeneration or extensive white matter edema. An increase in RD, also evident in our data, has previously been related to demyelination of white matter tracts (Song et al., 2005; Sun et al., 2006; Tuor et al., 2014). Because the segmentations of the CST were located entirely outside the lesioned territory, the observed structural changes are believed to be due to remote degeneration of fiber tracts that are structurally connected to the infarcted cortex. Noteworthy, in the CST of control rats, FA tended to increase over time, which has previously been associated with 
ongoing white matter maturation in rats from early to late adulthood (Blockx et al., 2011; Mengler et al., 2014). As such, the larger difference in FA along the ipsilesional CST between control rats and stroke rats in the chronic phase, may also be indicative of disturbed white matter maturation.

Despite the indication of remotely degenerating corticospinal fibers after cortical ischemic injury, our study did not reveal similar signs of degeneration of transcallosal tracts, which are known to also arise from the sensorimotor cortex. Although speculative, we think that recruitment of intact contralesional homotopical cortical regions, which may play a compensatory role in sensorimotor recovery (van Meer et al., 2010b, 2012), and sustained interhemispheric neuronal communication through the transcallosal tracts, may counteract or limit secondary white matter degeneration in these tracts. In contrast, decreased usage of the contralateral forelimb, and consequent reduced axonal in- and output may enhance the neurodegenerative processes of the ipsilesional CST (i.e. "use it or lose it") (Allred et al., 2014; Zeiler and Krakauer, 2013). Preservation of transcallosal tracts may also have contributed to the recovery of functional connectivity between ipsi- and contralesional regions of the sensorimotor cortex chronically after stroke. This is in agreement with an earlier study, in which we showed restoration of interhemispheric functional connectivity at 10 weeks after unilateral transient middle cerebral artery occlusion in rats, following initial loss of function during the first three weeks, together with intact trans-hemispheric neuroanatomical connectivity (van Meer et al., 2010b). Moreover, improved sensorimotor performance was related to normalization of interhemispheric functional connectivity (van Meer et al., 2010b), a finding that has also been reported in human stroke studies (Carter et al., 2010, 2012b).

Our current study shows that despite remote CST degeneration, recovery of sensorimotor function is possible in parallel with normalization of interhemispheric functional connectivity, which may strongly rely on the integrity of transcallosal tracts. In addition, other mechanisms, like cortical remapping (Dijkhuizen et al., 2002; Jaillard et al., 2005; Okabe et al., 2016; Ward et al., 2003) or increased involvement of other white matter tracts, such as the rubro-spinal tract (Esposito et al., 2014; Ruber et al., 2012; Takenobu et al., 2014) and reticulospinal tract (Bachmann et al., 2014; Zaaimi et al., 2012), may contribute to alleviation of functional impairments. Future research could employ recently developed quantitative diffusion MRI-based approaches, which enable calculation of fiber density and fiber cross-section (Jeurissen et al., 2014; Raffelt et al., 2012, 2015; Tournier et al., 2011), for improved assessment of white matter characteristics in relation to (potential for) stroke recovery. This may lead to the development of novel diagnostic imaging markers that could better predict functional outcome in patients recovering from stroke and guide individualized treatment strategies. 



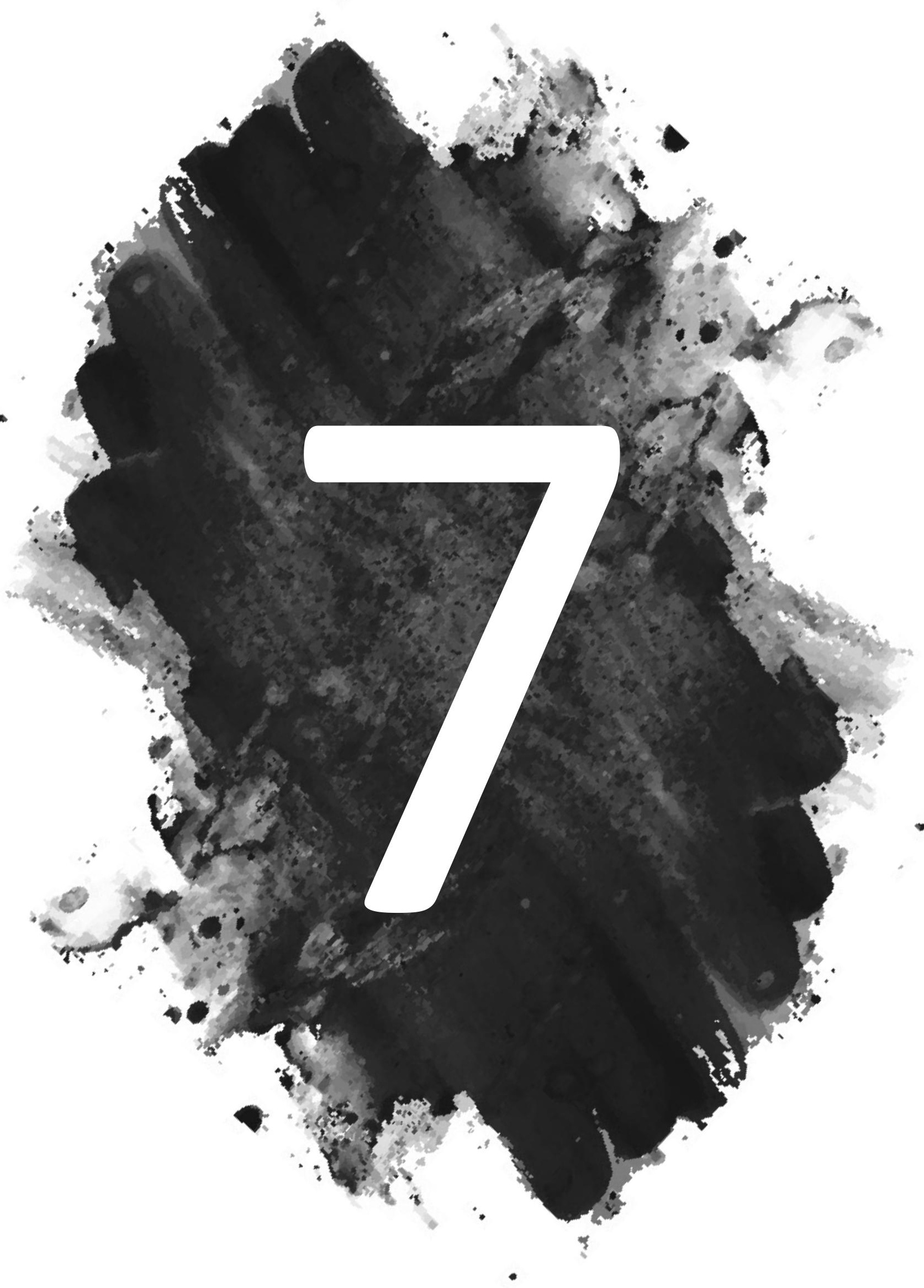




\section{Chapter 7}

\section{Imaging Markers for the Characterization of Gray and White Matter Changes from Acute to Chronic Stages after Experimental Traumatic Brain Injury}

M.R.T. Sinke, W.M. Otte, A.E. Meerwaldt, A.A. Franx, M.H.M. Ali, F. Rakib, A. van der Toorn, C.L. van Heijningen, C.E. Smeele, T. Ahmed, E.L.A. Blezer \& R.M. Dijkhuizen 


\section{ABSTRACT}

Traumatic brain injury (TBI) is a complex pathological process, which involves primary and secondary disease mechanisms. Despite clinical symptoms, a large majority of people with mild TBI - that is, trauma with less than 30 minutes loss of consciousness - has normal CT and MRI scans. As such present-day diagnostic imaging tools are insufficient to diagnose or classify low grades of TBI. Advanced neuroimaging techniques, such as diffusion-weighted MRI and functional MRI, may yield novel biomarkers that may aid in the diagnosis of TBI. Therefore, the present study had two aims. First, to characterize the development of MRIbased measures of structural and functional changes in grey and white matter regions from acute to chronic stages after mild and moderate TBI. Second, to identify imaging markers that can most accurately predict outcome after TBI. To these aims, fifty-two rats underwent serial functional (resting-state blood oxygenation level-dependent (BOLD)) and structural $\left(\mathrm{T}_{1^{-}}, \mathrm{T}_{2^{-}}\right.$, and diffusion-weighted) MRI before and 1 hour, 1 day, 1 week, 1 month and 3-4 months after mild or more severe TBI induction. In addition, all rats underwent behavioral testing, and histology was performed in subgroups of rats at different time-points. We found that early after moderate TBI, axial and radial diffusivities were increased, and fractional anisotropy was reduced in the corpus callosum and bilateral hippocampi. These white matter diffusion parameters normalized over time, which was paralleled by recovery of sensorimotor function after initial loss. Correspondingly, histology revealed decreased myelin staining early after TBI, which was not detected at chronic stages. No significant changes in individual outcome measures were detected after mild TBI. However, multivariate analysis showed a significant additive contribution of diffusion parameters in the distinction between control and different grades of TBI-affected brains. Therefore, combining multiple imaging markers may increase the sensitivity for TBI-related pathology, which may in turn improve the diagnosis of patients with mild TBI symptoms. 


\section{INTRODUCTION}

Traumatic brain injury (TBI) is a heterogeneous disorder, which is characterized by damage to the brain caused by an external mechanical force (Maas et al., 2008; Xiong et al., 2013). This mechanical force may be a consequence of a direct impact, penetration by a projectile, crush, blast wave or rapid acceleration and deceleration, often caused by work-, sport- or traffic-related accidents (Maas et al., 2008; Xiong et al., 2013). TBI can lead to temporary or permanent cognitive impairment as well as physical and psychosocial dysfunction (Maas et al., 2008), and is the leading cause of acquired disability and death in young adults up to 45 years (Langlois et al., 2009; Roozenbeek et al., 2013). TBI is a serious health problem that affects an estimated 5.3 million people in the USA (Langlois et al., 2009) and approximate 7.7 million people in the European Union (Roozenbeek et al., 2013; Tagliaferri et al., 2006). Worldwide, TBI leads to more than 10 million annual deaths and hospitalizations (Langlois et al., 2009; Roozenbeek et al., 2013).

TBI has a complex disease process, involving primary and secondary injury mechanisms rather than a single event (Masel and DeWitt, 2010; Xiong et al., 2013). The primary injury is directly caused by the external force leading to tissue deformation. This injury may result in grey and white matter damage, shearing and tearing of axons, neurons and blood vessels, as well as bleeding and edema. This may subsequently lead to secondary injuries developing over minutes to months after the initial event. Secondary injuries consists of pathophysiological cascades of events, such as ischemia, blood-brain barrier (BBB) damage, excitotoxicity, mitochondrial damage, inflammatory processes (Andriessen et al., 2010; Bains and Hall, 2012; Masel and DeWitt, 2010; Xiong et al., 2013). Together this may result in more tissue damage, diffuse axonal injury, cell death and atrophy (i.e. ongoing neurodegeneration) (Masel and DeWitt, 2010; Xiong et al., 2013). Moreover, these secondary events ultimately lead to functional deficits, such as cognitive impairment (e.g. attention deficits and poor executive functioning), behavioral problems (e.g. aggression) as well as long-term increased vulnerability to other physical, neurological and psychiatric disorders, like sexual dysfunction, epilepsy, sleep and mood disorders (Masel and DeWitt, 2010; Xiong et al., 2013). Although patients may recover over time, many patients continue to suffer from symptoms one year post-injury (McMahon et al., 2013).

Today TBI is primarily diagnosed and classified based on the patients' self-report of clinical symptoms, often with support of the Glasgow Coma Scale (GCS). The diagnosis also includes the duration of the loss of consciousness and the duration of post-trauma amnesia (Borg et al., 2004; Maas et al., 2008). In addition, conventional imaging modalities, such as computed tomography (CT) and magnetic resonance imaging (MRI), are used to map potential structural brain damage, like diffuse injuries, lesions and hematomas (Borg et al., 2004; Maas et al., 2008). Based on the clinical symptoms alone, about $80 \%$ of the patients is classified as suffering from mild TBI (Belanger et al., 2007). However, despite the clinical 
symptoms, a large majority (i.e. up to 75\%) of mild TBI patients has normal CT scans (Belanger et al., 2007; Borg et al., 2004). Even with MRI, which may be more sensitive in detecting tissue injuries than $\mathrm{CT}$, structural scans are normal in $43-68 \%$ of symptomatic patients (Belanger et al., 2007; Borg et al., 2004; Hofman et al., 2001; Hughes et al., 2004). Hence, present-day conventional diagnostic imaging tools are not sufficient to properly diagnose or classify different grades of TBI and to predict its post-injury functional outcome. Improved spatiotemporal characterization of post-injury mechanisms after different grades of brain injury may ultimately improve TBI diagnosis, classification and prognosis. Advanced neuroimaging techniques may yield novel biomarkers that can depict tissue status and outcome with more precision. MRI techniques, such as diffusion MRI, diffusion tensor imaging (DTI) and blood oxygenation-level dependent (BOLD) resting-state functional MRI ( $r$-fMRI), have shown promise in detecting distinctive biomarkers of structural and functional brain damage in pre-clinical and clinical research settings. Diffusion MRI is based on the random motion (i.e. diffusion) of water molecules, which is influenced by the brain tissue's architecture, and specifically the alignment of myelinated axonal fibers. Hence, diffusion MRI parameters can be used to indirectly probe the underlying white matter structure and its integrity (Basser et al., 1994a). Diffusion parameters, such as fractional anisotropy (FA), and mean, axial and radial diffusivity (resp. MD, AD and RD), have been shown to be altered in the brains of human TBI patients (Bazarian et al., 2007; Edlow et al., 2016; Inglese et al., 2005; Ling et al., 2012; Mayer et al., 2010; Palacios et al., 2012; Rutgers et al., 2008) and animals with experimental TBI (Braeckman et al., 2019; Li et al., 2017a, 2013; Long et al., 2015; van de Looij et al., 2012; Singh et al., 2016; Tu et al., 2016; Wang et al., 2018; Wright et al., 2016; Zhuo et al., 2012), which has been histologically related to axonal damage (Braeckman et al., 2019; Li et al., 2017b, 2013; Singh et al., 2016; Zhuo et al., 2012). Furthermore, with resting-state fMRI altered functional connectivity has been measured in TBI patients' brains (Hillary et al., 2011). Early loss followed by subsequent (partial) recovery of functional connectivity has been described in a rat TBI model (Heffernan et al., 2013). Thus, in addition to conventional $\mathrm{T}_{1}$ - or $\mathrm{T}_{2}$-weighted MRI (with or without contrast agent) to detect tissue lesions, diffusion and functional MRI techniques may aid in the diagnosis of the complex heterogeneous disease process of TBI.

Therefore, the present study aimed $(a)$ to characterize the development of structural and functional changes in grey and white matter regions from acute to chronic stages after mild and moderate TBI and $(b)$ to identify imaging markers that can most accurately predict outcome after TBI. To that aim we longitudinally acquired $\mathrm{T}_{2}$-weighted $\mathrm{MRI}$, post-contrast $\mathrm{T}_{1}$-weighted $\mathrm{MRI}$, diffusion $\mathrm{MRI}$ and resting-state functional $\mathrm{MRI}$ in a straightforward and well-controlled animal model for different degrees of TBI, i.e. the Marmarou weight-drop model (Foda and Marmarou, 1994; Marmarou et al., 1994). In addition, serial behavioral 
testing and post mortem histology were executed to relate MRI findings to sensorimotor function and microstructural tissue status, respectively.

\section{METHODS}

\section{Animals and protocol}

All animal procedures and experiments were approved by the Animal Experiments Committee of the University Medical Center Utrecht and Utrecht University, and were performed in accordance with the guidelines of the European Communities Council Directive.

The present study was performed in adult male Sprague-Dawley rats ( $N=52$ ) (Charles River Laboratories, Germany), which were housed in pairs under standard conditions with light/dark cycle of 12/12 hours (light on from 07:00 till 19:00), and ad libitum access to food and water.

Serial MRI was performed on a 4.7 T horizontal bore MR (Varian Instruments, Palo Alto, CA, USA) in rats before and 1 hour, 1 day, 1 week, 1 month and 3-4 months after TBI induction. In addition, all rats underwent longitudinal behavioral testing (Figure 1). At every time point a number of rats was sacrificed after the MRI experiments for histological examination (Table 1).

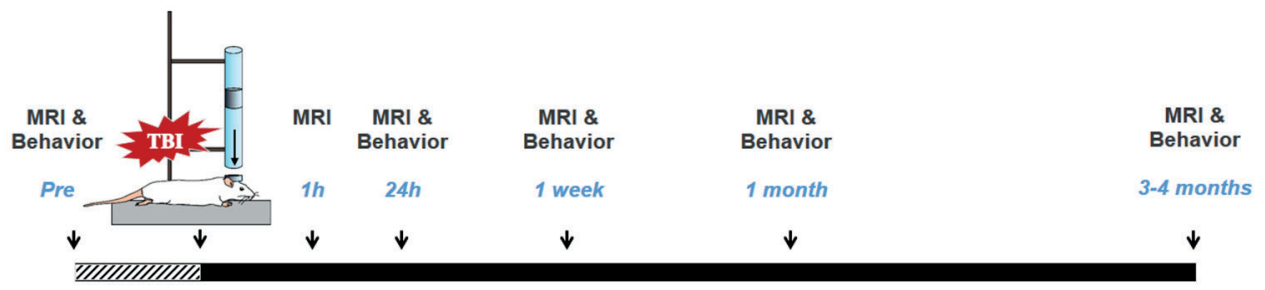

Figure 1. Study timeline. Rats were scanned before traumatic brain injury (TBI), and at 1 hour, 24 hours, 1 week, 1 month and 3-4 months after TBI induction. MRI and behavioral assessment were performed at all time-points, except at the 1 hour post-TBI time-point when animals only underwent MRI. At every time-point a subset of rats was sacrificed for histological assessment (see Table 1 for numbers).

\begin{tabular}{lcccccc}
\hline Group & Pre-TBI & $\mathbf{1}$ hour & $\mathbf{1}$ day & $\mathbf{1}$ week & $\mathbf{1}$ month & ３/4 months \\
\hline Control & $\mathbf{1 3}$ & $\mathbf{1 2}$ & $\mathbf{7}$ & $\mathbf{7}$ & $\mathbf{6}$ & $\mathbf{4}$ \\
& $(1)$ & $(5)$ & $(0)$ & $(1)$ & $(2)$ & $(4)$ \\
\hline Mild TBI & $\mathbf{2 3}$ & $\mathbf{2 1}$ & $\mathbf{1 7}$ & $\mathbf{1 2}$ & $\mathbf{9}$ & $\mathbf{4}$ \\
& $(2)$ & $(4)$ & $(5)$ & $(3)$ & $(5)$ & $(4)$ \\
\hline Moderate & $\mathbf{1 8}$ & $\mathbf{7}^{\mathrm{a}}$ & $\mathbf{1 7}$ & $\mathbf{1 2}$ & $\mathbf{6}$ & $\mathbf{6}$ \\
TBI & $(1)$ & $(0)$ & $(5)$ & $(6)$ & $(0)$ & $(6)$ \\
\hline
\end{tabular}

Table 1. Number of animals per MRI time point. The number between parentheses indicates the number of sacrificed animals for post mortem analyses. ${ }^{a}=10$ out of 17 rats were not scanned 1 hour post-TBI but were re-included at 1 day post-TBI. 


\section{$T B I$ induction}

TBI was induced when rats weighed approximately 350 gram. Rats were first anaesthetized with $4 \%$ isoflurane for endotracheal intubation, followed by mechanical ventilation with 2.0-2.5\% isoflurane in air $/ \mathrm{O}_{2}$ (4:1) (Sigma, Netherlands). Rats received a subcutaneous injection of $0.05 \mathrm{mg} / \mathrm{kg}$ buprenorphine (Temgesic, Schering-Plough, Netherlands) for postsurgical pain relief. Body temperature was maintained at $37.0 \pm 0.5^{\circ} \mathrm{C}$.

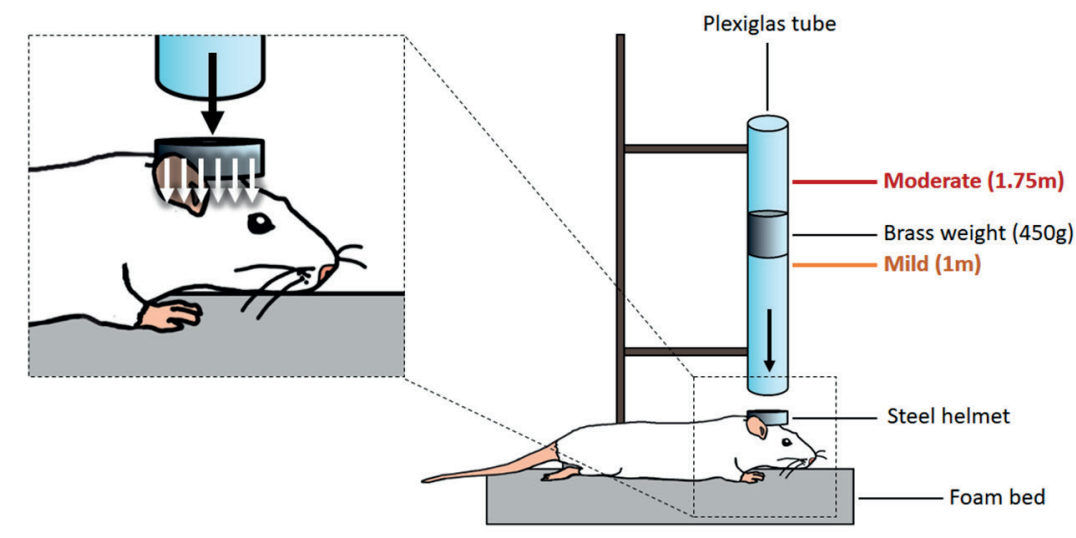

Figure 2. Schematic representation of the Marmarou weight-drop model. The Marmarou weightdrop model induces diffuse axonal injury by a trauma device, consisting of a $450 \mathrm{~g}$ brass weight that falls freely from a specific height through a Plexiglas tube (i.e. from 1 or 1.75 meter to induce mild or moderate TBI, respectively). A stainless steel disc is mounted to the skull at a midline position to prevent skull fracture.

TBI was induced according to the Marmarou weight-drop model (Foda and Marmarou, 1994; Marmarou et al., 1994) (Figure 2). First, a steel disk was mounted on the head. The disk prevented skull fractures by distributing the force of the impact equally over the skull. Subsequently, animals were positioned on a foam bed, below the Plexiglas tube, after which a brass weight (450 gram) was dropped on their head, which causes a fast acceleration/deceleration event. Mild TBI was induced by a weight drop from 1 meter, whereas moderate TBI was induced by a weight drop from 1.75 meter (i.e. larger impact). Immediately after the impact, rats were removed from the foam bed and the steel disk was removed from the head.

\section{Behavioral testing}

Sensorimotor deficit score: to examine sensorimotor function we used a series of motor, sensory, reflex and balance tests, and calculated a composite sensorimotor deficit score, ranging from 0-10, which corresponds to no sensorimotor deficits (score: 0 ) to severe sensorimotor deficits (score: 10). This score was modified from Reglodi and colleagues (Reglodi et al., 2003). 
Beam walk test: the beam walk test was used to quantify locomotion and balance (Metz and Whishaw, 2002). The beam walk consisted of a long tapering beam with a support beam placed below. Animals were trained with and without support beam. We measured the time (in seconds) rats needed to cross the beam. In addition, we measured the number, side and location of slips.

\section{MRI acquisitions}

All MRI acquisitions were performed on a 4.7 horizontal bore MR system (Varian Instruments, Palo Alto, CA, USA), with a homebuilt Helmholtz volume coil (90 mm diameter) for signal excitation, and an inductively coupled surface coil (25 mm diameter) for signal detection. Before MRI measurements, rats were anesthetized with $4 \%$ isoflurane for endotracheal intubation, followed by $2.0-2.5 \%$ isoflurane in air/ $\mathrm{O}_{2}$ (4:1) (Sigma, Netherlands). Rats were placed in a plastic holder and stabilized with a tooth holder and earplugs. When rats were completely anesthetized, they received a subcutaneous injection of $0.05 \mathrm{mg} / \mathrm{kg}$ glycopyrrolate (Robinul, Riemser, Germany) to reduce salivary, tracheobronchial and pharyngeal secretions. During the MRI measurements heart rate and blood oxygen saturation were monitored, and body temperature was maintained at $37.0 \pm 0.5^{\circ} \mathrm{C}$.

Anatomical imaging was performed with a balanced steady-state free precession (BSSFP) sequence with an isotropic voxel resolution of $250 \mu \mathrm{m}$ (repetition time (TR) $=5 \mathrm{~ms}$, echo time $(T E)=2.5 \mathrm{~ms}$, flip angle $=20^{\circ}$, field-of-view $(F O V)=40 \times 32 \times 24 \mathrm{~mm}^{3}$, and data matrix size $=160 \times 128 \times 96)$.

Multi-echo, multi-slice $\mathrm{T}_{2}$-weighted MRI was performed with a 4-shot 3D echo planar imaging (EPI) sequence (TR/TE spacing=350/17, 20, 25, 30, 40, 50, 60, 80, 100 ms; echo train length = 9; acquisition matrix = $92 \times 96 \times 38$; voxel dimensions $=350 \times 350 \times 500 \mu^{3}$ ). Diffusion-weighted imaging was performed using a 4-shot 3D EPI sequence, with TR/TE = $350 / 27 \mathrm{~ms}, \Delta / \delta 12.3 / 6 \mathrm{~ms}$ and $\mathrm{b}$-value $=1280 \mathrm{~s} / \mathrm{mm}^{2}$. FOV was $32.2 \times 33.6 \times 19 \mathrm{~mm}^{3}$ and data matrix size was $92 \times 96 \times 38$, resulting in a voxel resolution of $350 \times 350 \times 500 \mu^{3}$. The protocol consisted of acquisition of five non-diffusion-weighted $\left(b_{0}\right)$ images and 60 images with different non-collinear diffusion-weighting directions on a half sphere.

Resting-state $\mathrm{fMRI}$ was performed using a multi-shot 3D gradient-echo EPI sequence with an isotropic spatial resolution of $600 \mu \mathrm{m}\left(\mathrm{TR} / \mathrm{TE}=38.4 / 20 \mathrm{~ms}\right.$, flip angle $=14^{\circ}$, image repetition time $=954.8 \mathrm{~ms}, \mathrm{FOV}=32.4 \times 32.4 \times 16.8 \mathrm{~mm}^{3}$, matrix $=54 \times 54 \times 28,1$ average, 800 images, total scan time ca. $10 \mathrm{~min}$ ).

$\mathrm{T}_{1}$-weighted images were acquired with a $3 \mathrm{D}$ spoiled gradient echo imaging sequence $\left(\mathrm{TR} / \mathrm{TE}=6.5 / 2.06 \mathrm{~ms}\right.$, flip angle $=40^{\circ}, 2$ averages, data matrix $=92 \times 96 \times 38$, voxel dimensions $=350 \times 350 \times 500 \mathrm{\mu m}^{3}$ ), before and 30 minutes after injection of $0.33 \mathrm{mmol} / \mathrm{kg}$ gadobutrol (Gadovist, Bayer, Germany) in a tail vein. 


\section{MRI data processing and registration}

All images were processed with FSL 5.0 (http://ww.fmrib.ox.ac.uk/fsl/) (Jenkinson et al., 2012). For all images brain extraction was performed with FSL's Brain Extraction Tool (BET) (Smith, 2002). Images were corrected for inhomogeneities and were registered to a reference image of the rat brain using the FSL Linear Image Registration Tool (FLIRT) (Jenkinson and Smith, 2001; Jenkinson et al., 2002) and FSL Non-linear Image Registration Tool (FNIRT) (Andersson et al., 2007). The reference image was matched with a custom built $3 \mathrm{D}$ reconstruction of the Paxinos and Watson rat brain atlas (Majka et al., 2012; Paxinos and Watson, 2005). We assessed the following regions of interest (ROIs) (left and right): the caudate putamen ( $\mathrm{CPu})$, hippocampus $(\mathrm{Hc})$, primary motor cortex (M1), forelimb region of the primary somatosensory cortex $(\mathrm{S} 1 \mathrm{fl})$ - the latter two were merged to form 'sensorimotor cortex' for diffusion MRI analyses -corpus callosum (CC) and internal capsule (IC) (Figure 3). All ROIs were back-projected into subject space. For all images subsequent analyses and data extractions were performed in subject space.

After preprocessing and registration of $\mathrm{T}_{2}$-weighted images, we calculated quantitative $\mathrm{T}_{2}$ maps from a weighted linear least squares fit of the logarithm of the signal intensity versus the echo time, on a voxelwise basis. Subsequently, average $T_{2}$-values were extracted from all ROls.

After preprocessing and registration of diffusion-weighted images, FSL's DTIFIT was used to calculate fractional anisotropy (FA), mean diffusivity (MD), axial diffusivity (AD) and radial diffusivity (RD) maps in subject space. Subsequently, average $F A, M D, A D$ and $R D$ values were extracted from all ROIs.

To ensure steady state, the first twenty images of the rs-fMRI scans were removed. Subsequently, the remaining images were motion-corrected to the mean volume with MCFLIRT (Jenkinson et al., 2002) and brain-extracted with BET (Smith, 2002). An average of the motion-corrected and extracted brain was used for registrations to the reference space and atlas (in a similar manner as described above). The resulting motion correction parameters were used as regressors for the resting-state signal. No global signal regression was applied. Subsequently, low-frequency BOLD fluctuations were obtained from the resting-state data by band-pass filtering between 0.01 and $0.1 \mathrm{~Hz}$ using AFNI (Cox, 1996). Fisher's Z-transformed correlation coefficients were calculated between the average timeseries for all pairs of ROIs. For the $\mathrm{Hc}, \mathrm{CPu}, \mathrm{M} 1$ and $\mathrm{S} 1 \mathrm{fl}$, interhemispheric functional connectivity was determined between left and right homotopic counterparts. Intrahemispheric functional connectivity was determined between CPu and S1fl, CPu and $\mathrm{M} 1$, and S1fl and M1. For each pair of regions (inter- and intrahemispheric) we determined the average inter-regional Fisher's Z-transformed correlation per group ('control', 'mild TBI' and 'moderate $\mathrm{TBI}^{\prime}$ ') per time point. For the intrahemispheric functional connectivities, we 
used the average Fisher's Z-transformed correlation over the two hemispheres for each pair of ROls.

BBB leakage was determined from parenchymal accumulation of gadobutrol. This was measured by calculating the percentage change in $T_{1}$-weighted signal between the pre- and post-contrast scan for each ROI.
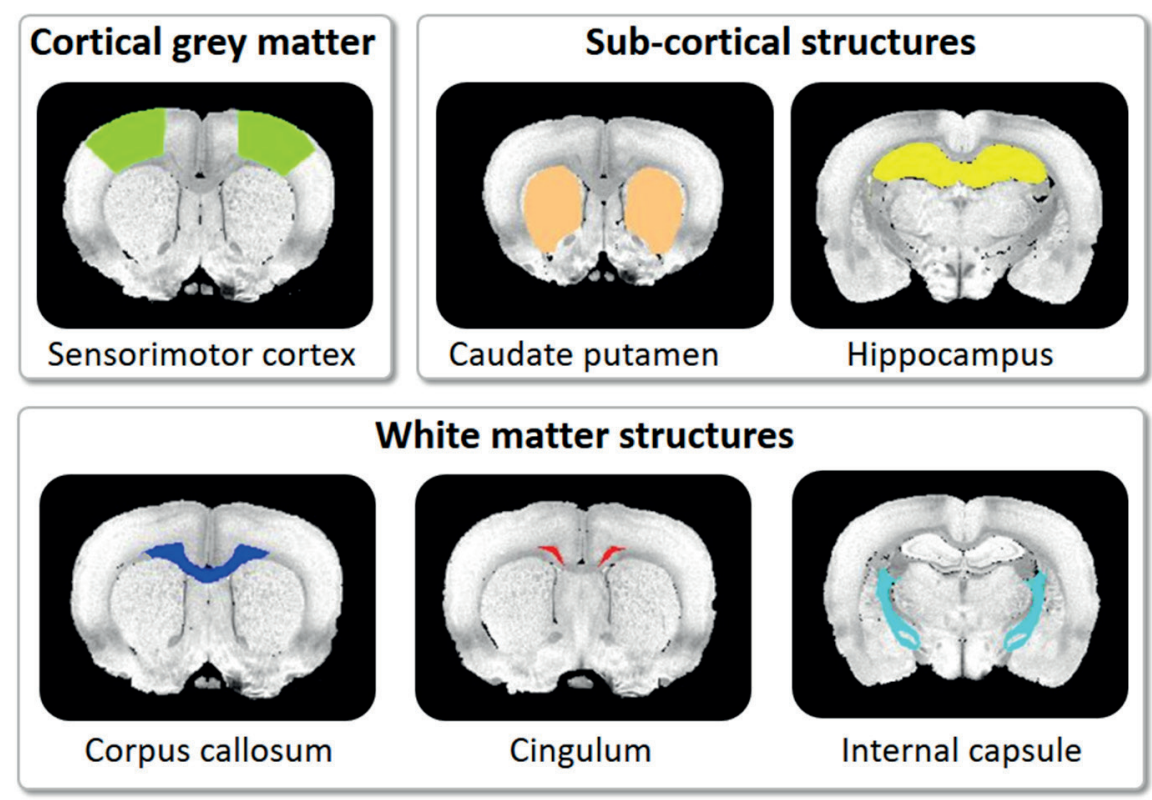

Figure 3. Regions of interest. Bilateral regions of interest (colored areas) are overlaid on highresolution anatomical $\mathrm{T}_{2}$-weighted images of coronal slices from a rat brain template. Top-left: cortical grey matter: sensorimotor cortex, top-right: sub-cortical structures: caudate putamen and hippocampus, bottom: white matter structures: corpus callosum, cingulum bundle and internal capsule.

\section{Immunohistochemistry}

At 24 hours and 3-4 months after TBI induction, animals received an overdose of isoflurane and were transcardially perfused with $0.9 \%$ saline followed by $4 \%$ paraformaldehyde in phosphate-buffered saline (PBS). Brains were post-fixed overnight in $4 \%$ paraformaldehyde and embedded in paraffin. Subsequently $8 \mu \mathrm{m}$ coronal sections were cut on a microtome. Sections were deparaffinized prior to blocking endogenous peroxidases with $3 \% \mathrm{H}_{2} \mathrm{O}_{2}$ in methanol for $20 \mathrm{~min}$, after which the sections were rehydrated.

After rehydration, sections were blocked for non-specific binding of secondary antibody with $20 \% \mathrm{NRbS} / 0.1 \%$ Tween 20 in PBS for $30 \mathrm{~min}$ at $37{ }^{\circ} \mathrm{C}$ before overnight incubation at $4^{\circ} \mathrm{C}$ with primary antibody (Rat-anti-myelin basic protein (MBP), $1: 500$ in $10 \% \mathrm{NRbS} / 1 \%$ Tween 20 in PBS). Sections were washed and incubated with secondary antibody (Rabbit- 
anti-Rat-Biotin 1:400) in PBS/0.1\% Tween 20 (Vector, BA-4000) for $45 \mathrm{~min}$ at room temperature. Subsequently, sections were washed and incubated with $A B$ Complex for 30 $\mathrm{min}$ at room temperature. The sections were washed in $\mathrm{Tris}-\mathrm{HCl}(0.05 \mathrm{M} \mathrm{pH7.6)}$ and stained with 3'-Diaminobenzidine for $10 \mathrm{~min}$, and counterstained with Hematoxylin for $1 \mathrm{~min}$ and embedded in a neutral solution of polystyrene and plasticizers in xylene (DEPEX).

Stained sections containing the corpus callosum, cingulum and cortex were photographed using an AxioLab.A1 microscope equipped with an AxioCam ICC 5 camera (Zeiss) at $2.5 \times$ and $10 \times$ magnification. MBP immunodensity was assessed by measuring the average grey value of each region of interest, subtracted and divided by the average value of the background staining.

\section{Statistical analyses}

Linear mixed-model analysis was performed on diffusion parameter values, functional connectivity values and sensorimotor function scores between groups and time points. The factors group, time and interaction (group by time) were set as fixed effects, whereas subject (i.e. rat) was set as random effect. Post-hoc tests (with Bonferroni corrections) were used to test for differences between groups across different time points as well as between different time points within groups.

Multiple logistic regression analysis was used to evaluate the predictive value of a model combining different MRI parameters at 1 hour and 24 hours after TBI induction, to discriminate between control, mild and moderate TBI rats. We used a step-wise approach and fitted multiple models using different explanatory variables. First a full model (i.e. combining $T_{2}$ and diffusion parameter values; Model 1) was tested against a null-model. At every next step we dropped an explanatory variable from the model and statistically compared the new (nested) model (Models 2-5) with the previous one. The different model fits were evaluated with the McFadden's pseudo $R^{2}$, where values between 0.2 and 0.4 are suggested to represent a very good fit (Louviere et al., 2000). Model comparisons were performed with Akaike Information Criterion (AIC) values and Chi-squared tests. If an explanatory variable was dropped from the model and the explanatory power of the model significantly decreased, this variable was considered of significant value for the model. Vice versa if the explanatory power remained stable or increased, the removed variable was considered insignificant.

All statistical analyses and visualizations were performed in $R 3.4 .3$ (http://www.rproject.org/), using the packages ggplot2, plyr, reshape2, glmmTBM, Ime4, pscl, emmeans and multcomp. 


\section{RESULTS}

\section{Sensorimotor function}

Behavioral tests showed that sensorimotor performance was significantly affected after moderate TBI, but not after mild TBI or in control rats (Figure 4). In moderate TBI rats, the sensorimotor deficit score was significantly increased at 24 hours post-TBI as compared to baseline $(\triangle \mathrm{SDS}=4.4, p<0.0001)$, control rats $(\triangle \mathrm{SDS}=4.8, p<0.0001)$ and mild TBI rats $(\Delta \mathrm{SDS}=4.5, p<0.0001$ ) (Figure 4, left). Also, the number of slips in the beam walk test was significantly increased at 24 hours post-TBI in moderate TBI rats as compared to baseline $\left(\Delta \mathrm{N}_{\text {slips }}=4.6, p<0.001\right)$, control rats $\left(\Delta \mathrm{N}_{\text {slips }}=4.9, p<0.01\right)$ and mild TBI rats $\left(\Delta \mathrm{N}_{\text {slips }}=5.2, p\right.$ $<0.01$ ) (Figure 4, right). Sensorimotor function scores were normalized at one week and later time points.
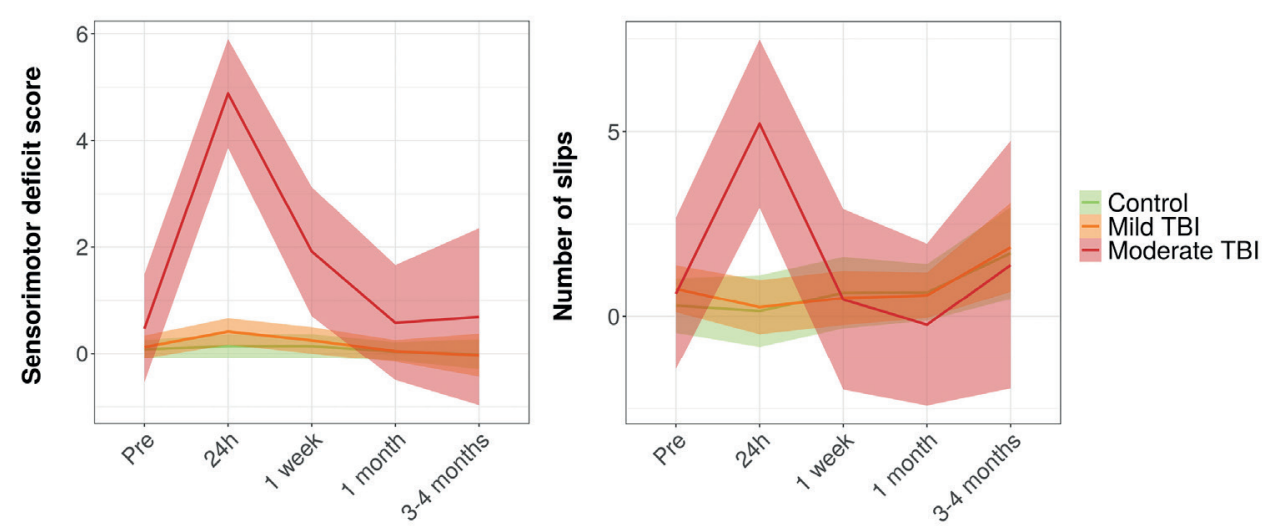

Figure 4. Sensorimotor dysfunction over time. Sensorimotor deficit score (left) and number of slips on the beam walk test (right) shown at different time points for the control (green), mild TBI (orange) and moderate TBI (red) group (mean \pm standard deviation (SD)).

\section{MRI of tissue damage}

Figure 5 shows average FA heatmaps of control, mild and moderate TBI rat brains at 1 hour post-TBI. Analyses of quantitative $\mathrm{T}_{2}$-maps revealed prolonged $\mathrm{T}_{2}$ after moderate TBI in the corpus callosum and hippocampus at 1 hour (CC: $\Delta \mathrm{T}_{2}=0.021, p<0.0001 ; H c: \Delta \mathrm{T}_{2}=0.006, p$ $<0.01$ ) and 24 hours post-TBI (CC: $\left.\Delta \mathrm{T}_{2}=0.012, p<0.0001 ; H c: \Delta \mathrm{T}_{2}=0.006, p<0.0001\right)$ as compared to baseline, at 1 hour as compared to control animals ( $C C: \Delta \mathrm{T}_{2}=0.022, p<0.0001$; $\left.H c: \Delta \mathrm{T}_{2}=0.006, p<0.05\right)$ and mild TBI rats (CC: $\Delta \mathrm{T}_{2}=0.019, p<0.0001 ; H c: \Delta \mathrm{T}_{2}=0.006, p<$ 0.01 ), which normalized at 1 week post-TBI (Figure 6). Diffusion MRI data showed that FA was decreased in these two regions at 1 hour (CC: $\triangle \mathrm{FA}=-0.05, p=0.1$ (Figure 5, bottom); $H c: \triangle \mathrm{FA}=-0.24, p<0.05)$, while $\mathrm{AD}$ and RD were increased at 1 hour (CC: $\triangle \mathrm{AD}=0.015, p<$ $0.001 ; \Delta \mathrm{RD}=0.016, p<0.0001 ; \mathrm{Hc}: \Delta \mathrm{AD}=0.011, p<0.0001 ; \Delta \mathrm{RD}=0.009, p<0.0001)$ and 24 hours (CC: $\triangle \mathrm{AD}=0.014, p<0.01 ; \Delta \mathrm{RD}=0.012, p<0.001 ; H c: \Delta \mathrm{AD}=0.007, p<0.0001$; 
$\Delta \mathrm{RD}=0.005, p<0.001$ ) after moderate TBI (Figure 6). FA, AD and RD values normalized at later time points. In the cingulum, similar effects were found for $A D$ and $R D$ after 1 hour $(\Delta \mathrm{AD}=0.014, p<0.0001 ; \Delta \mathrm{RD}=0.007, p<0.05)$ and 24 hours $(\triangle \mathrm{AD}=0.011, p<0.0001)$, whereas no significant changes in FA were measured. Significant alterations in $T_{2}$ or diffusion parameters were not measured in the other ROIs after moderate TBI. Furthermore, no significant TBI-induced changes over time were observed in any of the ROIs in mild TBI rats. FA in the internal capsule of control rats, however, significantly increased over time $\left(\Delta \mathrm{FA}_{4 \mathrm{~m}-\mathrm{pre}}=0.089, p<0.001\right)$ and was significantly higher at 3-4 months after TBI induction as compared to moderate TBI rats ( $\triangle \mathrm{FA}=0.08, p<0.05)$.

Post-contrast $\mathrm{T}_{1}$-weighted MRI data did not reveal significant signal changes that would indicate parenchymal leakage of the intravenously injected contrast agent (Figure 6).

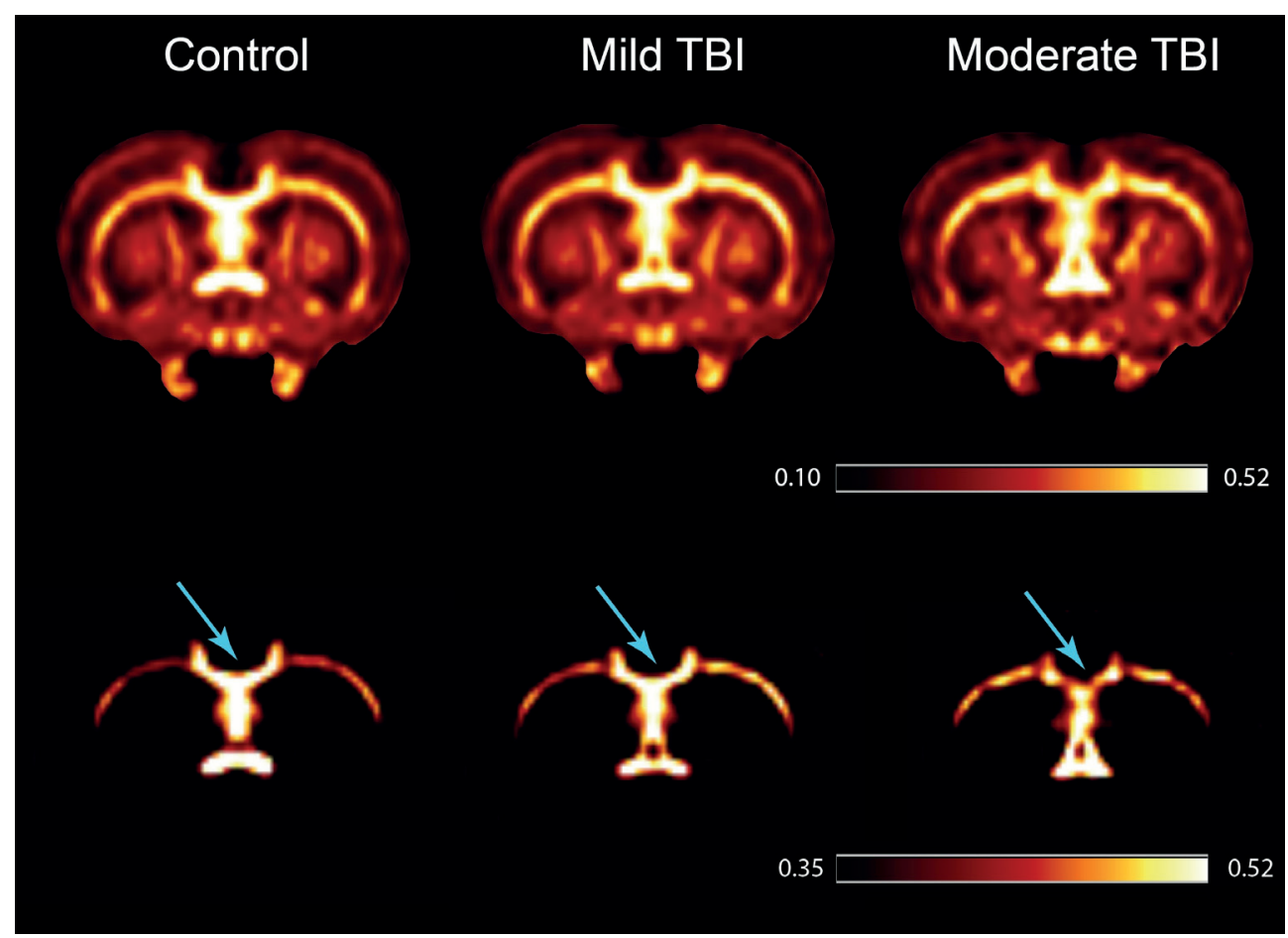

Figure 5. Average FA heatmaps of control and TBI rat brains at $\mathbf{1}$ hour post-TBI. Average FA heatmaps of coronal brain slices showing whole-brain images (top; FA range: 0.10-0.52) and segmented white matter images (bottom; FA range: 0.35-0.52) for control (left), mild TBI (middle) and moderate TBI (right) rats at 1 hour post-TBI. Moderate TBI animals show lower average FA in the corpus callosum (arrows). 

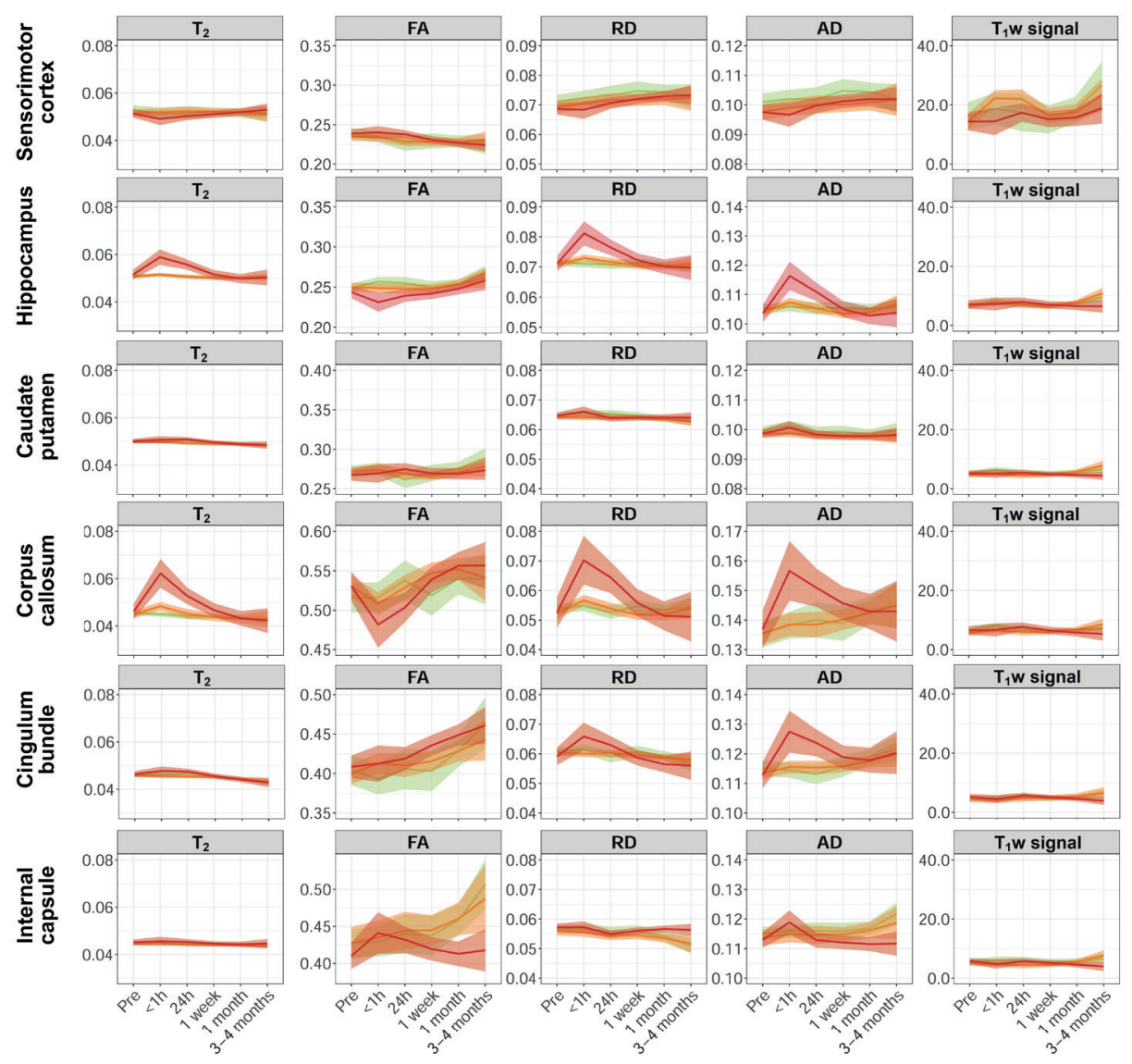

Time

Figure 6. $T_{2}$, diffusion parameters and blood-brain barrier permeability over time. $T_{2}(s)$, fractional anisotropy (FA), radial diffusivity (RD) $\left(10^{-2} \mathrm{~mm}^{2} / \mathrm{s}\right)$, axial diffusivity (AD) $\left(10^{-2} \mathrm{~mm}^{2} / \mathrm{s}\right)$ and gadobutrolinduced $\mathrm{T}_{1}$-weighted signal changes (a.u.) in the sensorimotor cortex, hippocampus, caudate putamen, corpus callosum, cingulum and internal capsule shown over time ( $x$-axis) for the control (green), mild TBI (orange) and moderate TBI (red) group (mean \pm standard deviation (SD)).

\section{MRI of resting-state functional connectivity}

Interhemispheric functional connectivity as measured with rs-fMRI, was overall elevated in moderate $\mathrm{TBI}$ rats as compared to mild TBI and control rats in the bilateral $\mathrm{CPu}(\triangle \mathrm{FC}=0.21$, $p<0.05), \mathrm{Hc}(\Delta \mathrm{FC}=0.21, p<0.05), \mathrm{M} 1(\Delta \mathrm{FC}=0.27, p<0.01)$ and $\mathrm{S} 1 \mathrm{fl}(\Delta \mathrm{FC}=0.29, p<0.01)$, (Figure 7).

Also overall intrahemispheric functional connectivity between subcortical $\mathrm{CPu}$ and sensorimotor cortical regions, $\mathrm{M} 1(\Delta \mathrm{FC}=0.19, p<0.05)$ and $\mathrm{S} 1 \mathrm{fl}(\Delta \mathrm{FC}=0.23, p<0.05)$, as well as between $\mathrm{M} 1$ and $\mathrm{S} 1 \mathrm{fl}(\Delta \mathrm{FC}=0.009, p=0.07)$ was significantly increased in moderate 
TBI rats. No significant changes in functional connectivity were found for mild TBI or control rats.

Interhemispheric Functional Connectivity

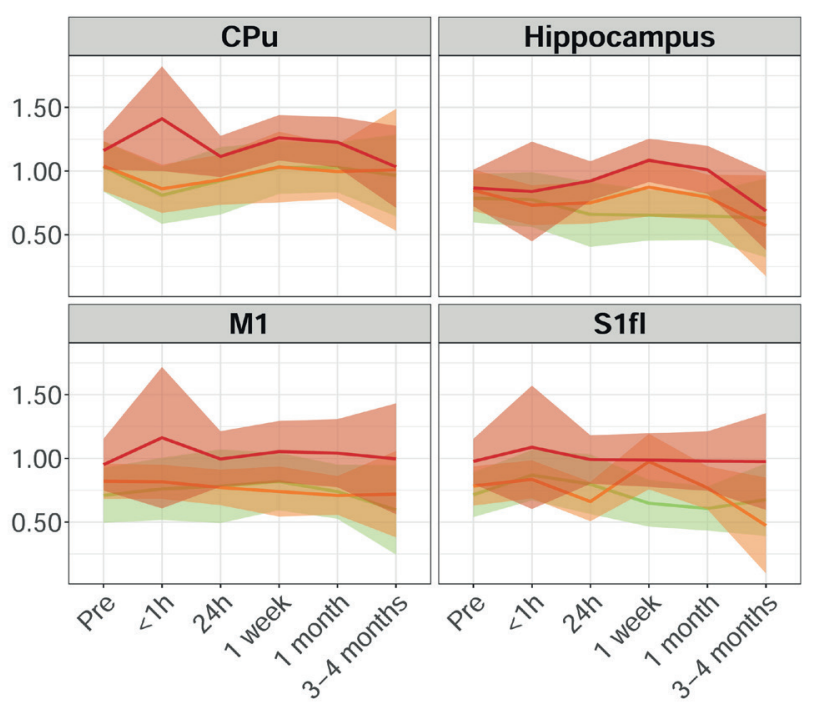

Intrahemispheric Functional Connectivity

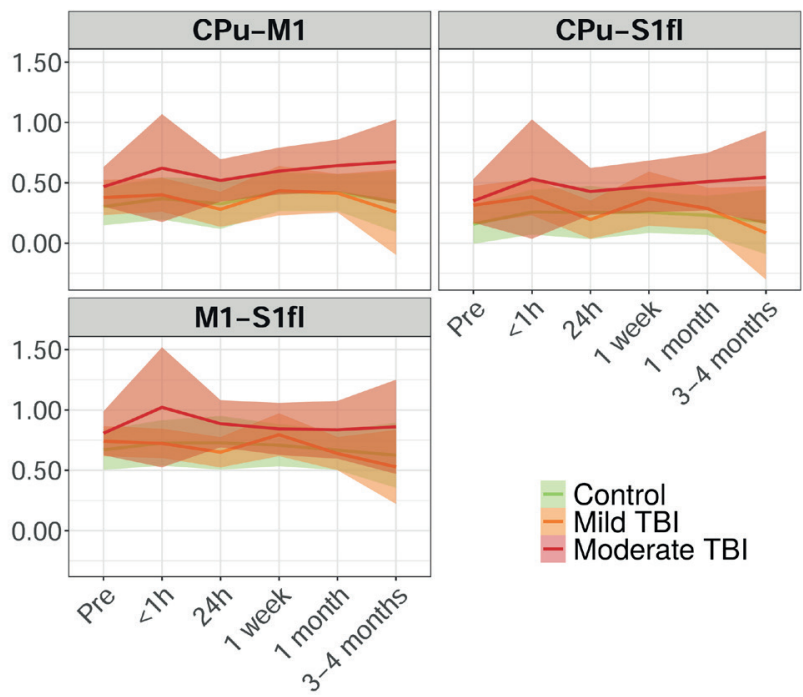

Figure 7. Functional connectivity over time.

Functional connectivity, i.e. Fisher's Z correlation of lowfrequency BOLD fluctuations, calculated between interhemispheric (top) and intrahemispheric (bottom) ROIs, over time for the control (green), mild TBI (orange) and moderate TBI (red) group. Abbreviations: $\mathrm{M} 1=$ primary motor cortex, S1fl = forelimb region of the primary somatosensory cortex, $\mathrm{CPu}=$ caudate putamen. 


\section{Classification of rats based on MRI parameters}

Logistic multiple regression models were used to distinguish between control, mild TBI and moderate TBI based on the acquired MRI parameters. The different models, which contained different parameters, were evaluated using McFadden's pseudo $\mathrm{R}^{2}$ and compared by AIC values and Chi-squared tests.

Diffusion parameters in the hippocampus, corpus callosum and cingulum (i.e. FA, AD and RD; Model 2) significantly attributed to the distinction between mild TBI and control animals 1 hour after TBI but not 24 hours after TBI. At 1 hour after TBI, Model 2 showed a good fit $\left(\mathrm{R}^{2}=0.21\right)$ and significantly improved as directly compared to the null-model $\left(X^{2}=8.5, p<\right.$ 0.05). The fit of Model 1 , which contained $T_{2}$ as additional explanatory variable, did not significantly improve as compared to the null-model as well as compared to model 2 (Table 2). The fits of Models 3, 4 and 5, which all contained less diffusion parameters, significantly deteriorated.

Diffusion parameters and $T_{2}$ (Model 1 ) significantly contributed to the distinction between moderate TBI and control animals at 1 hour after TBI (Table 3), where dropping the $T_{2}$ parameter significantly deteriorated the model (Model 2). At 24 hours after TBI, the $\mathrm{T}_{2}$ parameter did not significantly attribute to the distinction between moderate TBI and control animals, in contrast to the diffusion parameters (Table 4).

\begin{tabular}{lrrrrrrr}
\hline Model & $\boldsymbol{R}^{\mathbf{2}}$ & AIC & $\begin{array}{r}\text { Residual } \\
\text { df }\end{array}$ & $\begin{array}{r}\text { Residual } \\
\text { deviance }\end{array}$ & df & $\boldsymbol{X}^{\mathbf{2}}$ & $\boldsymbol{P}$-value \\
\hline Null & & 43.2 & 31 & 41.2 & & & \\
1. FA + AD + RD + T2 & 0.21 & 41.1 & 27 & 32.4 & 4 & 8.8 & 0.07 \\
2. FA + AD + RD & 0.21 & 40.7 & 28 & 32.7 & -1 & -0.3 & 0.60 \\
3. FA + AD & 0.01 & 46.7 & 29 & 40.7 & -1 & -8.0 & $<0.01$ \\
4. FA + RD & 0.03 & 45.8 & 29 & 39.8 & 0 & 0.9 & - \\
5. AD + RD & 0.02 & 46.4 & 29 & 40.4 & 0 & -0.6 & - \\
\hline
\end{tabular}

Table 2. Results from logistic multiple regression models to differentiate between control and mild TBI rats based on different brain MRI parameters $1 \mathrm{~h}$ post-TBI. Deviance $\left(X^{2}\right)$ is determined by comparing the model with the previous model. Abbreviations: AIC: Aikake Information Criterion; FA: fractional anisotropy; AD: axial diffusivity; RD: radial diffusivity; df: degrees of freedom.

\begin{tabular}{lrrrrrrr}
\hline Model & $\boldsymbol{R}^{\mathbf{2}}$ & AIC & $\begin{array}{r}\text { Residual } \\
\text { df }\end{array}$ & $\begin{array}{r}\text { Residual } \\
\text { deviance }\end{array}$ & df & $\boldsymbol{X}^{\mathbf{2}}$ & $\boldsymbol{P}$-value \\
\hline Null & & 26.1 & 17 & 24.1 & & & \\
1. FA + AD + RD + T2 & 0.79 & 15.1 & 13 & 5.1 & 4 & 19.0 & $<0.01$ \\
2. FA + AD + RD & 0.53 & 19.3 & 14 & 11.3 & -1 & -6.2 & $<0.05$ \\
3. FA + AD & 0.44 & 19.5 & 15 & 13.5 & -1 & -2.2 & 0.14 \\
4. FA + RD & 0.49 & 18.3 & 15 & 12.3 & 0 & 1.2 & - \\
5. AD + RD & 0.48 & 18.6 & 15 & 12.6 & 0 & -0.3 & - \\
\hline
\end{tabular}

Table 3. Results from logistic multiple regression models to differentiate between control and moderate TBI rats based on different brain MRI parameters $1 \mathrm{~h}$ post-TBI. Deviance $\left(\mathrm{X}^{2}\right)$ is determined by comparing the model with the previous model. Abbreviations: AIC: Aikake Information Criterion; FA: fractional anisotropy; AD: axial diffusivity; RD: radial diffusivity; df: degrees of freedom. 


\begin{tabular}{lrrrrrrr}
\hline Model & $\boldsymbol{R}^{\mathbf{2}}$ & AIC & $\begin{array}{r}\text { Residual } \\
\text { df }\end{array}$ & $\begin{array}{r}\text { Residual } \\
\text { deviance }\end{array}$ & df & $\boldsymbol{X}^{\mathbf{2}}$ & $\boldsymbol{P}$-value \\
\hline Null & & 30.3 & 22 & 28.3 & & & \\
1. FA + AD + RD + T2 & 0.50 & 24.1 & 18 & 14.1 & 4 & 14.2 & $<0.01$ \\
2. FA + AD + RD & 0.46 & 23.4 & 19 & 15.4 & -1 & -1.3 & 0.25 \\
3. FA + AD & 0.09 & 31.7 & 20 & 25.7 & -1 & -10.3 & $<0.01$ \\
4. FA + RD & 0.13 & 30.6 & 20 & 24.6 & 0 & 1.1 & - \\
5. AD + RD & 0.10 & 31.4 & 20 & 25.3 & 0 & -0.8 & - \\
\hline
\end{tabular}

Table 4. Results from logistic multiple regression models to differentiate between control and moderate TBI rats based on different brain MRI parameters $24 \mathrm{~h}$ post-TBI. Deviance $\left(\mathrm{X}^{2}\right)$ is determined by comparing the model with the previous model. Abbreviations: AIC: Aikake Information Criterion; FA: fractional anisotropy; AD: axial diffusivity; RD: radial diffusivity; df: degrees of freedom.
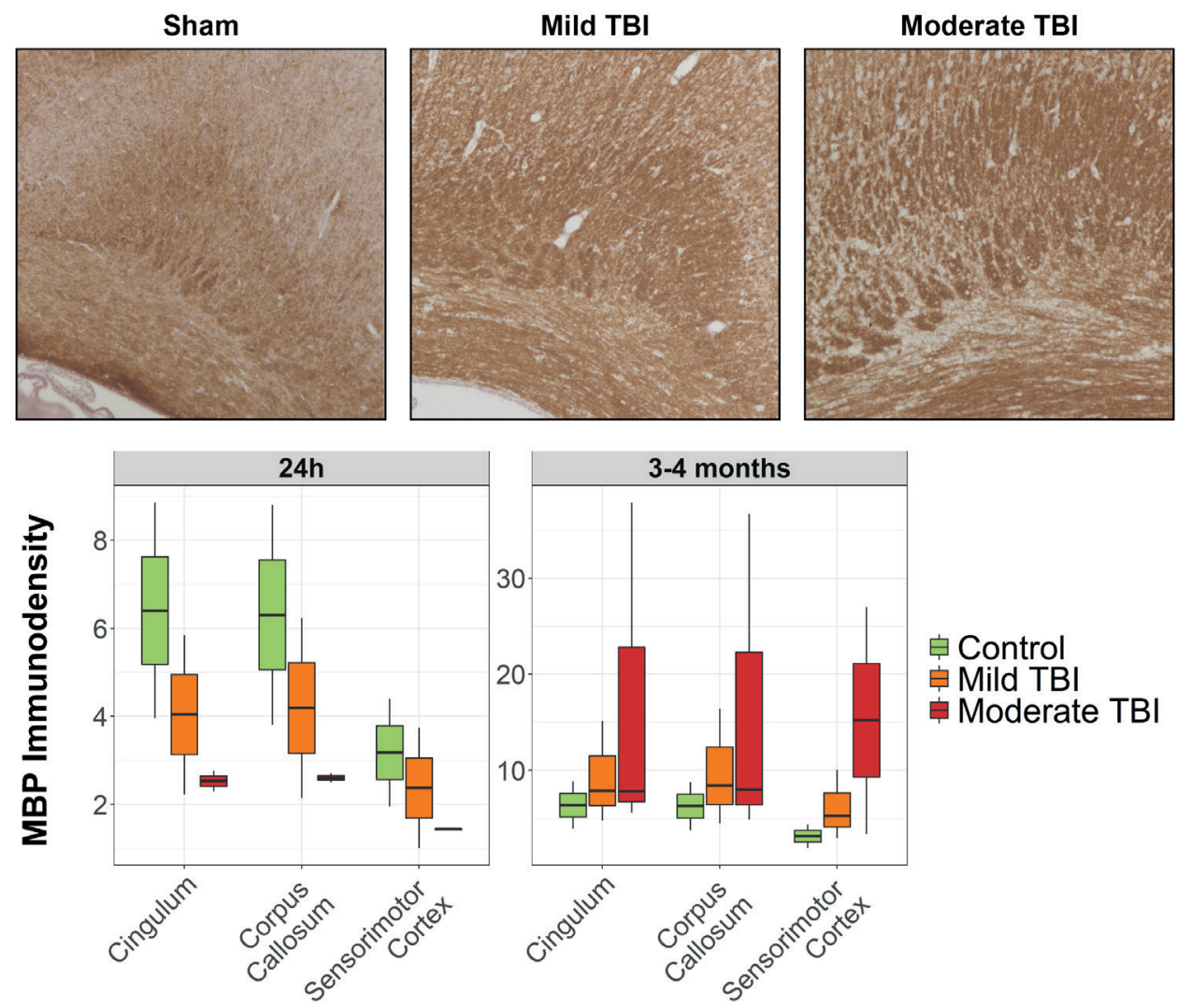

Figure 8. MBP-DAB immunodensity at 24 hours and 3-4 months post-TBI. MBP-DAB staining sections of control, mild TBI and moderate TBI brains 24 hours post-TBI at 40× magnification (top). MBP-DAB immunodensity in the cingulum, corpus callosum and sensorimotor cortex ( $x$-axis) shown at 24 hours (left) and 3-4 months (right) post-TBI for the control (green), mild TBI (orange) and moderate TBI (red) group. Boxplots show median and inter-quartile range (IQR), with whiskers representing 1.5 times the IQR (bottom). 


\section{Immunohistochemistry}

MBP staining was performed to assess differences in myelin density between control, mild TBI and moderate TBI animals at 24 hours and 3-4 months post-TBI (Figure 8). No significant differences were found between control, mild TBI and moderate TBI rats in myelin density in the corpus callosum, cingulum and sensorimotor cortex. Nevertheless, at 24 hours postTBI all regions showed trends of decreasing myelin density from control to mild TBI to moderate TBI. After 3-4 months all regions revealed increased myelin density as compared to 24 hours, which varied considerably in the TBI groups.

\section{DISCUSSION}

In the present study we applied longitudinal multi-parametric MRI to characterize the development of structural and functional changes in grey and white matter regions from acute to chronic stages after mild and moderate TBI. In addition, sensorimotor function was followed over time and post mortem histology was performed to corroborate MRI data. Our data show that moderate TBI induces early changes in white matter regions, which dissipate over time, in parallel with loss and recovery of sensorimotor function. Correspondingly, histology revealed reduced myelin density in the acute stages after TBI, which was not detected in the chronic stages. Although no significant changes in MRI markers were found after mild TBI, our multivariate model demonstrated the added value of combining diffusion parameters in the distinction between mild TBI and control brain.

Earlier studies have used diffusion MRI to characterize white matter changes following brain trauma in experimental models and patients (Bazarian et al., 2007; Edlow et al., 2016; Inglese et al., 2005; Ling et al., 2012; van de Looij et al., 2012; Mayer et al., 2010; Palacios

et al., 2012; Rutgers et al., 2008; Tu et al., 2016; Wang et al., 2018). In the present study we used the Marmarou weight-drop (acceleration impact) model (Foda and Marmarou, 1994; Marmarou et al., 1994), which has been shown to mimic the widespread axonal damage of human TBI (Wang and Ma, 2010). Our findings generally agree with previous studies using TBI model, in which significant changes in white matter structures and the hippocampus were measured early after moderate/severe TBI induction (van de Looij et al., 2012; Tu et al., 2016), but not after mild TBI induction (Singh et al., 2016). However, other studies have reported damage in white matter tracts (e.g. cingulum, hippocampal white matter, corpus callosum and pyramidal tract) from days up to one week after mild TBI (Braeckman et al., 2019; Li et al., 2013). Histology revealed axonal damage in the corpus callosum, internal capsule and pyramidal tract acutely after mild TBI (Li et al., 2013). Furthermore, fiber damage has been detected in the corpus callosum from acute to sub-acute stages after moderate/severe TBI (Braeckman et al., 2019; van de Looij et al., 2012; Tu et al., 2016), which normalized chronically after TBI (Tu et al., 2016). These findings correspond with the patterns of MBP immunodensity values in our study, which reflected reduced myelin 
density in the corpus callosum, cingulum and cortex acutely after mild and moderate TBI, which was restored and increased several months later. The absence of clear diffusion changes in response to microscopic histological alterations may suggest that contemporary diffusion MRI techniques lack sensitivity for detection of subtle white matter alterations. However, our multivariate logistic regression model showed that combined assessment of different diffusion parameters can lead to improved differentiation between unaffected and mild or moderate TBI-affected brains acutely after TBI.

Early after moderate TBI, we found increased radial and axial diffusivity in the corpus callosum, hippocampus and cingulum, and decreased FA tended in the corpus callosum and hippocampus. These changes in tissue water characteristics may be related to structural white matter injury, such as axonal damage and demyelination (Song et al., 2005; Sun et al., 2006; Tuor et al., 2014; Winklewski, 2018). On the other hand, the early occurrence of these diffusion changes and their transient nature (normalization within one week), are more suggestive of dynamic water shifts, such as formation and subsequent resolution of white matter edema. This assumption is corroborated by the similar pattern in $T_{2}$ changes and the transient changes in myelin density.

Significant diffusion changes were not found in white matter, such as the internal capsule, which was more distant to the impact site. However, FA in the cingulum and internal capsule of moderate TBI animals did not show a characteristic increase over time as found in control animals. Previous diffusion tensor imaging studies reported increasing FA in white matter structures of rats during adulthood up to old age, which has been related to ongoing white matter maturation (Blockx et al., 2011; Mengler et al., 2014). This is also in agreement with our findings of higher myelin densities in corpus callosum and cingulum after 3-4 months, although there was quite some (unexplained) variation in the moderate TBI group. Thus, lack of gradual FA increase in the internal capsule after moderate TBI, may be reflective of disturbed white matter maturation.

Neurological deficits paralleled the temporal patterns of the white matter alterations. Loss of sensorimotor function was apparent early after moderate TBI, but not after mild TBI or in control animals. In line with our brain MRI findings, sensorimotor function normalized one week after moderate TBI. Some studies have applied diffusion MRI to characterize postTBI white matter changes in relation to behavioral deficits, and reported correlations between white matter FA and neurological deficits (Long et al., 2015; Wang et al., 2017). However, these studies used a controlled cortical impact model, inducing more focal and lateralized brain damage, in contrast to our acceleration-deceleration model. Heffernan et al. (2013), who used a weight-drop model, found significant differences between mild TBI and control animals in cognitive tasks, but no differences in performance on the beam walk test (Heffernan et al., 2013). Unfortunately, we have no cognitive performance data in our study, but the beam walk test results are in line with our findings in mild TBI animals. 
Despite our findings of altered $\mathrm{T}_{2}$ and diffusion parameters, we observed no significant BBB leakage in mild or moderate TBI. In two studies that used a similar weight-drop acceleration impact model, brief opening of the BBB was measured (Barzó et al., 1996; Beaumont et al., 2000), which was restored 30 minutes after TBI (Barzó et al., 1996). Our first MRI measurement of BBB integrity was 1-2 hours after TBI induction, which may have been too late to detect such a brief and subtle opening.

Our analyses of functional connectivity from rs-fMRI scans revealed overall higher interand intra-hemispheric functional connectivity in moderate TBI rats, compared to mild TBI and control rats. This seems in contrast with previous studies that reported decreased functional connectivity after TBI, observed in rats (Heffernan et al., 2013) and human patients (Arenivas et al., 2014; Castellanos et al., 2010; Mayer et al., 2011; Palacios et al., 2013). Heffernan et al., (2013) found decreased functional connectivity in a specific connection between the infralimbic cortex and anterior cingulate cortex 24 hours after TBI in rats, whereas no functional connectivity alterations were found in the somatosensory cortex (Heffernan et al., 2013). Decreased functional connectivity between specific regions has also been reported in human TBI patients (Arenivas et al., 2014; Mayer et al., 2011). Yet, other studies found increased global functional connectivity after TBI-induced white matter damage (Hillary et al., 2011; Palacios et al., 2013; Sharp et al., 2011, 2014). Discrepancies among studies may be explained by the heterogeneity in TBI pathology as well as differences in the investigated connections and functional networks. Subtle and widespread white matter damage may induce compensatory changes (e.g. increase) in functional connectivity, which has been observed in multiple sclerosis (Hawellek et al., 2011), whereas more severe and focal white (or grey) matter damage could lead to loss of functional connectivity, which has been observed after stroke (van Meer et al., 2010b, 2012). Accordingly, cognitive improvement after TBI has been related to an increase in functional connectivity, reflective of adaptive or compensatory mechanisms (Hawellek et al., 2011; Palacios et al., 2013; Sharp et al., 2011). Clearly, more studies are needed to elucidate the complex relationship between structural brain damage, functional connectivity changes, and behavioral performance.

Overall our findings suggest that diffusion MRI may contribute to the diagnosis and classification of TBI. Nowadays, TBI is primarily diagnosed on patients' self-report of clinical symptoms, sometimes complemented with conventional imaging modalities like CT and MRI (Borg et al., 2004; Maas et al., 2008). However, a large majority of mild TBI patients has normal MRI scans (Belanger et al., 2007; Borg et al., 2004), so more sensitive techniques are required to detect subtle alterations in the brain. Our logistic modeling showed that diffusion parameters (i.e. FA, $A D$ and $R D$ ) early after $T B I$ have additional value in distinguishing mild TBI. Moreover, more sophisticated diffusion MRI techniques, such as diffusion kurtosis imaging, apparent fiber density analyses and fixel-based analysis, may 
lead to better characterization of diffusion metrics in white matter, enabling more sensitive and specific identification of white matter pathology (Braeckman et al., 2019; Verhelst et al., 2019; Wright et al., 2017). Future studies should explore how advancements in diffusion $\mathrm{MRI}$, combined with other structural and functional MRI measures, could further improve the diagnosis of patients with mild TBI symptoms. 



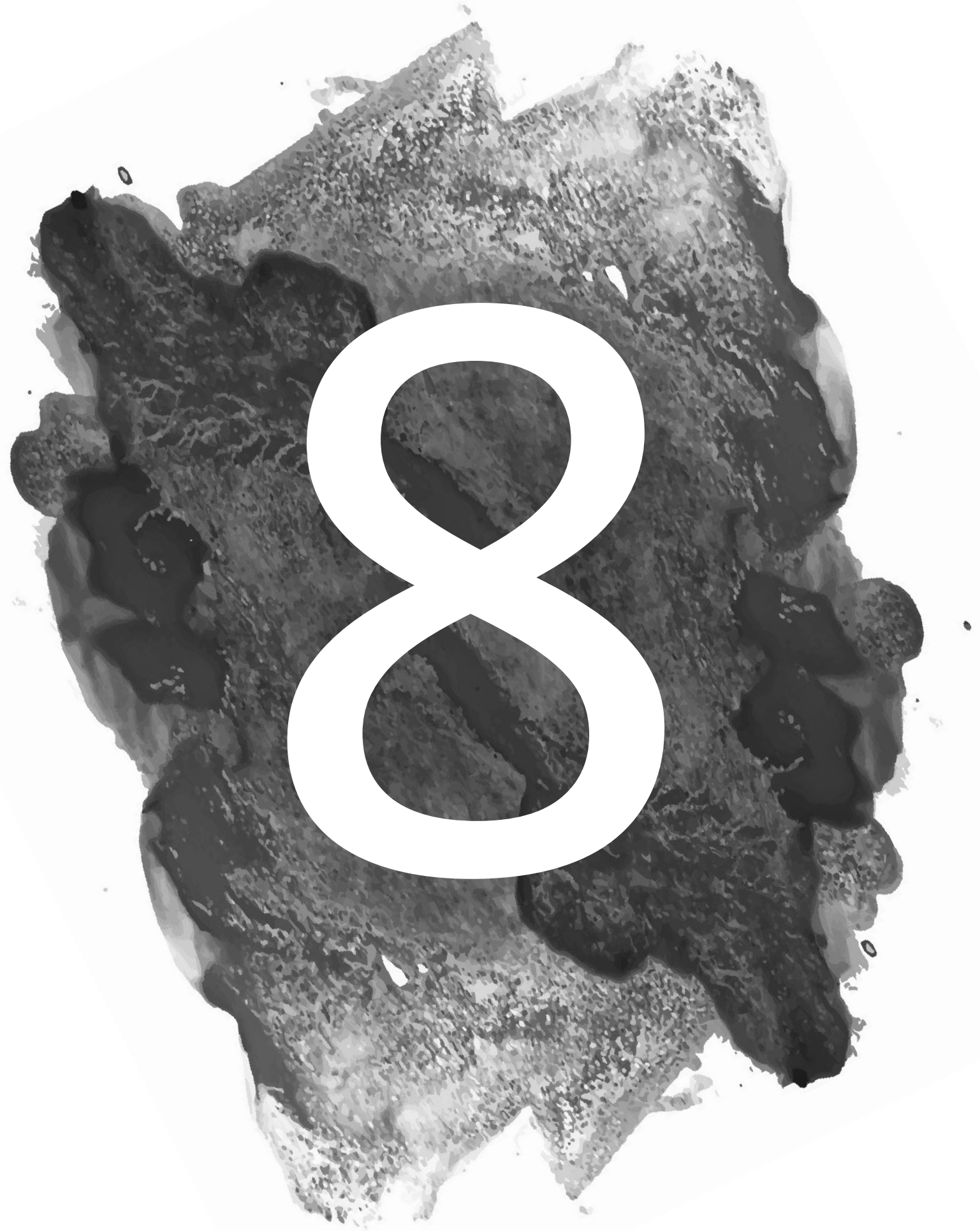




\section{Chapter 8}

\section{General Discussion}





\section{GENERAL DISCUSSION}

The main objective of the present thesis was two-fold. First, we aimed to characterize the spatiotemporal patterns of neuronal reorganization after brain damage induced by stroke or traumatic brain injury (TBI) from a brain network perspective. To that aim, we used structural and functional brain MRI data from rat stroke and TBI models. More specifically, we used diffusion MRI-based tractography to map macro-scale structural connectivity, whereas resting-state functional MRI was used to measure functional connectivity. Furthermore, we analysed alterations in specific structural and functional connections, as well as in (the topology of) whole-brain structural and functional networks by using different network analysis approaches. Second, we aimed to elucidate the effect of ageing on brain network vulnerability to lesions and damage. To that aim, we used brain MRI data from human and rat lifespan datasets. We simulated lesions at different stages across the lifespan to characterize the consequences of these lesions for the structural and functional network topology.

The findings in this thesis demonstrate that (I) advanced in vivo MRI techniques provide unique tools to longitudinally investigate structural and functional neural networks after stroke (Chapters 2, 5 and 6) and TBI (Chapter 7), in relation to changes in behavior (Chapters 4, 6 and 7); (II) at connectome level more advanced tractography algorithms, such as constrained spherical deconvolution (CSD) and global tractography, do not perform better than conventional DTI-based tractography, and many factors affect the final connectome reconstruction resulting in an overall trade-off between reconstruction sensitivity and specificity (Chapter 3); (III) stroke induces remote alterations in specific tracts (e.g. the corticospinal tract) (Chapter 6), and specific structural and functional networks, which include the contralesional hemisphere (Chapters 4 and 5); (IV) structural and functional networks seem to be more vulnerable to lesion damage in aged people and rats (Chapters 2 and 5); (V) recently developed network analysis methods, such as Bayesian Exponential Random Graph models (Chapter 2) and Minimum Spanning Tree (MST) analysis (Chapter 4), are applicable to rat and human brain networks and may reveal new or complementary insights in addition to conventional network analysis methods.

This chapter will summarize and discuss the most important findings from the studies in this thesis. Furthermore, we will discuss pitfalls of the used MRI techniques and network methodologies as well as potential directions with regard to future studies on brain plasticity.

\section{The brain as a complex and integrative network}

As discussed in the Introduction of this thesis, network approaches are gaining importance for the assessment of injury and recovery mechanisms in brain disorders. 
In this thesis we therefore used a network perspective to focus on changes in critical pathways, such as the transcallosal and corticospinal tracts (chapters 6 and 7), as well as whole-brain networks (chapters 2, 4 and 5). Post-stroke neuronal network reorganization has been shown to occur from micro-scale (i.e. synaptic plasticity, axonal sprouting) to macro-scale (i.e. cortical remapping) (Biernaskie and Corbett, 2001; Jones et al., 1996; Stroemer et al., 1995). Furthermore, neuronal network reorganization extends from perilesional sites to more distant (i.e. contra-lesional) sites (Cai et al., 2016; Crofts et al., 2011; Dacosta-Aguayo et al., 2014; Granziera et al., 2012; Gratton et al., 2013; JohansenBerg et al., 2010; Schaechter et al., 2010). After brain trauma, widespread axonal damage, cell death and ongoing neurodegeneration have been observed (Andriessen et al., 2010; Bains and Hall, 2012; Masel and DeWitt, 2010; Xiong et al., 2013). Accordingly, we measured structural and functional network alterations at distant sites from the stroke or TBI lesion in our studies.

Signs of remote white matter degeneration were detectable in the corticospinal tract after focal cortical stroke, which increased between 1 and 28 weeks post-stroke (chapter 6). Corticospinal tract damage has previously been found after stroke in humans and rats, in relation to poorer functional outcome (Lindberg et al., 2007; Lindenberg et al., 2010, 2013; van Meer et al., 2012; Schaechter et al., 2010; Tuor et al., 2014). Despite lasting corticospinal tract degeneration, however, our study showed that interhemispheric functional connectivity can partially restore chronically after stroke. The changes in interhemispheric functional connectivity had a similar temporal pattern as the changes in sensorimotor performance (chapter 6). Hence, our results indicate that functional recovery may be more dependent on recovery of interhemispheric functional connectivity, supported by intact transcallosal tracts (van Meer et al., 2010a, 2010b).

Signs of white matter tract damage, specifically in the corpus callosum, cingulum, hippocampus and internal capsule, were also found after moderate TBI (chapter 7). The temporal patterns of which were similar to temporal patterns in neurological deficits. Correspondingly, histology revealed decreased myelin staining early after TBI, which was not detected at chronic stages. In contrast to our findings in the stroke model, overall interhemispheric functional connectivity was increased after moderate TBI (chapter 7). Other TBI studies have also reported increased global functional connectivity along with white matter damage (Hillary et al., 2011; Palacios et al., 2013; Sharp et al., 2011, 2014). It has been suggested that increased global functional connectivity is indicative of compensatory mechanisms and related to reduced cognitive impairment (Hawellek et al., 2011; Palacios et al., 2013; Sharp et al., 2011). The discrepancy between stroke- and TBIinduced changes in interhemispheric functional connectivity probably relates to the nature (i.e. focal damage versus more widespread and global damage) or the extent of the induced brain damage. 
The studies in this thesis also revealed contra-lesional structural network alterations chronically after stroke (chapter 4 ) as well as global structural and functional network changes after lesion simulations in rat (chapter 5 ) and human brains (chapter 2). Shifts in hub-regions were apparent in structural networks (chapters 4 and 5) and functional networks (chapter 5), in the ipsi- and contralesional hemispheres. Changes in neural network organization in the contralesional hemisphere after stroke may reflect remote tissue degeneration as well as remote tissue plasticity (Biernaskie and Corbett, 2001; Carter et al., 2012a; Crofts et al., 2011; Dijkhuizen et al., 2012; Grefkes and Fink, 2011; Jones et al., 1996; Stroemer et al., 1995). We found increased hubness and structural connectivity strength in contralesional regions (e.g. upper lip and jaw regions of the primary somatosensory cortex) chronically after stroke, which suggest adaptive reorganization of the contralesional sensorimotor network, possibly through strengthening of structural connections (chapter 4). This adaptive contra-lesional reorganization corresponds with findings of enhanced in vivo inter-hemispheric functional connectivity in previous publications from our group (van Meer et al., 2010a, 2010b). We also found shifts in hub regions in structural and functional networks after simulating different type of stroke lesions, which depended on the size and location of the stroke injury (chapter 5). Together these findings again emphasize the key role of hub regions in (the outcome of) brain disorders, such as stroke and TBI (Aerts et al., 2016; Alstott et al., 2009; Crossley et al., 2014; Stam, 2014).

In addition to nodal changes (i.e. strength, hubness) across the brain, lesions also affected global network topology at whole-brain level (chapters 2 and 5) or in the contralesional hemisphere as a whole (chapter 4). To the best of our knowledge there have been no previous studies on macro-scale structural networks after experimental stroke in rats (chapter 4). Our findings of decreased global efficiency of structural networks in the contralesional hemisphere are in line with human studies that reported contralesional structural network modifications (Cheng et al., 2019a; Crofts et al., 2011). Global network efficiency in the contralesional hemisphere was related to sensorimotor outcome (chapter 4), which may point toward the importance of efficient contralesional network functioning for recovery in stroke patients. We also found decreased global efficiency and increased clustering or segregation in whole-brain structural and functional networks after lesion simulations (chapter 5), which fit previous research (Carter et al., 2012a; Cheng et al., 2019a; Crofts et al., 2011; Grefkes and Fink, 2014; van Meer et al., 2012). The effects depended on the extent of the lesion, which is also largely in agreement with our previous work, where effects were also considerably smaller when lesions were limited to only subcortical or cortical tissue (van Meer et al., 2012). In our simulation study, lesions in human hub-regions had a larger effect on the network topology (chapter 2), which has been shown previously (Alstott et al., 2009; Crossley et al., 2014; Kaiser et al., 2007). Together 
these findings further substantiate that focal brain damage can lead to significant deviation from an optimal and efficient brain network topology (i.e. small-worldness) (Bassett and Bullmore, 2009; Bullmore and Sporns, 2009; van Meer et al., 2012; Sporns, 2010; Stam, 2014). Studies on post-stroke modification in whole-brain structural networks are scarce in comparison to studies on whole-brain functional networks (Aerts et al., 2016; Carter et al., 2012a; Cheng et al., 2019a; Crofts et al., 2011; Grefkes and Fink, 2011). Future studies should therefore further elucidate how whole-brain structural networks are affected by brain lesions and how this relates to functional connectivity and behavioural function. Animal models provide unique possibilities to perform longitudinal, multiparametric and experimental assessment of whole-brain structural and functional reorganization after stroke or TBI (Dijkhuizen and Nicolay, 2003; Fluri et al., 2015; Xiong et al., 2013). In addition, these studies may be strengthened by the application of recently developed neurostimulatory techniques such as transcranial magnetic stimulation (TMS) (Lindenberg et al., 2010; Stinear et al., 2007; Villamar et al., 2012) and Designer Receptors Exclusively Activated by Designer Drugs (DREADDs) (Roelofs et al., 2017; Tu and Zhang, 2019) in rodents. Both DREADDs and TMS have already been successfully applied in our lab (Boonzaier et al., 2018; Roelofs et al., 2017). These neurostimulatory techniques provide unique means to activate and manipulate specific regions or pathways in vivo, which allows more direct assessment of the functional integrity of neural pathways as well as the influence of brain activity on plasticity mechanisms. Furthermore, application of these techniques in pre-clinical experimental settings may reveal new therapeutic targets and strategies to stimulate brain plasticity and functional recovery after stroke or TBI.

\section{Ageing-related brain network organization and vulnerability}

In this thesis we also investigated the effect of ageing on structural and functional networks with or without lesions (chapters 2 and 5). Healthy structural and functional networks of both young adult and aged rats showed similar global network characteristics, quantified as small-worldness, global efficiency and local clustering (chapter 5). These topological characteristics have been observed previously in human studies as well (Gong et al., 2009; Zhu et al., 2012). We also showed stable contribution of generative network building blocks (i.e. clustering, global efficiency) to a healthy global structural network topology across the human lifespan (chapter 2). Some previous studies found an ageing effect in global efficiency (Otte et al., 2015; Wu et al., 2012; Zhu et al., 2012), whereas other studies do not report differences in global efficiency in structural networks across age (Gong et al., 2009). These outcome differences may be explained by the use of different age categories and methodologies across studies. Furthermore, previous studies showed an inverted U-curve in global efficiency across the lifespan (Otte et al., 2015; Wu et al., 2012; Zhao et al., 2015). This inverted U-curve in global efficiency may also be applicable to the rat brain. However, 
since we only characterized network topology of young-adult and aged rats (which may be on both extremities of the curve), we may have missed this longitudinal ageing effect on the global structural network topology (chapter 5).

Although we did not find a statistically significant ageing effect on global functional networks, we did see a trend towards decreased global efficiency and small-worldness (chapter 5), resembling previous described effects of ageing in humans (Damoiseaux, 2017; Sala-Llonch et al., 2014). In contrast to global network characteristics, we identified clear local differences in structural and functional brain networks between young-adult and aged rats. Structural connectivity strength increased with age for many regions (such as the caudate putamen and bilateral primary motor cortices). We also found increased homotopical symmetry in hub regions in aged rats, which has also been reported for ageing humans (Agcaoglu et al., 2015). This increased homotopical symmetry may also affect the extent of stroke-induced damage and related behavioural dysfunction as well as subsequent recovery in aged rats (van Meer et al., 2010b, 2012).

Lesion simulations were performed to examine the effect of ageing on the vulnerability of structural (chapters 2 and $\mathbf{5}$ ) and functional (chapter 5) networks to different degrees of random and targeted brain damage. Lesion-induced hub shifts were different between young-adult and aged rats (chapter 5). Also in aged people hub-lesions had a larger effect on structural network topology as compared to younger people (chapter 2). It is important to realize that our computational studies only examined direct effects of simulated lesions on the network topology by elimination of one (i.e. a small focal lesion) or multiple nodes (i.e. a larger regional lesion). This approach does not account for dynamic network responses, like subsequent degeneration, regeneration or delayed neurovascular response, which can strongly depend on age (Betzel et al., 2014; Burke and Barnes, 2006; Damoiseaux, 2017; Niccoli and Partridge, 2012; Wang et al., 2015). Nevertheless, computational lesion studies may substitute, complement or guide future empirical studies in human patients or animal disease models (Aerts et al., 2016; Alstott et al., 2009; Crossley et al., 2014; Kaiser et al., 2007).

Taken together, our findings indicate that lesion-induced reorganization in structural and functional networks differ between young-adult and aged rats (chapter 5) and humans (chapter 2). These age-related differences in network topology may in turn lead to increased vulnerability to network damage as well as to different plasticity mechanisms with ageing. This, for instance, could impact experimental stroke studies that are generally performed in young adult rats, although we know that the risk and prevalence of human stroke is higher in older people. This may contribute to the poor bench-to-bedside translation from animal stroke models to human stroke patients (Dirnagl, 2016). Since age is an important risk factor for stroke that also affects the outcome and response to therapies in experimental models (Herson and Traystman, 2014; Liang et al., 2016; Liu et al., 2009), future pre-clinical studies 
should systematically and experimentally study the effects of ageing on structural and functional brain network plasticity in relation to damage and recovery.

\section{Diffusion MRI-based structural integrity}

We used diffusion MRI to assess structural integrity of white matter tracts (chapters 6 and 7), and diffusion-based tractography to map structural connections between regions (chapter 6) and at whole-brain level (chapters 2-6).

After experimental stroke (chapter 6) and TBI (chapter 7) we found alterations in diffusivity parameters. We measured lower FA and higher RD values in the ipsilesional CST outside (i.e. remote from) the focal (cortical) lesion area in stroke rats (chapter 6). These signs of remote white matter damage may be explained by anterograde, retrograde and/or transneuronal degeneration (Cheng et al., 2019a, 2019b; Duering et al., 2015). Also early after moderate TBI we found (a tendency towards) decreased FA together with increased $A D$ and $R D$ in the hippocampus, corpus callosum and cingulum (chapter 7). These early abnormalities in diffusion metrics normalized after one week, suggestive of early formation and subsequent resolution of white matter oedema. In addition there structural axonal damage might contribute to these alterations in tissue water diffusivity, as shown before in TBI studies (Braeckman et al., 2019; van de Looij et al., 2012; Tu et al., 2016), which was confirmed by our histological findings (chapter 7 ). We measured increased AD up to one week after moderate TBI (chapter 7). Although axonal damage has been reported to result in decreased AD (Kumar et al., 2009; Li et al., 2011; Winklewski, 2018), axonal degeneration may also lead to increased extra-axonal space, which in turn could increase water diffusion along axonal fiber bundles (Rosas et al., 2010).

Although the diffusion tensor model is still one of the most frequently used models, it nonetheless oversimplifies the complex tissue structure in the brain. The tensor model is insufficient to capture the complex white matter structures, such as crossing, bending or fanning fibers at the sub-voxel level (Jbabdi and Johansen-Berg, 2011; Tournier et al., 2011). These complex structures are present at multiple sites in the brain (Jeurissen et al., 2013). Furthermore, the tensor model assumes a Gaussian distribution of diffusion, whereas the complex tissue of the brain restricts water diffusion and causes deviation from a Gaussian diffusion displacement distribution (Jensen et al., 2005). Therefore, novel and more complex models have been developed such as multi-tensor models (Chu et al., 2015), diffusion kurtosis imaging (Jensen et al., 2005) (i.e. to measure deviation from the Gaussiandistributed signal), compartment models like CHARMED (Assaf and Basser, 2005) and NODDI (Zhang et al., 2012) (i.e. to assess microstructural integrity by alternative measures such as axonal and neurite density), constrained-spherical deconvolution (CSD) (Tournier et al., 2004) (i.e. to allow for modelling of multiple fiber orientations) and fixel-based analysis (Raffelt et al., 2017) (i.e. to assess characteristics of different fiber segments at sub-voxel 
level). Despite all these developments and improvements, diffusion models are still not fully capturing the measured diffusion signals and are limited with regard to the high structural complexity in the brain (Alexander et al., 2017). Therefore, it is important to cautiously interpret findings from diffusion MRI, particularly in diseased brain. Altered diffusion behaviour in lesioned tissue may involve different mechanisms related to white matter damage, such as increased extracellular fluid, changes in axonal water content or alterations in water flux due to tissue breakdown (Alexander et al., 2017; Beaulieu, 2002). Furthermore, histological examination can complement (or validate) diffusion MRI findings, which is possible in animal models. Importantly, diffusion-based MRI metrics that are more specific to (pathological) characteristics of the underlying tissue structure (Alexander et al., 2017; Assaf et al., 2017; Beaulieu, 2002; Winklewski, 2018), may lead to further improvement of the diagnostic potential of diffusion MRI.

\section{Diffusion-based tractography and structural connectivity}

Diffusion MRI can also be used to map white matter tracts using a technique called fiber tractography (or tracking) (Basser et al., 2000; Jbabdi and Johansen-Berg, 2011; Jbabdi et al., 2015; Jeurissen et al., 2017). Diffusion-based tractography was used to reconstruct specific white matter tracts such as the CST and transcallosal tracts (chapter 6 ) as well as large-scale structural connectivity in the brain (chapters 2-6). Furthermore, we validated whole-brain structural connectome reconstructions against neuroanatomical connectivity from tracer studies (chapter 3).

We compared and evaluated high-resolution diffusion MRI-based whole-brain structural connectome reconstructions, generated by DT-based, CSD-based and global tractography, against neuroanatomical tracers (chapter 3 ). All algorithms were able to reconstruct a large variety of brain-wide structural connections, although considerable amounts of false positive and false negative connections were detected as well, in line with earlier studies (Azadbakht et al., 2015; Delettre et al., 2019; Donahue et al., 2016; Maier-Hein, 2017; Schilling et al., 2018; Thomas et al., 2014). We found that specific parameter settings (e.g. FA threshold for DT-based tractography, number of generated streamlines or the particle potential for global tractography) influenced the connectome reconstruction sensitivity and specificity. Furthermore, the application of SIFT, streamline thresholds and group-based average network thresholding, also affected the sensitivity and specificity. Overall, improvements in sensitivity due to adjustments of algorithm, parameter settings or subsequent tractogram thresholding or filtering, were invariably at the expense of specificity (chapter 3). As such, our findings agree with reported trade-offs between sensitivity and specificity of reconstruction of tracts or whole-brain connectomes (Bastiani et al., 2012; Chen et al., 2015; Delettre et al., 2019; Donahue et al., 2016; Maier-Hein, 2017; Sarwar et al., 2019; Schilling et al., 2019; Thomas et al., 2014). 
Fiber tractography involves a multi-stage process including many parameters. Different parameters lead to different results, irrespective of the raw measures. Parameter choices are related to the applied diffusion model (e.g. tensor, CSD), fitting approach (e.g. local versus global, deterministic versus probabilistic), fiber selection and termination criteria (e.g. FA threshold, angle threshold) and the fiber seeding mechanism (e.g. global, dynamic) (Jeurissen et al., 2017) (chapter 3). This is a problem for comparison of results across studies using different algorithms and methodologies. Future developments of tractography algorithms should focus on improved reconstruction of existing tracts (i.e. increased sensitivity) without the introduction of more false positives (i.e. stable or even increased specificity). The inclusion of anatomical constraints (Lemkaddem et al., 2014; Smith et al., 2012) and priors (Christiaens et al., 2014, 2015b; Yendiki et al., 2011) or microstructurally informed tractography (Daducci et al., 2016; Dell'Acqua et al., 2013; Girard et al., 2017; Schiavi et al., 2019), may guide and improve tractography-based reconstructions of tracts and whole-brain networks. Also machine learning-based approaches have shown potential in improving the specificity of tractography-based connectome reconstructions (Neher et al., 2017). Improvement in reconstruction accuracy will also affect (and improve) graphanalytical studies on the structural connectome (Bullmore and Sporns, 2009; Zalesky et al., 2016).

Diffusion-based tractography has previously been applied in stroke patients to map specific white matter tracts (Chia-Koh et al., 2018; Lindenberg et al., 2010, 2013) and has also been successfully applied in rats (Figini et al., 2015). We used this approach to map, segment and analyse the bilateral CST and transcallosal tracts in a rat stroke model (chapter 6). We determined diffusivity characteristics across these streamlines, which could yield more specific information as compared to region-based analysis. Furthermore, we used different diffusion-based tractography approaches to reconstruct whole-brain structural networks in humans across the lifespan (chapter 2), in young-adult and aged rats (chapter 5), as well as chronically after stroke using post-mortem brain samples (chapter 4). We were able to detect structural network changes across the lifespan of rats (chapter 5) and after stroke (chapter 4) or simulated damage (chapters 2 and 5).

Despite their limitations, diffusion MRI and subsequent fiber tractography are the only non-invasive tools to map structural brain connectivity in vivo. Recent work shows that whole-brain connectomes are highly reproducible when using sufficient number of streamlines and appropriate thresholding of structural connections (Roine et al., 2019). Hence, diffusion MRI-based tractography will continue to provide unique possibilities to map structural connectivity longitudinally in clinical and experimental studies. 


\section{Functional MRI-based resting-state functional connectivity}

We used resting-state $\mathrm{fMRI}$ to measure functional connectivity for specific regions between and within hemispheres (chapters 6 and 7), as well as to map whole-brain functional networks (chapter 5). The classical resting-state functional connectivity measurement calculates the temporal correlation (often the Pearson's correlation coefficient) of spontaneous low-frequency fluctuations between different regions, whereby the magnitude of the correlation indicates the strength of the functional connection. In this thesis we used this approach to map whole-brain functional networks in young-adult and aged rats and to determine local and global topological characteristics (chapter 5). Furthermore, we determined interhemispheric functional connectivity between homotopic regions (chapters 6 and 7), and intrahemispheric functional connectivity between cortical and sub-cortical regions (chapter 7). In our studies we only considered positive functional connectivity values, since there is no consensus about the nature and interpretation of negative-valued functional connectivities (Murphy and Fox, 2017; Murphy et al., 2009).

The classical resting-state $\mathrm{fMRI}$-based functional connectivity measurement is robust and straightforward to apply, without generating enormous amounts of data and the need of complex statistical analyses and modelling approaches. These are advantages for implementation in a clinical setting. However, the classical approach of functional connectivity measurement does not account for the dynamic and complex functional interactions in the brain. Therefore, novel approaches have been developed to capture the dynamic nature of functional brain networks, such as the sliding window approach (Hutchison et al., 2013; Preti et al., 2017; Shen et al., 2012) or modelling of dynamic and effective connectivity (Avena-Koenigsberger et al., 2017). These novel approaches could shed new light on functional plasticity mechanisms in the brain. For example, effective connectivity analyses may reveal rerouting pathways in the brain after brain damage. This may elucidate whether post-stroke interhemispheric connectivity normalization, after initial decrease, is explained by restoration of direct or indirect functional connectivity pathways (or both). Or, from a dynamic functional connectivity perspective, there might still be significant abnormalities in functional network dynamics after stroke, even when conventional functional connectivity measures show normalization. Applying these complex dynamic functional connectivity analyses in a research setting may reveal potential therapeutic targets (i.e. specific pathways that should be stimulated at specific timepoints). In addition to more advanced functional network modelling approaches, resting-state fMRI could be combined with other functional imaging techniques, such as optical imaging techniques (Bauer et al., 2017; Bergonzi et al., 2015; White et al., 2011) and electroencephalography (van Diessen et al., 2015), which may yield additional and complementary information on functional network dynamics. 
The relationship between structural and functional connectivity in the brain was not specifically addressed in this thesis. However, previous research has shown a clear relationship between different levels of structural connectivity (i.e. from neuronal tracer and diffusion-based tractography studies) and functional connectivity (Straathof et al., 2020), which we have recently described in a review (Straathof et al., 2019). How structural and functional connectivity exactly relate across different levels of investigation (i.e. from micro- to macro-scale) still remains unknown. Neither has this been explored under pathological conditions. For example, to explain how the restoration of interhemispheric functional connectivity after stroke depends on underlying neuronal changes (e.g. formation of synapses, axonal sprouting etc.). Or how changes in functional connectivity induced by neurostimulation, for example with transcranial magnetic stimulation (Fox et al., 2012), relate to (modifications in) the underlying neuronal substrate. Studies already described disturbed structure-function relationships in different brain disorders, such as idiopathic generalized epilepsy (Zhang et al., 2011) and ischemic stroke (Zhang et al., 2017). Therefore, future studies may employ specific measures of the relationship between structural and functional connectivity to further investigate brain damage-induced network reorganization and how this relates to behavioural function.

\section{Brain network analysis methods}

Structural and functional brain networks can be characterized and analysed with multiple network analysis methods. Different network analysis approaches reveal different aspects of brain network topology. Descriptive network approaches aim to characterize topological characteristics of brain networks at local and global level, whereas generative network approaches aim to identify generative rules that guide brain network development and emergent topological characteristics. In this thesis we applied classical graph analysis (chapter 5), Bayesian exponential random graph models (ERGM) (chapter 2) and minimum spanning tree (MST) analysis (chapter 4).

Classical graph analysis is a descriptive network approach, which allows the characterization of the global network topology as well as descriptive nodal characteristics. Despite the usefulness of classical graph analysis to characterize the global network topology, there are intrinsic limitations. Frequently applied network metrics, such as the clustering coefficient and shortest path length, are affected by the number of nodes and edges in the network (Fornito et al., 2013; van Wijk et al., 2010). To minimize these effects we normalized our metrics using random generated networks (chapter 5). Still, these normalized metrics may be affected by the number of nodes and edges (van Wijk et al., 2010). Furthermore, since networks are highly interdependent by nature, many network metrics are strongly correlated and non-exclusive (Bounova and de Weck, 2012; Meghanathan, 2015; Roine et al., 2019). Recently, it has therefore been suggested to select 
a limited number of metrics to improve statistical power (Roine et al., 2019). Nevertheless, classical graph analysis can reveal critical changes in brain network topology. For example, we were able to detect global network topology alterations after simulations of a large stroke (chapter 5). Besides, nodal characteristics provided additional information on local network changes (i.e. hub shifts) after stroke. Although the overall network topology did not significantly differ between young-adult and aged rats, nodal characteristics (such as betweenness centrality and nodal strength) revealed hub-shifts with ageing (chapter 5). This information on (changes in) nodal characteristics will be lost with the use of generative modelling or motif count approaches, showing the value of classical graph metrics.

In order to solve the intrinsic limitations of classical graph analysis, novel network analysis methods have been developed, such as the generative Bayesian ERGM (Caimo and Friel, 2011; Robins et al., 2007a; Simpson et al., 2011) and the descriptive MST approach (Stam et al., 2014; Tewarie et al., 2015a). We applied the MST approach to analyse structural brain networks chronically after stroke (chapter 4). The MST approach takes a subset of all nodes, forming a new graph that connects all the nodes from the original network but does not contain any cycles or loops (i.e. the network backbone) (Jackson and Read, 2010). Thereby it minimizes the sum of the costs of all edges over the set of all possible MSTs that could be constructed from the original graph (Hidalgo et al., 2007). The usefulness of MST analysis has been shown in previous studies on ageing (Boersma et al., 2013; Otte et al., 2015; Smit et al., 2016), multiple sclerosis (Engels et al., 2015; Tewarie et al., 2015b), epilepsy (van Diessen et al., 2016), which we extended with our studies on deafness (Sinke et al., 2019) and stroke (chapter 4). MSTs seem to able to capture similar network characteristics as classical graph analysis, without being biased by the number of nodes and edges (Tewarie et al., 2015a; van Wijk et al., 2010). Despite the potential of the MST approach for reliable and robust analysis of the network backbone, it is possible that important (or even crucial) information from weaker (or excluded) connections will be lost. For example, if multiple proximal structural connections (which may even form a small subnetwork) are affected by a stroke, one or more of these connections will be excluded from the MST analyses. Furthermore, the MST approach does not allow formation of cycles in the network, thereby excluding an important characteristic of brain networks (e.g. the 'richclub' of highly connected hub regions). These issues may be (partially) solved by starting with a MST and subsequently adding nearest neighbour connections up to a certain density (Alexander-Bloch et al., 2010).

The Bayesian ERGM approach potentially solves the intrinsic limitations of classical graph analyses by simultaneous exploration of multiple local network features (i.e. building blocks), such as local clustering and efficiency, and characterization of how these local building blocks shape the global network topology (i.e. as an emerging property). Thereby it accounts for mutual dependencies and resolves the bias due to density differences (van 
Wijk et al., 2010). The Bayesian ERGM approach has been successfully applied to assess brain network topology (Simpson et al., 2011, 2012). Correspondingly, we were able to characterize structural networks across the lifespan, showing topological characteristics such as global efficiency and local clustering (chapter 2), in accordance with previous research (Bullmore and Sporns, 2009). Interestingly, the local features that give rise to the overall network topology were stable across the lifespan, contrary to previous studies using descriptive network approaches, which showed structural and functional network alterations across the lifespan (Dennis et al., 2013; Meunier et al., 2009; Montembeault et al., 2012; Otte et al., 2015; Smit et al., 2016; Spreng and Schacter, 2012). This was even the case after simulations of lesions in random or hub nodes (although some trends of deviation were apparent), contrary to findings from previous studies using conventional graph analysis tools (Alstott et al., 2009) (chapter 5). The relatively stable contribution of local network structures that give rise to the global network topology across the lifespan, which remained after different degrees of damage, may indicate a robust hierarchical organization using similar patterns or motifs. A drawback of (Bayesian) ERGM models is the high computational load, limiting the maximum number of nodes and edges. Furthermore, the Bayesian ERGM approach we used was not able to handle weighted brain networks. This may be solved by the application of generalized ERGM approaches (Stillman et al., 2017).

Results from this thesis and from previous brain network research show that every network analysis approach has its own strengths and limitations. Different approaches should be viewed as complementary rather than mutually exclusive. Every approach can provide valuable information on local and global network topology characteristics (chapters 4 and 5), or on underlying mechanisms and features that drive the network topology (chapter 2). Future studies should combine multiple network analysis approaches to characterize (changes in) networks with complementary information. In addition, these studies could also employ more complex network modelling techniques, such as dynamic functional and effective connectivity analyses as well as modelling of brain communication dynamics, which may serve as link between structural and functional network topology (i.e. structure-function relation) (Avena-Koenigsberger et al., 2017; Bassett et al., 2018). Furthermore, these studies should also include control and manipulation of brain networks, as well as integration across different spatiotemporal levels (Bassett and Sporns, 2017). Together, this will further improve our understanding of structural and functional brain connectivity in health and disease.

\section{Concluding thoughts}

Over the last decades brain network science revealed many new insights into the organization of structural and functional brain networks in health and disease. In this thesis we investigated the brain from a network perspective in order to characterize 
spatiotemporal network reorganization after brain damage as well as to study the effect of ageing on brain network vulnerability. Thereby we focused on global structural and functional network topology as well as on specific connections in the brain. The presented studies suggest stable global structural and functional network topology across the lifespan, although local network characteristics differ as indicated by significant hub shifts. Furthermore, the effect of lesions seems to be larger in aged rats and humans, indicating increased vulnerability to brain damage with ageing. Experimental unilateral stroke affected the structural and functional network topology, even in the contralesional hemisphere, resulting in reduced network efficiency. Changes in nodal characteristics and specific connections may indicate plasticity and compensatory mechanisms. Furthermore, cortical stroke induced remote alterations in the CST up to chronic time points. Behavioural recovery, despite chronic CST alterations, may indicate dependency on intact transcallosal tracts and restoration of interhemispheric functional connectivity. In another study we found that experimental TBI induces alterations in several white matter structures. These restored over time, which coincided with behavioural recovery after initial impairment, further emphasizing the importance of white matter tract integrity for appropriate brain (and behavioural) functioning. Together the studies in this thesis show the potential of the network analytical approach to investigate the brain in health and disease, the application of which may continuously lead to new insights in disease mechanisms, identification of diagnostic biomarkers as well as detection of therapeutic targets. 


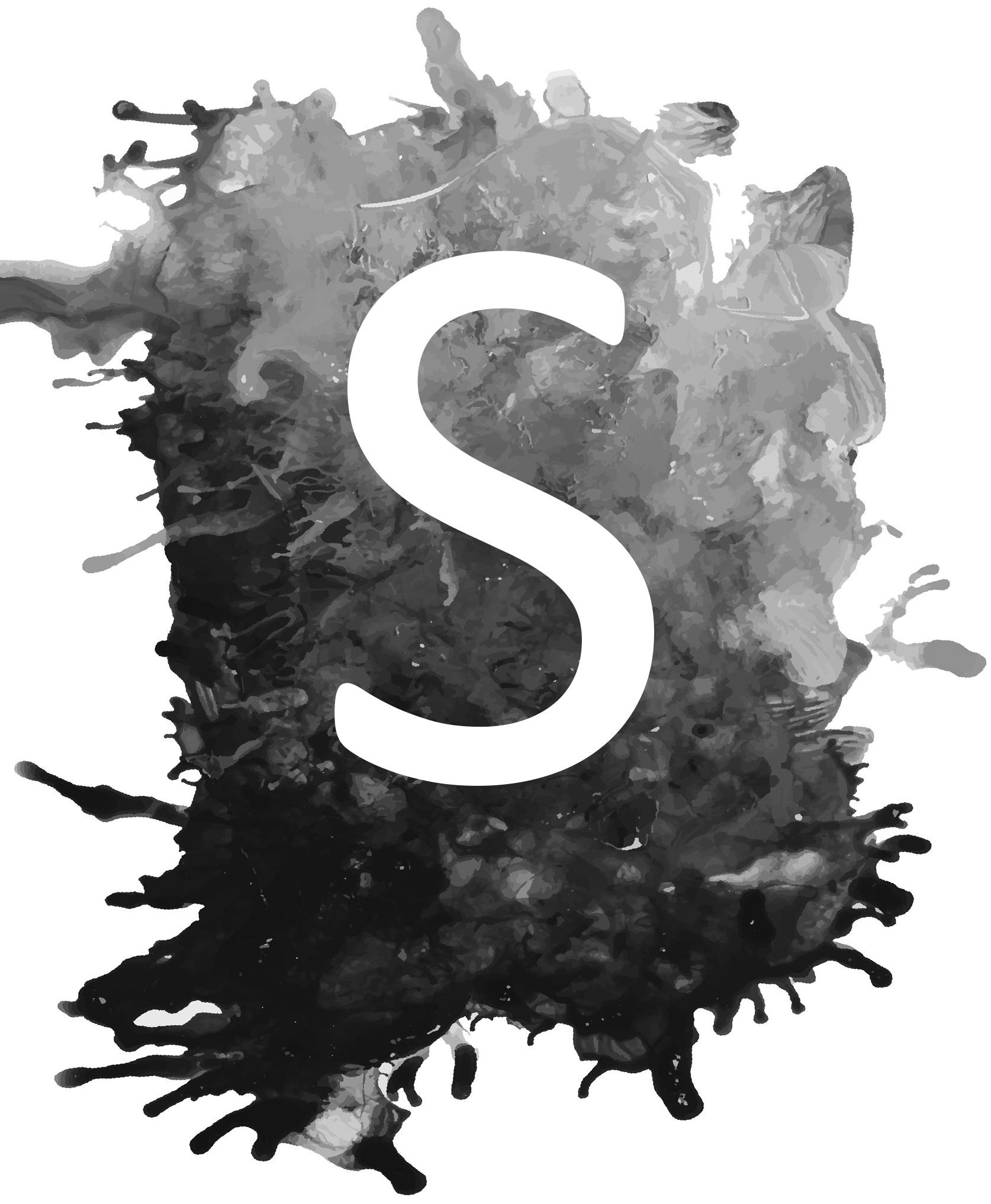


Supplementary Figures 



\section{CHAPTER 2}

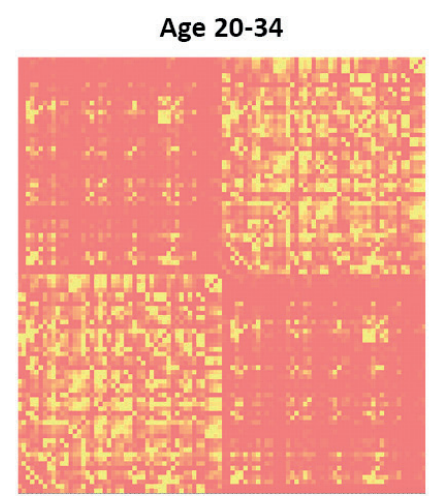

Age 51-70

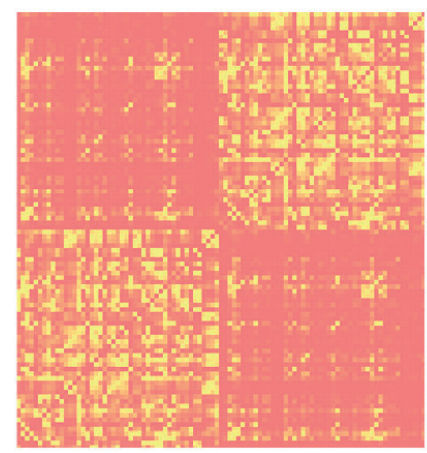

Age 35-50

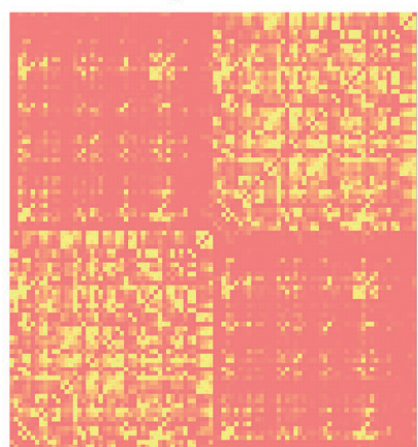

Age $>70$

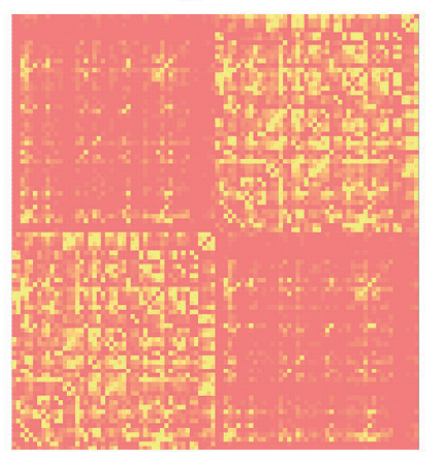

Figure S1. Average matrices for all age categories with edge-weights based on edge-presence in individual networks. All binary individual matrices were summed and divided by the total number of matrices (individuals) for each age category. Each pixel shows the proportion of participants having a specific edge, from 0 (red) to 1 (yellow). 
Age 20-34

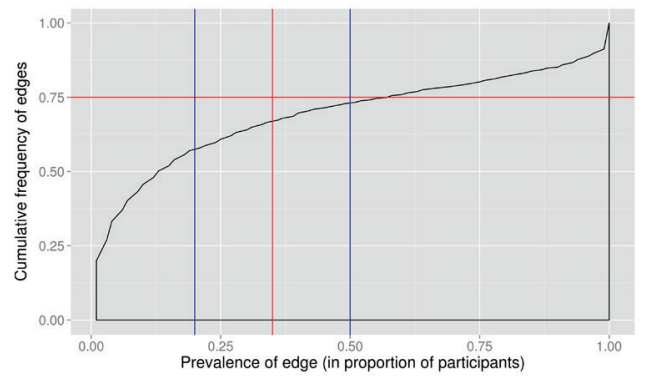

Age 35-50

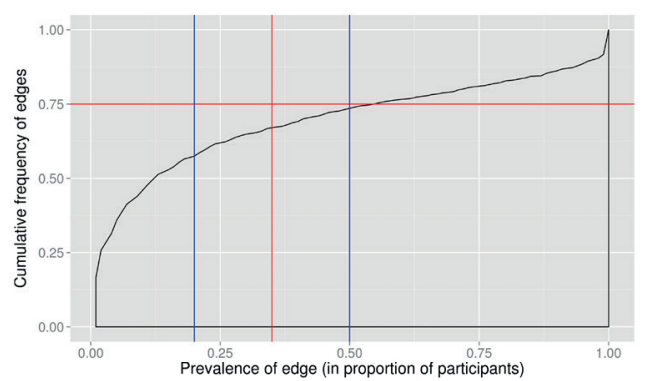

Age 51-70

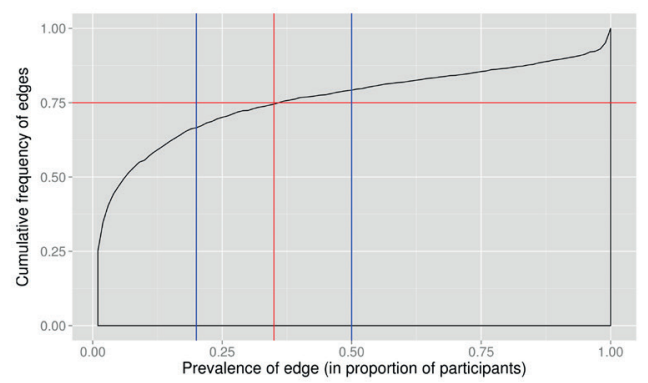

Age $>70$

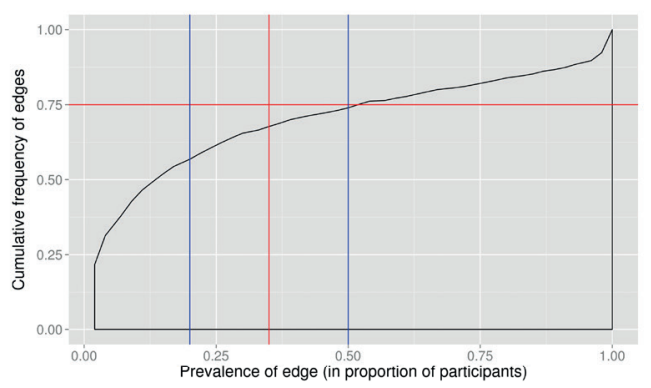

Figure S2. Cumulative frequencies of edge prevalence for each age category matrix. The edge prevalence is represented by the proportion of participants having a specific connection ( $x$ axis). We used a threshold of 0.35 (vertical red line), which means that at least $35 \%$ of the participants must have a specific edge to be included in the age group network. With this threshold at least $25 \%$ of the prevalent edges are included, which can be seen from the horizontal red cutoff line at 0.75 (cumulative frequency). The blue vertical lines at 0.20 and 0.50 represent the other thresholds we used for additional analyses, which would lead to inclusion of more or less connections, respectively. 
Age 20-34

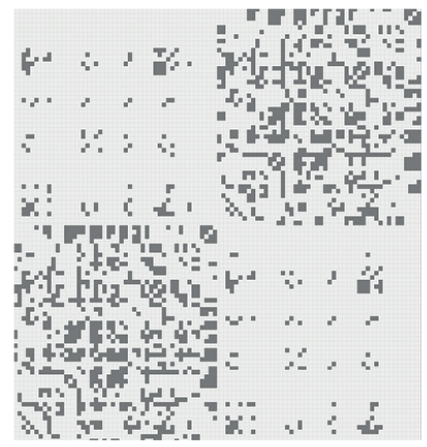

Age 51-70

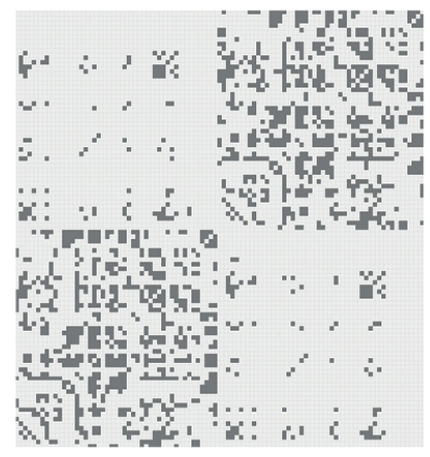

\section{Age 35-50}

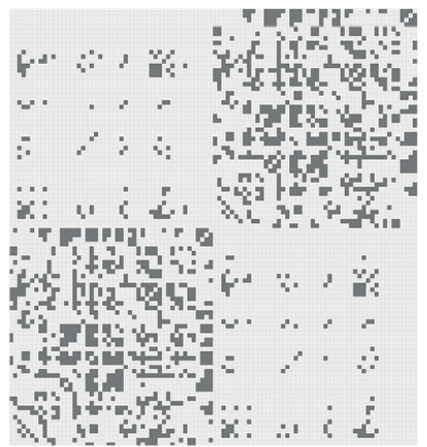

Age $>70$

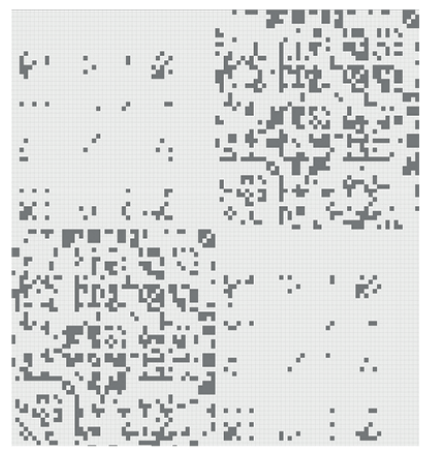

Figure S3. Binary connectivity matrices for all age categories with threshold $\mathbf{0 . 3 5}$. Weighted (average) group matrices (see Figure S1) were binarized using a threshold of 0.35, meaning that at least $35 \%$ of the participants have a connection for a given node-pair that has a connection in these binary matrices. 
Age 20-34

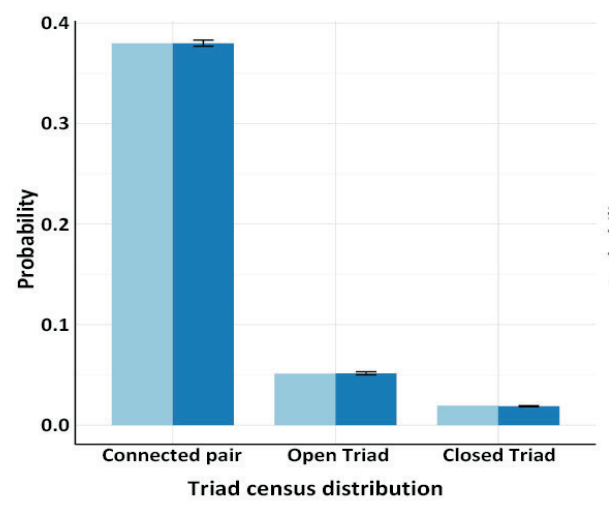

Age $51-70$

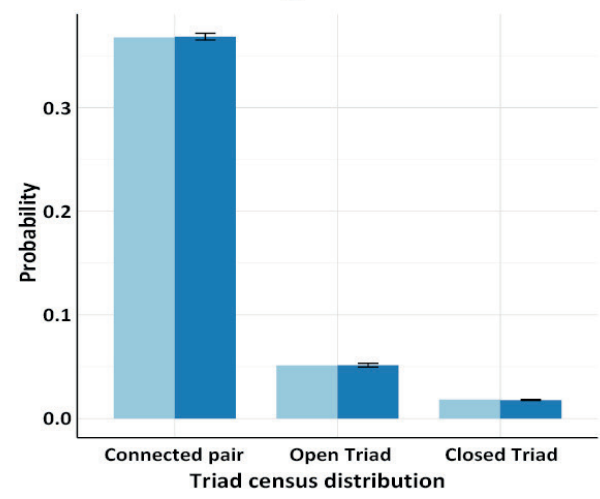

Age 35-50

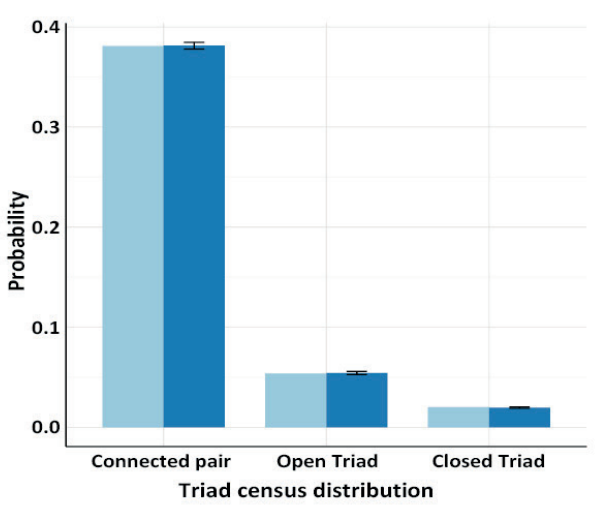

Age $>70$

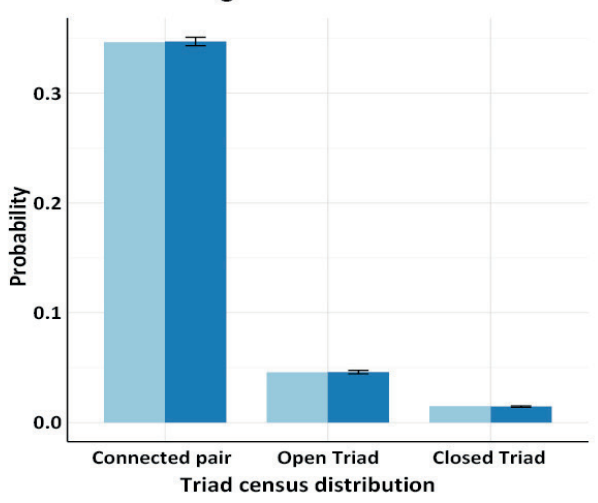

Figure S4. Goodness-of-fit based on comparison of triad census. Bayesian goodness-of-fit statistics for observed and simulated networks for all four age categories. The probability of three Triad Census parameters were compared between simulated (dark blue) and observed (light blue) networks. The dark blue bar-graphs represent averages of simulated networks, drawn from the posterior distribution plotted as mean $\pm 2 \times$ standard deviation. 
Age 20-34

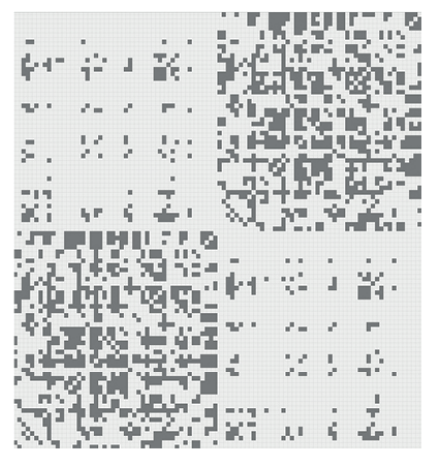

Age 51-70

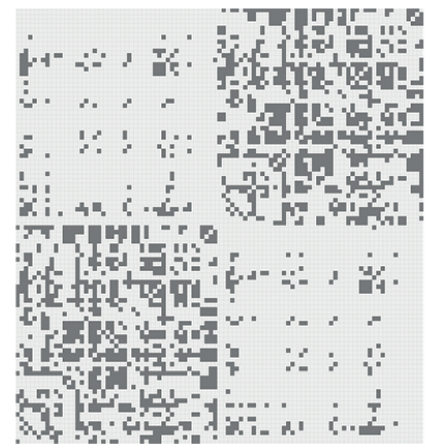

Age 35-50

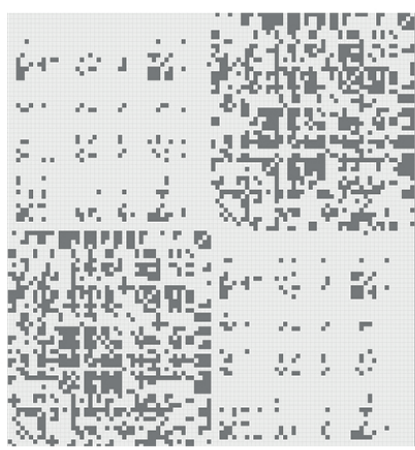

Age $>70$

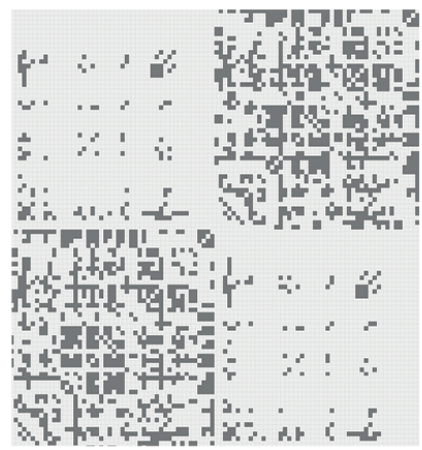

Figure S5. Binary connectivity matrices for all age categories with threshold 0.20. Weighted (average) group matrices (see Figure S1) were binarized using a threshold of 0.20, meaning that at least $20 \%$ of the participants have a connection for a given node-pair that has a connection in these binary matrices. 
Age 20-34

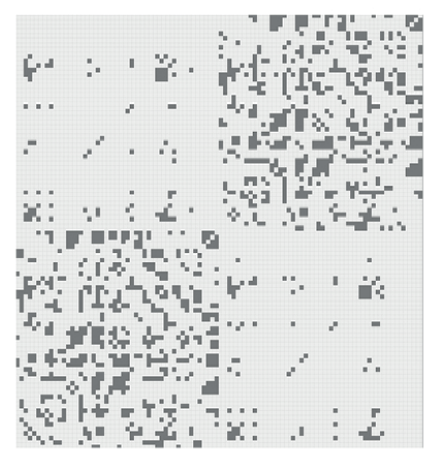

Age 51-70

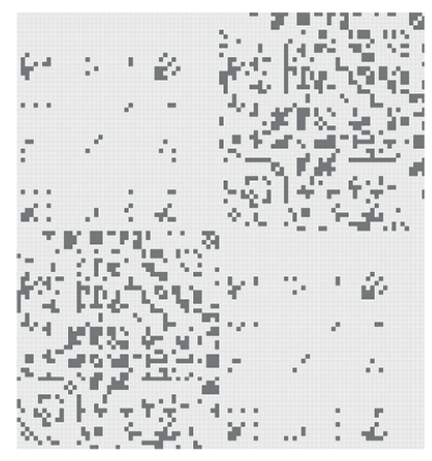

Age 35-50

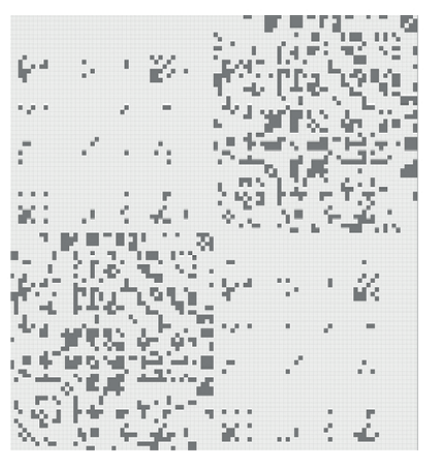

Age $>70$

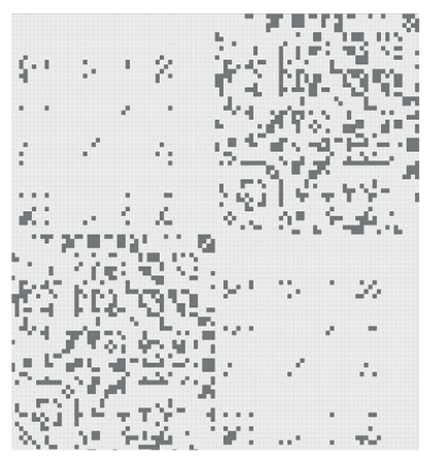

Figure S6. Additional binary connectivity matrices for all age categories with threshold $\mathbf{0 . 5 0}$. Weighted (average) group matrices (see Figure S1) were binarized using a threshold of 0.50 , meaning that at least $50 \%$ of the participants have a connection for a given node-pair that has a connection in these binary matrices. 


\subsection{0}
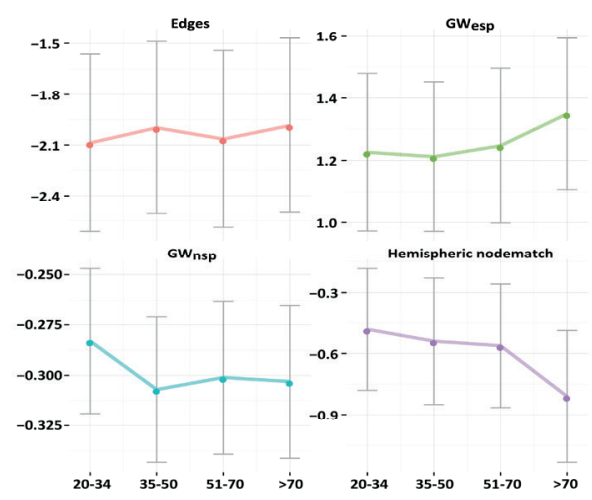

Age categories

\subsection{0}
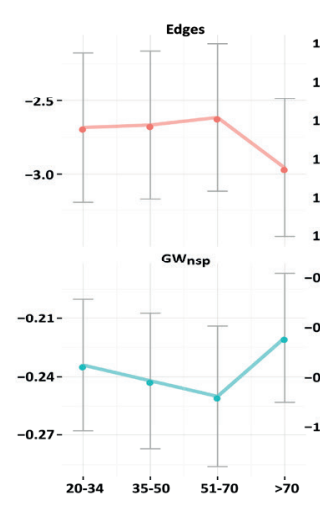

Homisphoric nodomatch

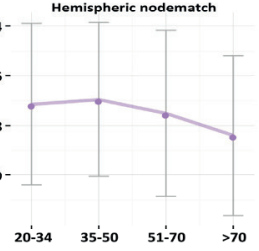

Age categories

Figure S7. Modeling results at different group-level network sparsities. Edges, GWESP, GWNSP, and nodematch hemisphere coefficients for age categories 20-34, 35-50, 51-70, and >70 years of age are shown for different network sparsities. Sparsities correspond to binarizing thresholds of 0.20 and 0.50 used in the construction process of the group-level networks. Results in the main text are based on a threshold of 0.35 . 


\section{CHAPTER 3}

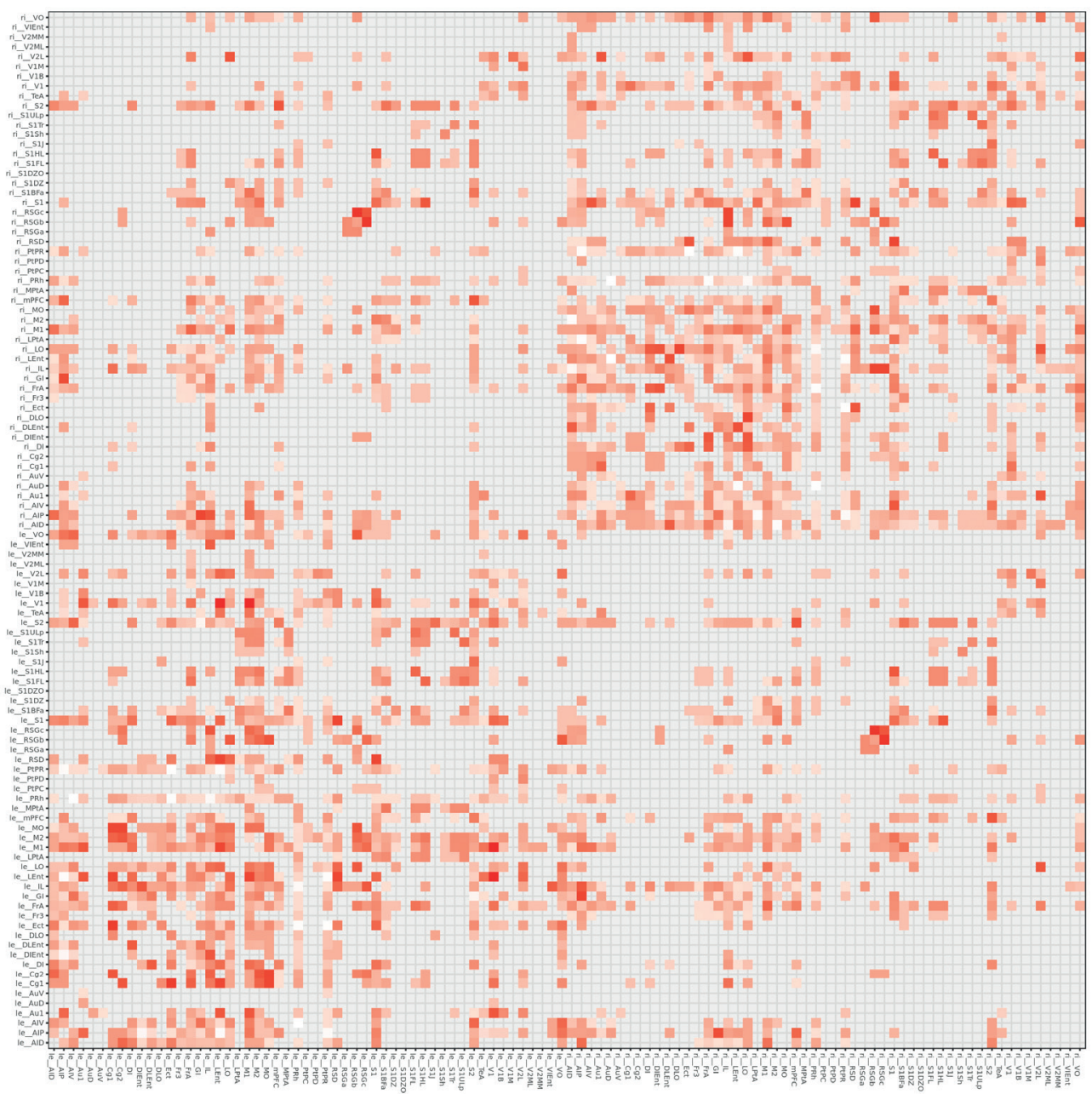

Figure S1. Weighted neuronal tracer-based connectivity matrix. The weighted neuronal tracer-based connectivity matrix (consists of rows and columns representing 106 cortical regions and revealed a network density of 0.27 . Connectivity weights are based on the strength of histological connections, ranging from 1 (white) to 8 (red). 'le_[Name]' indicates a node in the left hemisphere, 'ri_[Name]' indicates a node in the right hemisphere. 

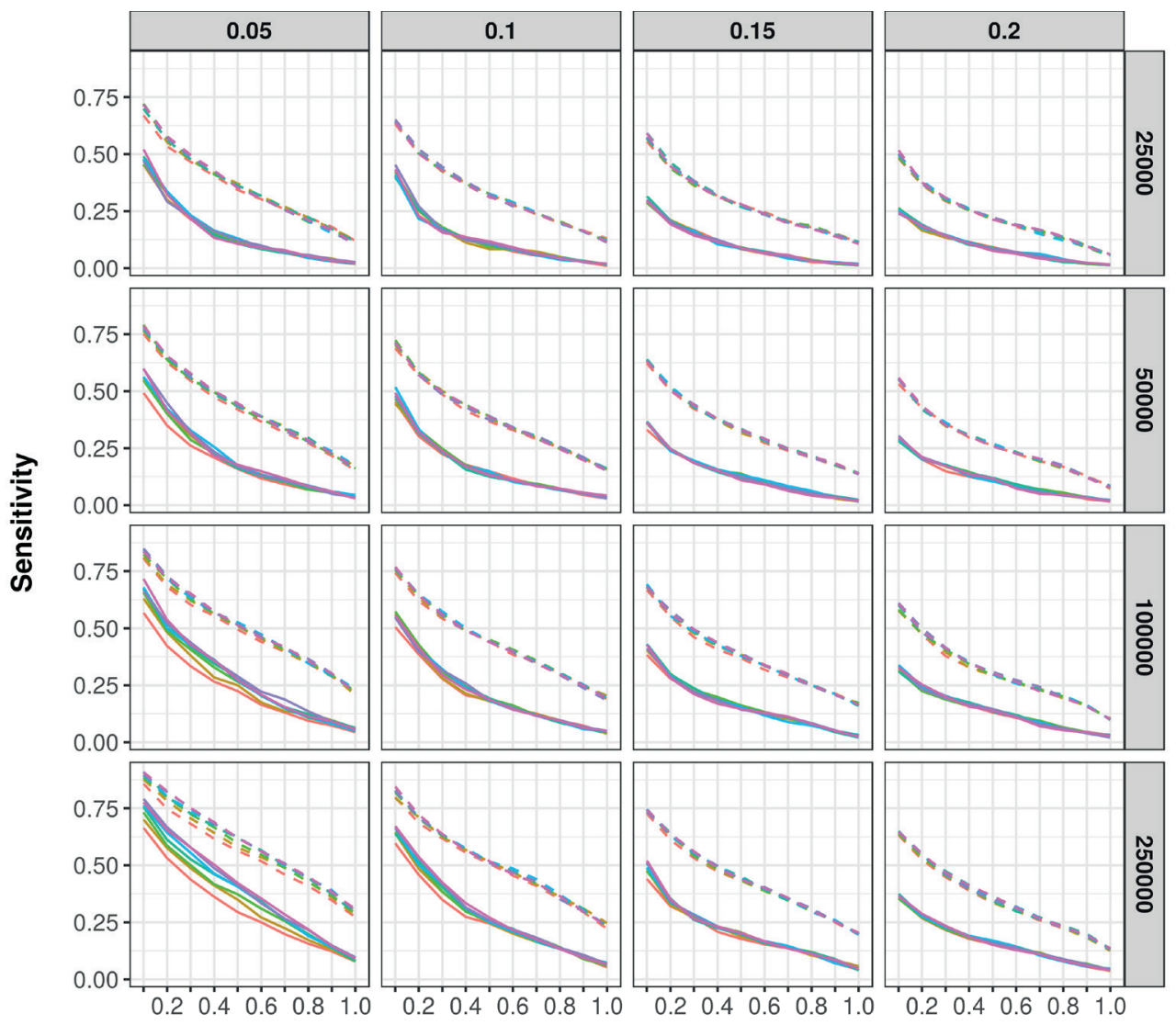

Incidence threshold

Figure S2. Effect of group-based network thresholding on reconstruction sensitivity of DT-based tractography. Sensitivity is shown for interhemispheric (solid lines) and intrahemispheric (dashed lines) connections. The network proportion threshold (x-axis) reflects the proportion of rats with a specific connection. Sensitivity is shown over different FA thresholds (columns), number of streamlines (rows) and different angle thresholds (line colour). 

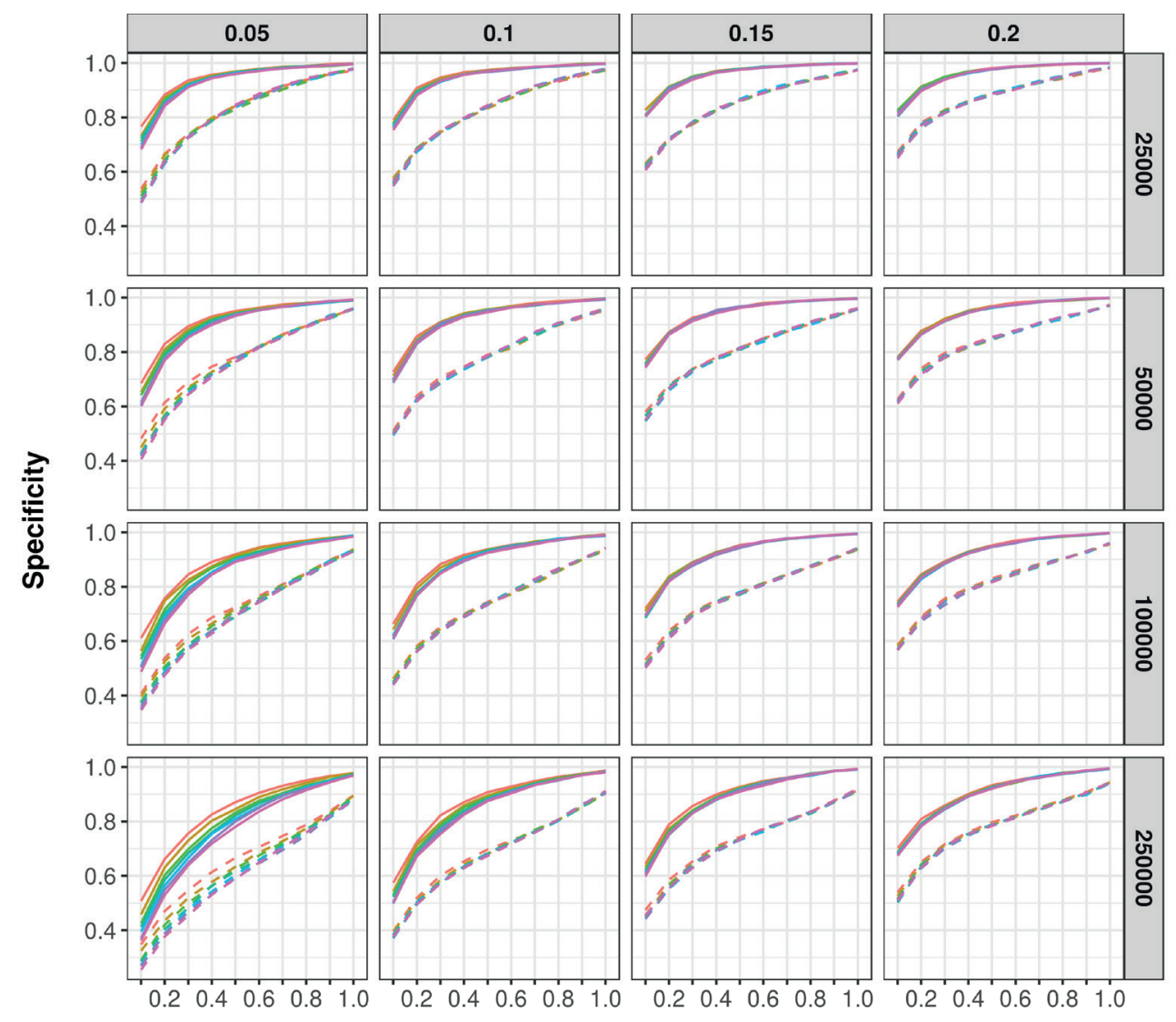

Incidence threshold

Figure S3. Effect of group-based network thresholding on reconstruction specificity of DT-based tractography. Specificity is shown for interhemispheric (solid lines) and intrahemispheric (dashed lines) connections. The network proportion threshold (x-axis) reflects the proportion of rats with a specific connection. Sensitivity is shown over different FA thresholds (columns), number of streamlines (rows) and different angle thresholds (line colour). 

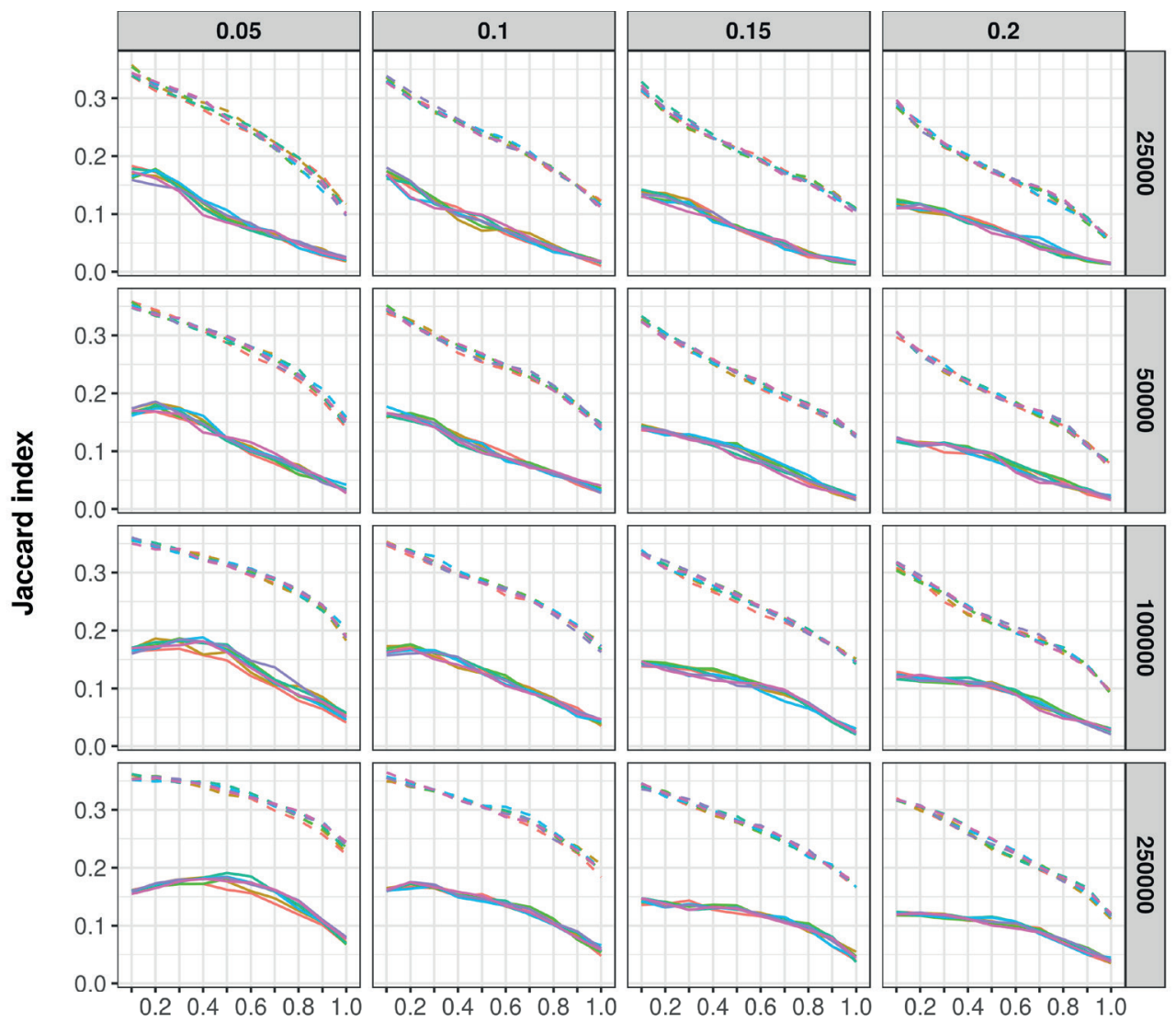

Incidence threshold

Figure S4. Effect of group-based network thresholding on reconstruction Jaccard index of DT-based tractography. Jaccard index is shown for interhemispheric (solid lines) and intrahemispheric (dashed lines) connections. The network proportion threshold ( $x$-axis) reflects the proportion of rats with a specific connection. Sensitivity is shown over different FA thresholds (columns), number of streamlines (rows) and different angle thresholds (line colour). 

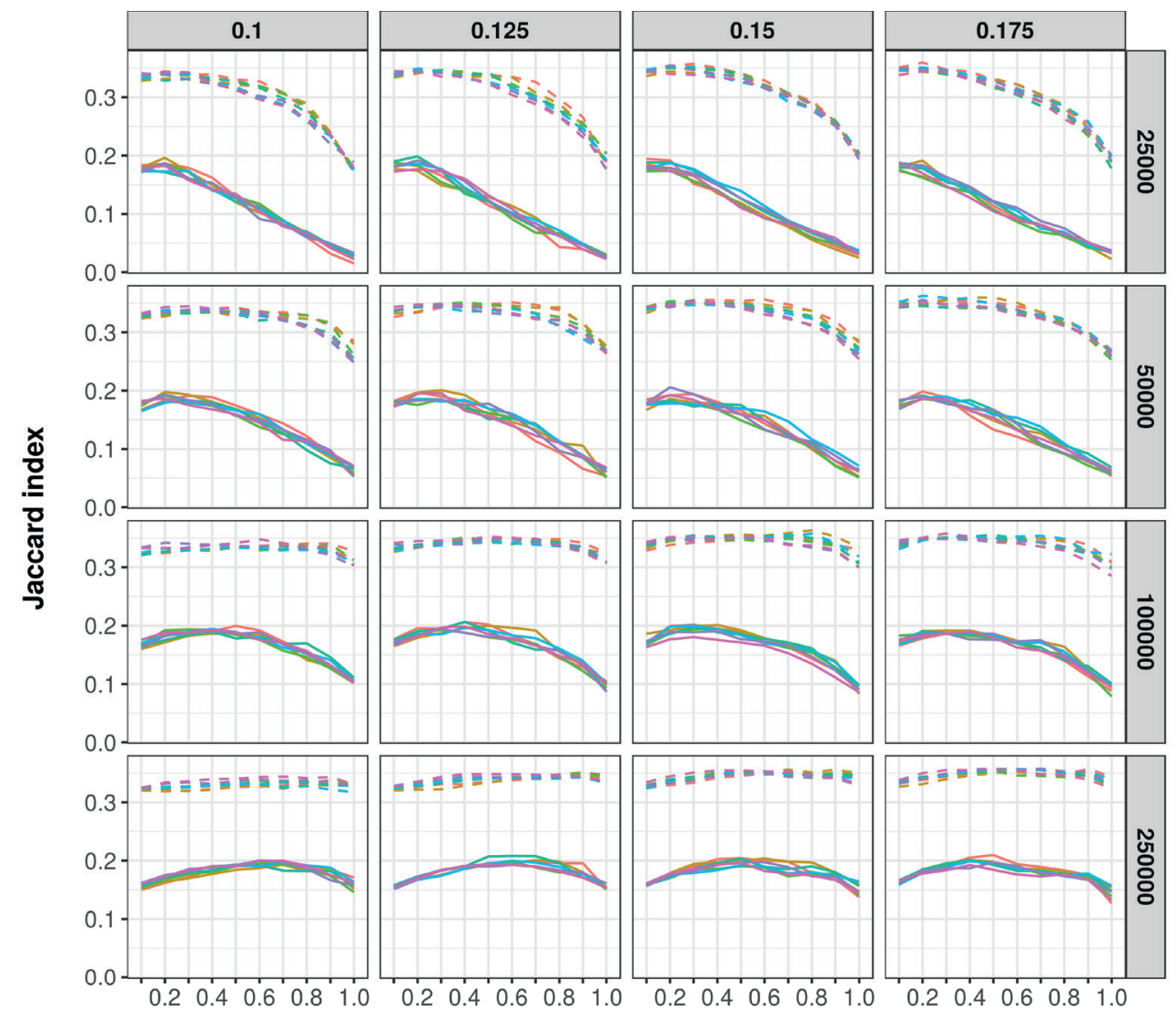

Incidence threshold

Figure S5. Effect of group-based network thresholding on reconstruction Jaccard index of CSD-based tractography. Jaccard index is shown for interhemispheric (solid lines) and intrahemispheric (dashed lines) connections. The network proportion threshold (x-axis) reflects the proportion of rats with a specific connection. Sensitivity is shown over different FOD thresholds (columns), number of streamlines (rows) and different angle thresholds (line colour). 


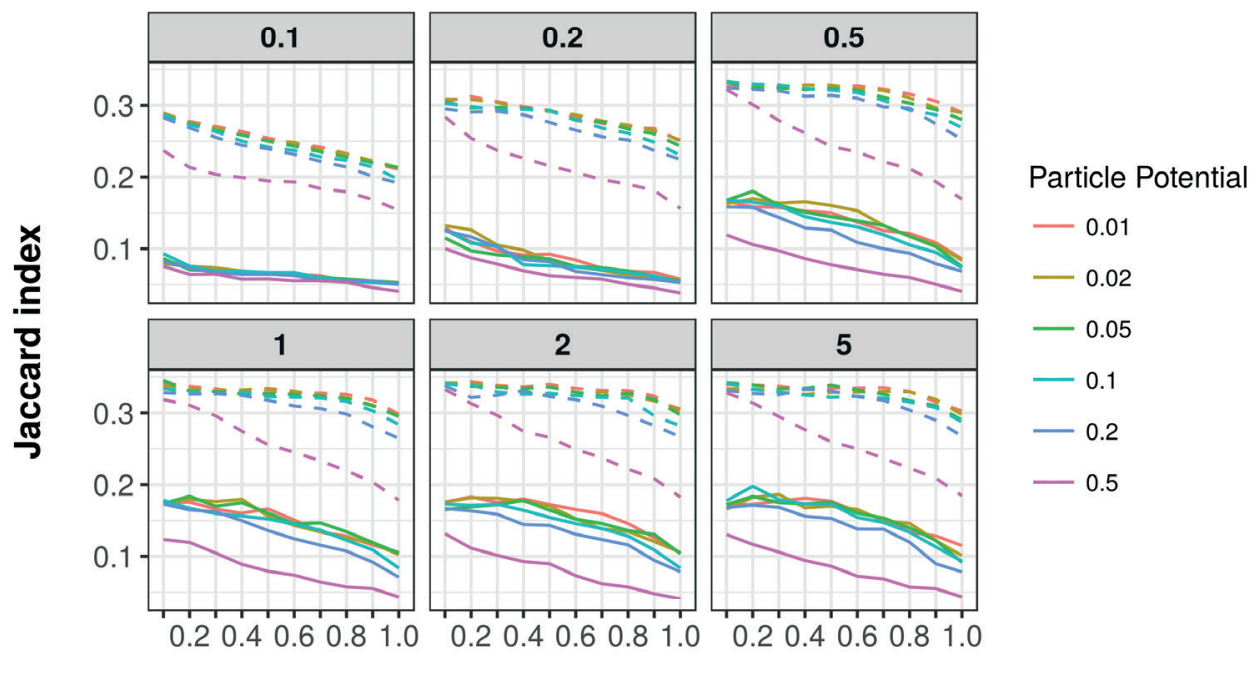

Incidence threshold

Figure S6. Effect of group-based network thresholding on reconstruction Jaccard index of GT-based tractography. Jaccard index is shown for interhemispheric (solid lines) and intrahemispheric (dashed lines) connections. The network proportion threshold ( $x$-axis) reflects the proportion of rats with a specific connection. Sensitivity is shown over different connection potentials (top) and different particle potentials (line colour). 


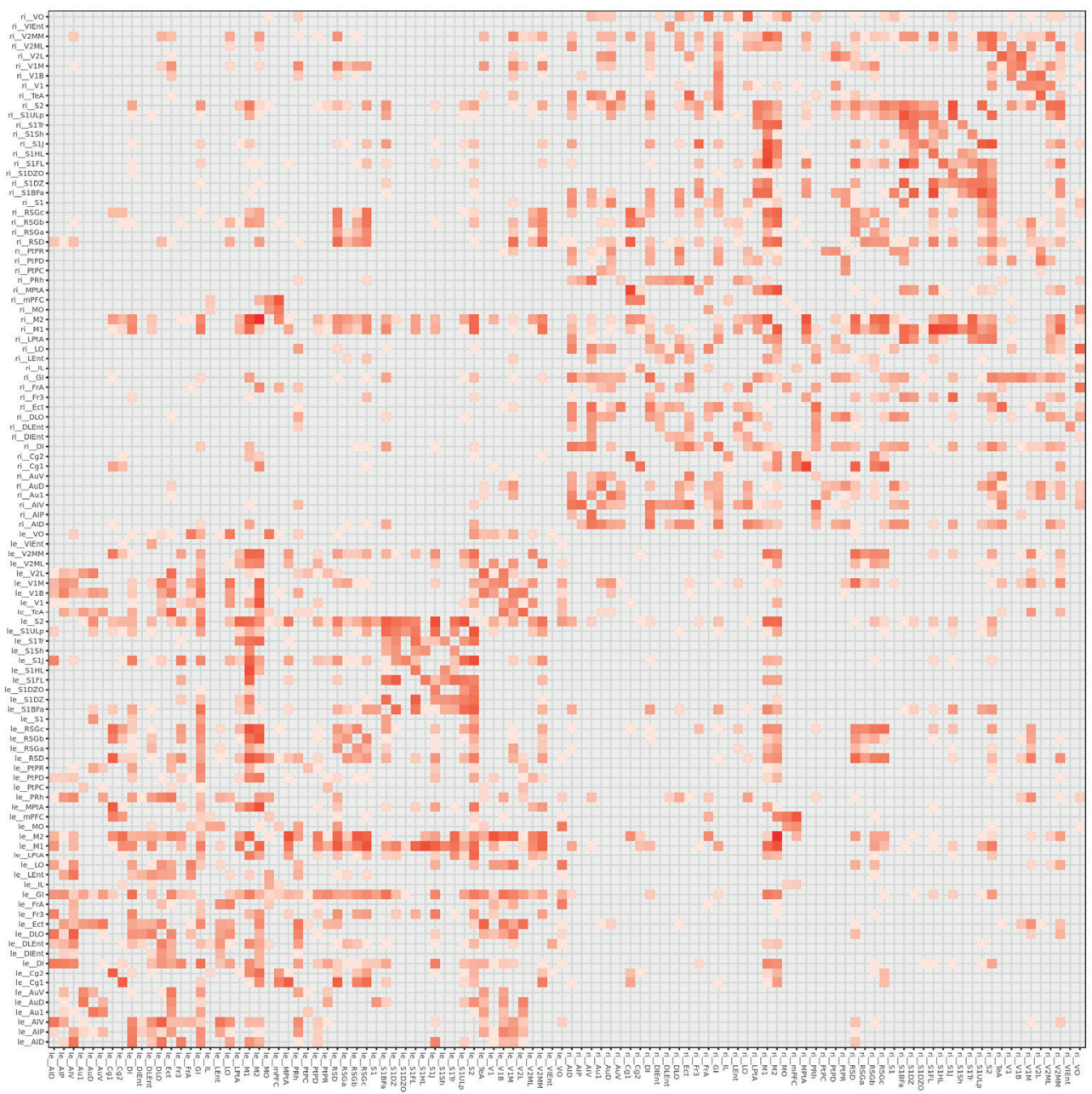

Figure S7. Weighted DT-based connectivity matrix. A structural connectivity matrix in which the rows and columns represent cortical regions $(N=106)$. Connectivity weights are based on the logtransformed number of streamlines connecting two regions, ranging from transparent (no streamlines) to dark red (maximum number of streamlines). 'le_[Name]' indicates a node in the left hemisphere, 'ri__Name]' indicates a node in the right hemisphere. DT parameter settings for this particular matrix (density $=0.22$ ): FA threshold $=0.15$, angle threshold $=40^{\circ}$, step-size $=15 \mu \mathrm{m}$, number of streamlines $=250,000$. 


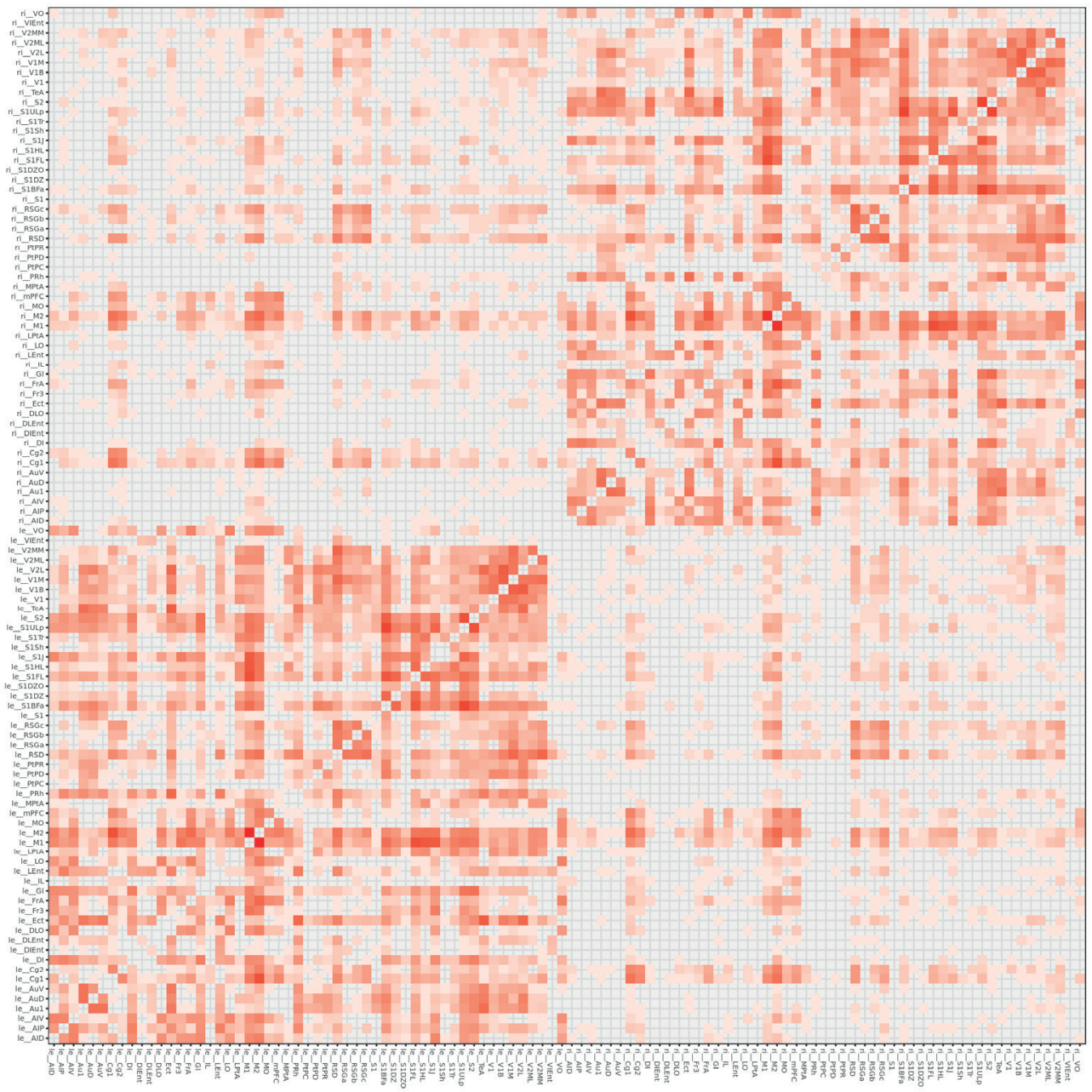

Figure S8. Weighted CSD-based connectivity matrix. A structural connectivity matrix in which the rows and columns represent cortical regions $(N=106)$. Connectivity weights are based on the logtransformed number of streamlines connecting two regions, ranging from transparent (no streamlines) to dark red (maximum number of streamlines). 'le_[Name]' indicates a node in the left hemisphere, 'ri_[Name]' indicates a node in the right hemisphere. CSD parameter settings for this particular matrix (density $=0.54$ ): FOD threshold $=0.125$, angle threshold $=40^{\circ}$, step-size $=75 \mu \mathrm{m}$, number of streamlines $=250,000$. 


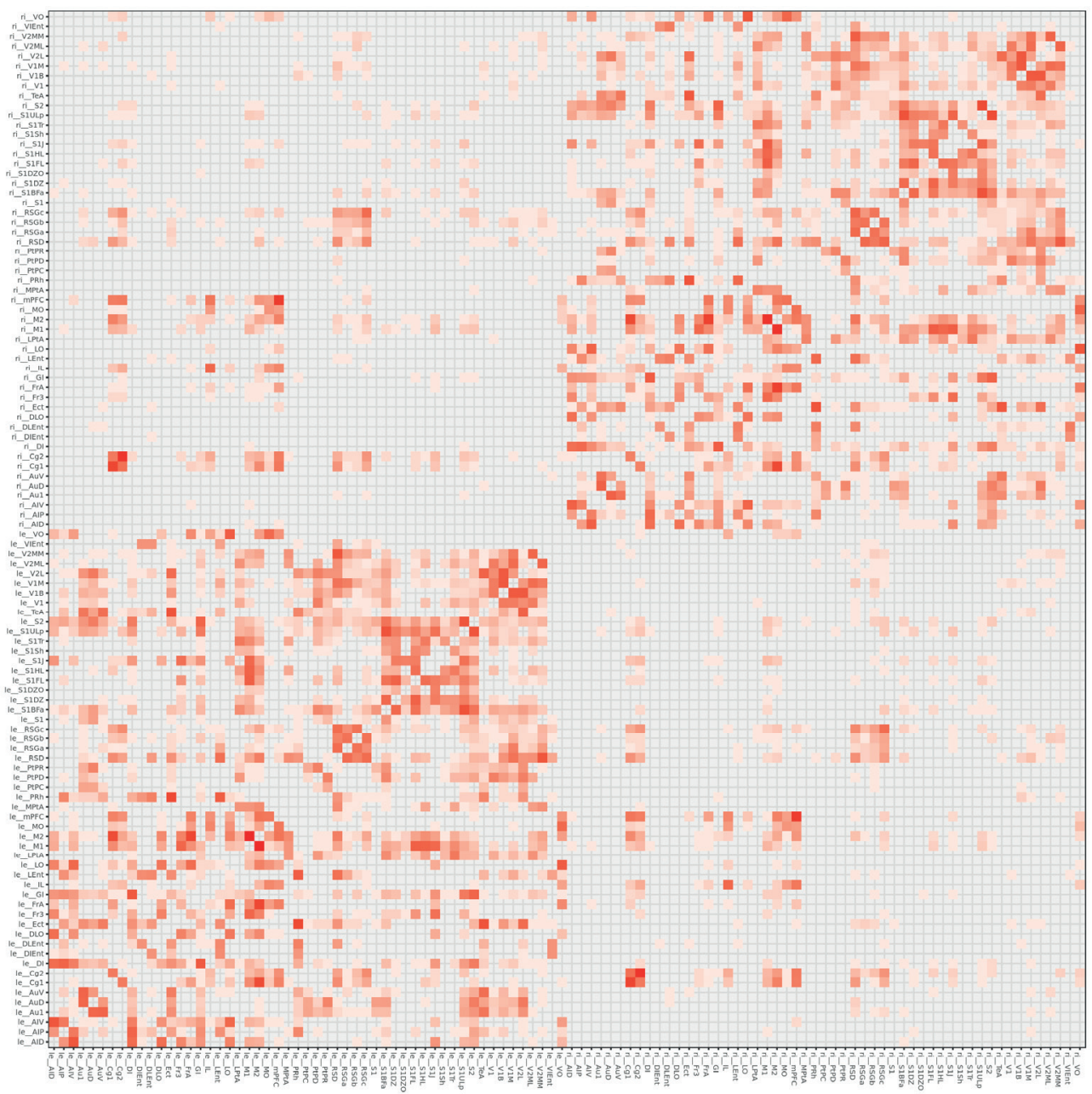

Figure S9. Weighted GT-based connectivity matrix. A structural connectivity matrix in which the rows and columns represent cortical regions $(N=106)$. Connectivity weights are based on the logtransformed number of streamlines connecting two regions, ranging from transparent (no streamlines) to dark red (maximum number of streamlines). 'le_[Name]' indicates a node in the left hemisphere, 'ri_[Name]' indicates a node in the right hemisphere. GT parameter settings for this particular matrix (density $=0.29$ ): particle potential $=0.01$, connection potential $=1$. 


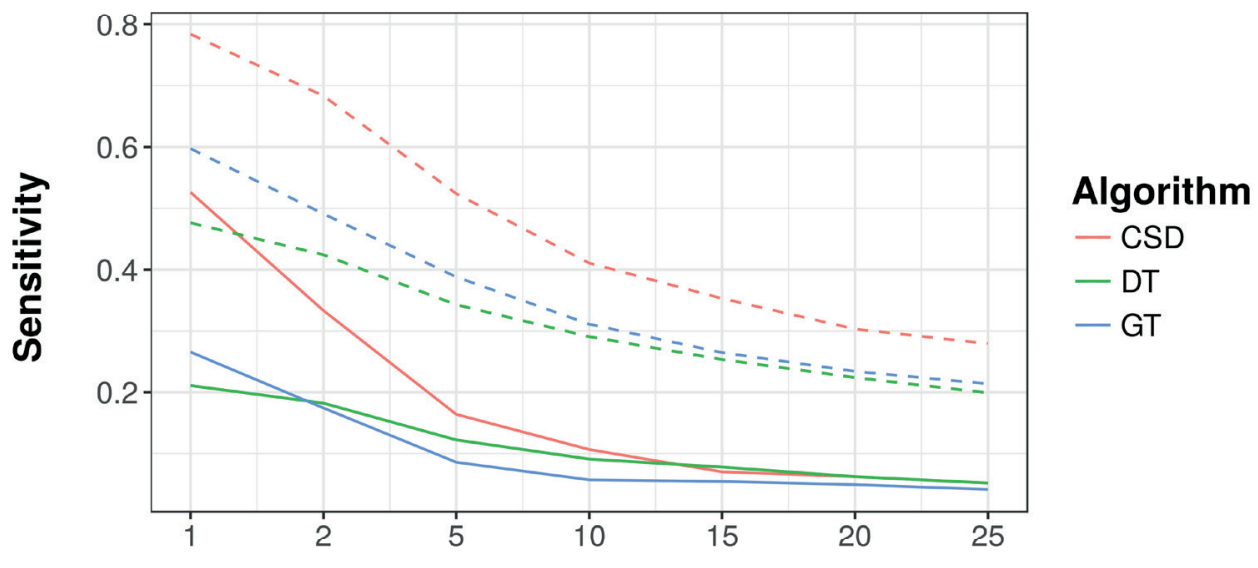

Streamline Threshold

Figure S10. Effect of streamline thresholding on reconstruction sensitivity of DT-, CSD- and GT-based tractography. Sensitivity is shown for interhemispheric (solid lines) and intrahemispheric (dashed lines) connections. The streamline threshold reflects the minimum number of streamlines between two regions to be considered as a connection (i.e. sub-threshold streamline connections were excluded). Parameter settings for DT-, CSD- and GT-based tractography can be found in the legends of Figures S7, S8 and S9, respectively. 


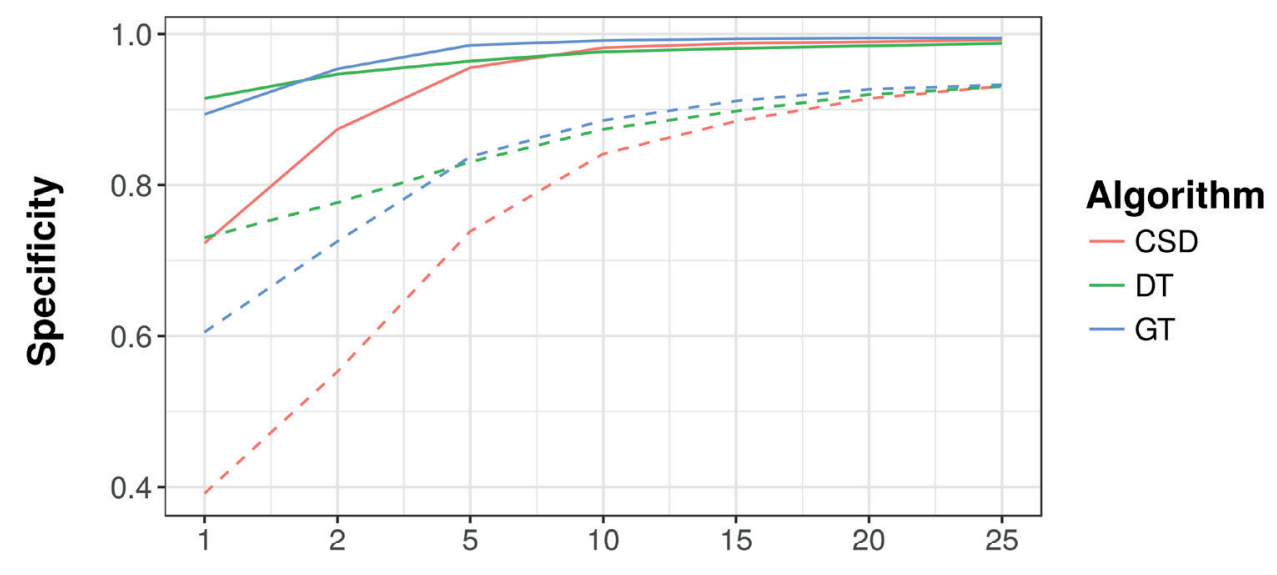

Streamline Threshold

Figure S11. Effect of streamline thresholding on reconstruction specificity of DT-, CSD- and GT-based tractography. Specificity is shown for interhemispheric (solid lines) and intrahemispheric (dashed lines) connections. The streamline threshold reflects the minimum number of streamlines between two regions to be considered as a connection (i.e. sub-threshold streamline connections were excluded). Parameter settings for DT-, CSD- and GT-based tractography can be found in the legends of Figures S7, S8 and S9, respectively. 


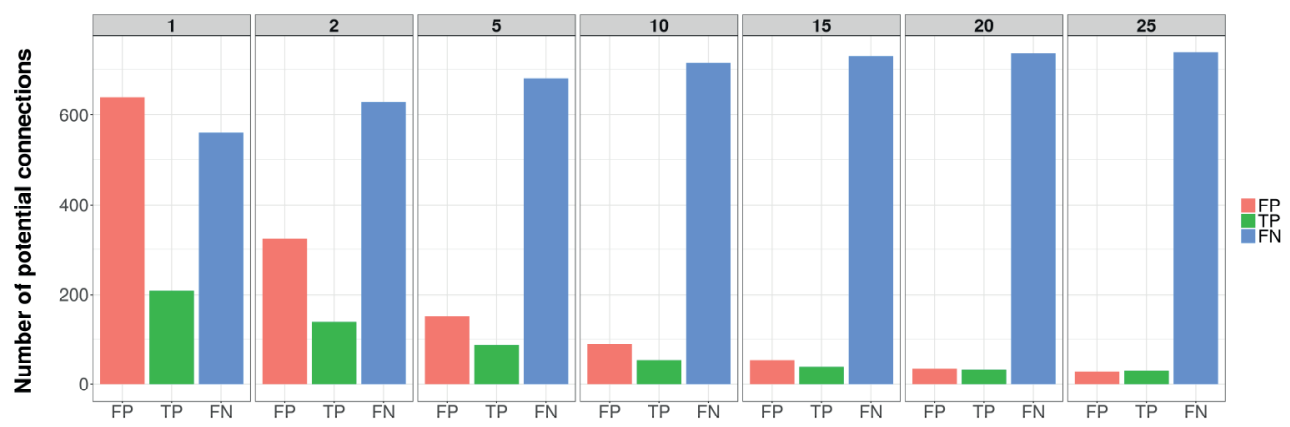

Figure S12. Influence of streamline-thresholds on interhemispheric DT-based tractography reconstruction accuracy. Different streamline thresholds (top) have different effects on the reconstruction accuracy reflected by the number of false positives (FP), true positives (TP) and false negatives (FN) shown at bottom.

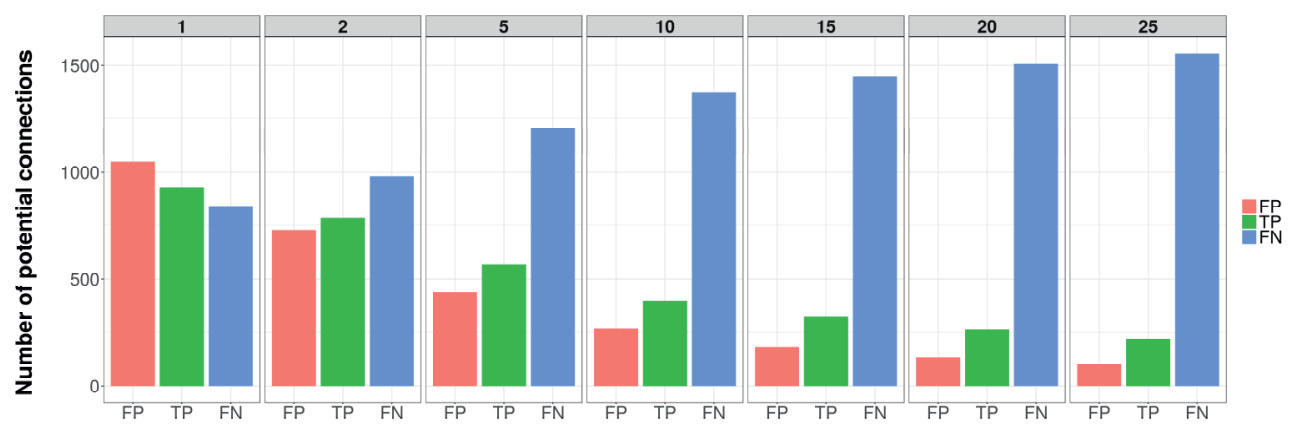

Figure S13. Influence of streamline-thresholds on intrahemispheric DT-based tractography reconstruction accuracy. Streamline threshold (top) affect the accuracy reflected by the number of false positives (FP), true positives (TP) and false negatives (FN) shown at. 


\section{CHAPTER 4}
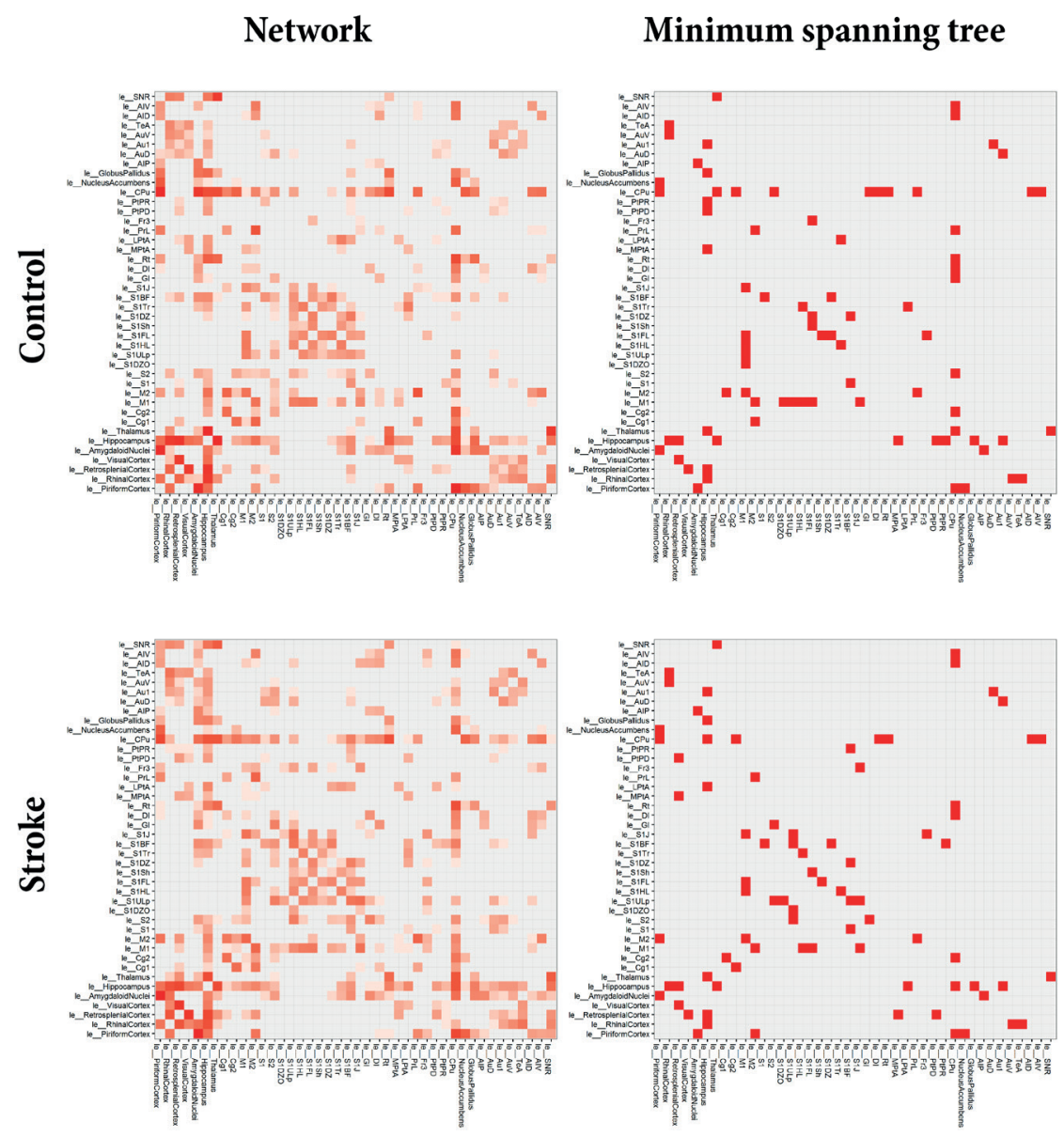

Figure S1. Individual weighted connectivity matrices for the total network and minimum spanning tree. Representative weighted connectivity matrices of total networks (left) and MST backbones (right) of the left (contralesional) hemisphere of a control (top) and a stroke (bottom) animal. Connectivity weights are based on number of tractography streamlines between regions, ranging from low (white) to high (red). 'le_[Name]' indicates node in left (contralesional) hemisphere. 
Diameter

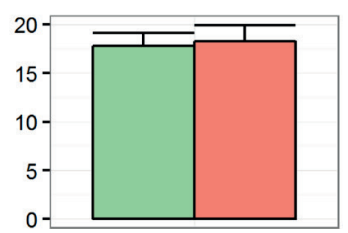

Leafnumber

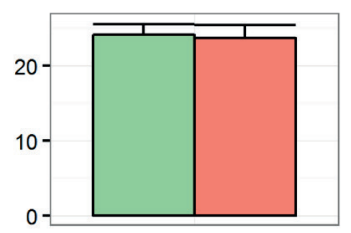

Eccentricity

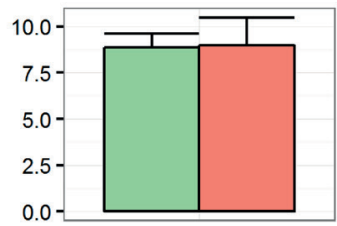

Average BC

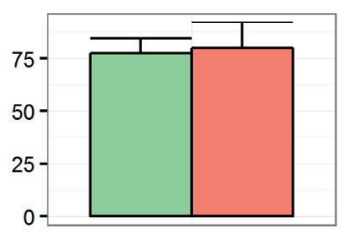

Kappa

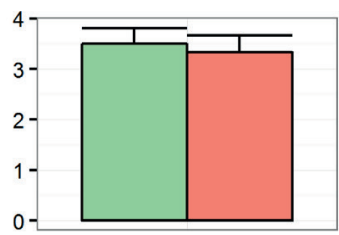

Average strength

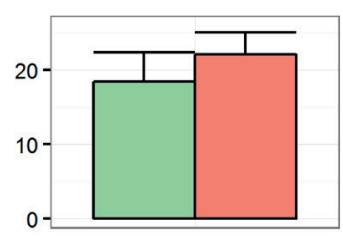

Figure S2. Network backbone characteristics. Network backbone metrics (mean \pm standard deviation) calculated from group-based average MSTs of the left (contralesional) hemisphere in the control (green) and stroke (red) animals. BC: betweenness centrality. 


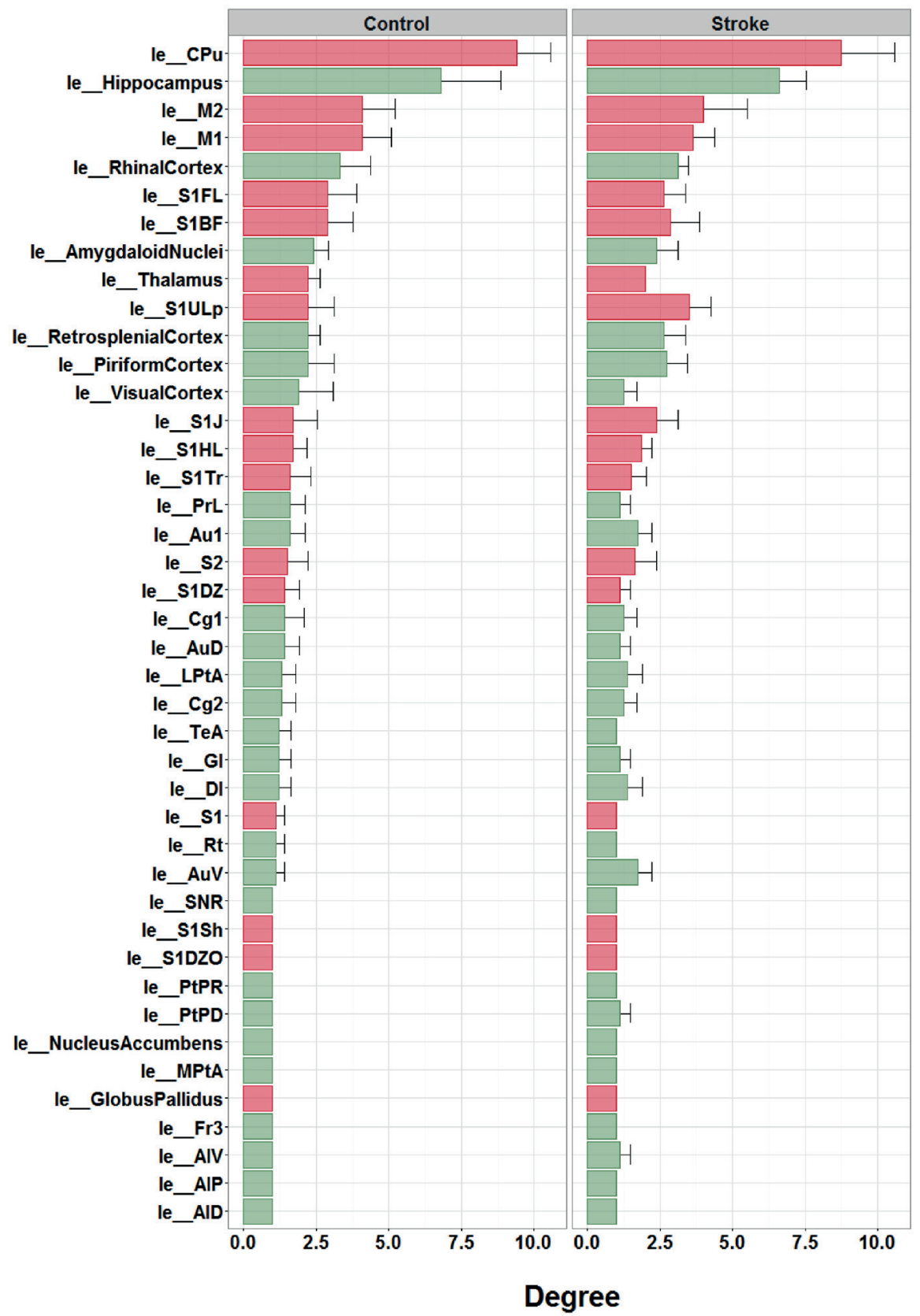

Figure S3. Node degrees from backbone networks. Betweenness centralities (left) and node strength (right) (mean \pm standard deviation) -calculated from MSTs of the left (contralesional) hemisphere in control and stroke animals- ranked from high to low (based on control group data). 'le_[Name]' indicates brain atlas region in left (contralesional) hemisphere. Red bars represent sensorimotor regions. 

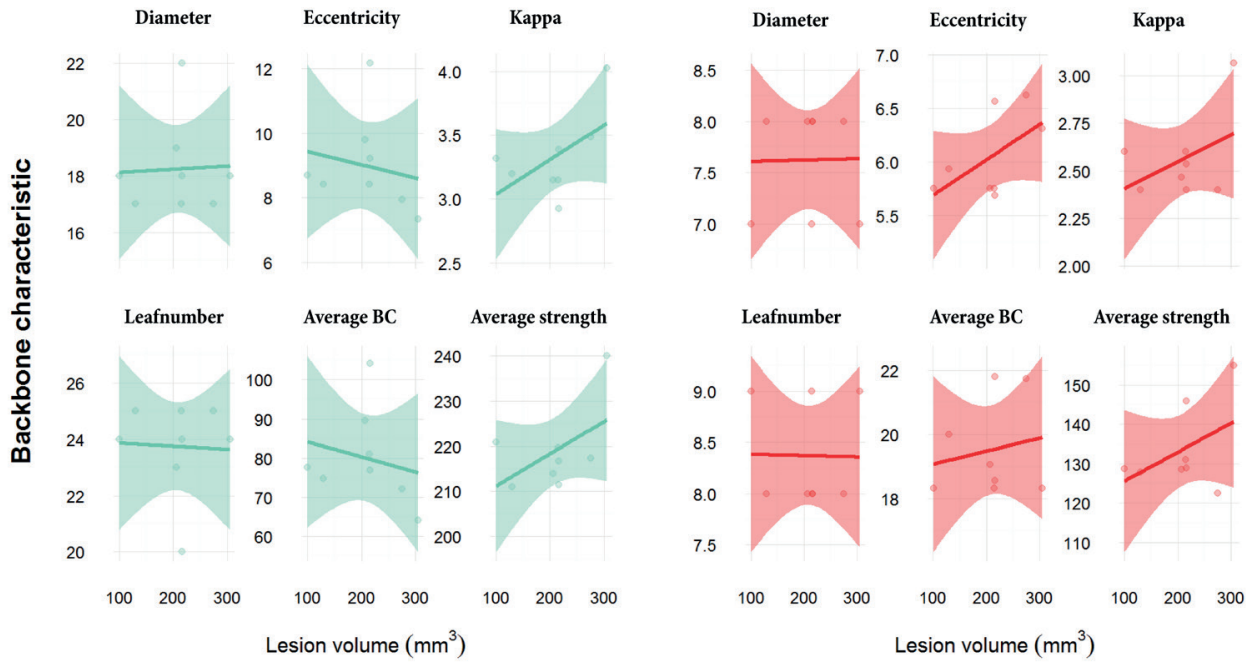

Figure S4. Contralesional MST metrics versus lesion volume. Linear model fits of MST metrics versus lesion volume for the total structural network (left, green) and the sensorimotor network (right, red) in the contralesional hemisphere (transparent bands indicate standard deviation). BC: betweenness centrality. 
CHAPTER 5

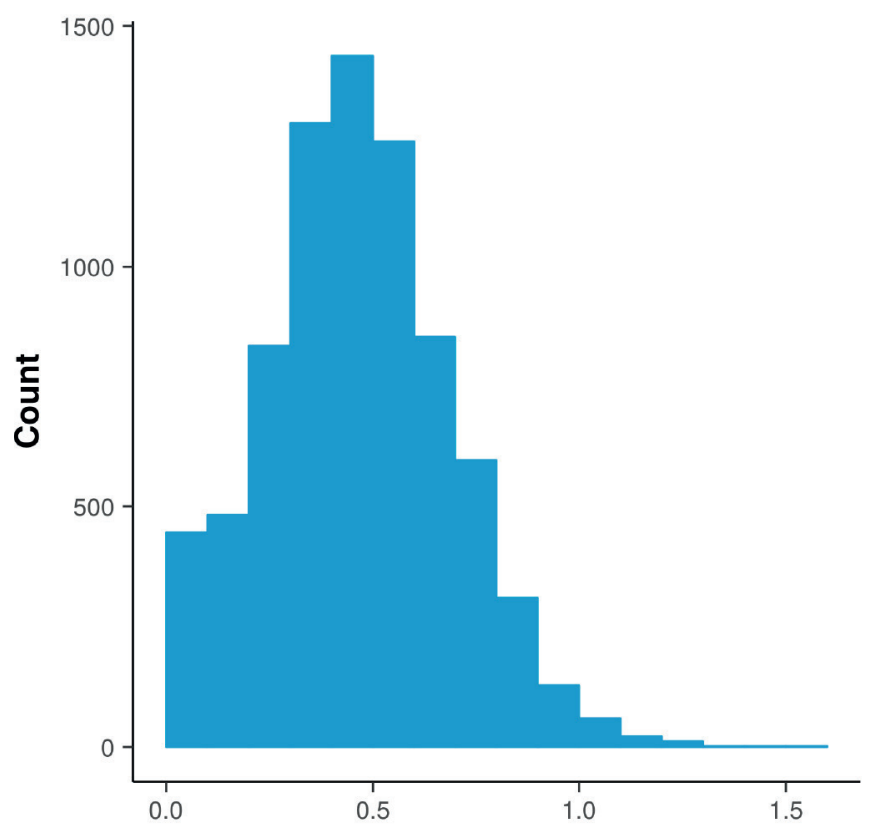

Fisher's Z functional connectivity strength

Figure S1. Distribution of Fisher's Z transformed functional connectivity strength within the brain of a single animal. Functional connectivity strength values are skewed towards lower Fisher's $Z$ transformed correlation coefficient values. 


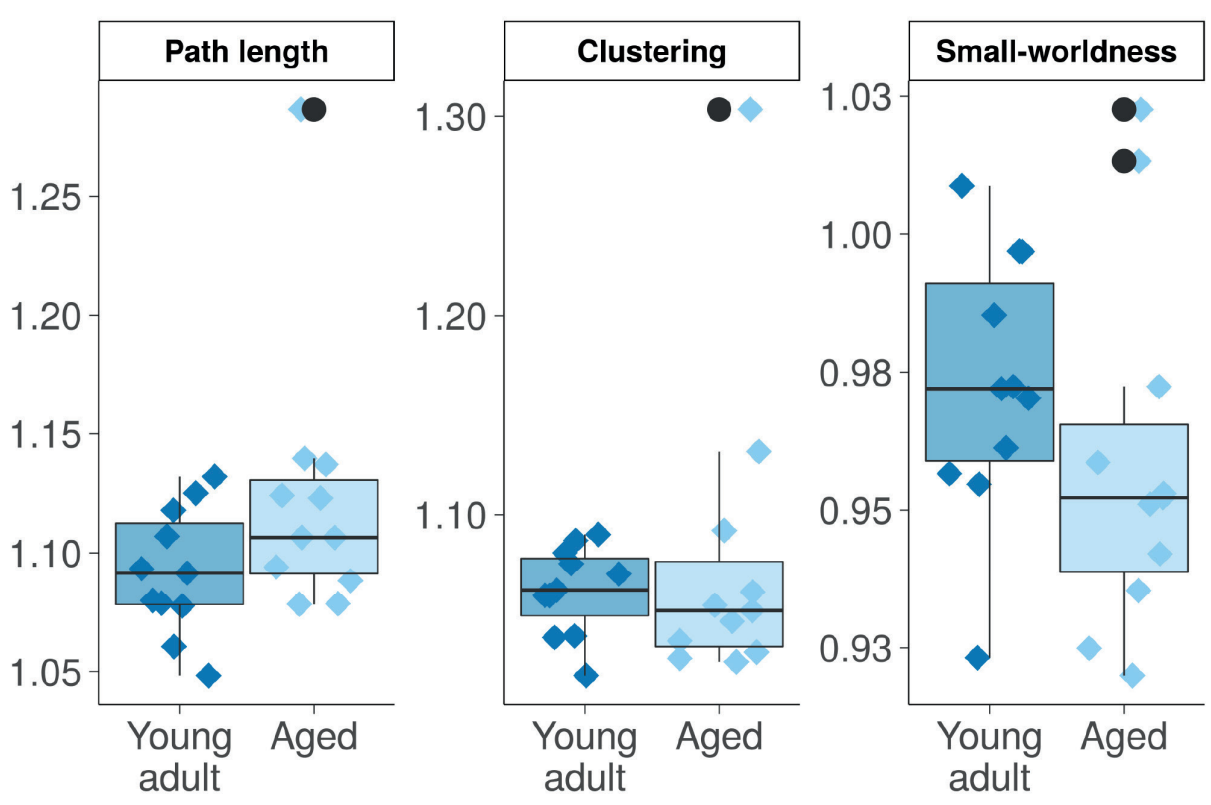

Figure S2. Global network parameters for whole-brain resting-state functional networks normalized by the maximum value in young adult and aged rats. Path length, clustering coefficient and smallworldness in resting-state fMRI-based whole-brain functional networks in young adult (dark blue) and aged rats (light blue). Individual values are given as diamonds with horizontal jitter for visualization purposes. Boxplots show median and inter-quartile range (IQR), whiskers representing 1.5 times the IQR, and black dots representing outliers. Outliers are determined as values exceeding 1.5 times the IQR above the upper and below the lower quartile. Clustering = Clustering coefficient. 

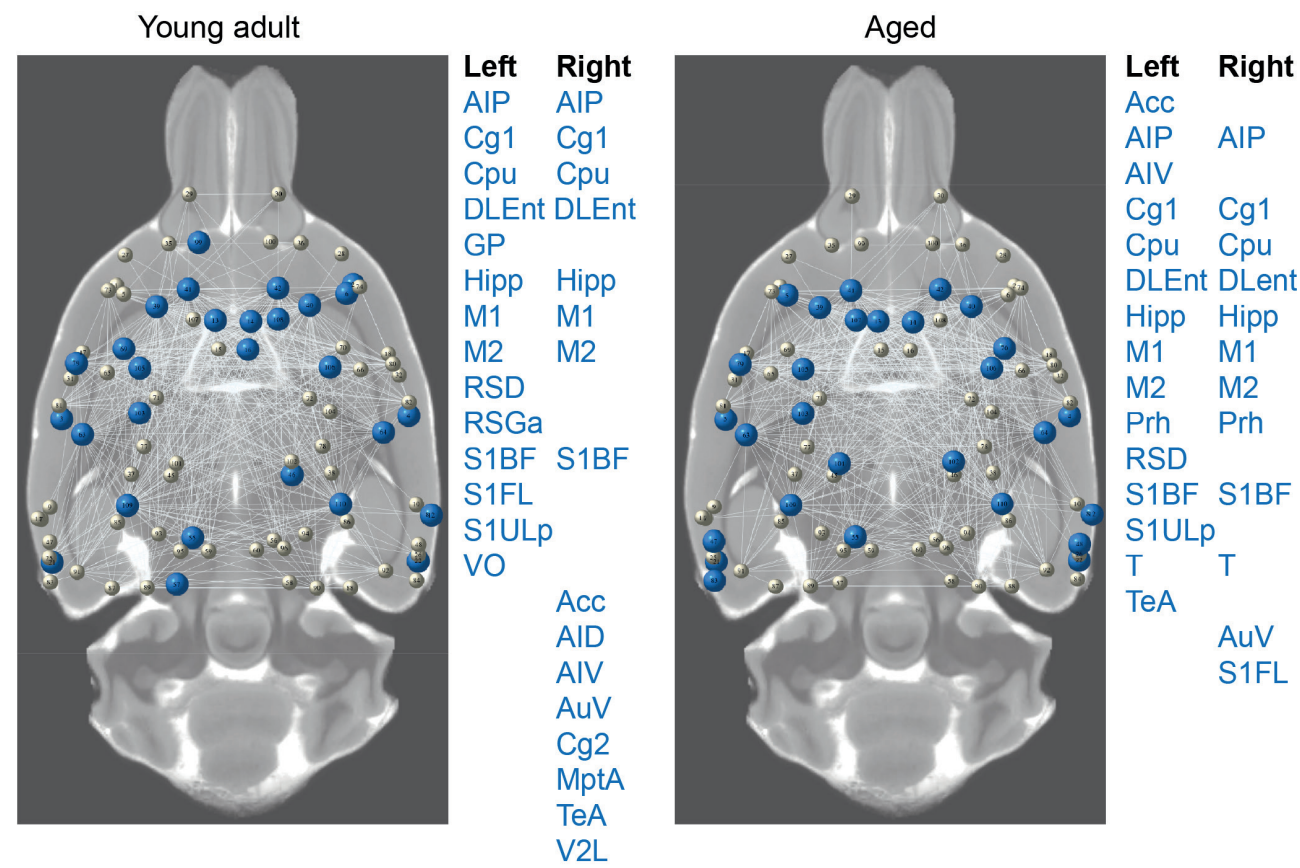

Figure S3. Hub regions in functional whole-brain networks normalized by the maximum value in young adult and aged rats. Hub regions in resting-state functional networks, overlaid on an axial structural MR image of a rat brain slice, are displayed in the 3D network as large blue nodes, whereas the other (non-hub) regions are presented as small white nodes. Networks are shown for young adult (left) and aged rats (right). Hub regions are listed right from the maps. 


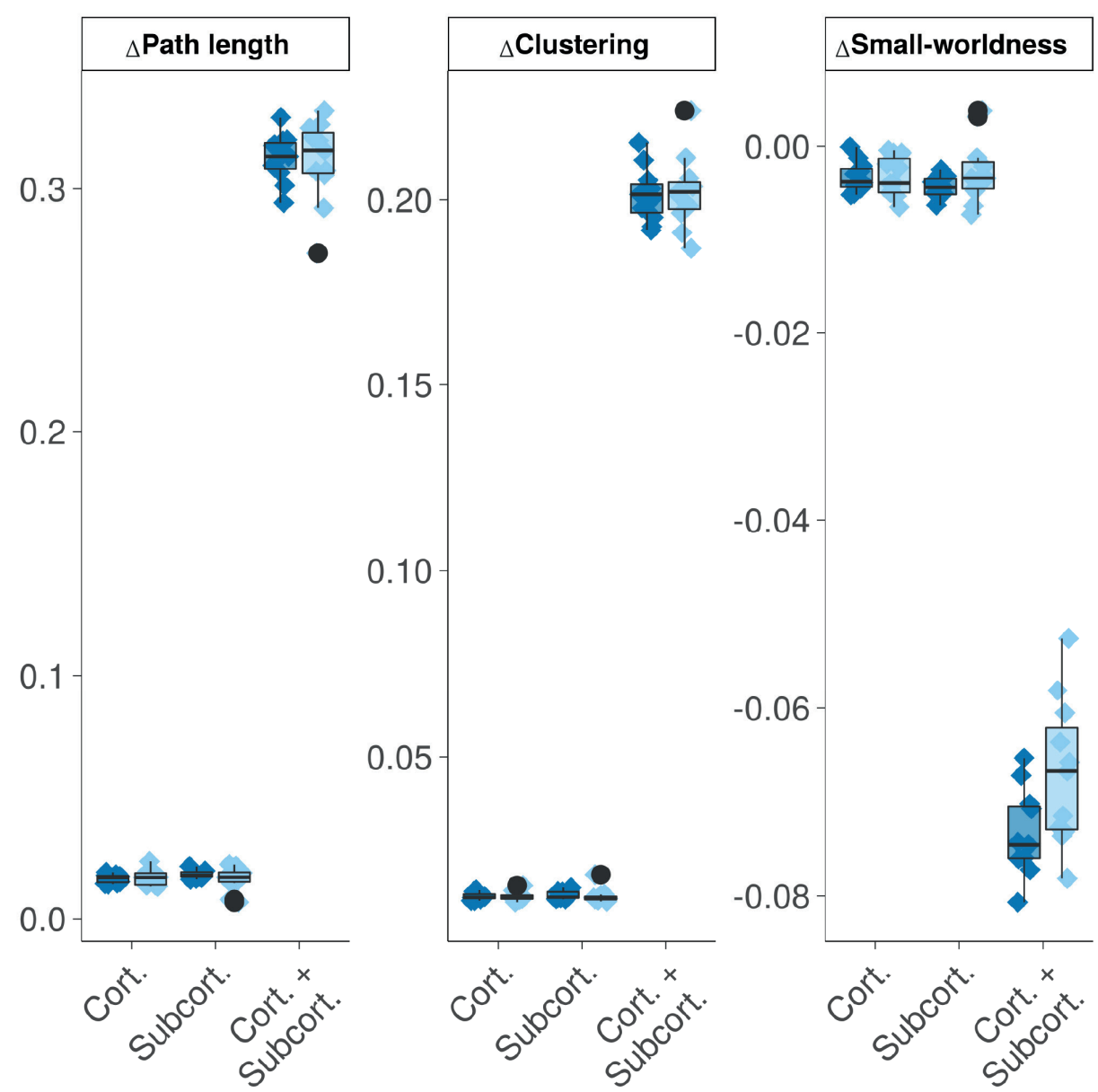

Figure S4. Effects of different stroke lesion simulations on global functional network measures normalized by the maximum value in young adult and old rats. Changes in path length, clustering and small-worldness after stroke lesion simulation, calculated as metric delta values between the healthy control network and the network after stroke simulation, for resting-state fMRI-based functional networks. Delta-scores were determined for different stroke lesion simulations, i.e. cort: cortical (photothrombosis), subcort: subcortical (short transient MCAO) and cort + subcort: cortical plus subcortical (long transient or permanent MCAO), for young adult rats (dark blue) and aged rats (light blue). Individual values are given as diamonds with horizontal jitter for visualization purposes. Boxplots show median and inter-quartile range (IQR), whiskers representing 1.5 times the IQR, and dots representing outliers. Outliers are defined as values exceeding 1.5 times the IQR above the upper and below the lower quartile. Clustering = Clustering coefficient. 
A) Small cortical lesion
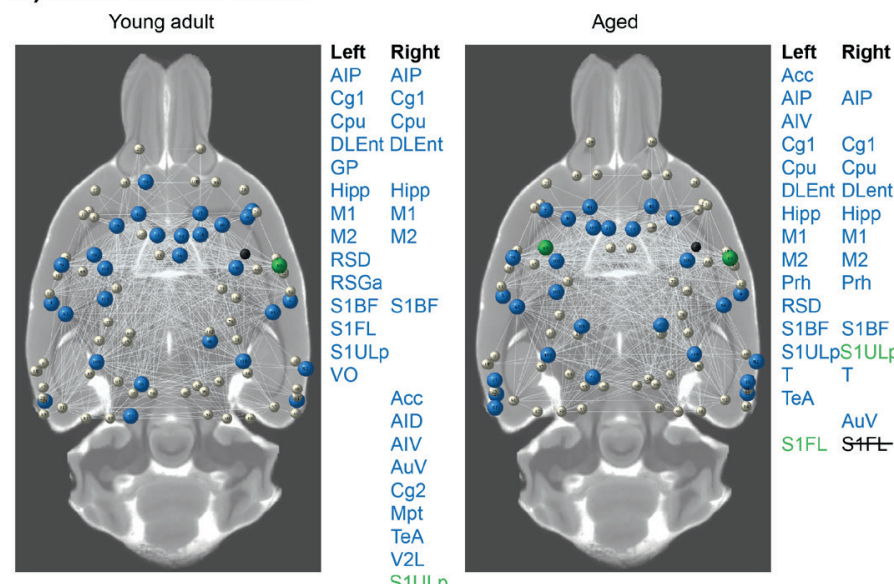

\section{B) Subcortical lesion}

Young adult
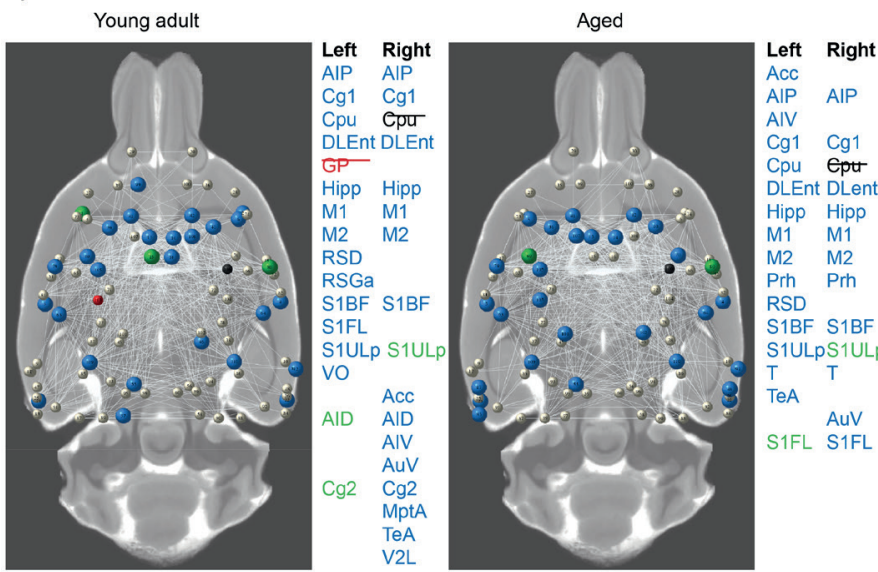

C) Large cortical and subcortical lesion
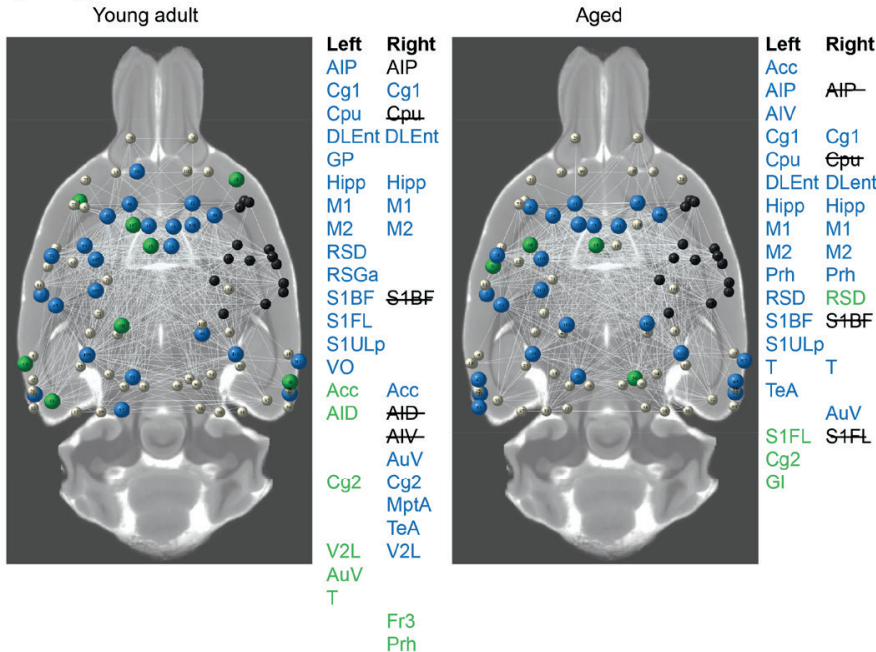

Figure S5. Hub shifts in functional whole-brain networks normalized by the maximum value after unilateral stroke lesion simulations in young adult and aged rat brain. Hub shifts are displayed after unilateral (right-sided) stroke lesion simulation in (A) a small cortical area, (B) a subcortical area and (C) a large cortical and subcortical area. Hub nodes, overlaid on an axial structural MR image of a rat brain slice, are displayed in the 3D network as large nodes, whereas other (non-hub) regions are represented as small (white) nodes. Regions with maintained hub status after stroke lesion simulation are shown as large blue nodes, whereas regions that acquired a hub status are presented as large green nodes. Regions that lost hub status are presented as small red nodes. Lesioned nodes are presented in dark-grey color. Networks are shown for young adult (left) and aged rats (right). Hub regions are listed right from the maps. Regions with acquired hub status are shown in green, and regions with lost hub status are crossed-out in red or black (lesioned nodes). 


\section{A) Global structural network}

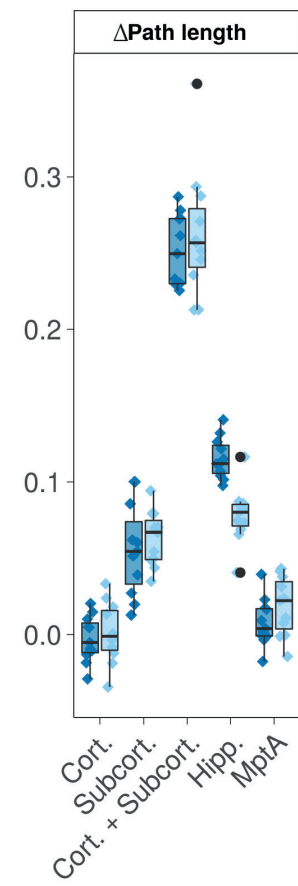

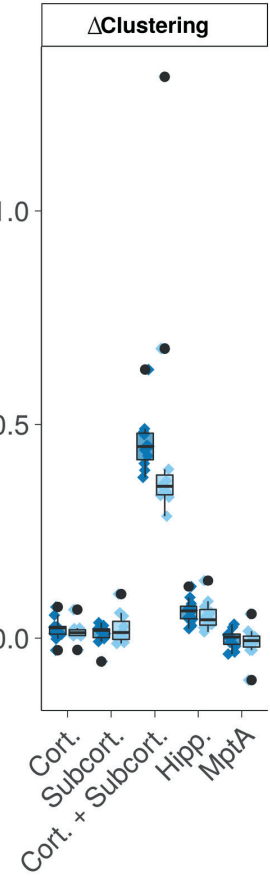

B) Global functional network

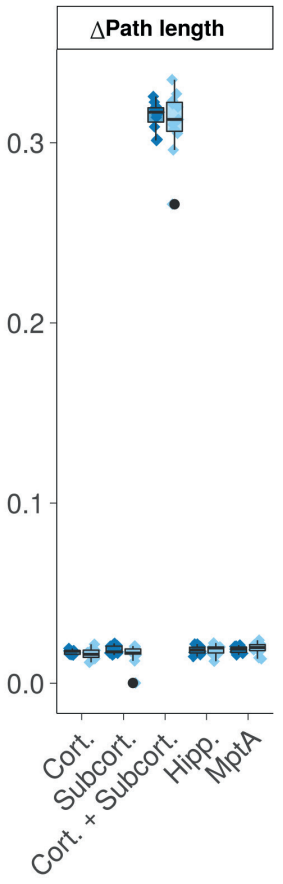

Figure S6: Effects of different stroke lesion simulations on global structural and functional network measures in young adult and old rats. Changes in path length, clustering and small-worldness after stroke lesion simulation, calculated as metric delta values between the healthy control network and the network after stroke simulation, for (A) diffusion-based structural networks and (B) restingstate fMRI-based functional networks. Delta-scores were determined for different stroke lesion simulations, i.e. cort: cortical (photothrombosis), subcort: subcortical (short transient MCAO), cort + subcort: cortical plus subcortical (long transient or permanent MCAO), and for specific lesions in the hippocampus (Hipp) or medial parietal association cortex (MptA), for young adult rats (dark blue) and aged rats (light blue). Individual values are shown as diamonds with horizontal jitter for visualization purposes. Boxplots show median and inter-quartile range (IQR), whiskers representing 1.5 times the IQR, and dots representing outliers. Outliers are defined as values exceeding 1.5 times the IQR above the upper and below the lower quartile. 


\section{CHAPTER 6}

A

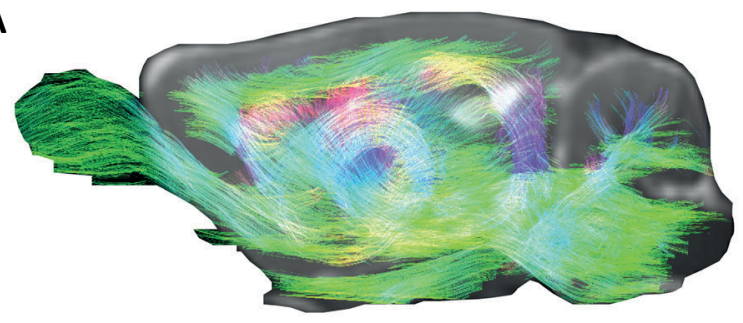

B

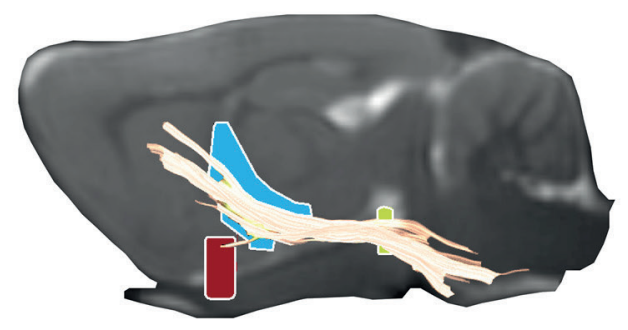

C

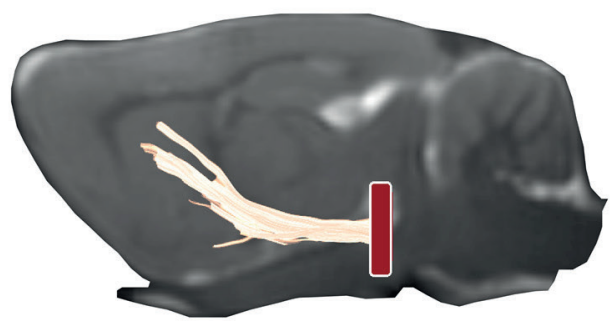

D

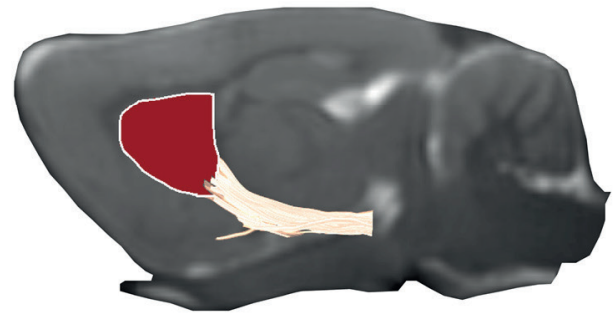

E

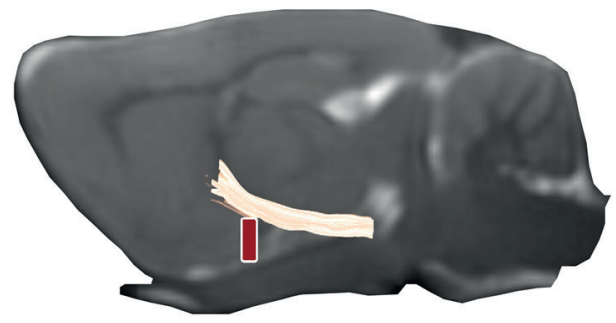

Figure S1. Schematic representation of the CST segmentation pipeline. (A) Whole-brain tractogram projected on an anatomical image of the rat brain (sagittal plane). The colors indicate the direction of the streamlines (blue $=$ inferiorsuperior; red = mediallateral; green = anteriorposterior). (B) From the whole-brain tractogram all streamlines were selected that passed through the brainstem (green rectangle) and internal capsule (blue region), but not the optic tract (red rectangle). (C) Subsequently, streamlines were cut off at the level of the brainstem (red rectangle). (D) Parts of streamlines that entered the striatum (red region) were masked out. (E) Finally, spurious tracts were removed through manually drawn masks (red rectangle). 


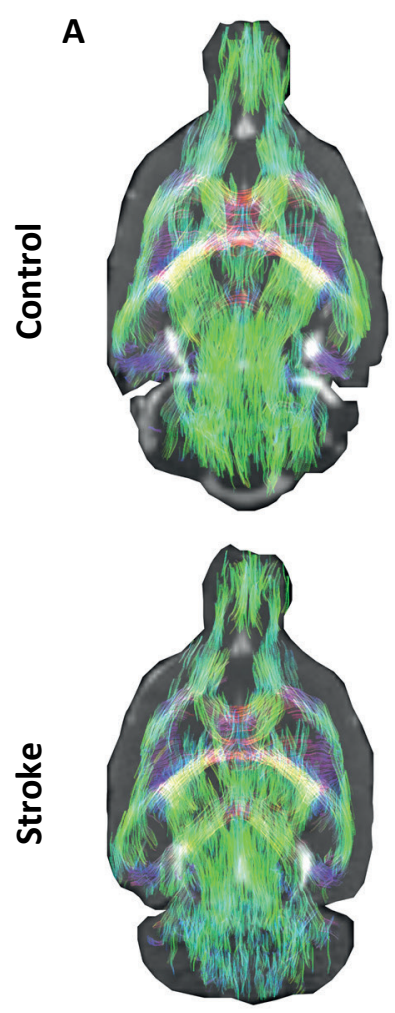

B
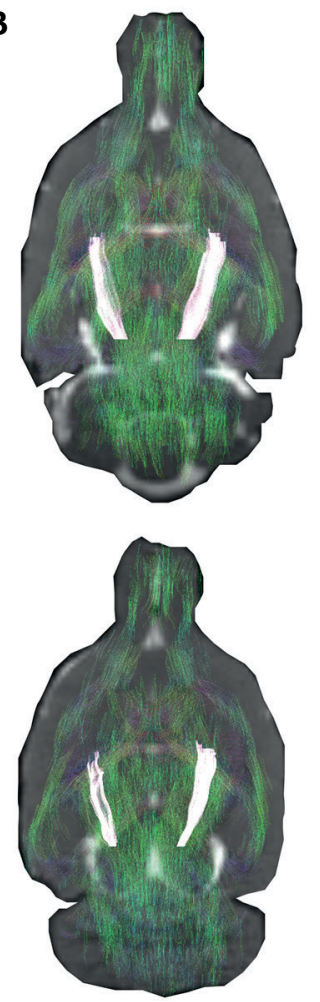

C
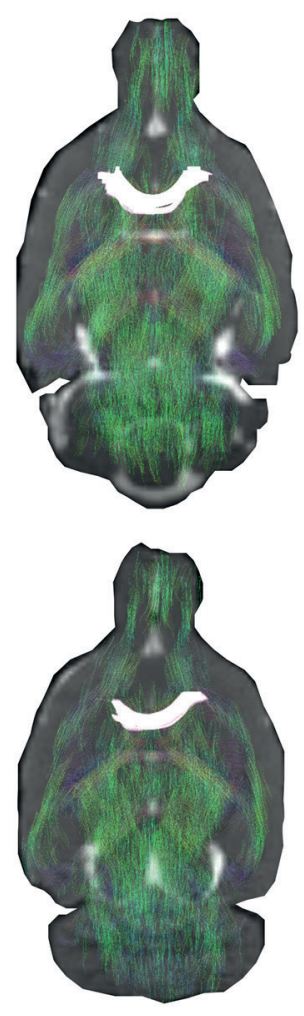

Figure S2. Diffusion MRI-based tractography and subsequent segmentations. (A) Whole-brain tractograms and (B) representative segmentations (white) of the bilateral CST and (C) transcallosal tracts of a control rat (top) and stroke rat (bottom) projected on an anatomical image of the rat brain (sagittal plane). 


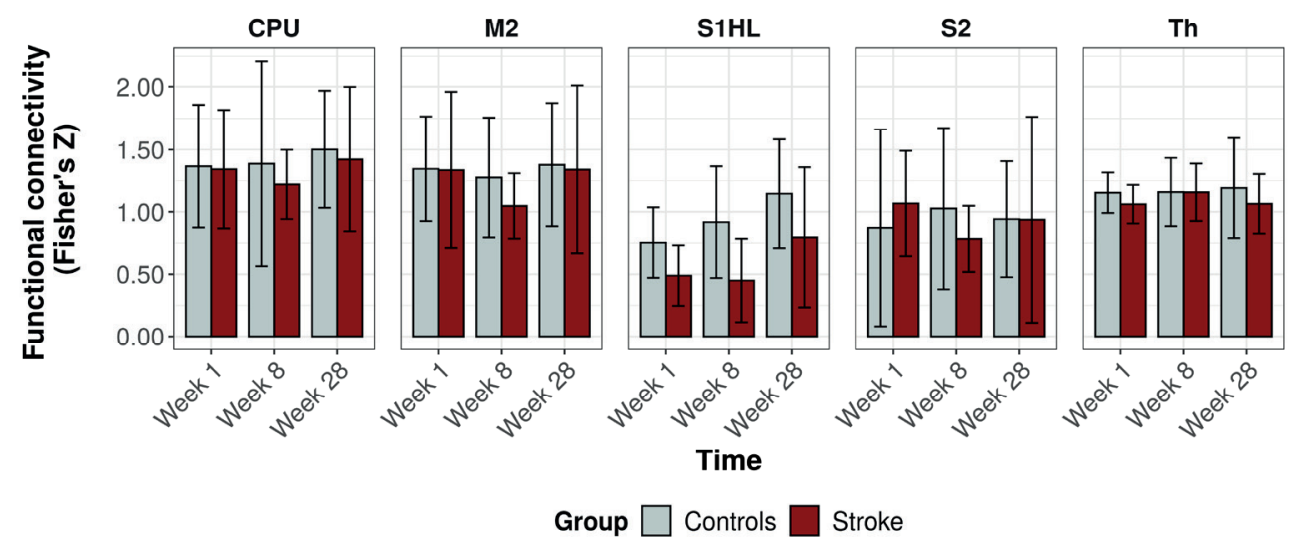

Figure S3. Interhemispheric functional connectivity over time for different sensorimotor regions. Functional connectivity as mean ( $\pm S D$ ) Fisher's $Z$ for the caudate putamen (CPU), secondary motor cortex (M2), hindlimb regions of the primary somatosensory cortex (S1FL), secondary somatosensory cortex (S2) and thalamus (Th) at 1 week, 8 weeks and 28 weeks for both controls (grey) and stroke rats $(r e d)$. 



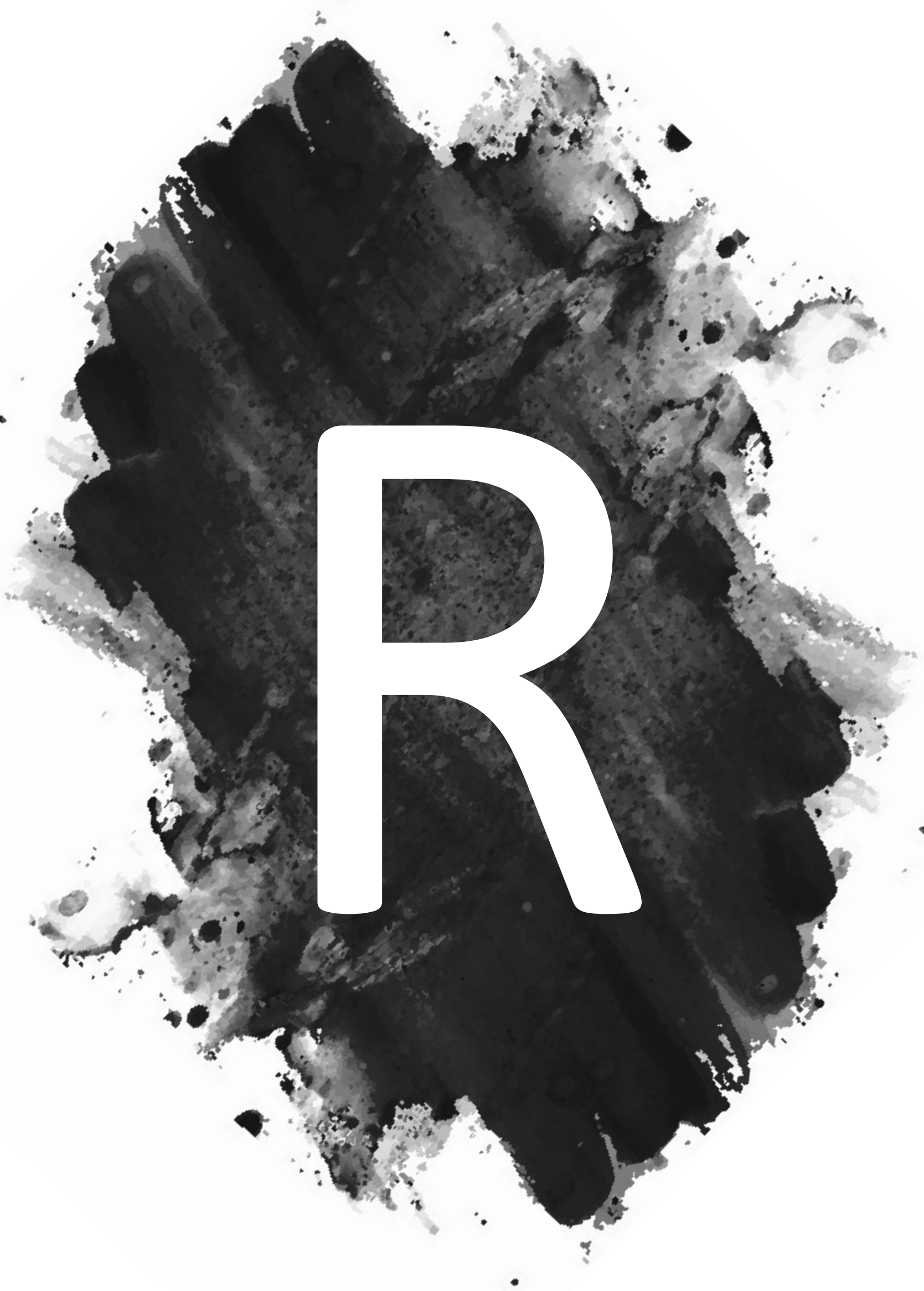


References 



\section{REFERENCES}

Abe, O., Aoki, S., Hayashi, N., Yamada, H., Kunimatsu, A., Mori, H., Yoshikawa, T., Okubo, T., \& Ohtomo, K. (2002). Normal aging in the central nervous system: Quantitative MR diffusion-tensor analysis. Neurobiology of Aging, 23(3), 433-441.

Achard, S., \& Bullmore, E. (2007). Efficiency and cost of economical brain functional networks. PLoS Computational Biology, 3(2), 0174-0183.

Adachi, Y., Osada, T., Sporns, O., Watanabe, T., Matsui, T., Miyamoto, K., \& Miyashita, Y. (2012). Functional connectivity between anatomically unconnected areas is shaped by collective networklevel effects in the macaque cortex. Cerebral Cortex, 22(7), 1586-1592.

Aerts, H., Fias, W., Caeyenberghs, K., \& Marinazzo, D. (2016). Brain networks under attack: Robustness properties and the impact of lesions. Brain, 139(12), 3063-3083.

Agcaoglu, O., Miller, R., Mayer, A. ., Hugdahl, K., \& Calhoun, V. . (2015). Lateralization of resting state networks and relationship to age and gender. Neurolmage, 104, 310-325.

Alexander-Bloch, A. F., Gogtay, N., Meunier, D., Birn, R., Clasen, L., Lalonde, F., Lenroot, R., Giedd, J., \& Bullmore, E. T. (2010). Disrupted modularity and local connectivity of brain functional networks in childhood-onset schizophrenia. Frontiers in Systems Neuroscience, 4, 1-16.

Alexander, D. C., Dyrby, T. B., Nilsson, M., \& Zhang, H. (2017). Imaging brain microstructure with diffusion MRI: Practicality and applications. NMR in Biomedicine, 32:e3841, 1-26.

Allred, R. P., Kim, S. Y., \& Jones, T. A. (2014). Use it and/or lose it-experience effects on brain remodeling across time after stroke. Frontiers in Human Neuroscience, 8, 1-8.

Alstott, J., Breakspear, M., Hagmann, P., Cammoun, L., \& Sporns, O. (2009). Modeling the impact of lesions in the human brain. PLoS Computational Biology, 5(6), e1000408, 1-12.

Altman, D. G., \& Bland, J. M. (2011). How to obtain the P value from a confidence interval. British Medical Journal (Clinical Research Ed.), 343, 1-2.

Andersson, J. L. R., Jenkinson, M., \& Smith, S. (2007). Non-linear registration, aka spatial normalisation. FMRIB Technial Report TR07JA2. In Oxford Centre for Functional Magnetic Resonance Imaging of the Brain, Department of Clinical Neurology, Oxford University, Oxford, UK (Issue June).

Andersson, J. L. R., \& Sotiropoulos, S. N. (2016). An integrated approach to correction for offresonance effects and subject movement in diffusion MR imaging. Neurolmage, 125, 1063-1078.

Andrews-Hanna, J. R., Snyder, A. Z., Vincent, J. L., Lustig, C., Head, D., Raichle, M. E., \& Buckner, R. L. (2007). Disruption of large-scale brain systems in advanced aging. Neuron, 56(5), 924-935.

Andriessen, T. M. J. C., Jacobs, B., \& Vos, P. E. (2010). Clinical characteristics and pathophysiological mechanisms of focal and diffuse traumatic brain injury. Journal of Cellular and Molecular Medicine, 14(10), 2381-2392.

Arenivas, A., Diaz-Arrastia, R., Spence, J., Cullum, C. M., Krishnan, K., Bosworth, C., Culver, C., Kennard, B., \& Marquez de la Plata, C. (2014). Three approaches to investigating functional compromise to the default mode network after traumatic axonal injury. Brain Imaging and Behavior, 8(3), 407-419. 
Assaf, Y., Alexander, D. C., Jones, D. K., Bizzi, A., Behrens, T. E. J., Clark, C. A., Cohen, Y., Dyrby, T. B., Huppi, P. S., Knoesche, T. R., LeBihan, D., Parker, G. J. M., Poupon, C., Anaby, D., Anwander, A., Bar, L., Barazany, D., Blumenfeld-Katzir, T., De-Santis, S., ... Zhou, F. L. (2013). The CONNECT project: Combining macro- and micro-structure. Neurolmage, 80, 273-282.

Assaf, Y., \& Basser, P. J. (2005). Composite hindered and restricted model of diffusion (CHARMED) MR imaging of the human brain. Neurolmage, $27,48-58$.

Assaf, Y., Johansen-Berg, H., \& Thiebaut de Schotten, M. (2017). The role of diffusion MRI in neuroscience. NMR in Biomedicine, 32:e3762, 1-16.

Avena-Koenigsberger, A., Misic, B., \& Sporns, O. (2017). Communication dynamics in complex brain networks. Nature Reviews Neuroscience, 19(1), 17-33.

Azadbakht, H., Parkes, L. M., Haroon, H. A., Augath, M., Logothetis, N. K., de Crespigny, A., D'Arceuil, H. E., \& Parker, G. J. M. (2015). Validation of high-resolution tractography against in Vivo tracing in the macaque visual cortex. Cerebral Cortex, 25(11), 4299-4309.

Bachmann, L. C., Lindau, N. T., Felder, P., \& Schwab, M. E. (2014). Sprouting of brainstem-spinal tracts in response to unilateral motor cortex stroke in mice. Journal of Neuroscience, 34(9), 3378-3389.

Bains, M., \& Hall, E. D. (2012). Antioxidant therapies in traumatic brain and spinal cord injury. Biochimica et Biophysica Acta, 1822(5), 675-684.

Balls, M., Goldberg, A., Fentem, J. H., Broadhead, C., Bursch, R., Festing, M., Frazier, J., Hendriksen, F., Jennings, M., van der Kamp, M., Morton, D., Rowan, A., Russell, C., Russell, W., Spielmann, H., Stephens, M., Stokes, W., Straughan, D., Yager, J., ... van Zutphen, B. (1995). The three Rs: the way forward. Alternatives to Laboratory Animals: ATLA, 23, 838-866.

Barrat, A., Barthélemy, M., Pastor-Satorras, R., \& Vespignani, A. (2004). The architecture of complex weighted networks. Proceedings of the National Academy of Sciences, 101(11), 3747-3752.

Barzó, P., Marmarou, A., Fatouros, P., Corwin, F., \& Dunbar, J. (1996). Magnetic resonance imagingmonitored acute blood-brain barrier changes in experimental traumatic brain injury. Journal of Neurosurgery, 85(6), 1113-1121.

Basser, P.J., Mattiello, J., \& LeBihan, D. (1994). Estimation of the effective self-diffusion tensor from the NMR spin echo. In Journal of Magnetic Resonance, Series B, 103(3), 247-254.

Basser, P. J., Mattiello, J., \& LeBihan, D. (1994). MR diffusion tensor spectroscopy and imaging. Biophysical Journal, 66(1), 259-267.

Basser, P. J., Pajevic, S., Pierpaoli, C., Duda, J., \& Aldroubi, A. (2000). In vivo fiber tractography using DT-MRI data. Magnetic Resonance in Medicine, 44, 625-632.

Bassett, D. S., \& Bullmore, E. T. (2009). Human brain networks in health and disease. Current Opinion in Neurology, 22(4), 340-347.

Bassett, D. S., \& Sporns, O. (2017). Network Neuroscience. Nature Neuroscience, 20(3), 353-364.

Bassett, D. S., Zurn, P., \& Gold, J. I. (2018). On the nature and use of models in network neuroscience. Nature Reviews Neuroscience, 19(9), 566-578. 
Bastiani, M., Shah, N. J., Goebel, R., \& Roebroeck, A. (2012). Human cortical connectome reconstruction from diffusion weighted MRI: The effect of tractography algorithm. Neurolmage, 62, 1732-1749.

Bauer, A. Q., Kraft, A. W., Baxter, G. A., Wright, P. W., Reisman, M. D., Bice, A. R., Park, J. J., Bruchas, M. R., Snyder, A. Z., Lee, J.-M., \& Culver, J. P. (2017). Effective connectivity measured using optogenetically evoked hemodynamic signals exhibits topography distinct from resting state functional connectivity in the mouse. Cerebral Cortex, February, 1-17.

Bauer, A. Q., Kraft, A. W., Wright, P. W., Snyder, A. Z., Lee, J. M., \& Culver, J. P. (2014). Optical imaging of disrupted functional connectivity following ischemic stroke in mice. Neurolmage, 99, 388-401.

Bazarian, J. J., Zhong, J., Blyth, B., Zhu, T., Kavcic, V., \& Peterson, D. (2007). Diffusion tensor imaging detects clinically important axonal damage after mild traumatic brain injury: A pilot study. Journal of Neurotrauma, 24(9), 1447-1459.

Beaulieu, C. (2002). The basis of anisotropic water diffusion in the nervous system - A technical review. NMR in Biomedicine, 15(7-8), 435-455.

Beaumont, A., Marmarou, A., Hayasaki, K., Barzo, P., Fatouros, P., Corwin, F., Marmarou, C., \& Dunbar, J. (2000). The permissive nature of blood brain barrier (BBB) opening in edema formation following traumatic brain injury. Acta Neurochirurgica. Supplement, 76, 125-129.

Belanger, H. G., Vanderploeg, R. D., Curtiss, G., \& Warden, D. L. (2007). Recent neuroimaging techniques in mild traumatic brain injury. Journal of Neuropsychiatry, 19(1), 5-20.

Benjamini, Y., \& Hochberg, Y. (1995). Controlling the false discovery rate - A practical and powerful approach to multiple testing. Journal of the Royal Statistical Society Series B (Methodological), 57(1), 289-300.

Bergonzi, K. M., Bauer, A. Q., Wright, P. W., \& Culver, J. P. (2015). Mapping functional connectivity using cerebral blood flow in the mouse brain. Journal of Cerebral Blood Flow and Metabolism, 35(3), 367-370.

Betzel, R. F., Avena-Koenigsberger, A., Goñi, J., He, Y., de Reus, M. A., Griffa, A., Vértes, P. E., Mišic, B., Thiran, J.-P., Hagmann, P., van den Heuvel, M., Zuo, X.-N., Bullmore, E. T., \& Sporns, O. (2015). Generative models of the human connectome. Neurolmage, 124, 1054-1064.

Betzel, R. F., Byrge, L., He, Y., Goni, J., Zuo, X. N., \& Sporns, O. (2014). Changes in structural and functional connectivity among resting-state networks across the human lifespan. Neurolmage, 102, 345-357.

Biernaskie, J., \& Corbett, D. (2001). Enriched rehabilitative training promotes improved forelimb motor function and enhanced dendritic growth after focal ischemic injury. The Journal of Neuroscience, 21(14), 5272-5280.

Biswal, B. B., Zerrin Yetkin, F., Haughton, V. M., \& Hyde, J. S. (1995). Functional connectivity in the motor cortex of resting human brain using echo-planar MRI. Magnetic Resonance in Medicine, 34(4), 537-541.

Bland, J. M., \& Altman, D. G. (2015). Statistics notes: Bootstrap resampling methods. British Medical Journal, 350:h2622, 2-3. 
Blockx, I., van Camp, N., Verhoye, M., Boisgard, R., Dubois, A., Jego, B., Jonckers, E., Raber, K., Siquier, K., Kuhnast, B., Dollé, F., Nguyen, H. P., Von Hörsten, S., Tavitian, B., \& van der Linden, A. (2011). Genotype specific age related changes in a transgenic rat model of Huntington's disease. Neurolmage, 58, 1006-1016.

Boersma, M., Smit, D. J. A., Boomsma, D. I., De Geus, E. J. C., Delemarre-van de Waal, H. A., \& Stam, C. J. (2013). Growing trees in child brains: graph theoretical analysis of electroencephalographyderived minimum spanning tree in 5- and 7-year-old children reflects brain maturation. Brain Connectivity, 3(1), 50-60.

Boonzaier, J., van Tilborg, G. A. F., Neggers, S. F. W., \& Dijkhuizen, R. M. (2018). Noninvasive brain stimulation to enhance functional recovery after stroke: Studies in animal models. Neurorehabilitation and Neural Repair, 32(11), 927-940.

Borg, J., Holm, L., Cassidy, J. D., Peloso, P. M., Carroll, L. J., von Holst, H., \& Ericson, K. (2004). Diagnostic procedures in mild traumatic brain injury: results of the WHO Collaborating Centre Task Force on Mild Traumatic Brain Injury. Journal of Rehabilitation Medicine, 43 Suppl, 61-75.

Bounova, G., \& de Weck, O. (2012). Overview of metrics and their correlation patterns for multiplemetric topology analysis on heterogeneous graph ensembles. Physical Review E, 85(1), 16117.

Braeckman, K., Descamps, B., Pieters, L., Vral, A., Caeyenberghs, K., \& Vanhove, C. (2019). Dynamic changes in hippocampal diffusion and kurtosis metrics following experimental mTBI correlate with glial reactivity. Neurolmage: Clinical, 21, 101669.

Brandes, U. (2011). A faster algorithm for betweenness centrality. Journal of Mathematical Sociology, 25(2), 163-177.

Broyd, S. J., Demanuele, C., Debener, S., Helps, S. K., James, C. J., \& Sonuga-Barke, E. J. S. (2009). Default-mode brain dysfunction in mental disorders: A systematic review. Neuroscience and Biobehavioral Reviews, 33(3), 279-296.

Bullmore, E., Barnes, A., Bassett, D. S., Fornito, A., Kitzbichler, M., Meunier, D., \& Suckling, J. (2009). Generic aspects of complexity in brain imaging data and other biological systems. Neurolmage, 47, 1125-1134.

Bullmore, E., \& Sporns, O. (2009). Complex brain networks: graph theoretical analysis of structural and functional systems. Nature Reviews Neuroscience, 10(3), 186-198.

Bullmore, E., \& Sporns, O. (2012). The economy of brain network organization. Nature Reviews Neuroscience, 13(5), 336-349.

Burke, S. N., \& Barnes, C. A. (2006). Neural plasticity in the ageing brain. Nature Reviews Neuroscience, 7(1), 30-40.

Cai, J., Ji, Q., Xin, R., Zhang, D., Na, X., Peng, R., \& Li, K. (2016). Contralesional cortical structural reorganization contributes to motor recovery after sub-cortical stroke: a longitudinal voxel-based morphometry study. Frontiers in Human Neuroscience, 10(8), 393.

Caimo, A., \& Friel, N. (2011). Bayesian inference for exponential random graph models. Social Networks, 33(1), 41-55. 
Caimo, A., \& Friel, N. (2014). Bergm: Bayesian Exponential Random Graphs in R. Technical Report, arXiv:1201.2770, 1-27.

Caimo, A., \& Mira, A. (2014). Efficient computational strategies for doubly intractable problems with applications to Bayesian social networks. Statistics and Computing, 25, 113-125.

Calabrese, E., Badea, A., Cofer, G., Qi, Y., \& Johnson, G. A. (2015). A diffusion MRI tractography connectome of the mouse brain and comparison with neuronal tracer data. Cerebral Cortex, 1-10.

Calabrese, E., \& Johnson, G. A. (2013). Diffusion tensor magnetic resonance histology reveals microstructural changes in the developing rat brain. Neurolmage, 79, 329-339.

Cao, M., Wang, J. H., Dai, Z. J., Cao, X. Y., Jiang, L. L., Fan, F. M., Song, X. W., Xia, M. R., Shu, N., Dong, Q., Milham, M. P., Castellanos, F. X., Zuo, X. N., \& He, Y. (2014). Topological organization of the human brain functional connectome across the lifespan. Developmental Cognitive Neuroscience, $7(16), 76-$ 93.

Carmichael, S. T., \& Chesselet, M.-F. (2002). Synchronous neuronal activity is a signal for axonal sprouting after cortical lesions in the adult. Journal of Neuroscience, 22(14), 6062-6070.

Carrera, E., \& Tononi, G. (2014). Diaschisis: Past, present, future. Brain, 137(9), 2408-2422.

Carter, A. R., Astafiev, S. V., Lang, C. E., Connor, L. T., Rengachary, J., Strube, M. J., Pope, D. L. W., Shulman, G. L., \& Corbetta, M. (2010). Resting inter-hemispheric fMRI connectivity predicts performance after stroke. Annals of Neurology, 67(3), 365-375.

Carter, A. R., Patel, K. R., Astafiev, S. V., Snyder, A. Z., Rengachary, J., Strube, M. J., Pope, A., Shimony, J. S., Lang, C. E., Shulman, G. L., \& Corbetta, M. (2012). Upstream dysfunction of somatomotor functional connectivity after corticospinal damage in stroke. Neurorehabilitation and Neural Repair, 26(1), 7-19.

Carter, A. R., Shulman, G. L., \& Corbetta, M. (2012). Why use a connectivity-based approach to study stroke and recovery of function? Neurolmage, 62, 2271-2280.

Castellanos, N. P., Paúl, N., Ordóñ Ez, V. E., Demuynck, O., Bajo, R., Campo, P., Bilbao, A., Ortiz, T., DelPozo, F., \& Maestú, F. (2010). Reorganization of functional connectivity as a correlate of cognitive recovery in acquired brain injury. Brain, 133(8), 2365-2381.

Chen, H., Liu, T., Zhao, Y., Zhang, T., Li, Y., Li, M., Zhang, H., Kuang, H., Guo, L., Tsien, J. Z., \& Liu, T. (2015). Optimization of large-scale mouse brain connectome via joint evaluation of DTI and neuron tracing data. Neurolmage, 115, 202-213.

Cheng, B., Dietzmann, P., Schulz, R., Boenstrup, M., Krawinkel, L., Fiehler, J., Gerloff, C., \& Thomalla, G. (2019). Cortical atrophy and transcallosal diaschisis following isolated subcortical stroke. Journal of Cerebral Blood Flow and Metabolism, 40(3), 611-621.

Cheng, B., Schlemm, E., Schulz, R., \& Boenstrup, M. (2019). Altered topology of large-scale structural brain networks in chronic stroke. Brain Communications, 1-13.

Chia-Koh, L., Pei-Tang, F., Chen, H. I., Yung-Hsu, C., Ching-Hsieh, L., \& Tseng, W. Y. I. (2018). Impaired callosal motor fiber integrity and upper extremity motor impairment are associated with stroke lesion location. Neurorehabilitation and Neural Repair, 32(6-7), 602-612. 
Chiang, A. S., Lin, C. Y., Chuang, C. C., Chang, H. M., Hsieh, C. H., Yeh, C. W., Shih, C. T., Wu, J. J., Wang, G. T., Chen, Y. C., Wu, C. C., Chen, G. Y., Ching, Y. T., Lee, P. C., Lin, C. Y., Lin, H. H., Wu, C. C., Hsu, H. W., Huang, Y. A., ... Hwang, J. K. (2011). Three-dimensional reconstruction of brain-wide wiring networks in drosophila at single-cell resolution. Current Biology, 21(1), 1-11.

Christiaens, D., Maes, F., Sunaert, S., \& Suetens, P. (2015). Imposing label priors in global tractography can resolve crossing fibre ambiguities. Proc. ISMRM, 23(1031).

Christiaens, D., Reisert, M., Dhollander, T., Maes, F., Sunaert, S., \& Suetens, P. (2014). Atlas-guided global tractography: imposing a prior on the local track orientation. In L. O'Donnell, G. Nedjati-Gilani, Y. Rathi, M. Reisert, \& T. Schneider (Eds.), Computational Diffusion MRI (pp. 203-213).

Christiaens, D., Reisert, M., Dhollander, T., Sunaert, S., Suetens, P., \& Maes, F. (2015). Global tractography of multi-shell diffusion-weighted imaging data using a multi-tissue model. Neurolmage, 123, 89-101.

Chu, C. Y., Huang, J. P., Sun, C. Y., Zhang, Y. L., Liu, W. Y., \& Zhu, Y. M. (2015). Estimating intravoxel fiber architecture using constrained compressed sensing combined with multitensor adaptive smoothing. International Journal of Imaging Systems and Technology, 25(4), 285-296.

Collier, T. J., \& Kordower, J. H. (2012). Ageing as a primary risk factor for Parkinson's disease: evidence from studies of non-human primates. Nature Reviews Neuroscience, 12(6), 359-366.

Cox, R. W. (1996). AFNI: Software for analysis and visualization of functional magnetic resonance neuroimages. Computers and Biomedial Research, 29, 162-173.

Cramer, S. C. (2008). Repairing the human brain after stroke: I. Mechanisms of spontaneous recovery. Annals of Neurology, 63(3), 272-287.

Crofts, J. J., Higham, D. J., Bosnell, R., Jbabdi, S., Matthews, P. M., Behrens, T. E. J., \& Johansen-Berg, H. (2011). Network analysis detects changes in the contralesional hemisphere following stroke. Neurolmage, 54, 161-169.

Crossley, N. A., Mechelli, A., Scott, J., Carletti, F., Fox, P. T., Mcguire, P., \& Bullmore, E. T. (2014). The hubs of the human connectome are generally implicated in the anatomy of brain disorders. Brain, 137(8), 2382-2395.

Dacosta-Aguayo, R., Graña, M., Fernández-Andújar, M., López-Cancio, E., Cáceres, C., Bargalló, N., Barrios, M., Clemente, I., Monserrat, P. T., Sas, M. A., Dávalos, A., Auer, T., \& Mataró, M. (2014). Structural integrity of the contralesional hemisphere predicts cognitive impairment in ischemic stroke at three months. PLOS ONE, 9(1), 1-11.

Daducci, A., Palú, A. D., Descoteaux, M., \& Thiran, J. P. (2016). Microstructure informed tractography: Pitfalls and open challenges. Frontiers in Neuroscience, 10(6), 1-13.

Damoiseaux, J. S. (2017). Effects of aging on functional and structural brain connectivity. Neurolmage, $160,32-40$.

Dancause, N. (2005). Extensive cortical rewiring after brain injury. Journal of Neuroscience, 25(44), 10167-10179. 
Dauguet, J., Peled, S., Berezovskii, V., Delzescaux, T., Warfield, S. K., Born, R., \& Westin, C.-F. (2007). Comparison of fiber tracts derived from in-vivo DTI tractography with 3D histological neural tract tracer reconstruction on a macaque brain. Neurolmage, 37, 530-538.

De Haan, W., Mott, K., van Straaten, E. C. W., Scheltens, P., \& Stam, C. J. (2012). Activity dependent degeneration explains hub vulnerability in Alzheimer's disease. PLoS Computational Biology, 8(8).

Delettre, C., Messé, A., Dell, L.-A., Foubet, O., Heuer, K., Larrat, B., Meriaux, S., Mangin, J.-F., Reillo, I., de Juan Romero, C., Borrell, V., Toro, R., \& Hilgetag, C. C. (2019). Comparison between diffusion MRI tractography and histological tract-tracing of cortico-cortical structural connectivity in the ferret brain. Network Neuroscience, 3(4), 1038-1050.

Dell'Acqua, F., Bodi, I., Slater, D., Catani, M., \& Modo, M. (2013). MR diffusion histology and microtractography reveal mesoscale features of the human cerebellum. Cerebellum, 12(6), 923-931.

Dennis, E. L., Jahanshad, N., McMahon, K. L., de Zubicaray, G. I., Martin, N. G., Hickie, I. B., Toga, A. W., Wright, M. J., \& Thompson, P. M. (2013). Development of brain structural connectivity between ages 12 and 30: A 4-Tesla diffusion imaging study in 439 adolescents and adults. Neurolmage, 64, 671684.

Descoteaux, M., Deriche, R., \& Anwander, A. (2007). Deterministic and probabilistic q-ball tractography: from diffusion to sharp fiber distributions. Technical Reports 6273, INRIA Sophia Antipolis.

Desikan, R. S., Ségonne, F., Fischl, B., Quinn, B. T., Dickerson, B. C., Blacker, D., Buckner, R. L., Dale, A. M., Maguire, R. P., Hyman, B. T., Albert, M. S., \& Killiany, R. J. (2006). An automated labeling system for subdividing the human cerebral cortex on MRI scans into gyral based regions of interest. Neurolmage, 31, 968-980.

Desmarais, B. A., \& Cranmer, S. J. (2012). Statistical inference for valued-edge networks: The generalized exponential random graph model. PLOS ONE, 7(1), e30136.

Dijkhuizen, R. M., Ren, J., Mandeville, J. B., Wu, O., Ozdag, F. M., Moskowitz, M. A., Rosen, B. R., \& Finklestein, S. P. (2002). Functional magnetic resonance imaging of reorganization in rat brain after stroke. Proceedings of the National Academy of Sciences, 98(22), 12766-12771.

Dijkhuizen, R. M., \& Nicolay, K. (2003). Magnetic resonance imaging in experimental models of brain disorders. Journal of Cerebral Blood Flow and Metabolism, 23(12), 1383-1402.

Dijkhuizen, R. M., van der Marel, K., Otte, W. M., Hoff, E. I., van der Zijden, J. P., van der Toorn, A., \& van Meer, M. P. A. (2012). Functional MRI and diffusion tensor imaging of brain reorganization after experimental stroke. Translational Stroke Research, 3(1), 36-43.

Dijkhuizen, R. M., Zaharchuk, G., \& Otte, W. M. (2014). Assessment and modulation of resting-state neural networks after stroke. Current Opinion in Neurology, 27(6), 637-643.

Dijkhuizen, R. M., Sarabdjitsingh, R. A., Loi, M., \& Joe, M. (2017). Early life stress-induced alterations in rat brain structures measured with high resolution MRI. PLOS ONE, 12(9), 1-14.

Dijkstra, E. W. (1959). A note on two problems in connexion with graphs. Numerische Mathematik, 1(1), 269-271. 
Dirnagl, U. (2016). Thomas Willis lecture: Is translational stroke research broken, and if so, how can we fix it? Stroke, 47, 2148-2153.

Donahue, C. J., Sotiropoulos, S. N., Jbabdi, S., Hernandez-Fernandez, M., Behrens, T. E., Dyrby, T. B., Coalson, T., Kennedy, H., Knoblauch, K., van Essen, D. C., \& Glasser, M. F. (2016). Using diffusion tractography to predict cortical connection strength and distance: a quantitative comparison with tracers in the monkey. The Journal of Neuroscience, 36(25), 6758-6770.

Drakesmith, M., Caeyenberghs, K., Dutt, A., Lewis, G., David, A. S., \& Jones, D. K. (2015). Overcoming the effects of false positives and threshold bias in graph theoretical analyses of neuroimaging data. Neurolmage, 118, 313-333.

Duering, M., Righart, R., Wollenweber, F. A., Zietemann, V., Gesierich, B., \& Dichgans, M. (2015). Acute infarcts cause focal thinning in remote cortex via degeneration of connecting fiber tracts. Neurology, 84(16), 1685-1692.

Dyrby, T. B., Søgaard, L. V, Parker, G. J., Alexander, D. C., Lind, N. M., Baaré, W. F. C., Hay-Schmidt, A., Eriksen, N., Pakkenberg, B., Paulson, O. B., \& Jelsing, J. (2007). Validation of in vitro probabilistic tractography. Neurolmage, 37, 1267-1277.

Edlow, B. L., Copen, W. A., Izzy, S., Bakhadirov, K., van der Kouwe, A., Glenn, M. B., Greenberg, S. M., Greer, D. M., \& Wu, O. (2016). Diffusion tensor imaging in acute-to-subacute traumatic brain injury: A longitudinal analysis. BMC Neurology, 16(1), 1-11.

Engels, M. M., Stam, C. J., van der Flier, W. M., Scheltens, P., de Waal, H., \& van Straaten, E. C. (2015). Declining functional connectivity and changing hub locations in Alzheimer's disease: an EEG study. BMC Neurology, 15(1), 145.

Esposito, M. S., Capelli, P., \& Arber, S. (2014). Brainstem nucleus MdV mediates skilled forelimb motor tasks. Nature, 508(7496), 351-356.

Fagiolo, G. (2007). Clustering in complex directed networks. Physical Review, 76, 76:026107.

Feigin, V. L., Forouzanfar, M. H., Krishnamurthi, R., Mensah, G. A., Connor, M., Bennett, D. A., Moran, A. E., Sacco, R. L., Anderson, L., Truelsen, T., O'Donnell, M., Venketasubramanian, N., Barker-Collo, S., Lawes, C. M. M., Wang, W., Shinohara, Y., Witt, E., Ezzati, M., ... Naghavi, M. (2014). Global and regional burden of stroke during 1990-2010: findings from the Global Burden of Disease Study 2010. Lancet, 383(9913), 245-254.

Fienberg, S. E., Rinaldo, A., \& Zhou, Y. (2009). On the geometry of discrete exponential families with application to exponential random graph models. Electronic Journal of Statistics, 3, 446-484.

Figini, M., Zucca, I., Aquino, D., Pennacchio, P., Nava, S., Di Marzio, A., Preti, M. G., Baselli, G., Spreafico, R., \& Frassoni, C. (2015). In vivo DTI tractography of the rat brain: An atlas of the main tracts in Paxinos space with histological comparison. Magnetic Resonance Imaging, 33(3), 296-303.

Finan, J. D. (2018). Biomechanical simulation of traumatic brain injury in the rat. Clinical Biomechanics, 64, 114-121.

Fluri, F., Schuhmann, M. K., \& Kleinschnitz, C. (2015). Animal models of ischemic stroke and their application in clinical research. Drug Design, Development and Therapy, 9, 3445-3454. 
Foda, M. A., \& Marmarou, A. (1994). A new model of diffuse brain injury in rats. Part II: Morphological characterization. Journal of Neurosurgery, 80(2), 301-313.

Fornito, A., Zalesky, A., \& Breakspear, M. (2013). Graph analysis of the human connectome: Promise, progress, and pitfalls. Neurolmage, $80,426-444$.

Fox, M. D., Halko, M. A., Eldaief, M. C., \& Pascual-Leone, A. (2012). Measuring and manipulating brain connectivity with resting state functional connectivity magnetic resonance imaging (fcMRI) and transcranial magnetic stimulation (TMS). Neurolmage, 62, 2232-2243.

Fox, M. D., \& Raichle, M. E. (2007). Spontaneous fluctuations in brain activity observed with functional magnetic resonance imaging. Nature Reviews Neuroscience, 8(9), 700-711.

Frank, O., \& Strauss, D. (1986). Markov Graphs. Journal of the American Statistical Association, 81(395), 832-842.

Freeman, L. C. (1977). A set of measures of centrality based on betweenness. Sociometry, 40(1), 3541.

Friston, K. J. (2011). Functional and effective connectivity: A review. Brain Connectivity, 1(1), 13-36.

Gao, Y., Choe, A. S., Stepniewska, I., Li, X., Avison, M. J., \& Anderson, A. W. (2013). Validation of DTI tractography-based measures of primary motor area connectivity in the squirrel monkey brain. PloS One, 8(10), e75065.

Garcia, J. H., Liu, K.-F., \& Ho, K.-L. (1995). Neuronal necrosis after middle cerebral artery occlusion in Wistar rats progresses at different time intervals in the caudoputamen and the cortex. Stroke, 26, 636-643.

Giedd, J. N., Blumenthal, J., Jeffries, N. O., Castellanos, F. X., Liu, H., Zijdenbos, A., Paus, T., Evans, A. C., \& Rapoport, J. L. (1999). Brain development during childhood and adolescence: A longitudinal MRI study. Nature Neuroscience, 2(10), 861-863.

Girard, G., Daducci, A., Petit, L., Thiran, J. P., Whittingstall, K., Deriche, R., Wassermann, D., \& Descoteaux, M. (2017). AxTract: Toward microstructure informed tractography. Human Brain Mapping, 38(11), 5485-5500.

Gong, G., Rosa-Neto, P., Carbonell, F., Chen, Z. J., He, Y., \& Evans, A. C. (2009). Age- and gender-related differences in the cortical anatomical network. The Journal of Neuroscience, 29(50), 15684-15693.

Goñi, J., van den Heuvel, M. P., Avena-Koenigsberger, A., Velez de Mendizabal, N., Betzel, R. F., Griffa, A., Hagmann, P., Corominas-Murtra, B., Thiran, J.-P., \& Sporns, O. (2014). Resting-brain functional connectivity predicted by analytic measures of network communication. Proceedings of the National Academy of Sciences, 111(2), 833-838.

Gozzi, A., \& Schwarz, A. J. (2016). Large-scale functional connectivity networks in the rodent brain. Neurolmage, 127, 496-509.

Granziera, C., Ay, H., Koniak, S. P., Krueger, G., \& Sorensen, A. G. (2012). Diffusion tensor imaging shows structural remodeling of stroke mirror region: Results from a pilot study. European Neurology, 67(6), 370-376. 
Gratton, C., Emi, M. N., Fernando, P., \& D'Esposito, M. (2013). Focal brain lesions to critical locations cause widespread disruption of the modular organization of the brain. Psychologist, 26(3), 194-198.

Grefkes, C., \& Fink, G. R. (2011). Reorganization of cerebral networks after stroke: New insights from neuroimaging with connectivity approaches. Brain, 134(5), 1264-1276.

Grefkes, C., \& Fink, G. R. (2014). Connectivity-based approaches in stroke and recovery of function. The Lancet Neurology, 13(2), 206-216.

Greicius, M. D., Krasnow, B., Reiss, A. L., \& Menon, V. (2003). Functional connectivity in the resting brain: a network analysis of the default mode hypothesis. Proceedings of the National Academy of Sciences, 100(1), 253-258.

Hagmann, P, Sporns, O., Madan, N., Cammoun, L., Pienaar, R., Wedeen, V. J., Meuli, R., Thiran, J.-P., \& Grant, P. E. (2010). White matter maturation reshapes structural connectivity in the late developing human brain. Proceedings of the National Academy of Sciences, 107(44), 19067-19072.

Hagmann, P., Cammoun, L., Gigandet, X., Meuli, R., Honey, C. J., Van Wedeen, J., \& Sporns, O. (2008). Mapping the structural core of human cerebral cortex. PLoS Biology, 6(7), 1479-1493.

Hagmann, P., Kurant, M., Gigandet, X., Thiran, P., Wedeen, V. J., Meuli, R., \& Thiran, J. P. (2007). Mapping human whole-brain structural networks with diffusion MRI. PLOS ONE, 2(7).

Handcock, M. S. (2003). Assessing degeneracy in statistical models of social networks. Journal of the American Statistical Association, 76(39), 33-50.

Hawellek, D. J., Hipp, J. F., Lewis, C. M., Corbetta, M., \& Engel, A. K. (2011). Increased functional connectivity indicates the severity of cognitive impairment in multiple sclerosis. Proceedings of the National Academy of Sciences, 108(47), 19066-19071.

He, B. J., Snyder, A. Z., Zempel, J. M., Smyth, M. D., \& Raichle, M. E. (2008). Electrophysiological correlates of the brain's intrinsic large-scale functional architecture. Proceedings of the National Academy of Sciences, 105(41), 16039-16044.

Heffernan, M. E., Huang, W., Sicard, K. M., Bratane, B. T., Sikoglu, E. M., Zhang, N., Fisher, M., \& King, J. A. (2013). Multi-modal approach for investigating brain and behavior changes in an animal model of traumatic brain injury. Journal of Neurotrauma, 30(11), 1007-1012.

Heimer, L., \& Robards, M. J. (1981). Neuroanatomical Tract-Tracing Methods. Plenum Press.

Herson, P. S., \& Traystman, R. J. (2014). Animal models of stroke: translational potential at present and in 2050. Future Neurology, 9(5), 541-551.

Hidalgo, C. A., Klinger, B., Barabasi, A.-L., \& Hausmann, R. (2007). The product space conditions the development of nations. Science, 317(5837), 482-487.

Hillary, F. G., Slocomb, J., Hills, E. C., Fitzpatrick, N. M., Medaglia, J. D., Wang, J., Good, D. C., \& Wylie, G. R. (2011). Changes in resting connectivity during recovery from severe traumatic brain injury. International Journal of Psychophysiology, 82(1), 115-123.

Hinne, M., Heskes, T., \& van Gerven, M. A. J. (2012). Bayesian inference of whole-brain networks. ArXiv, 1-10. 
Hofman, P. A. M., Stapert, S. Z., van Kroonenburgh, M. J. P. G., Jolles, J., de Kruijk, J., \& Wilmink, J. T. (2001). MR imaging, single-photon emission CT, and neurocognitive performance after mild traumatic brain injury. American Journal of Neuroradiology, 22(3), 441-449.

Honey, C. J., Sporns, O., Cammoun, L., Gigandet, X., Thiran, J. P., Meuli, R., \& Hagmann, P. (2009). Predicting human resting-state functional connectivity from structural connectivity. Proceedings of the National Academy of Sciences, 106(6), 2035-2040.

Hope, T. M. H., Friston, K., Price, C. J., Leff, A. P., Rotshtein, P., \& Bowman, H. (2019). Recovery after stroke: Not so proportional after all? Brain, 142(1), 15-22.

Hoyer, C., Gass, N., Weber-Fahr, W., \& Sartorius, A. (2014). Advantages and challenges of small animal magnetic resonance imaging as a translational tool. Neuropsychobiology, 69(4), 187-201.

Hsu, J. L., Leemans, A., Bai, C. H., Lee, C. H., Tsai, Y. F., Chiu, H. C., \& Chen, W. H. (2008). Gender differences and age-related white matter changes of the human brain: A diffusion tensor imaging study. Neurolmage, 39, 566-577.

Hua, Y., Schallert, T., Keep, R. F., Wu, J., Hoff, J. T., \& Xi, G. (2002). Behavioral tests after intracerebral hemorrhage in the rat. Stroke, 33(10), 2478-2484.

Hugenschmidt, C. E., Peiffer, A. M., Kraft, R. A., Casanova, R., Deibler, A. R., Burdette, J. H., Maldjian, J. A., \& Laurienti, P. J. (2008). Relating imaging indices of white matter integrity and volume in healthy older adults. Cerebral Cortex, 18(2), 433-442.

Hughes, D. G., Jackson, A., Mason, D. L., Berry, E., Hollis, S., \& Yates, D. W. (2004). Abnormalities on magnetic resonance imaging seen acutely following mild traumatic brain injury: Correlation with neuropsychological tests and delayed recovery. Neuroradiology, 46(7), 550-558.

Humphries, M. D., \& Gurney, K. (2008). Network 'Small-World-Ness': A quantitative method for determining canonical network equivalence. PloS One, 3(4), e0002051.

Hunter, D. R., \& Handcock, M. S. (2006). Inference in curved exponential family models for networks. Journal of Computational and Graphical Statistics, 15(3), 565-583.

Hunter, D. R., Handcock, M. S., Butts, C. T., \& Morris, M. (2009). ERGM: A package to fit, simulate and diagnose exponential-family models for networks. Journal of Statistical Software, 24(3), 1-29.

Hutchison, R. M., Womelsdorf, T., Allen, E. A., Bandettini, P. A., Calhoun, V. D., Corbetta, M., Della Penna, S., Duyn, J. H., Glover, G. H., Conzalez-Castillo, J., Handwerker, D. A., Keilholz, S., Kiviniemi, V., Leopold, D. A., de Pasquale, F., Sporns, O., Walter, M., \& Chang, C. (2013). Dynamic functional connectivity: Promise, issues, and interpretations. Neurolmage, 80, 360-378.

Inglese, M., Makani, S., Johnson, G., Cohen, B. A., Silver, J. A., Gonen, O., \& Grossman, R. I. (2005). Diffuse axonal injury in mild traumatic brain injury: a diffusion tensor imaging study. Journal of Neurosurgery, 103(2), 298-303.

Jackson, T. S., \& Read, N. (2010). Theory of minimum spanning trees. I. Mean-field theory and strongly disordered spin-glass model. Physical Review, 81(2), 1-18.

Jaillard, A., Martin, C. D., Garambois, K., François Lebas, J., \& Hommel, M. (2005). Vicarious function within the human primary motor cortex? A longitudinal fMRI stroke study. Brain, 128(5), 1122-1138. 
Janssen, R. J., Hinne, M., Heskes, T., \& van Gerven, M. A. J. (2014). Quantifying uncertainty in brain network measures using Bayesian connectomics. Frontiers in Computational Neuroscience, 8(10), 126.

Jbabdi, S., Lehman, J. F., Haber, S. N., \& Behrens, T. E. (2013). Human and monkey ventral prefrontal fibers use the same organizational principles to reach their targets: tracing versus tractography. Journal of Neuroscience, 33(7), 3190-3201.

Jbabdi, Saad, \& Johansen-Berg, H. (2011). Tractography: where do we go from here? Brain Connectivity, 1(3), 169-183.

Jbabdi, Saad, Sotiropoulos, S. N., Haber, S. N., Essen, D. C. van, \& Behrens, T. E. (2015). Measuring macroscopic brain connections in vivo. Nature Neuroscience, 18(11), 1546-1555.

Jeffers, M. S., Karthikeyan, S., \& Corbett, D. (2018). Does stroke rehabilitation really matter? Part A: Proportional stroke recovery in the rat. Neurorehabilitation and Neural Repair, 32(1), 3-6.

Jenkinson, M., Bannister, P., Brady, M., \& Smith, S. (2002). Improved optimization for the robust and accurate linear registration and motion correction of brain images. Neurolmage, 17, 825-841.

Jenkinson, M., Beckmann, C. F., Behrens, T. E. J., Woolrich, M. W., \& Smith, S. M. (2012). FSL. Neurolmage, 62, 782-790.

Jenkinson, M., \& Smith, S. (2001). A global optimisation method for robust affine registration of brain images. Medical Image Analysis, 5(2), 143-156.

Jensen, J. H., Helpern, J. A., Ramani, A., Lu, H., \& Kaczynski, K. (2005). Diffusional kurtosis imaging: The quantification of non-Gaussian water diffusion by means of magnetic resonance imaging. Magnetic Resonance in Medicine, 53(6), 1432-1440.

Jeurissen, B., Leemans, A., Jones, D. K., Tournier, J.-D., \& Sijbers, J. (2009). Probabilistic fiber tracking using the residual bootstrap with constrained spherical deconvolution MRI. Proc. Intl. Soc. Mag. Reson. Med., 17, 2009.

Jeurissen, B., Descoteaux, M., Mori, S., \& Leemans, A. (2017). Diffusion MRI fiber tractography of the brain. NMR in Biomedicine, e3785, 1-21.

Jeurissen, B., Leemans, A., Tournier, J. D., Jones, D. K., \& Sijbers, J. (2013). Investigating the prevalence of complex fiber configurations in white matter tissue with diffusion magnetic resonance imaging. Human Brain Mapping, 34(11), 2747-2766.

Jeurissen, B., Tournier, J. D., Dhollander, T., Connelly, A., \& Sijbers, J. (2014). Multi-tissue constrained spherical deconvolution for improved analysis of multi-shell diffusion MRI data. Neurolmage, 103, 411-426.

Jiang, L., Xu, H., Yu, C., Jiang, L., Xu, H., \& Yu, C. (2013). Brain connectivity plasticity in the motor network after ischemic stroke. Neural Plasticity, 2013, 1-11.

Jiang, T. (2013). Brainnetome: A new -ome to understand the brain and its disorders. Neurolmage, 80 , 263-272. 
Johansen-Berg, H., Della-Maggiore, V., Behrens, T. E. J., Smith, S. M., \& Paus, T. (2007). Integrity of white matter in the corpus callosum correlates with bimanual co-ordination skills. Neurolmage, 36(Suppl. 2), 16-21.

Johansen-Berg, H., \& Rushworth, M. F. S. (2009). Using diffusion imaging to study human connectional anatomy. Annual Review of Neuroscience, 32, 75-94.

Johansen-Berg, H., Scholz, J., \& Stagg, C. J. (2010). Relevance of structural brain connectivity to learning and recovery from stroke. Frontiers in Systems Neuroscience, 4(11), 146.

Jones, D. (2008). Studying connections in the living human brain with diffusion MRI. Cortex, 44(8), 936-952.

Jones, T. A. (2017). Motor compensation and its effects on neural reorganization after stroke. Nature Reviews Neuroscience, 18, 267-280.

Jones, T. A., Kleim, J. A., \& Greenough, W. T. (1996). Synaptogenesis and dendritic growth in the cortex opposite unilateral sensorimotor cortex damage in adult rats: A quantitative electron microscopic examination. Brain Research, 733(1), 142-148.

Kaiser, M., \& Hilgetag, C. C. (2006). Nonoptimal component placement, but short processing paths, due to long-distance projections in neural systems. PLoS Computational Biology, 2(7), e95.

Kaiser, M., Martin, R., Andras, P., \& Young, M. P. (2007). Simulation of robustness against lesions of cortical networks. European Journal of Neuroscience, 25(10), 3185-3192.

Kasenburg, N., Liptrot, M., Reislev, N. L., Orting, S. N., Nielsen, M., Garde, E., \& Feragen, A. (2016). Training shortest-path tractography: Automatic learning of spatial priors. Neurolmage, 130, 63-76.

Klimm, F., Bassett, D. S., Carlson, J. M., \& Mucha, P. J. (2014). Resolving structural variability in network models and the brain. PLoS Computational Biology, 10(3).

Knösche, T. R., Anwander, A., Liptrot, M., \& Dyrby, T. B. (2015). Validation of tractography: Comparison with manganese tracing. Human Brain Mapping, 36(10), 4116-4134.

Krakauer, J. W., \& Marshall, R. S. (2015). The proportional recovery rule for stroke revisited. Annals of Neurology, 78(6), 845-847.

Kumar, R., Gupta, R. K., Husain, M., Chaudhry, C., Srivastava, A., Saksena, S., \& Rathore, R. K. S. (2009). Comparative evaluation of corpus callosum DTI metrics in acute mild and moderate traumatic brain injury: Its correlation with neuropsychometric test. Brain Injury, 23(7-8), 675-685.

Kundert, R., Goldsmith, J., Veerbeek, J. M., Krakauer, J. W., \& Luft, A. R. (2019). What the proportional recovery rule is (and is not): Methodological and statistical considerations. Neurorehabilitation and Neural Repair, 33(11), 1-12.

La Rosa, P. S., Brooks, T. L., Deych, E., Shands, B., Prior, F., Larson-Prior, L. J., \& Shannon, W. D. (2016). Gibbs distribution for statistical analysis of graphical data with a sample application to fcMRI brain images. Statistics in Medicine, 35(4), 566-580.

Langlois, J. A., Rutland-Brown, W., \& Wald, M. M. (2009). The epidemiology and impact of traumatic brain injury. Journal of Head Trauma Rehabilitation, 11-14. 
LeBihan, D., Breton, E., Lallemand, D., Grenier, P., Cabanis, E., \& Laval-Jeantet, M. (1986). MR imaging of intravoxel incoherent motions: application to diffusion and perfusion in neurologic disorders. Radiology, 161, 401-407.

Lemkaddem, A., Skiöldebrand, D., Dal Palú, A., Thiran, J.-P., \& Daducci, A. (2014). Global tractography with embedded anatomical priors for quantitative connectivity analysis. Frontiers in Neurology, 5(11), 1-13.

Li, Jia, Li, X. Y., Feng, D. F., \& Gu, L. (2011). Quantitative evaluation of microscopic injury with diffusion tensor imaging in a rat model of diffuse axonal injury. European Journal of Neuroscience, 33(5), 933945.

Li, Jing, Zhao, C., Rao, J.-S., Yang, F.-X., Wang, Z.-J., Lei, J.-F., Yang, Z.-Y., \& Li, X.-G. (2017). Structural and metabolic changes in the traumatically injured rat brain: high-resolution in vivo proton magnetic resonance spectroscopy at 7T. Neuroradiology, 59(12), 1203-1212.

Li, L., Chopp, M., Ding, G., Li, Q., Mahmood, A., \& Jiang, Q. (2017). Chronic global analysis of vascular permeability and cerebral blood flow after bone marrow stromal cell treatment of traumatic brain injury in the rat: A long-term MRI study. Brain Research, 1675, 61-70.

Li, S., Sun, Y., Shan, D., Feng, B., Xing, J., Duan, Y., Dai, J., Lei, H., \& Zhou, Y. (2013). Temporal profiles of axonal injury following impact acceleration traumatic brain injury in rats-a comparative study with diffusion tensor imaging and morphological analysis. International Journal of Legal Medicine, 127(1), 159-167.

Li, Y., Chopp, M., Jiang, N., Zhang, Z. G., \& Zaloga, C. (1995). Induction of DNA fragmentation after 10 to 120 minutes of focal cerebral ischemia in rats. Stroke, 26(7), 1252-1258.

Liang, A. C., Mandeville, E. T., Maki, T., Shindo, A., Som, A. T., Egawa, N., Itoh, K., Chuang, T. T., Mcneish, J. D., Holder, J. C., Lok, J., Lo, E. H., \& Arai, K. (2016). Effects of aging on neural stem/progenitor cells and oligodendrocyte precursor cells after focal cerebral ischemia in spontaneously hypertensive rats. Cell Transplantation, 25(4), 705-714.

Lim, D. H., LeDue, J. M., Mohajerani, M. H., \& Murphy, T. H. (2014). Optogenetic mapping after stroke reveals network-wide scaling of functional connections and heterogeneous recovery of the periinfarct. The Journal of Neuroscience, 34(49), 16455-16466.

Lim, S., Han, C. E., Uhlhaas, P. J., \& Kaiser, M. (2015). Preferential detachment during human brain development: Age- and sex-specific structural connectivity in diffusion tensor imaging (DTI) data. Cerebral Cortex, 25(6), 1477-1489.

Lindberg, P. G., Nagy, Z., Skejø, P. H. B., Borg, J., Forssberg, H., Schmitz, C., Rounis, E., Wernegren, H., Bring, A., \& Engardt, M. (2007). Wallerian degeneration of the corticofugal tracts in chronic stroke: A pilot study relating diffusion tensor imaging, transcranial magnetic stimulation, and hand function. Neurorehabilitation and Neural Repair, 21(6), 551-560.

Lindenberg, R., Renga, V., Zhu, L. L., Betzler, F., Alsop, D., \& Schlaug, G. (2010). Structural integrity of corticospinal motor fibers predicts motor impairment in chronic stroke. Neurology, 74(4), 280-287.

Lindenberg, R., Zhu, L. L., \& Schlaug, G. (2013). Predicting functional motor potential in chronic stroke patients using diffusion tensor imaging. Human Brain Mapping, 33(5), 617-632. 
Ling, J. M., Peña, A., Yeo, R. A., Merideth, F. L., Klimaj, S., Gasparovic, C., \& Mayer, A. R. (2012). Biomarkers of increased diffusion anisotropy in semi-acute mild traumatic brain injury: A longitudinal perspective. Brain, 135(4), 1281-1292.

Liu, F., Yuan, R., Benashski, S. E., \& McCullough, L. D. (2009). Changes in experimental stroke outcome across the lifespan. Journal of Cerebral Blood Flow \& Metabolism, 29(4), 792-802.

Liu, Z., Li, Y., Zhang, X., Savant-Bhonsale, S., \& Chopp, M. (2008). Contralesional axonal remodeling of the corticospinal system in adult rats after stroke and bone marrow stromal cell treatment. Stroke, 39(9), 2571-2577.

Long, J. A., Watts, L. T., Chemello, J., Huang, S., Shen, Q., \& Duong, T. Q. (2015). Multiparametric and longitudinal MRI characterization of mild traumatic brain injury in rats. Journal of Neurotrauma, 32(8), 598-607.

Longa, E. Z., Weinstein, P. R., Carlson, S., \& Cummins, R. (1989). Reversible middle cerebral artery occlusion without craniectomy in rats. Stroke, 20(1), 84-91.

Louviere, J. J., Hensher, D. A., \& Swait, J. D. (2000). Stated Choice Methods. New York: Cambridge University Press.

Lu, H., Zou, Q., Gu, H., Raichle, M. E., Stein, E. A., \& Yang, Y. (2012). Rat brains also have a default mode network. Proceedings of the National Academy of Sciences, 109(10), 3979-3984.

Maas, A. I., Stocchetti, N., \& Bullock, R. (2008). Moderate and severe traumatic brain injury in adults. The Lancet Neurology, 7(8), 728-741.

Magri, C., Schridde, U., Murayama, Y., Panzeri, S., \& Logothetis, N. K. (2012). The amplitude and timing of the BOLD signal reflects the relationship between local field potential power at different frequencies. Journal of Neuroscience, 32(4), 1395-1407.

Maier-Hein, K. H. (2017). The challenge of mapping the human connectome based on diffusion tractography. Nature Communications, 8(1).

Maier-Hein, K. H., Neher, P., Houde, J.-C., \& Côté, M.-A. (2016). Tractography-based connectomes are dominated by false-positive connections. BioRxiv, 1-23.

Majka, P., Kublik, E., Furga, G., \& Wójcik, D. K. (2012). Common atlas format and 3D brain atlas reconstructor: Infrastructure for Constructing 3D brain atlases. Neuroinformatics, 10(2), 181-197.

Mangin, J. F., Fillard, P., Cointepas, Y., Le Bihan, D., Frouin, V., \& Poupon, C. (2013). Toward global tractography. Neurolmage, 80, 290-296.

Marcel, B.-R., Carmel, J. B., Chakrabarty, S., \& Martin, J. H. (2007). Electrical stimulation of spared corticospinal axons augments connections with ipsilateral spinal motor circuits after injury. Journal of Neuroscience, 27(50), 13793-13801.

Marmarou, A., Foda, M. A., van den Brink, W., Campbell, J., Kita, H., \& Demetriadou, K. (1994). A new model of diffuse brain injury in rats. Part I: Pathophysiology and biomechanics. Journal of Neurosurgery, 80(2), 291-300. 
Masel, B. E., \& DeWitt, D. S. (2010). Traumatic brain injury: A disease process, not an event. Journal of Neurotrauma, 27(8), 1529-1540.

Maslov, S., \& Sneppen, K. (2002). Specificity and stability in topology of protein networks. Science, 296, 910-914.

Mayer, A. R., Ling, J., Mannell, M. V, Gasparovic, C., Phillips, J. P., Doezema, D., Reichard, R., \& Yeo, R. A. (2010). A prospective diffusion tensor imaging study in mild traumatic brain injury. Neurology, 74(8), 643-650.

Mayer, A. R., Mannell, M. V., Ling, J., Gasparovic, C., \& Yeo, R. A. (2011). Functional connectivity in mild traumatic brain injury. Human Brain Mapping, 32(11), 1825-1835.

McMahon, P. J., Hricik, A., Yue, J. K., Puccio, A. M., Inoue, T., Lingsma, H. F., Beers, S. R., Gordon, W. A., Valadka, A. B., Manley, G. T., Okonkwo, D. O., Casey, S. S., Cooper, S. R., Dams-O'Connor, K., Menon, D. K., Sorani, M. D., Yuh, E. L., Mukherjee, P., Schnyer, D. M., \& Vassar, M. J. (2013). Symptomatology and functional outcome in mild traumatic brain injury: Results from the Prospective TRACK-TBI Study. Journal of Neurotrauma, 31(1), 26-33.

Meghanathan, N. (2015). Intelligent Systems in Cybernetics and Automation Theory. 348, 11-20.

Meier-Ruge, W., Ulrich, J., Brühlmann, M., \& Meier, E. (1992). Age-related white matter atrophy in the human brain. Annals of the New York Academy of Sciences, 673, 260-269.

Meier, T. B., Desphande, A. S., Vergun, S., Nair, V. A., Song, J., Biswal, B. B., Meyerand, M. E., Birn, R. M., \& Prabhakaran, V. (2012). Support vector machine classification and characterization of agerelated reorganization of functional brain networks. Neurolmage, 60, 601-613.

Mengler, L., Khmelinskii, A., Diedenhofen, M., Po, C., Staring, M., Lelieveldt, B. P. F., \& Hoehn, M. (2014). Brain maturation of the adolescent rat cortex and striatum: Changes in volume and myelination. Neurolmage, 84, 35-44.

Metz, G. A., \& Whishaw, I. Q. (2002). Cortical and subcortical lesions impair skilled walking in the ladder rung walking test: a new task to evaluate fore- and hindlimb stepping, placing, and coordination. Journal of Neuroscience Methods, 115, 169-179.

Meunier, D., Achard, S., Morcom, A., \& Bullmore, E. (2009). Age-related changes in modular organization of human brain functional networks. Neurolmage, 44, 715-723.

Montembeault, M., Joubert, S., Doyon, J., Carrier, J., Gagnon, J. F., Monchi, O., Lungu, O., Belleville, S., \& Brambati, S. M. (2012). The impact of aging on gray matter structural covariance networks. Neurolmage, 63, 754-759.

Mori, S, Crain, B. J., Chacko, V. P., \& van Zijl, P. C. (1999). Three-dimensional tracking of axonal projections in the brain by magnetic resonance imaging. Annals of Neurology, 45(2), 265-269.

Mori, Susumu, \& Zhang, J. (2006). Principles of diffusion tensor imaging and its applications to basic neuroscience research. Neuron, 51(5), 527-539.

Morris, M., Handcock, M. S., \& Hunter, D. R. (2008). Specification of exponential-family random graph models. Journal of Statistical Software, 24(4). 
Murphy, K., Birn, R. M., Handwerker, D. A., Jones, T. B., \& Bandettini, P. A. (2009). The impact of global signal regression on resting state correlations: Are anti-correlated networks introduced? Neurolmage, 44, 893-905.

Murphy, K., \& Fox, M. D. (2017). Towards a consensus regarding global signal regression for resting state functional connectivity MRI. Neurolmage, 154, 169-173.

Murphy, T. H., \& Corbett, D. (2009). Plasticity during stroke recovery: from synapse to behaviour. Nature Reviews Neuroscience, 10(12), 861-872.

Murray, I., Ghahramani, Z., \& MacKay, D. J. C. (2006). MCMC for doubly-intractable distributions. Citeseer.

Neher, P. F., Côté, M. A., Houde, J. C., Descoteaux, M., \& Maier-Hein, K. H. (2017). Fiber tractography using machine learning. Neurolmage, 158, 417-429.

Newman, M. E. J. (2003). The structure and function of complex networks. Society for Industrial and Applied Mathematics Review, 45(2), 167-256.

Niccoli, T., \& Partridge, L. (2012). Ageing as a risk factor for disease. Current Biology, 22(17), R741-52.

NIH. (2014). BRAIN 2025: A scientific vision Final report of the ACD BRAIN working group.

Oh, S. W., Harris, J. A., Ng, L., Winslow, B., Cain, N., Mihalas, S., Wang, Q., Lau, C., Kuan, L., Henry, A. M., Mortrud, M. T., Ouellette, B., Nguyen, T. N., Sorensen, S. A., Slaughterbeck, C. R., Wakeman, W., Li, Y., Feng, D., Ho, A., ... Zeng, H. (2014). A mesoscale connectome of the mouse brain. Nature, 508(7495), 207-214.

Okabe, N., Shiromoto, T., Himi, N., Lu, F., Maruyama-Nakamura, E., Narita, K., Iwachidou, N., Yagita, Y., \& Miyamoto, O. (2016). Neural network remodeling underlying motor map reorganization induced by rehabilitative training after ischemic stroke. Neuroscience, 339, 338-362.

Ortega, G. J., Sola, R. G., \& Pastor, J. (2008). Complex network analysis of human ECoG data. Neuroscience Letters, 447(2-3), 129-133.

Otte, W. M., van Diessen, E., Paul, S., Ramaswamy, R., Subramanyam Rallabandi, V. P., Stam, C. J., \& Roy, P. K. (2015). Aging alterations in whole-brain networks during adulthood mapped with the minimum spanning tree indices: The interplay of density, connectivity cost and life-time trajectory. Neurolmage, 109, 171-189.

Paasonen, J., Stenroos, P., Salo, R. A., Kiviniemi, V., \& Gröhn, O. (2018). Functional connectivity under six anesthesia protocols and the awake condition in rat brain. Neurolmage, 172, 9-20.

Palacios, E. M., Sala-Llonch, R., Junque, C., Roig, T., Tormos, J. M., Bargallo, N., \& Vendrell, P. (2012). White matter integrity related to functional working memory networks in traumatic brain injury. Neurology, 78(12), 852-860.

Palacios, Eva M., Sala-Llonch, R., Junque, C., Roig, T., Tormos, J. M., Bargallo, N., \& Vendrell, P. (2013). Resting-state functional magnetic resonance imaging activity and connectivity and cognitive outcome in traumatic brain injury. JAMA Neurology, 70(7), 845-851. 
Pan, W.-J., Thompson, G., Magnuson, M., Majeed, W., Jaeger, D., \& Keilholz, S. (2011). Broadband local field potentials correlate with spontaneous fluctuations in functional magnetic resonance imaging signals in the rat somatosensory cortex under isoflurane anesthesia. Brain Connectivity, 1(2), 119-131.

Pattison, P., \& Wasserman, S. (1996). Logit models and logistic regressions for social networks: I. An introduction to Markov Graphs and P*. Psychometrika, 61(3), 401-425.

Paxinos, G., \& Watson, W. (2005). The rat brain in stereotaxic coordinates 5th edition. Elsevier Academic Press, Amsterdam.

Poldrack, R. A., \& Farah, M. J. (2015). Progress and challenges in probing the human brain. Nature, 526(7573), 371-379.

Preti, M. G., Bolton, T. A., \& van de Ville, D. (2017). The dynamic functional connectome: State-of-theart and perspectives. Neurolmage, 160, 41-54.

Raffelt, D. A., Smith, R. E., Ridgway, G. R., Tournier, J. D., Vaughan, D. N., Rose, S., Henderson, R., \& Connelly, A. (2015). Connectivity-based fixel enhancement: Whole-brain statistical analysis of diffusion MRI measures in the presence of crossing fibres. Neurolmage, 117, 40-55.

Raffelt, D. A., Tournier, J. D., Smith, R. E., Vaughan, D. N., Jackson, G., Ridgway, G. R., \& Connelly, A. (2017). Investigating white matter fibre density and morphology using fixel-based analysis. Neurolmage, 144, 58-73.

Raffelt, D., Tournier, J. D., Rose, S., Ridgway, G. R., Henderson, R., Crozier, S., Salvado, O., \& Connelly, A. (2012). Apparent Fibre Density: A novel measure for the analysis of diffusion-weighted magnetic resonance images. Neurolmage, 59, 3976-3994.

Raftery, A. E. (1995). Bayesian model selection in social research. Sociological Methodology, 25, 111163.

Reglodi, D., Tamas, A., \& Lengvari, I. (2003). Examination of sensorimotor performance following middle cerebral artery occlusion in rats. Brain Research Bulletin, 59(6), 459-466.

Rehme, A. K., Eickhoff, S. B., Rottschy, C., Fink, G. R., \& Grefkes, C. (2012). Activation likelihood estimation meta-analysis of motor-related neural activity after stroke. Neurolmage, 59, 2771-2782.

Rehme, A. K., \& Grefkes, C. (2013). Cerebral network disorders after stroke: Evidence from imagingbased connectivity analyses of active and resting brain states in humans. Journal of Physiology, 591(1), $17-31$.

Reisert, M., Mader, I., Anastasopoulos, C., Weigel, M., Schnell, S., \& Kiselev, V. (2011). Global fiber reconstruction becomes practical. Neurolmage, 54, 955-962.

Reveley, C., Seth, A. K., Pierpaoli, C., Silva, A. C., Yu, D., Saunders, R. C., Leopold, D. A., \& Ye, F. Q. (2015). Superficial white matter fiber systems impede detection of long-range cortical connections in diffusion MR tractography. Proceedings of the National Academy of Sciences, 112(21), E2820-E2828.

Robins, G., Pattison, P., Kalish, Y., \& Lusher, D. (2007). An introduction to exponential random graph $\left(p^{*}\right)$ models for social networks. Social Networks, 29(2), 173-191. 
Robins, G., Snijders, T., Wang, P., Handcock, M., \& Pattison, P. (2007). Recent developments in exponential random graph ( $\left.p^{*}\right)$ models for social networks. Social Networks, 29(2), 192-215.

Roelofs, T. J. M., Verharen, J. P. H., van Tilborg, G. A. F., Boekhoudt, L., van der Toorn, A., de Jong, J. W., Luijendijk, M. C. M., Otte, W. M., Adan, R. A. H., \& Dijkhuizen, R. M. (2017). A novel approach to map induced activation of neuronal networks using chemogenetics and functional neuroimaging in rats: A proof-of-concept study on the mesocorticolimbic system. Neurolmage, 156, 109-118.

Roine, T., Jeurissen, B., Perrone, D., Aelterman, J., Philips, W., Sijbers, J., \& Leemans, A. (2019). Reproducibility and intercorrelation of graph theoretical measures in structural brain connectivity networks. Medical Image Analysis, 52, 56-67.

Roozenbeek, B., Maas, A. I. R., \& Menon, D. K. (2013). Changing patterns in the epidemiology of traumatic brain injury. Nature Reviews Neurology, 9(4), 231-236.

Rosas, H. D., Lee, S. Y., Bender, A. C., Zaleta, A. K., Vangel, M., Yu, P., Fischl, B., Pappu, V., Onorato, C., Cha, J. H., Salat, D. H., \& Hersch, S. M. (2010). Altered white matter microstructure in the corpus callosum in Huntington's disease: Implications for cortical "disconnection". Neurolmage, 49, 29953004.

Rouder, J. N., Speckman, P. L., Sun, D., Morey, R. D., \& Iverson, G. (2009). Bayesian t-tests for accepting and rejecting the null hypothesis. Psychonomic Bulletin \& Review, 16(2), 225-237.

Ruber, T., Lindenberg, R., \& Schlaug, G. (2012). Compensatory role of the cortico-rubro-spinal tract in motor recovery after stroke. Neurology, 78, P06.243-P06.243.

Rubinov, M., \& Sporns, O. (2010). Complex network measures of brain connectivity: Uses and interpretations. Neurolmage, 52, 1059-1069.

Rutgers, D. R., Toulgoat, F., Cazejust, J., Fillard, P., Lasjaunias, P., \& Ducreux, D. (2008). White matter abnormalities in mild traumatic brain injury: A diffusion tensor imaging study. American Journal of Neuroradiology, 29(3), 514-519.

Sala-Llonch, R., Junqué, C., Arenaza-Urquijo, E. M., Vidal-Piñeiro, D., Valls-Pedret, C., Palacios, E. M., Domènech, S., Salvà, A., Bargalló, N., \& Bartrés-Faz, D. (2014). Changes in whole-brain functional networks and memory performance in aging. Neurobiology of Aging, 35(10), 2193-2202.

Salat, D. H., Tuch, D. S., Greve, D. N., van der Kouwe, A. J. W., Hevelone, N. D., Zaleta, A. K., Rosen, B. R., Fischl, B., Corkin, S., Diana Rosas, H., \& Dale, A. M. (2005). Age-related alterations in white matter microstructure measured by diffusion tensor imaging. Neurobiology of Aging, 26(8), 1215-1227.

Salat, David H. (2011). The declining infrastructure of the aging brain. Brain Connectivity, 1(4), 279293.

Sarwar, T., Ramamohanarao, K., \& Zalesky, A. (2019). Mapping connectomes with diffusion MRI: deterministic or probabilistic tractography? Magnetic Resonance in Medicine, 81(2), 1368-1384.

Schaechter, J. D., Fricker, Z. P., Perdue, K. L., Helmer, G., Vangel, M. G., Greve, D. N., \& Makris, N. (2010). Microstructural status of ipsilesional and contralesional corticospinal tract correlates with motor skill in chronic stroke patients. Human Brain Mapping, 30(11), 3461-3474. 
Schiavi, S., Barakovic, M., Ocampo-Pineda, M., Descoteaux, M., Thiran, J., \& Daducci, A. (2019). Reducing false positives in tractography with microstructural and anatomical priors. BioRxiv, 1-13.

Schilling, K. G., Gao, Y., Stepniewska, I., Janve, V., Landman, B. A., \& Anderson, A. W. (2019). Anatomical accuracy of standard-practice tractography algorithms in the motor system - A histological validation in the squirrel monkey brain. Magnetic Resonance Imaging, 55(9), 7-25.

Schilling, K. G., Janve, V., Gao, Y., Stepniewska, I., Landman, B. A., \& Anderson, A. W. (2018). Histological validation of diffusion MRI fiber orientation distributions and dispersion. Neurolmage, $165,200-221$.

Schmitt, O., \& Eipert, P. (2012). neuroVIISAS: Approaching multiscale simulation of the rat connectome. Neuroinformatics, 10(3), 243-267.

Sharp, D. J., Beckmann, C. F., Greenwood, R., Kinnunen, K. M., Bonnelle, V., De Boissezon, X., Powell, J. H., Counsell, S. J., Patel, M. C., \& Leech, R. (2011). Default mode network functional and structural connectivity after traumatic brain injury. Brain, 134(8), 2233-2247.

Sharp, D. J., Scott, G., \& Leech, R. (2014). Network dysfunction after traumatic brain injury. Nature Reviews Neurology, 10(3), 156-166.

Shen, K., Bezgin, G., Hutchison, R. M., Gati, J. S., Menon, R. S., Everling, S., \& McIntosh, A. R. (2012). Information processing architecture of functionally defined clusters in the macaque cortex. Journal of Neuroscience, 32(48), 17465-17476.

Shmuel, A., \& Leopold, D. A. (2008). Neuronal Correlates of Spontaneous Fluctuations in fMRI Signals in Monkey Visual Cortex : Implications for Functional Connectivity at Rest. Human Brain Mapping, 29, 751-761.

Sierakowiak, A., Monnot, C., Aski, S. N., Uppman, M., Li, T. Q., Damberg, P., \& Brené, S. (2015). Default mode network, motor network, dorsal and ventral basal ganglia networks in the rat brain: Comparison to human networks using resting state-fMRI. PLOS ONE, 10(3), 1-20.

Simpson S. L., \& Laurienti, P. J. (2015). Disentangling brain graphs: A note on the conflation of network and connectivity analyses. Brain Connectivity, 6(2), 95-98.

Simpson, S. L., \& Laurienti, P. J. (2015). A two-part mixed-effects modeling framework for analyzing whole-brain network data. Neurolmage, 113, 310-319.

Simpson, S. L., Hayasaka, S., \& Laurienti, P. J. (2011). Exponential random graph modeling for complex brain networks. PLOS ONE, 6(5).

Simpson, S. L., Moussa, M. N., \& Laurienti, P. J. (2012). An exponential random graph modeling approach to creating group-based representative whole-brain connectivity networks. Neurolmage, $60,1117-1126$.

Simpson, S. L., DuBois Bowman, F., \& Laurienti, P. J. (2014). NIH Public Access. Statistics Surveys, 7, 136.

Singh, K., Trivedi, R., Devi, M. M., Tripathi, R. P., \& Khushu, S. (2016). Longitudinal changes in the DTI measures, anti-GFAP expression and levels of serum inflammatory cytokines following mild traumatic brain injury. Experimental Neurology, 275, 427-435. 
Sinke, M. R. T., Buitenhuis, J. W., van der Maas, F., Nwiboko, J., Dijkhuizen, R. M., van Diessen, E., \& Otte, W. M. (2019). The power of language: Functional brain network topology of deaf and hearing in relation to sign language experience. Hearing Research, 373, 32-47.

Sinke, M. R. T., Dijkhuizen, R. M., Caimo, A., Stam, C. J., \& Otte, W. M. (2016). Bayesian exponential random graph modeling of whole-brain structural networks across lifespan. Neurolmage, 135, 79-91.

Sinke, M. R. T., Otte, W. M., Christiaens, D., Schmitt, O., Leemans, A., van der Toorn, A., Sarabdjitsingh, R. A., Joëls, M., \& Dijkhuizen, R. M. (2018). Diffusion MRI-based cortical connectome reconstruction: dependency on tractography procedures and neuroanatomical characteristics. Brain Structure and Function, 223(5), 2269-2285.

Smit, D., De Geus, E., Boersma, M., Boomsma, D., \& Stam, C. (2016). Life-span development of brain network integration assessed with Phase Lag Index connectivity and minimum spanning tree graphs. Brain Connectivity, 6(4), 312-325.

Smith, R. E., Tournier, J. D., Calamante, F., \& Connelly, A. (2012). Anatomically-constrained tractography: Improved diffusion MRI streamlines tractography through effective use of anatomical information. Neurolmage, 62, 1924-1938.

Smith, R. E., Tournier, J. D., Calamante, F., \& Connelly, A. (2013). SIFT: Spherical-deconvolution informed filtering of tractograms. Neurolmage, 67, 298-312.

Smith, R. E., Tournier, J. D., Calamante, F., \& Connelly, A. (2015a). SIFT2: Enabling dense quantitative assessment of brain white matter connectivity using streamlines tractography. Neurolmage, 119, 338-351.

Smith, R. E., Tournier, J. D., Calamante, F., \& Connelly, A. (2015b). The effects of SIFT on the reproducibility and biological accuracy of the structural connectome. Neurolmage, 104, 253-265.

Smith, S. M. (2002). Fast robust automated brain extraction. Human Brain Mapping, 17(3), 143-155.

Snijders, T. A. B., Pattison, P. E., Robins, G. L., \& Handcock, M. S. (2006). New specifications for exponential random graph models. Sociological Methodology, 36, 99-153.

Song, S.-K., Le, T. Q., Lin, S.-J., Sun, S.-W., Cross, A. H., Yoshino, J., \& Armstrong, R. C. (2005). Demyelination increases radial diffusivity in corpus callosum of mouse brain. Neurolmage, 26, 132140.

Sousa, R. M., Rodriguez, J. J. L., Ferri, C. P., Sosa, A. L., Guerra, M., Prince, M., Acosta, D., Jotheeswaran, A., Salas, A., Jacob, K., Pichardo, G. R., Rodriguez, M. C., Huang, Y., \& Albanese, E. (2009). Contribution of chronic diseases to disability in elderly people in countries with low and middle incomes: a 10/66 Dementia Research Group population-based survey. The Lancet, 374(9704), 1821-1830.

Sporns, O. (2010). Networks of the Brain. MIT Press.

Sporns, O., Honey, C. J., \& Kötter, R. (2007). Identification and classification of hubs in brain networks. PLOS ONE, 2(10), e1049.

Sporns, O., \& Kötter, R. (2004). Motifs in brain networks. PLoS Biology, 2(11), e369. 
Sporns, O., Tononi, G., \& Kötter, R. (2005). The human connectome: A structural description of the human brain. PLoS Computational Biology, 1(4), e42.

Spreng, R. N., \& Schacter, D. L. (2012). Default network modulation and large-scale network interactivity in healthy young and old adults. Cerebral Cortex, 22(11), 2610-2621.

Stam, C. J., \& van Straaten, E. C. W. (2012). The organization of physiological brain networks. Clinical Neurophysiology, 123(6), 1067-1087.

Stam, C. J., Haan, W. de, Daffertshofer, A., Jones, B. F., Manshanden, I., Walsum, A. M. V. C. van, Montez, T., Verbunt, J. P. A., Munck, J. C. de, Dijk, B. W. van, Berendse, H. W., \& Scheltens, P. (2009). Graph theoretical analysis of magnetoencephalographic functional connectivity in Alzheimer 's disease. Brain, 132, 213-224.

Stam, C. J., Tewarie, P., van Dellen, E., van Straaten, E. C. W., Hillebrand, A., \& van Mieghem, P. (2014). The trees and the forest: Characterization of complex brain networks with minimum spanning trees. International Journal of Psychophysiology, 92(3), 129-138.

Stam, C. J. (2014). Modern network science of neurological disorders. Nature Reviews Neuroscience, 15(10), 683-695.

Stephan, K. E., Kamper, L., Bozkurt, A., Burns, G. A., Young, M. P., \& Kötter, R. (2001). Advanced database methodology for the Collation of Connectivity data on the Macaque brain (CoCoMac). Philosophical Transactions of the Royal Society of London Series B, Biological Sciences, 356(1412), 1159-1186.

Stillman, P. E., Wilson, J. D., Denny, M. J., Desmarais, B. A., Bhamidi, S., Cranmer, S. J., \& Lu, Z. L. (2017). Statistical modeling of the default mode brain network reveals a segregated highway structure. Scientific Reports, 7(1), 1-14.

Stinear, C. M., Barber, P. A., Smale, P. R., Coxon, J. P., Fleming, M. K., \& Byblow, W. D. (2007). Functional potential in chronic stroke patients depends on corticospinal tract integrity. Brain, 130(1), 170-180.

Straathof, M., Sinke, M. R. T., Dijkhuizen, R. M., \& Otte, W. M. (2019). A systematic review on the quantitative relationship between structural and functional network connectivity strength in mammalian brains. Journal of Cerebral Blood Flow and Metabolism, 39(2), 189-209.

Straathof, M., Sinke, M. R. T., Roelofs, T. J. M., Blezer, E. L. A., Sarabdjitsingh, R. A., van der Toorn, A., Schmitt, O., Otte, W. M., \& Dijkhuizen, R. M. (2020). Distinct structure-function relationships across cortical regions and connectivity scales in the rat brain. Scientific Reports, 10(1), 1-14.

Strauss, D., \& Ikeda, M. (1990). Pseudolikelihood estimation for social networks. Journal of the American Statistical Association, 85(409), 204-212.

Stroemer, R. P., Kent, T. A., \& Hulsebosch, C. E. (1995). Neocortical neural sprouting, synaptogensis, and behavioral recovery after neocortical infarction in rats. Stroke, 26, 2135-2144.

Sun, S. W., Liang, H. F., Trinkaus, K., Cross, A. H., Armstrong, R. C., \& Song, S. K. (2006). Noninvasive detection of cuprizone induced axonal damage and demyelination in the mouse corpus callosum. Magnetic Resonance in Medicine, 55(2), 302-308. 
Tagliaferri, F., Compagnone, C., Korsic, M., Servadei, F., \& Kraus, J. (2006). A systematic review of brain injury epidemiology in Europe. Acta Neurochirurgica, 148(3), 255-267.

Takenobu, Y., Hayashi, T., Moriwaki, H., Nagatsuka, K., Naritomi, H., \& Fukuyama, H. (2014). Motor recovery and microstructural change in rubro-spinal tract in subcortical stroke. Neurolmage: Clinical, 4, 201-208.

Taki, Y., Kinomura, S., Sato, K., Goto, R., Kawashima, R., \& Fukuda, H. (2011). A longitudinal study of gray matter volume decline with age and modifying factors. Neurobiology of Aging, 32(5), 907-915.

Tax, C. M. W., Jeurissen, B., Vos, S. B., Viergever, M. A., \& Leemans, A. (2014). Recursive calibration of the fiber response function for spherical deconvolution of diffusion MRI data. Neurolmage, 86, 6780 .

Telesford, Q. K., Simpson, S. L., Burdette, J. H., Hayasaka, S., \& Laurienti, P. J. (2011). The brain as a complex system: Using network science as a tool for understanding the brain. Brain Connectivity, 1(4), 295-308.

Tewarie, P., van Dellen, E., Hillebrand, A., \& Stam, C. J. (2015). The minimum spanning tree: An unbiased method for brain network analysis. Neurolmage, 104, 177-188.

Tewarie, P., Schoonheim, M. M., Schouten, D. I., Polman, C. H., Balk, L. J., Uitdehaag, B. M. J., Geurts, J. J. G., Hillebrand, A., Barkhof, F., \& Stam, C. J. (2015). Functional brain networks: Linking thalamic atrophy to clinical disability in multiple sclerosis, a multimodal fMRI and MEG Study. Human Brain Mapping, 36(2), 603-618.

Thomas, C., Ye, F. Q., Irfanoglu, M. O., Modi, P., Saleem, K. S., Leopold, D. A., \& Pierpaoli, C. (2014). Anatomical accuracy of brain connections derived from diffusion MRI tractography is inherently limited. Proceedings of the National Academy of Sciences, 111(46), 16574-16579.

Tournier, J. D., Mori, S., \& Leemans, A. (2011). Diffusion tensor imaging and beyond. Magnetic Resonance in Medicine, 65(6), 1532-1556.

Tournier, J. D., Calamante, F., \& Connelly, A. (2007). Robust determination of the fibre orientation distribution in diffusion MRI: Non-negativity constrained super-resolved spherical deconvolution. Neurolmage, 35, 1459-1472.

Tournier, J. D., Calamante, F., \& Connelly, A. (2010). Improved probabilistic streamlines tractography by 2 nd order integration over fibre orientation distributions. Proc. ISMRM, 88(2003), 2010.

Tournier, J. D., Calamante, F., \& Connelly, A. (2012). MRtrix: Diffusion tractography in crossing fiber regions. International Journal of Imaging Systems and Technology, 22(1), 53-66.

Tournier, J. D., Calamante, F., Gadian, D. G., \& Connelly, A. (2004). Direct estimation of the fiber orientation density function from diffusion-weighted MRI data using spherical deconvolution. Neurolmage, 23, 1176-1185.

Tu, T.-W., Williams, R. A., Lescher, J. D., Jikaria, N., Turtzo, L. C., \& Frank, J. A. (2016). Radiologicalpathological correlation of diffusion tensor and magnetization transfer imaging in a closed head traumatic brain injury model. Annals of Neurology, 79(6), 907-920.

Tu, W., \& Zhang, N. (2019). How brain reacts to attack at a local region. bioRxiv. 
Tuor, U. I., Morgunov, M., Sule, M., Qiao, M., Clark, D., Rushforth, D., Foniok, T., \& Kirton, A. (2014). Cellular correlates of longitudinal diffusion tensor imaging of axonal degeneration following hypoxicischemic cerebral infarction in neonatal rats. Neurolmage: Clinical, 6, 32-42.

van de Looij, Y., Mauconduit, F., Beaumont, M., Valable, S., Farion, R., Francony, G., Payen, J. F., \& Lahrech, H. (2012). Diffusion tensor imaging of diffuse axonal injury in a rat brain trauma model. NMR in Biomedicine, 25(1), 93-103.

van Dellen, E., Douw, L., Hillebrand, A., de Witt Hamer, P. C., Baayen, J. C., Heimans, J. J., Reijneveld, J. C., \& Stam, C. J. (2014). Epilepsy surgery outcome and functional network alterations in longitudinal MEG: A minimum spanning tree analysis. Neurolmage, 86, 354-363.

van den Heuvel, M. P., Bullmore, E. T., \& Sporns, O. (2016). Comparative Connectomics. Trends in Cognitive Sciences, 20(5), 345-361.

van den Heuvel, M. P., de Reus, M. A., Feldman Barrett, L., Scholtens, L. H., Coopmans, F. M. T., Schmidt, R., Preuss, T. M., Rilling, J. K., \& Li, L. (2015). Comparison of diffusion tractography and tracttracing measures of connectivity strength in rhesus macaque connectome. Human Brain Mapping, 36(8), 3064-3075.

van den Heuvel, M. P., \& Hulshoff Pol, H. E. (2010). Exploring the brain network: A review on restingstate fMRI functional connectivity. European Neuropsychopharmacology, 20(8), 519-534.

van den Heuvel, M. P., Kahn, R. S., Goñi, J., \& Sporns, O. (2012). Brain communication. Proceedings of the National Academy of Sciences, 109, 11372-11377.

van den Heuvel, M. P., Mandl, C. W., Stam, C. J., Kahn, S., \& Pol, H. E. H. (2010). Aberrant frontal and temporal complex network structure in schizophrenia : A graph theoretical analysis. The Journal of Neuroscience, 30(47), 15915-15926.

van den Heuvel, M. P., Scholtens, L. H., \& de Reus, M. A. (2016). Topological organization of connectivity strength in the rat connectome. Brain Structure and Function, 221(3), 1719-1736.

van der Zijden, J. P., Bouts, M. J., Wu, O., Roeling, T. A., Bleys, R. L., van der Toorn, A., \& Dijkhuizen, R. M. (2008). Manganese-enhanced MRI of brain plasticity in relation to functional recovery after experimental stroke. Journal of Cerebral Blood Flow and Metabolism, 28(4), 832-840.

van Diessen, E., Numan, T., van Dellen, E., van der Kooi, A. W., Boersma, M., Hofman, D., van Lutterveld, R., van Dijk, B. W., van Straaten, E. C. W., Hillebrand, A., \& Stam, C. J. (2015). Opportunities and methodological challenges in EEG and MEG resting state functional brain network research. Clinical Neurophysiology, 126(8), 1468-1481.

van Diessen, Eric, Otte, W. M., Stam, C. J., Braun, K. P. J., \& Jansen, F. E. (2016). Electroencephalography based functional networks in newly diagnosed childhood epilepsies. Clinical Neurophysiology, 127(6), 2325-2332.

van Essen, D. C., Ugurbil, K., Auerbach, E., Barch, D., Behrens, T. E. J., Bucholz, R., Chang, A., Chen, L., Corbetta, M., Curtiss, S. W., Della Penna, S., Feinberg, D., Glasser, M. F., Harel, N., Heath, A. C., LarsonPrior, L., Marcus, D., Michalareas, G., Moeller, S., ... Yacoub, E. (2012). The human connectome project: A data acquisition perspective. Neurolmage, 62, 2222-2231. 
van Haren, N. E. M., Pol, H. E. H., Schnack, H. G., Cahn, W., Brans, R., Carati, I., Rais, M., \& Kahn, R. S. (2008). Progressive brain volume loss in schizophrenia over the course of the illness: Evidence of maturational abnormalities in early adulthood. Biological Psychiatry, 63(1), 106-113.

van Meer, M. P. A., van der Marel, K., Wang, K., Otte, W. M., el Bouazati, S., Roeling, T. A. P., Viergever, M. A., Berkelbach van der Sprenkel, J. W., \& Dijkhuizen, R. M. (2010). Recovery of sensorimotor function after experimental stroke correlates with restoration of resting-state interhemispheric functional connectivity. Journal of Neuroscience, 30(11), 3964-3972.

van Meer, M. P. A., Otte, W. M., van der Marel, K., Nijboer, C. H., Kavelaars, A., Berkelbach van der Sprenkel, J. W., Viergever, M. A., \& Dijkhuizen, R. M. (2012). Extent of bilateral neuronal network reorganization and functional recovery in relation to stroke severity. Journal of Neuroscience, 32(13), 4495-4507.

van Meer, M. P. A., van der Marel, K., Otte, W. M., Berkelbach van der Sprenkel, J. W., \& Dijkhuizen, R. M. (2010). Correspondence between altered functional and structural connectivity in the contralesional sensorimotor cortex after unilateral stroke in rats: a combined resting-state functional MRI and manganese-enhanced MRI study. Journal of Cerebral Blood Flow and Metabolism, 30(10), 1707-1711.

van Wijk, B. C. M., Stam, C. J., \& Daffertshofer, A. (2010). Comparing brain networks of different size and connectivity density using graph theory. PLOS ONE, 5(10), e13701.

Verhelst, H., Giraldo, D., Vander Linden, C., Vingerhoets, G., Jeurissen, B., \& Caeyenberghs, K. (2019). Cognitive training in young patients with traumatic brain injury: A fixel-based analysis. Neurorehabilitation and Neural Repair, 33(10), 813-824.

Vertes, P. E., Alexander-Bloch, A. F., Gogtay, N., Giedd, J. N., Rapoport, J. L., \& Bullmore, E. T. (2012). Simple models of human brain functional networks. Proceedings of the National Academy of Sciences, 109(15), 5868-5873.

Villamar, M. F., Portilla, A., Fregni, F., \& Zafonte, R. (2012). Noninvasive brain stimulation to modulate neuroplasticity in traumatic brain injury. Neuromodulation, 15(4), 337.

Wang, H. C., \& Ma, Y. Bin. (2010). Experimental models of traumatic axonal injury. Journal of Clinical Neuroscience, 17(2), 157-162.

Wang, L., Li, Y., Metzak, P., He, Y., \& Woodward, T. S. (2010). Age-related changes in topological patterns of large-scale brain functional networks during memory encoding and recognition. Neurolmage, 50, 862-872.

Wang, L., Su, L., Shen, H., \& Hu, D. (2012). Decoding lifespan changes of the human brain using restingstate functional connectivity MRI. PLOS ONE, 7(8).

Wang, M. L., Yu, M. M., Yang, D. X., Liu, Y. L., Wei, X. E., \& Li, W. B. (2018). Longitudinal microstructural changes in traumatic brain injury in rats: A diffusional kurtosis imaging, histology, and behavior study. American Journal of Neuroradiology, 39(9), 1650-1656.

Wang, R., Benner, T., Sorensen, A. G., \& Wedeen, V. J. (2007). Diffusion Toolkit: A software package for diffusion imaging data processing and tractography. Proc. ISMRM, 15, 3720. 
Wang, W., Zhang, H., Lee, D. H., Yu, J., Cheng, T., Hong, M., Jiang, S., Fan, H., Huang, X., Zhou, J., \& Wang, J. (2017). Using functional and molecular MRI techniques to detect neuroinflammation and neuroprotection after traumatic brain injury. Brain, Behavior and Immunity, 64, 344-353.

Wang, Z., Dai, Z., Gong, G., Zhou, C., \& He, Y. (2015). Understanding structural-functional relationships in the human brain: a large-scale network perspective. Neuroscientist, 21(3), 290-305.

Ward, N. S., Brown, M. M., Thompson, A. J., \& Frackowiak, R. S. J. (2003). Neural correlates of motor recovery after stroke: A longitudinal fMRI study. Brain, 126(11), 2476-2496.

Watson, B. D., Dietrich, W. D., Busto, R., Wachtel, M. S., \& Ginsberg, M. D. (1985). Induction of reproducible brain infarction by photochemically initiated thrombosis. Annals of Neurology, 17(5), 497-504.

Watts, D. J., \& Strogatz, S. H. (1998). Collective dynamics of 'small-world' networks. Nature, 393(6684), 440-442.

Westlye, L. T., Walhovd, K. B., Dale, A. M., Bjørnerud, A., Due-Tønnessen, P., Engvig, A., Grydeland, H., Tamnes, C. K., Østby, Y., \& Fjell, A. M. (2010). Life-span changes of the human brain white matter: Diffusion tensor imaging (DTI) and volumetry. Cerebral Cortex, 20(9), 2055-2068.

White, B. R., Bauer, A. Q., Snyder, A. Z., Schlaggar, B. L., Lee, J.-M., \& Culver, J. P. (2011). Imaging of functional connectivity in the mouse brain. PLOS ONE, 6(1), e16322.

White, J. G., Southgate, E., Thomson, J. N., \& Brenner, S. (1986). The mind of a worm. Philosophical Transactions of the Royal Society of London. Series B, Biological Sciences, 314(1165), 1-340.

Winklewski, P. J. (2018). Understanding the physiopathology behind axial and radial diffusivity changes - What do we know? Frontiers in Neurology, 9(2), 92.

Wright, D. K., Johnston, L. A., Kershaw, J., Ordidge, R., O'Brien, T. J., \& Shultz, S. R. (2017). Changes in apparent fiber density and track-weighted imaging metrics in white matter following experimental traumatic brain injury. Journal of Neurotrauma, 34(13), 2109-2118.

Wright, D. K., Trezise, J., Kamnaksh, A., Bekdash, R., Johnston, L. A., Ordidge, R., Semple, B. D., Gardner, A. J., Stanwell, P., O'Brien, T. J., Agoston, D. V., \& Shultz, S. R. (2016). Behavioral, blood, and magnetic resonance imaging biomarkers of experimental mild traumatic brain injury. Scientific Reports, 6(2), 1-16.

Wu, K., Taki, Y., Sato, K., Kinomura, S., Goto, R., Okada, K., Kawashima, R., He, Y., Evans, A. C., \& Fukuda, H. (2012). Age-related changes in topological organization of structural brain networks in healthy individuals. Human Brain Mapping, 33(3), 552-568.

Xiong, Y., Mahmood, A., \& Chopp, M. (2013). Animal models of traumatic brain injury. Nature Reviews Neuroscience, 14(2), 128-142.

Yendiki, A., Panneck, P., Srinivasan, P., Stevens, A., Zöllei, L., Augustinack, J., Wang, R., Salat, D., Ehrlich, S., Behrens, T., Jbabdi, S., Gollub, R., \& Fischl, B. (2011). Automated probabilistic reconstruction of white-matter pathways in health and disease using an atlas of the underlying anatomy. Frontiers in Neuroinformatics, 5(10), 23. 
Yin, Y., \& Yasuda, K. (2006). Similarity coefficient methods applied to the cell formation problem: A taxonomy and review. International Journal of Production Economics, 101(2), 329-352.

Zaaimi, B., Edgley, S. A., Soteropoulos, D. S., \& Baker, S. N. (2012). Changes in descending motor pathway connectivity after corticospinal tract lesion in macaque monkey. Brain, 135(7), 2277-2289.

Zalesky, A., \& Fornito, A. (2009). A DTI-derived measure of cortico-cortical connectivity. IEEE Xplore, 28(7), 1023-1036.

Zalesky, A., Fornito, A., Cocchi, L., Gollo, L. L., van den Heuvel, M., \& Breakspear, M. (2016). Connectome sensitivity or specificity: which is more important? Neurolmage, 142, 407-420.

Zeiler, S. R., \& Krakauer, J. W. (2013). The interaction between training and plasticity in the poststroke brain. Current Opinion in Neurology, 26(6), 609-616.

Zhang, H., Schneider, T., Wheeler-Kingshott, C. A., \& Alexander, D. C. (2012). NODDI: Practical in vivo neurite orientation dispersion and density imaging of the human brain. Neurolmage, 61, 1000-1016.

Zhang, J., Zhang, Y., Wang, L., Sang, L., Yang, J., Yan, R., Li, P., Wang, J., \& Qiu, M. (2017). Disrupted structural and functional connectivity networks in ischemic stroke patients. Neuroscience, 364, 212225.

Zhang, W., Yang, S., Li, C., Chen, L., \& Tang, Y. (2009). Stereological investigation of age-related changes of myelinated fibers in the rat cortex. Journal of Neuroscience Research, 87(13), 2872-2880.

Zhang, Z., Liao, W., Chen, H., Mantini, D., Ding, J. R., Xu, Q., Wang, Z., Yuan, C., Chen, G., Jiao, Q., \& Lu, G. (2011). Altered functional-structural coupling of large-scale brain networks in idiopathic generalized epilepsy. Brain, 134(10), 2912-2928.

Zhao, T., Cao, M., Niu, H., Zuo, X. N., Evans, A., He, Y., Dong, Q., \& Shu, N. (2015). Age-related changes in the topological organization of the white matter structural connectome across the human lifespan. Human Brain Mapping, 36(10), 3777-3792.

Zhu, W., Wen, W., He, Y., Xia, A., Anstey, K. J., \& Sachdev, P. (2012). Changing topological patterns in normal aging using large-scale structural networks. Neurobiology of Aging, 33(5), 899-913.

Zhuo, J., Xu, S., Proctor, J. L., Mullins, R. J., Simon, J. Z., Fiskum, G., \& Gullapalli, R. P. (2012). Diffusion kurtosis as an in vivo imaging marker for reactive astrogliosis in traumatic brain injury. Neurolmage, 59, 467-477.

Zuo, X.-N., Kelly, C., Di Martino, A., Mennes, M., Margulies, D. S., Bangaru, S., Grzadzinski, R., Evans, A. C., Zang, Y., Castellanos, X., \& Milham, M. P. (2010). Growing together and growing apart: Regional and sex differences in the lifespan developmental trajectories of functional homotopy. The Journal of Neuroscience, 30(45), 15034-15043. 


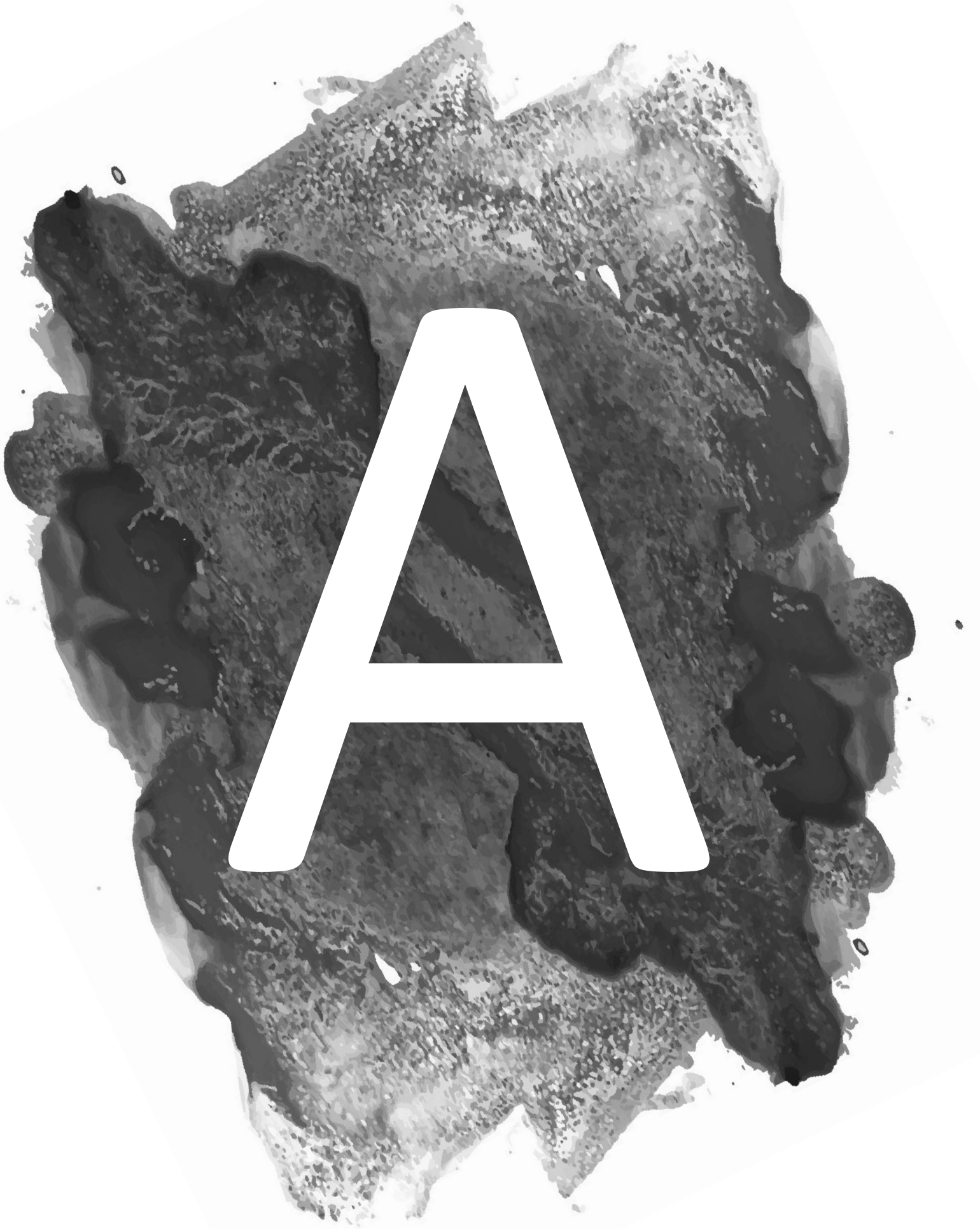


Addenda 



\section{SUMMARY}

The mammalian brain can be regarded as a highly complex and integrative network. Across the brain spatially distributed brain regions are structurally connected through axonal fiber bundles from micro- to macroscale. These structural connections allow continuous functional interaction between spatially distributed regions at different scales enabling mammalian brain function and behavior. As such, it is important to study the brain, as well as the consequences of brain disorders, from a network perspective in order to optimally understand pathological processes and recovery mechanisms. Magnetic resonance imaging (MRI) techniques offer exclusive means to perform longitudinal, multiparametric assessment of whole-brain structural and functional brain networks in health and diseases, such as after stroke or traumatic brain injury (TBI). This thesis describes MRI-based measurements of structural and functional connectivity in healthy brains (chapters 2, 3 and 5), during brain development (chapters 2 and 5), and after brain damage induced by lesion simulations (chapters 2 and 5), stroke (chapters 4 and 6) or TBI (chapter 7).

Chapter 1 elaborates on the network nature of the mammalian brain, measurements of structural and functional connectivity, network characterization by brain network analysis tools and how brain network characteristics are affected by ageing and pathology. Brain network science describes the brain as a collection of nodes (i.e. functional regions such as the primary motor cortex) and edges (i.e. the anatomical or functional connections between those regions), and offers tools to characterize structural and functional brain networks with different metrics, which provide information on its topological properties.

The healthy brain effectively combines global efficiency (i.e. integration) with local clustering (i.e. segregation). Deviation from this optimal balance between integration and segregation has been found in different brain pathologies and with ageing. For example, global efficiency shows an inverted- $U$ curve across the lifespan, with decreasing network density with ageing. These characteristics may be related to increased vulnerability of brain networks with ageing. After stroke and TBI, axonal connections in the white matter can be damaged, thereby altering functional interactions between brain regions. Due to the network nature of the brain, a focal lesion in a specific region or axonal connection can have global consequences, as connectivity with distant functional regions may be disturbed - a phenomenon called diaschisis. This may in turn alter brain function and behavior. Nevertheless, most patients show a certain degree of spontaneous recovery in the weeks and months following TBI or stroke, which may be related to brain plasticity mechanisms. Improved knowledge of the underlying mechanisms of brain damage and plasticity may ultimately lead to better diagnosis, prognosis and therapeutic strategies after acute brain injuries like stroke and TBI. Therefore the aim of this thesis was to characterize the spatiotemporal pattern of neuronal reorganization after brain damage due to stroke or TBI, as well as to determine the effect of ageing on brain network vulnerability to lesions. 
For this thesis several structural and functional MRI techniques were employed to assess structural and functional connectivity. Diffusion tensor imaging (DTI) was used to characterize white matter tissue, whereas diffusion-MRI based tractography was used to map large white matter tracts (i.e. structural connectivity). Resting-state functional MRI was used to measure the coherence in low-frequency blood oxygenation level-dependent (BOLD) signalling across brain regions (i.e. functional connectivity). Several network analyses approaches were used to characterize brain network topology, i.e. classical graph analysis, Bayesian exponential random graph models (ERGMs) and minimum spanning tree (MST) analysis. In addition, MRI findings in some chapters were related to behavioural performance and validated with histology.

Chapter 2 describes an assessment of the structural network topology across the human lifespan and how this topology is affected by lesion simulations in random and highly connected (hub) brain regions. Although descriptive network analyses have provided important insights into the organization of brain networks, these analyses have limitations for comparisons between networks that differ in size and density. Therefore Bayesian ERGMs were used, which may solve these issues by explaining the observed network topology by the joint contribution of underlying local network structures or features, for which we chose neurobiologically meaningful constructs such as connectedness, clustering, global efficiency and intrahemispheric connectivity. Structural networks were derived from whole-brain diffusion MRI-based tractography of 382 healthy adult subjects with an age range of 20 - 86 years. Networks were successfully generated from four local network structures, which resulted in excellent goodness-of-fit. The local structures - i.e. connectedness, local clustering, global efficiency and intrahemispheric connectivity - which give rise to the global network topology, were stable across the lifespan, contrary to descriptive network changes (e.g. decreased network density or global efficiency). Local network structures were robust against simulated random and (highly connected) hubnode damage, although the latter had a stronger effect in older people (above 70 years of age) as compared to the youngest age category. This may indicate that structural brain networks of older people are more vulnerable to hub-node damage. Furthermore, this chapter demonstrates the potential of Bayesian generative modeling to characterize the underlying network structures that drive the brain's global network topology at different developmental stages and/or under pathological conditions.

Chapter 3 reports on the validation of whole-brain structural connectome reconstructions in rats from recent diffusion-MRI based tractography approaches (constrained-spherical deconvolution (CSD)-based tractography and global tractography) against neuroanatomical connectivity from neuronal tracer studies. Although diffusionbased tractography offers unique abilities to map whole-brain structural connections in the mammalian brain, it measures white matter tracts indirectly, with suboptimal accuracy and 
reliability. Tractography results were evaluated across different anatomical sites and distances, and the influence of varying tractography parameter settings was examined. All tested tractography approaches, i.e. DTI-based, CSD-based and global tractography, generated considerable numbers of false positive and false negative connections. There was an overall trade-off between reconstruction sensitivity and specificity. The performance of all tractography approaches decreased with increasing spatial distance between connected regions. Similar patterns of trade-offs between sensitivity and specificity were found after applying streamline filtering, streamline thresholding and group-based average network thresholding. Despite the potential of CSD-based and global tractography to map complex fiber orientations (i.e. bending and crossing) at sub-voxel level, the connectome reconstruction accuracy, especially for long-distance connections, remains a challenge.

Chapter 4 describes the application of diffusion-based tractography to map structural connectivity in the contralesional hemisphere chronically after stroke in rats. Functional outcome after stroke depends on the local site of ischemic injury and on remote effects within connected networks, often extending into the contralesional hemisphere. In this chapter the structural network backbone topology was characterized with the MST approach, and network topologies of stroke and control animals were examined. The MST approach accounts for inter-subject and inter-group variations in network density, which allow unbiased characterization of network backbones that form the strongest connections in a network. Structural network backbones were found to be relatively stable across stroke and control animals. Several sensorimotor regions (e.g. upper lip and jaw regions of the primary somatosensory cortex) showed increased connection strength after stroke. Sensorimotor function correlated with specific contralesional sensorimotor network backbone measures of global integration and efficiency. Together, these findings point toward post-stroke adaptive reorganization of the contralesional sensorimotor network with recruitment of distinct sensorimotor regions, possibly through strengthening of connections, which may contribute to functional recovery.

Chapter 5 reports on the relationship between age-related brain network characteristics and stroke outcome. Diffusion MRI-based tractography and resting-state functional MRI were used to measure structural and functional brain connectivity. With a graph-based network approach, local and global network topology features were characterized and hub regions from structural and functional networks were identified in young adult (post-natal day 88) and aged rats (between post-natal days 760 and 880). Subsequently, three lesion simulations were performed based on the brain injury pattern in frequently applied rodent stroke models, i.e. a small cortical lesion, a subcortical lesion, or a large cortical plus subcortical lesion, for which the involved network regions were computationally removed. Global brain network features, such as global efficiency and local clustering, were stable between young adult and aged rats, but decreased after the simulation of a brain lesion. 
Local brain network features (such as hub-nodes) were different between young adult and aged rats, which also shifted differently after simulations of a brain lesion. This lesion simulation study demonstrates that age-dependent brain network status affects structural and functional network reorganization after stroke, particularly involving hub shifts, which may influence functional outcome. The local differences in structural and functional brain networks, including hub regions, between healthy young adult and aged rats, may have contributed to the different effects of lesion simulations between the two age groups. This underlines the critical role of age in the outcome of preclinical stroke studies in animal models, which often only involve young adult rodents, thereby limiting bench-to-bedside translation. Moreover, this chapter further shows that computational lesion studies offer a cheap and simple alternative to empirical studies and can complement or guide more complicated experimental studies in animal models and patients.

Chapter 6 describes how two important white matter tracts involved in sensorimotor function and interhemispheric communication - i.e. the corticospinal tract (CST) and corpus callosum - are spatiotemporally affected after experimental focal stroke, and how this is associated with functional connectivity and sensorimotor performance. Serial in vivo diffusion MRI, resting-state functional MRI and behavioural testing were conducted in twenty-five rats recovering from unilateral photothrombotic stroke in the sensorimotor cortex. White matter degeneration was found in the ipsilesional CST, indicated by lower fractional anisotropy and higher radial diffusivity. However, the integrity of the transcallosal tracts (i.e. interhemispheric structural connectivity) remained preserved. Functional connectivity between the ipsi- and contralesional sensorimotor cortices recovered after 28 weeks following initial loss of functional connectivity at one week and eight weeks poststroke. Recovery in functional connectivity paralleled the time-course of sensorimotor performance. This chapter shows that post-stroke motor recovery is possible, despite degeneration in the ipsilesional CST, and may rely on intact interhemispheric neuronal communication.

Chapter 7 reports on spatiotemporal patterns of structural and functional changes in grey and white matter regions from acute to chronic stages after mild and moderate TBI. $\mathrm{TBI}$ is a complex pathological process, which involves primary and secondary disease mechanisms. Despite clinical symptoms, a large majority of people with mild TBI has normal $\mathrm{CT}$ and MRI scans. As such present-day diagnostic imaging tools are insufficient to diagnose or classify low grades of TBI. Advanced neuroimaging techniques, such as diffusionweighted MRI and functional MRI, may yield novel biomarkers that may aid in the diagnosis of TBI. In this study fifty-two rats underwent serial functional resting-state MRI as well as structural ( $\mathrm{T}_{1^{-}}, \mathrm{T}_{2^{-}}$, and diffusion-weighted) MRI before and 1 hour, 1 day, 1 week, 1 month and 3-4 months after mild or moderate TBI induction. Early after moderate TBI, axial and radial diffusivities were increased, whereas fractional anisotropy was reduced, in the corpus 
callosum and bilateral hippocampi. These white matter diffusion parameters normalized over time, which was paralleled by similar patterns in $T_{2}$-weighted images as well as by patterns of loss and recovery in sensorimotor function. Histology revealed reduced myelin density in the acute stages after TBI, which was not detected in the chronic stages. The early occurrence of these diffusion changes and their transient nature (normalization within one week), is suggestive of the formation and subsequent resolution of white matter edema. This assumption is corroborated by the similar pattern in $T_{2}$ changes and the transient changes in myelin density. The absence of clear diffusion changes in response to microscopic histological alterations may suggest that contemporary diffusion MRI techniques lack sensitivity for detection of subtle white matter alterations. However, our multivariate logistic regression model analyses showed that combined assessment of different diffusion parameters can lead to improved differentiation between unaffected and mild or moderate TBI-affected brains acutely after TBI.

Chapter 8 summarizes and discusses the most important findings of this thesis, which are placed in broader scientific perspective. In addition, several pitfalls as well as potential future perspectives and directions are outlined. 



\section{SAMENVATTING}

Het brein van zoogdieren kan worden beschouwd als een zeer complex integratief netwerk. Over het gehele brein zijn verschillende functionele gebieden structureel met elkaar verbonden door middel van axonale bundels op micro- en macroniveau. Deze structurele verbindingen (connecties) maken continue functionele interactie mogelijk tussen verschillende spatieel verspreide hersengebieden op verschillende niveaus, wat leidt tot hersenfunctie en gedrag. Daarom is het van belang om het brein - alsook de gevolgen van aandoeningen in het brein - te bestuderen vanuit een netwerk perspectief, om zo pathologische processen en herstelmechanismen beter te kunnen begrijpen. 'Magnetic resonance imaging' (MRI) technieken bieden de unieke mogelijkheid om longitudinaal en multiparametrisch onderzoek te verrichten naar structurele en functionele hersennetwerken, in zowel het gezonde als het beschadigde brein, bijvoorbeeld na een beroerte of traumatisch hersenletsel ('traumatic brain injury', TBI). Dit proefschrift beschrijft MRI-gebaseerde metingen van structurele en functionele connectiviteit in het gezonde brein (hoofdstukken 2, 3 en 5), gedurende de ontwikkeling (hoofdstukken 2 en 5) en na hersenschade geïnduceerd door laesie simulaties (hoofdstukken 2 en 5), een beroerte (hoofdstukken 4 en 6) of TBI (hoofdstuk 7).

Hoofdstuk 1 weidt uit over het netwerkkarakter van het brein in zoogdieren, het meten van structurele en functionele connectiviteit, de karakterisatie van netwerken door netwerkanalyse technieken en hoe deze netwerk karakteristieken worden beïnvloed door veroudering en schade of pathologie in het brein. De netwerkwetenschap (specifiek gericht op het brein) beschrijft het brein als een verzameling knopen/knooppunten (de verschillende functionele hersengebieden, zoals de primaire motorische cortex) en lijnen (de anatomische of functionele verbindingen tussen deze gebieden). De netwerkwetenschap levert daarnaast methoden om structurele en functionele hersennetwerken te karakteriseren op basis van verschillende netwerkmaten die informatie verschaffen over topologische eigenschappen.

Het gezonde brein combineert op effectieve wijze globale efficiëntie (de integratie van informatie) met lokale clustering/specialisatie (de segregatie van informatie). Een afwijking van deze optimale balans tussen integratie en segregatie wordt gevonden in verschillende pathologieën van het brein alsook bij veroudering. Bijvoorbeeld, globale efficiëntie laat een omgekeerde- $U$ curve zien over het leven, met een afnemende netwerk dichtheid bij veroudering. Veranderingen in deze netwerk karakteristieken zijn mogelijk gerelateerd aan toegenomen kwetsbaarheid van netwerken bij veroudering. Na een beroerte of TBI kunnen axonale verbindingen in de witte stof beschadigen, waarbij functionele interacties tussen hersengebieden kunnen worden verstoord. Vanwege het netwerkkarakter van het brein kan een focale (plaatselijke) laesie in een specifiek gebied of axonale verbinding globale consequenties hebben, omdat verbindingen en interacties met verafgelegen 
hersengebieden kunnen worden verstoord - een fenomeen dat diaschisis wordt genoemd. Dit kan vervolgens weer leiden tot verstoorde hersenfunctie en gedragssymptomen. Desalniettemin laten de meeste patiënten een zekere mate van spontaan herstel zien in de weken en maanden na een beroerte of TBI, wat mogelijk gerelateerd is aan onderliggende plasticiteitsmechanismen. Verbeterde kennis van de onderliggende mechanismen van hersenschade en plasticiteit kan uiteindelijk leiden tot een betere diagnose, prognose en therapeutische behandeling bij acute hersenschade na beroerte of TBI. Daarom was het doel van dit proefschrift om de spatiotemporele patronen in neuronale reorganisatie na hersenschade (veroorzaakt door een beroerte of TBI) te karakteriseren, en om het effect van veroudering op de kwetsbaarheid van hersennetwerken voor laesies te bepalen.

Voor dit proefschrift werden verschillende structurele en functionele MRI technieken gebruikt om structurele en functionele connectiviteit te bepalen. Diffusion tensor imaging (DTI) is gebruikt om de witte stof te karakteriseren, terwijl diffusie MRI-gebaseerde tractografie is gebruikt om witte stof banen (de structurele connectiviteit) in kaart te brengen. Zogenaamde 'resting-state' functionele MRI is gebruikt om de coherentie in laagfrequente 'blood oxygenation level-dependent' (BOLD) signalen tussen verschillende gebieden (de functionele connectiviteit) te meten en in kaart te brengen. Verschillende netwerk analyse methoden zijn gebruikt om de topologie van hersennetwerken te karakteriseren, namelijk klassieke graaf analyse, Bayesiaanse 'exponential random graph models' (ERGMs) en de 'minimum spanning tree' (MST) methode. Aanvullend zijn de MRI bevindingen in sommige hoofdstukken gerelateerd aan gedragsmetingen en gevalideerd met histologie.

Hoofdstuk 2 beschrijft het in kaart brengen van de structurele netwerk topologie in verschillende stadia over het menselijke leven alsook hoe deze topologie wordt beïnvloed door het simuleren van laesies in willekeurige en sterk verbonden ('hub') gebieden. Hoewel beschrijvende netwerk analyses belangrijke inzichten hebben opgeleverd met betrekking tot de organisatie van hersennetwerken, hebben deze analyses ook intrinsieke beperkingen, met name voor vergelijkingen tussen netwerken van verschillende grootte en dichtheid. Om die reden is gebruik gemaakt van Bayesiaanse ERGMs, die mogelijk een oplossing bieden voor deze problemen door de geobserveerde globale netwerk topologie te verklaren door de gezamenlijke bijdrage van onderliggende lokale netwerkstructuren en -eigenschappen. Daarvoor gebruikten we neurobiologisch betekenisvolle constructen zoals mate van verbondenheid, clustering, globale efficiëntie en intrahemisferische connectiviteit. Structurele hersennetwerken werden gereconstrueerd met behulp van diffusie MRI-gebaseerde tractografie in 382 gezonde volwassenen in de leeftijd van 20 tot 86 jaar. Netwerken werden succesvol gegenereerd op basis van de vier locale netwerk structuren, wat resulteerde in een uitstekende 'goodness-of-fit'. De lokale structuren verbondenheid, lokale clustering, globale efficiëntie en intrahemisferische connectiviteit - 
die gezamenlijk het globale netwerk genereerden, waren stabiel over het leven, in tegenstelling tot beschrijvende netwerk veranderingen (zoals afgenomen netwerkdichtheid en globale efficiëntie). Lokale netwerkstructuren waren bestand (robuust) tegen gesimuleerde laesies in 'random' en (sterk verbonden) hub-gebieden, hoewel laatstgenoemde laesies een sterker effect hadden in oudere mensen (boven 70 jaar) in vergelijking met de jongste leeftijdscategorie. Dit kan betekenen dat structurele hersennetwerken in ouderen kwetsbaarder zijn voor hub-schade. Verder laat dit hoofdstuk de potentie zien van Bayesiaanse ERGMs om onderliggende netwerkstructuren te karakteriseren die bijdragen aan de globale netwerk topologie in verschillende fasen van het leven en/of onder pathologische condities.

Hoofdstuk 3 rapporteert over de validatie van structurele connectoom reconstructies van het rattenbrein met recent ontwikkelde diffusie MRI-gebaseerde tractografie methoden ('constrained spherical deconvolution' (CSD)-gebaseerde en globale tractografie) aan de hand van neuroanatomische connectiviteit uit neuronale tracer studies. Hoewel diffusie MRI-gebaseerde tractografie unieke mogelijkheden biedt om structurele verbindingen in het brein in kaart te brengen, bepaalt deze techniek witte stof banen op een indirecte manier met suboptimale accuratesse en betrouwbaarheid. De resultaten van tractografie werden onderzocht voor verschillende anatomische locaties en over verschillende afstanden. Ook de invloed van verschillende tractografieparameter instellingen werd onderzocht. Alle onderzochte tractografiemethoden (DTI-gebaseerde, CSD-gebaseerde en globale tractografie) genereerden een aanzienlijk aantal vals positieve en vals negatieve verbindingen. Er was sprake van een algemene 'trade-off tussen sensitiviteit en specificiteit van de reconstructies. De prestatie van de verschillende tractografiemethoden verminderde naarmate de afstanden tussen gebieden groter (en verbindingen langer) werden. Vergelijkbare patronen in 'trade-offs' tussen sensitiviteit en specificiteit werden waargenomen na het toepassen van 'streamline filtering', 'streamline thresholding' en het toepassen van gemiddelde groepsnetwerken en 'thresholding'. Ondanks de potentie van CSD-gebaseerde en globale tractografie om complexe axonale bundel oriëntaties (zoals gebogen en gekruiste bundels) in kaart te brengen op sub-voxel niveau, blijft de accuratesse van connectoom reconstructies, in het bijzonder voor lange afstand verbindingen, een uitdaging.

Hoofdstuk 4 beschrijft de toepassing van diffusie-gebaseerde tractografie om de structurele connectiviteit in kaart te brengen in de contralesionale hemisfeer chronisch na een beroerte in het rattenbrein. De functionele uitkomst na een beroerte is afhankelijk van de locatie van de ischemische schade, alsook van lange-afstand effecten door verbindingen en netwerken, zelfs tot in de contralesionale hemisfeer. In dit hoofdstuk werd de structurele netwerk 'backbone' topologie gekarakteriseerd met behulp van de MST methode, en werd de topologie van netwerken in ratten met een beroerte en controle ratten onderzocht. De 
MST methode houdt rekening met inter-subject en inter-groep variaties in de netwerkdichtheid. Dit zorgt voor een unbiased karakterisatie van netwerk 'backbones' die gevormd worden door de sterkste structurele verbindingen in een netwerk. Structurele netwerk 'backbones' waren relatief stabiel tussen dieren met of zonder een beroerte. Verschillende sensorimotorische gebieden (waaronder de lip- en kaakgebieden van de primaire somatosensorische cortex) vertoonden toegenomen structurele connectiviteit na een beroerte. De sensorimotorische functie correleerde met specifieke contralesionale sensorimotor netwerkmaten voor globale integratie en efficiëntie. Deze bevindingen wijzen op adaptieve reorganisatie van het contralesionale sensorimotorische netwerk na een beroerte, door middel van het rekruteren van andere sensorimotorische gebieden of het versterken van verbindingen, wat mogelijk bijdraagt aan functioneel herstel.

Hoofdstuk 5 gaat over de relatie tussen leeftijd gerelateerde netwerk karakteristieken in het brein en uitkomst na een beroerte. Diffusie MRI-gebaseerde tractografie en 'restingstate' functionele MRI werden gebruikt om structurele en functionele connectiviteit te meten. Met behulp van de klassieke netwerkbenadering werden lokale en globale karakteristieken van de netwerktopologie in kaart gebracht en werden (sterk verbonden) hub-gebieden bepaald in structurele en functionele netwerken van jong-volwassen (postnatale dag 88) en oude ratten (tussen post-natale dagen 760 en 880 ). Vervolgens werden drie laesiesimulaties uitgevoerd, gebaseerd op de schadepatronen in frequent toegepaste beroertemodellen in knaagdieren, te weten een kleine corticale laesie, een sub-corticale laesie, en een grote corticale plus sub-corticale laesie. Hierbij werden de betrokken netwerkgebieden computationeel verwijderd. Globale netwerkkarakteristieken, zoals globale efficiëntie en lokale clustering, waren stabiel tussen jongvolwassen en oude ratten, maar verminderden na simulatie van laesies in het brein. Lokale netwerkkarakteristieken (zoals hub-gebieden) waren verschillend tussen jongvolwassen en oude ratten. De verschuiving in hub-gebieden na laesies was ook anders in oude ratten dan in jongvolwassen ratten. Deze laesiesimulatie studie laat zien dat leeftijd gerelateerde netwerkkarakteristieken, en met name de veranderingen in hub-gebieden, invloed hebben op de structurele en functionele netwerkreorganisatie na een beroerte. Dit kan ook invloed hebben op de functionele uitkomst. De lokale verschillen in structurele en functionele netwerken, inclusief de verschillen in hub-gebieden, tussen jongvolwassen en oude ratten, hebben mogelijk bijgedragen aan de verschillende effecten van laesiesimulaties tussen de twee leeftijdsgroepen. Dit onderstreept de kritieke invloed van leeftijd op de uitkomst van preklinische studies naar beroerte, waarbij meestal gebruik wordt gemaakt van jongvolwassen knaagdieren, waardoor de ('bench-to-bedside') translatie mogelijk wordt beperkt. Dit hoofdstuk laat verder zien dat computationele studies een goedkoop en eenvoudig alternatief verschaffen voor empirische studies, en meer complexe experimentele studies in diermodellen en patiënten kunnen complementeren of sturen. 
Hoofdstuk 6 beschrijft onderzoek waarin werd bekeken hoe twee belangrijke witte stof banen, betrokken bij sensorimotorische functie en interhemisferische communicatie - de corticospinale tract (CST) en het corpus callosum - spatiotemporeel worden beïnvloed door een experimentele focale beroerte. Daarbij werd gekeken of dit is geassocieerd met veranderingen in functionele connectiviteit en gedragssymptomen. Hiervoor werden in vivo diffusie MRI, 'resting-state' functionele MRI en gedragstesten serieel uitgevoerd in ratten die herstellende waren van een unilaterale fototrombotische beroerte in de sensorimotorische cortex. Er werd witte stof degeneratie gevonden in de ipsilesionale CST, hetgeen werd aangegeven door lagere fractionele anisotropie (FA) en hogere radiale diffusiviteit (RD). Daarentegen was de integriteit van de transcallosale banen (interhemisferische structurele connectiviteit) niet aangetast. De functionele connectiviteit tussen de ipsi- en contralesionale sensorimotorische cortices was hersteld na 28 weken, na initieel verlies van functionele connectiviteit op één week en acht weken na de beroerte. Het herstel in functionele connectiviteit verliep parallel met het tijdsverloop van sensorimotorische functie. Dit hoofdstuk laat zien dat motorisch herstel na een beroerte mogelijk is, ondanks degeneratie van de ipsilesionale CST, en dat herstel mogelijk afhankelijk is van intacte interhemisferische neuronale communicatie.

Hoofdstuk 7 rapporteert over de spatiotemporele patronen in structurele en functionele veranderingen in grijze en witte stof gebieden, van de acute tot chronische fase na milde en matige TBI. TBI is een complex pathologisch proces, waarbij primaire en secundaire ziektemechanismen een rol spelen. Ondanks de klinische symptomen, heeft een meerderheid van milde TBI patiënten normale CT en MRI scans. Als zodanig zijn hedendaagse diagnostische imaging instrumenten onvoldoende voor de diagnose en classificatie van milde vormen van TBI. Geavanceerde neuroimaging technieken, zoals diffusie MRI en functionele MRI, leveren mogelijk nieuwe biomarkers op die bij kunnen dragen aan de diagnose van TBI. In deze studie ondergingen 52 ratten seriële 'resting-state' functionele MRI en structurele ( $T_{1^{-}}, T_{2}$ - en diffusie-gewogen) MRI vóór en op 1 uur, 1 dag, 1 week, 1 maand en 3-4 maanden na inductie van milde of matige TBI. Vroeg na matige TBI waren de axiale diffusiviteit $(A D)$ en radiale diffusiviteit (RD) toegenomen, terwijl de fractionele anisotropie (FA) was afgenomen, in het corpus callosum en de bilaterale hippocampi. Deze witte stof diffusie parameters normaliseerden over de tijd, hetgeen parallel verliep met het patroon dat gevonden werd in $T_{2}$-gewogen scans alsook met het patroon van verlies en herstel van sensorimotorische functie. Histologisch onderzoek liet vermindering van myeline dichtheid zien in de acute fase na $\mathrm{TBI}$, wat niet werd waargenomen in de chronische fase na TBI. De vroege veranderingen in diffusie parameters en de tijdelijke aard daarvan (normalisatie binnen een week) wijzen op de vorming en oplossing van witte stof oedeem. Deze aanname werd bevestigd door hetzelfde patroon in de $T_{2}$ veranderingen en de tijdelijke veranderingen in myeline dichtheid. De afwezigheid van 
duidelijke diffusie veranderingen na microscopische histologische aantasting, suggereert dat hedendaagse diffusie MRI technieken te weinig sensitiviteit hebben voor het detecteren van subtiele witte stof veranderingen. Echter, onze multivariate logistische regressie analyses lieten zien dat een gecombineerde analyse van verschillende diffusie parameters kan leiden tot verbeterde differentiatie tussen een gezond brein en een brein met acute milde of matige TBI.

Hoofdstuk 8 geeft een samenvatting van de belangrijkste bevindingen in dit proefschrift waarbij deze ook in breder wetenschappelijk perspectief worden geplaatst. Daarbij worden ook potentiële valkuilen en toekomstperspectieven besproken. 


\section{DANKWOORD}

Graag wil ik iedereen bedanken die heeft bijgedragen aan de totstandkoming van dit proefschrift. Enkele personen wil ik daarbij in het bijzonder bedanken.

Prof. dr. R.M. Dijkhuizen, beste Rick, hartelijk bedankt voor de goede begeleiding. Je gaf altijd uitgebreide en leerzame feedback op abstracts, presentaties en manuscripten. Ik heb bewondering voor de manier waarop je altijd de juiste aanpassingen en suggesties weet te doen, waardoor het geheel sterker wordt en daarmee ook beter overkomt. Ik heb daar veel van geleerd. Ook was het fijn om van gedachten te wisselen over de voortgang van een project, analyses of verkregen resultaten. Zelfs als iets tegenzit weet jij er toch nog wat positiefs van te maken en er in ieder geval uit te halen wat er in zit. Als je niet in overleg was stond je deur altijd (letterlijk) open. Hierdoor was het vrijwel altijd mogelijk om even van gedachten te wisselen als dat nodig was. Dat heb ik erg gewaardeerd. Ook je flexibele instelling in tijden dat het wegens privé omstandigheden lastig was om mijn werk goed uit te voeren heb ik erg gewaardeerd. Bedankt dat ik onderdeel mocht uitmaken van jouw onderzoeksgroep, ik kijk terug op een goede en leerzame periode.

Dr. W.M. Otte, beste Wim, jij ook hartelijk bedankt voor de goede begeleiding. Ik had geen betere dagelijkse begeleider kunnen treffen. Ik heb bewondering voor je creativiteit, vele originele ideeën en bovenal ook de effectiviteit en snelheid waarmee jij werkt. Hierdoor weet je een groot aantal projecten tegelijkertijd draaiende te houden. Bovendien ben je altijd bereikbaar, persoonlijk of digitaal, en reageer je doorgaans uitzonderlijk snel (en waar nodig uitgebreid) op ieder e-mailbericht. Ook je feedback op abstracts en manuscripten was altijd uitgebreid en leerzaam. Vanaf het begin heb je mij gestimuleerd om te leren scripten in verschillende programma's. Daarbij heb ik ook veel geleerd van jouw scripts en visualisaties (onder het motto 'beter goed gejat dan slecht verzonnen'). Tot op de dag van vadaag heb ik daar veel profijt van. Ik kijk ook met genoegen terug op de gezamenlijke reizen naar het buitenland voor congressen en - niet te vergeten - de reis naar Nigeria, een indrukwekkende reis die ook nog eens een mooie publicatie heeft opgeleverd. Verder hebben we redelijk vergelijkbare interesses, wat leidde tot vele gesprekken over wetenschap, filosofie, theologie en de uitwisseling van boeken en tijdschriften. Bedankt, ik heb het allemaal erg gewaardeerd.

De leden van de beoordelingscommissie, prof. dr. H.E. Hulshoff Pol, prof. dr. J.P.H. Burbach, prof. dr. G.J. Biessels, prof. dr. C.J. Stam en dr. ir. M. Froeling, wil ik bedanken voor het lezen en beoordelen van dit proefschrift. Daarbij wil ik ook prof. dr. F.E. Hoebeek en prof. dr. P.R. Bär (voorzitter) bedanken voor hun bereidheid deel te nemen aan de promotiecommissie. 
Graag wil ik al mijn co-auteurs bedanken voor de constructieve samenwerking en hun bijdragen aan dit proefschrift. I would like to thank all my co-authors for their constructive collaboration and contributions to this thesis.

Collega's van de Biomedical MR Imaging and Spectroscopy Group, Annette, Caroline, Gerard, Geralda, Erwin, Milou, Tessa, Julia, Jord, Eline, Bart, Anu, Vera, Esther en Maik, hartelijk bedankt voor de afgelopen jaren waarin ik onderdeel mocht zijn van de groep. Bedankt voor de goede samenwerking en ondersteuning, in welke vorm dan ook, waarmee jullie hebben bijgedragen aan de totstandkoming van dit proefschrift. Daarbij wil ik ook Marjan, Jacqueline en Maria bedanken voor alle secretariële ondersteuning. Ik kijk terug op een fijne periode waarin er naast werk ook tijd was voor ontspanning en goede gesprekken, tijdens koffiemomenten, borrels, uitjes, congressen en cursussen. Bedankt daarvoor.

Studenten die ik (samen met anderen) mocht begeleiden, Trude, Willem, Nikki en Christel, bedankt voor jullie inzet en fijne samenwerking. Het heeft een paar mooie afstudeerscripties opgeleverd die (deels) zijn verwerkt tot, of geresulteerd hebben in, een wetenschappelijk artikel.

Ouders, familie en vrienden, bedankt voor de vele fijne en ontspannende momenten. Bedankt ook voor de ondersteuning en hulp tijdens drukke of moeilijke periodes, door middel van praktische hulp, opvang van de kinderen, vervoer, ontspannig, gesprekken of op welke wijze dan ook. Het heeft mij (en ons) erg geholpen en heeft in die zin ook zeker bijgedragen aan het goed kunnen uitvoeren van mijn werkzaamheden en de totstandkoming van dit proefschrift.

Lieve Lianne, bedankt voor alles. Bedankt voor wie je bent, voor mij en voor de kinderen. Ik heb bewondering voor hoe je alles regelt bij ons thuis, alles is altijd goed georganiseerd. Als ik weer eens laat thuis kwam, of als ik een paar dagen (of weken) van huis was, dan regelde jij alles alleen. Dat waardeer ik enorm. Gelukkig hebben we ook veel mooie momenten beleefd, samen met onze kinderen. Lieve Julian, Rosalynn, Roan en Thymen, bedankt voor alle fijne momenten samen. We hebben het als gezin goed met elkaar, daar ben ik dankbaar voor. Ik hou van jullie. 


\section{LIST OF PUBLICATIONS}

\section{This Thesis}

M.R.T. Sinke, R.M. Dijkhuizen, A. Caimo, C.J. Stam \& W.M. Otte. Bayesian Exponential Random Graph Modelling of Whole-Brain Structural Networks across Lifespan. Neurolmage (2016), Vol. 135, 79-91.

M.R.T. Sinke, W.M. Otte, D. Christiaens, O. Schmitt, A. Leemans, A. van der Toorn, R.A. Sarabdjitsingh, M. Joëls \& R.M. Dijkhuizen. Diffusion MRI-Based Cortical Connectome Reconstruction - Dependency on Tractography Procedures and Neuroanatomical Characteristics. Brain Structure and Function (2018), Vol. 223 (5), 2269-2285.

M.R.T. Sinke, W.M. Otte, M.P.A. van Meer, A. van der Toorn \& R.M. Dijkhuizen. Modified Structural Network Backbone in the Contralesional Hemisphere Chronically after Stroke in Rat Brain. Journal of Cerebral Blood Flow \& Metabolism (2018), Vol. 38 (9), 1642-1653.

M. Straathof ${ }^{a}$, M.R.T. Sinke ${ }^{\text {a }}$, A. van der Toorn, P.L. Weerheim, W.M. Otte \& R.M. Dijkhuizen. Differences in Structural and Functional Networks between Young Adult and Aged Rat Brains before and after Stroke Lesion Simulations. Neurobiology of Disease (2019), Vol. 126, 23-25.

M.R.T. Sinke, G.A.F. van Tilborg, A.E. Meerwaldt, C.L. van Heijningen, A. van der Toorn, F. Rakib, M.H.M. Ali, K. Al-Saad, W.M. Otte \& R.M. Dijkhuizen. Remote Corticospinal Tract Degeneration after Cortical Stroke in Rats May Not Preclude Spontaneous Sensorimotor Recovery. Submitted.

M.R.T. Sinke, W.M. Otte, A.E. Meerwaldt, A.A. Franx, M.H.M. Ali, F. Rakib, A. van der Toorn, C.L. van Heijningen, C.E. Smeele, T. Ahmed, E.L.A. Blezer \& R.M. Dijkhuizen. Imaging Markers for the Characterization of Gray and White Matter Changes from Acute to Chronic Stages after Experimental Traumatic Brain Injury. Submitted. 


\section{Neuroscience}

G. Slinger, M.R.T. Sinke, K.P.J. Braun \& W.M. Otte. White Matter Abnormalities at a Regional and Voxel Level in Focal and Generalized Epilepsy: A Systematic Review and Meta-Analysis. Neurolmage Clinical (2015), Vol. 12, 902-909.

M. Straathof, M.R.T. Sinke, R.M. Dijkhuizen \& W.M. Otte. A Systematic Review on the Quantitative Relationship between Structural and Functional Network Connectivity Strength in Mammalian Brains. Journal of Cerebral Blood Flow \& Metabolism (2019), Vol. 39 (2), 189-209.

M.R.T. Sinke, J.W. Buitenhuis, F. van der Maas, J. Nwiboko, R.M. Dijkhuizen, E. van Diessen

${ }^{b}$ \& W.M. Otte ${ }^{b}$. The Power of Language: Functional Brain Network Topology of Deaf and Hearing in Relation to Sign Language Experience. Hearing Research (2019), Vol. 373, 32-47.

M. Straathof, M.R.T. Sinke, T.J.M. Roelofs, E.L.A. Blezer, R.A. Sarabdjitsingh, A. van der Toorn, O. Schmitt, W.M. Otte \& R.M. Dijkhuizen. Distinct Structure-Function Relationships across Cortical Regions and Connectivity Scales in the Rat Brain. Scientific Reports (2020), Vol. 10 (1), 1-14.

\section{Methodological}

V.T. van Hees, E. van Diessen, M.R.T. Sinke, J.W. Buitenhuis, F. van der Maas, L. Ridder \& W.M. Otte. Reliable and Automatic Epilepsy Classification with Affordable, Consumer-Grade Electroencephalography in Rural Sub-Saharan Africa. BioRxiv (2018), 324954.

H.J. Lamberink, W.M. Otte, M.R.T. Sinke, D. Lakens, P.P. Glasziou, J.K. Tijdink \& C.H. Vinkers. Statistical Power of Clinical Trials Increased While Effect Size Remained Stable: An Empirical Analysis of 136,212 Clinical Trials between 1975 and 2014. Journal of Clinical Epidemiology (2018), Vol. 102, 123-128.

M. Bot, C.M. Middeldorp, E.J.C. de Geus, H.M. Lau, M.R.T. Sinke, B. van Nieuwenhuizen, J.H. Smit, D.I. Boomsma \& B.W.J.H. Penninx. Validity of LIDAS (LIfetime Depression Assessment Self-report): A Self-Report Online Assessment of Lifetime Major Depressive Disorder. Psychologial Medicine (2017), Vol. 47 (2), 279-289.

\section{${ }^{b}$ Shared last author}




\section{CURRICULUM VITAE}

Michel Rogier Theodoor Sinke was born on the 27th of October 1986 in Gouda, the Netherlands. In 2009 he started the Bachelor (Biological) Psychology at the VU University, Amsterdam. After his graduation in 2012 he started the Master Neurosciences at VU University Amsterdam, part of the Neuroscience Campus Amsterdam. During this Research Master he performed scientific research as an intern at the Center for Childhood White Matter Disease at the VU Medical Center (VUmc) in Amsterdam (prof. dr. M.S. van der Knaap), as well as at the Leiden Institute for Brain and Cognition (LIBC) of the Leiden University Medical Center (LUMC) in Leiden (prof. dr. N.J.A. van der Wee). These internships were finished with two Master theses, by which he graduated Cum Laude in 2014. After graduation, Michel worked as a research assistant for one year at the department of Biological Psychology at the VU University in Amsterdam. From 2015 till 2019 he worked as a PhD candidate for the Biomedical MR Imaging and Spectroscopy Group at the Imaging department of the University Medical Center Utrecht (UMCU). Under supervision of prof. dr. R.M. Dijkhuizen and dr. W.M. Otte he performed research which finally resulted in this PhD thesis. Since September 2019 Michel is working for the programme Translational Research of ZonMw, The Netherlands Organisation for Health Research and Development. 




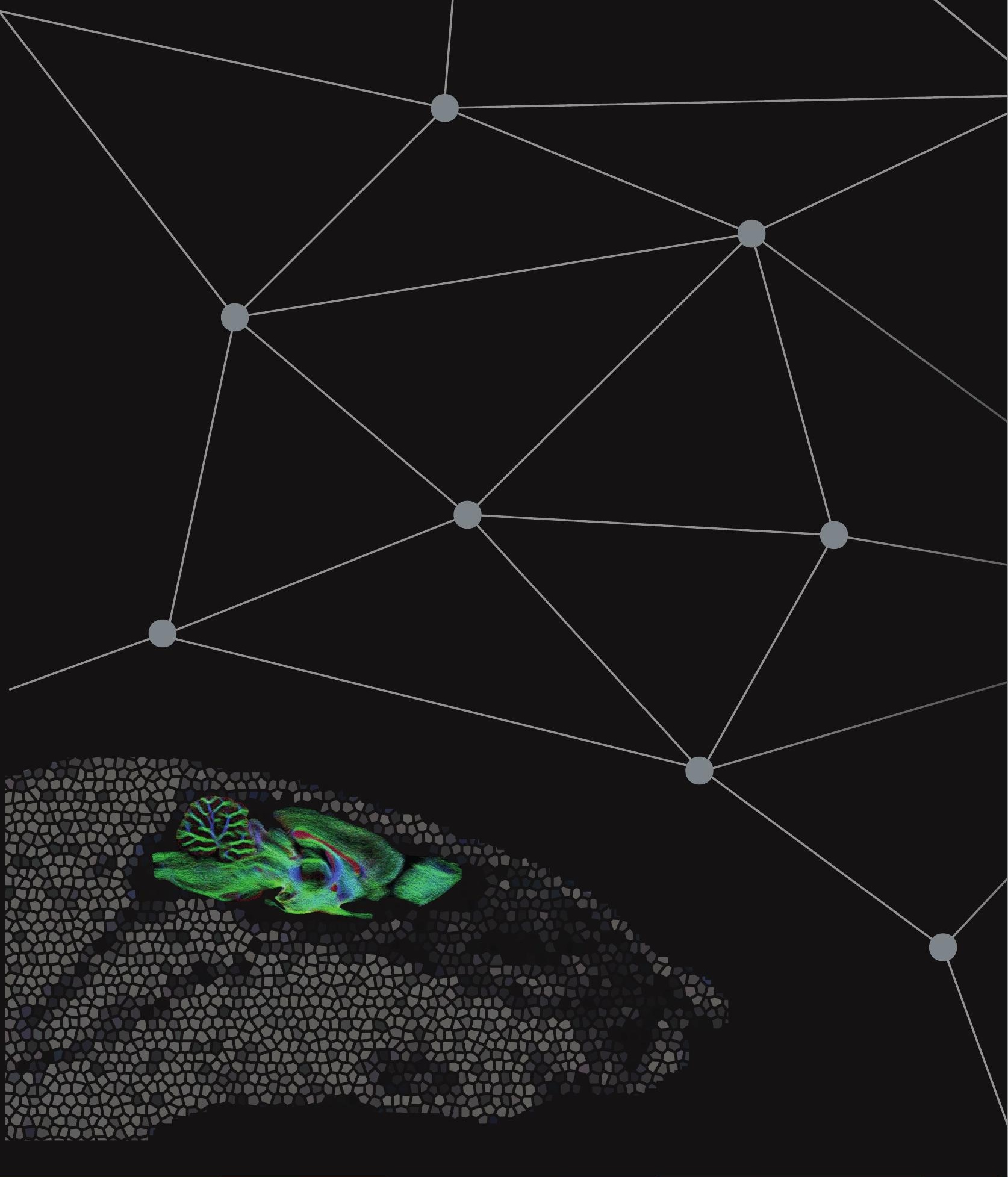

UMC Utrecht Universiteit Utrecht 